\title{
Die Neigung zu Schwarzfleckigkeit bei Kartoffeln (Solanum tuberosum) unter besonderer Berücksichtigung der Knollenphysiologie
}

\author{
Dissertation \\ zur Erlangung des Doktorgrades \\ der Fakultät für Agrarwissenschaften \\ der Georg-August-Universität Göttingen
}

vorgelegt von

Antje Wulkow

geboren in Schwedt/Oder

Göttingen, Mai 2009 
D 7

1. Referentin/Referent:

2. Koreferentin/Koreferent:

Tag der mündlichen Prüfung:
Prof. Dr. E. Pawelzik

Prof. Dr. B. Märländer

28. Mai 2009 


\section{Inhaltsverzeichnis}

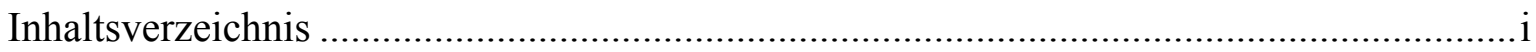

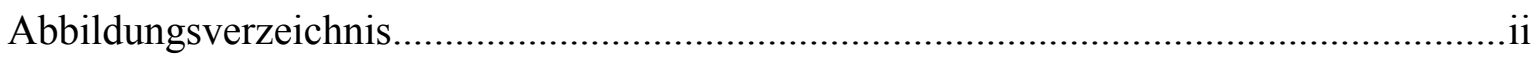

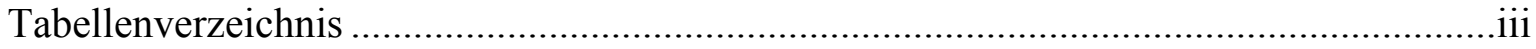

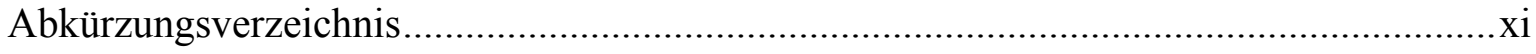

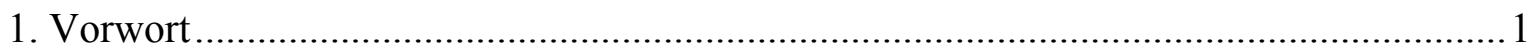

2. Blackspot susceptibility in comparison with the antioxidant activity of potato (Solanum

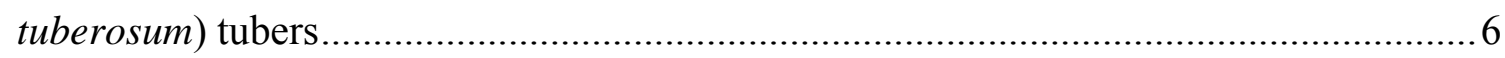

3. Water relations in potato (Solanum tuberosum) tubers in comparison to their blackspot

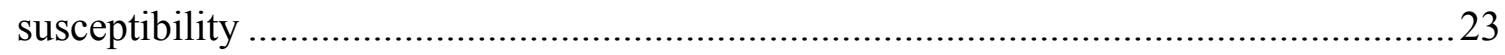

4. Nutrient concentrations in potato (Solanum tuberosum) tubers in comparison with their

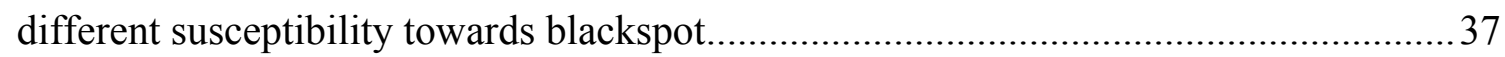

5. Blackspot bruise formation in potato (Solanum tuberosum) tubers in relation to the

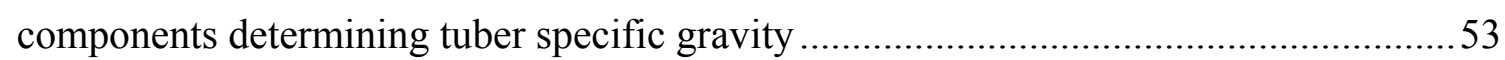

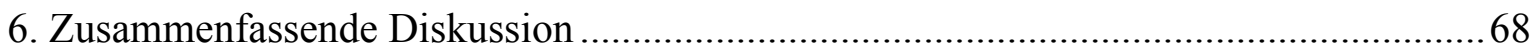

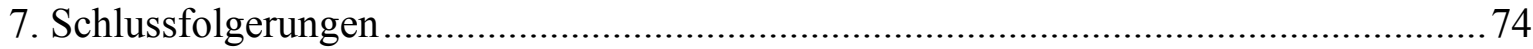

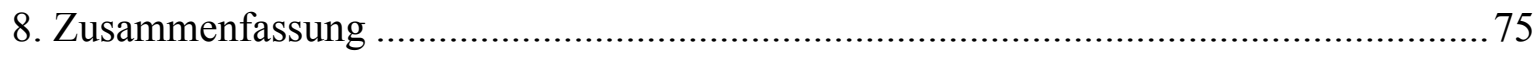

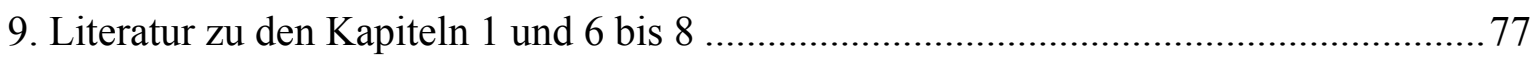

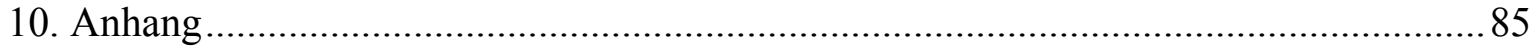

Danksagung

Lebenslauf 


\section{Abbildungsverzeichnis}

Abbildung 1.1 Knollen einer Kartoffelsorte (A) mit zunehmender Neigung zu Schwarzfleckigkeit (v.1.) nach der Ernte 2006

Abbildung 1.2 Knollen einer Kartoffelsorte (B) mit zunehmender Neigung zu Schwarzfleckigkeit (v.1.) nach der Ernte 2006

Figure 2.1 Blackspot index (BSI, \%) of potato tubers differing in their specific gravity $\left(\mathrm{kg} \mathrm{L}^{-1}\right)$ in comparison to their discolouration potential (DP, $\mathrm{AU}_{475 \mathrm{~nm}}$ ) and antioxidant activity (FRAP, mmol kg-1 FM) after harvest (I) in 2005, following five (II) and eight (III) months of storage, respectively and after harvest (IV) in 2006, following five (V) and eight (VI) months of storage, respectively, irrespective of cultivar, statistical evalutions are shown in Table 2.1

Figure 3.1 BSI (\%) of potato tubers of different specific gravities in comparison to their water concentration $\left(\mathrm{g} \mathrm{kg}^{-1} \mathrm{FM}\right)$, osmolality ( $\left.\mathrm{mmol} \mathrm{kg}^{-1}\right)$, osmotic potential $(\mathrm{MPa})$, water potential $(\mathrm{MPa})$, pressure potential $(\mathrm{MPa})$ and modulus of elasticity (MPa) after harvest (A) 2006, following five (B) and (C) eight months of storage, respectively, and after harvest (D) 2007, following five (E) and eight (F) months of storage, respectively, statistical evaluations are shown in Table 3.1 and 3.2

Figure 4.1 Relationship between tuber specific gravity $\left(\mathrm{kg} \mathrm{L}^{-1}\right)$ and BSI (\%) as well as tuber nutrient concentrations $\left(\mathrm{mg} \mathrm{kg}^{-1} \mathrm{FW}\right)$ and BSI (\%) determined in 2006 and 2007 after the pre-harvest, harvest and five and eight months of storage

Figure 5.1 Distribution (\%) of tubers differing in their specific gravity within a cultivar (A), blackspot index (BSI, \%) and tuber components attributed to specific gravity (B) of potato tubers harvested 2005 (I) and stored for five (II) and eight (III) months, respectively

Figure 5.2 Distribution (\%) of tubers differing in their specific gravity within a cultivar (A), blackspot index (BSI, \%) and tuber components attributed to specific gravity (B) of potato tubers harvested 2006 (I) and stored for five (II) and eight (III) months, respectively

Figure 5.3 Distribution (\%) of tubers differing in their specific gravity within a cultivar (A), blackspot index (BSI, \%) and tuber components attributed to specific gravity (B) of potato tubers harvested 2007 (I) and stored for five (II) and eight (III) months, respectively 


\section{Tabellenverzeichnis}

Table 2.1 Relationships between blackspot susceptibility (BSI, \%), discolouration potential (DP, $\mathrm{AU}_{475 \mathrm{~nm}}$ ) and antioxidant activity (FRAP, $\mathrm{mmol} \mathrm{kg}^{-1} \mathrm{FM}$ ) of tubers differing in their specific gravity $\left(\mathrm{kg} \mathrm{L}^{-1}\right)$ as Pearson Correlation Coefficient irrespective of cultivar, data refer to Figure 2.1

Table 2.2 Blackspot index (BSI, \%) of potato cultivars in comparison with their discolouration potential (DP, $\mathrm{AU}_{475 \mathrm{~nm}}$ ) and antioxidant activity (FRAP, mmol $\mathrm{kg}^{-1} \mathrm{FM}$ ) after harvest, five (5), and eight (8) months of storage of two vegetation periods (2005 and 2006) irrespective of their specific gravity

Table 2.3 Antioxidant activity (relative H-ORAC, mmol GAE kg-1 FM; FRAP, mmol kg-1 $\mathrm{FM}$ ) of tuber tissue of potato cultivars differing in their blackspot susceptibility (BSI, \%) harvested in 2005

Table 2.4 Antioxidant activity (relative H-ORAC, mmol GAE kg ${ }^{-1}$ FM; FRAP, mmol kg-1 $\mathrm{FM}$ ) of tuber tissue of potato cultivars differing in their blackspot susceptibility (BSI, \%) harvested in 2006

Table 3.1 Water concentration $\left(\mathrm{g} \mathrm{kg}^{-1} \mathrm{FM}\right)$, water potential (MPa), osmolality $\left(\mathrm{mmol} \mathrm{kg}{ }^{-1}\right)$, osmotic potential (MPa), modulus of elasticity (MPa), pressure potential $(\mathrm{MPa})$ and blackspot index $(\%)$ of tubers differing in their specific gravity $\left(\mathrm{kg} \mathrm{L}^{-1}\right)$ harvested 2006 and stored for five and eight months

Table 3.2 Water concentration ( $\left.\mathrm{g} \mathrm{kg}^{-1} \mathrm{FM}\right)$, water potential (MPa), osmolality $\left(\mathrm{mmol} \mathrm{kg}{ }^{-1}\right)$, osmotic potential $(\mathrm{MPa})$, modulus of elasticity $(\mathrm{MPa})$, pressure potential $(\mathrm{MPa})$ and blackspot index $(\%)$ of tubers differing in their specific gravity $\left(\mathrm{kg} \mathrm{L}^{-1}\right)$ harvested 2007 and stored for five and eight months

Table 4.1 The ratio of macro:micro nutrients of tubers harvested at different stages of haulm maturity in 2006 and 2007 and stored for five and eight months, respectively

Table 4.2 Concentration of selected nutrients $\left(\mathrm{mg} \mathrm{kg}^{-1} \mathrm{FW}\right)$ in potato tubers differing in their blackspot susceptibility irrespective of cultivar, vegetation period and time of storage in comparison to published data of these nutrients $\left(\mathrm{mg} \mathrm{kg}^{-1} \mathrm{FW}\right)$ in potato tubers irrespective of cultivar, vegetation period and time of storage 
Table 4.3 Relative concentration (\%) of selected nutrients in potato tubers differing in their blackspot susceptibility irrespective of cultivar, vegetation period and time of storage in comparison to the published data of relative concentration $(\%)$ of these nutrients in potato tubers irrespective of cultivar, vegetation period and time of storage

Tabelle 6.1 Die Wirkung nicht-enzymatischer Antioxidantien in Kartoffeln und anderen pflanzlichen Produkten ermittelt unter Anwendung verschiedener Analysemethoden

Tabelle 10.1 Schwarzfleckigkeit [Mittelwert $(\bar{x})$ und Standartabweichung $\left(\sigma_{\mathrm{x}}\right)$ ] $(\%)$ in Sorten und Knollen verschiedener spezifischer Dichten $\left(\mathrm{kg} \mathrm{L}^{-1}\right)$ nach der Ernte 2005 und nach fünf und acht Monaten Lagerung 86

Tabelle 10.2 Schwarzfleckigkeit [Mittelwert $(\bar{x})$ und Standartabweichung $\left.\left(\sigma_{\mathrm{x}}\right)\right](\%)$ in Sorten und Knollen verschiedener spezifischer Dichten $\left(\mathrm{kg} \mathrm{L}^{-1}\right)$ nach der Vorernte und Ernte 2006 und nach fünf und acht Monaten Lagerung

Tabelle 10.3 Schwarzfleckigkeit [Mittelwert $(\bar{x})$ und Standartabweichung $\left(\sigma_{\mathrm{x}}\right)$ ] $(\%)$ in Sorten und Knollen verschiedener spezifischer Dichten $\left(\mathrm{kg} \mathrm{L}^{-1}\right)$ nach der Vorernte und Ernte 2007 und nach fünf und acht Monaten Lagerung $\quad 88$

Tabelle 10.4 Schwarzfleckigkeit [Mittelwert $(\bar{x})$ und Standartabweichung $\left(\sigma_{\mathrm{x}}\right)$ ] $(\%)$ nach teilweiser Vorernte und Ernte in 2005, 2006 und 2007 und nach fünf und acht Monaten Lagerung von Sorten, deren Knollen nicht nach spezifischen Dichten getrennt wurden aber teilweise bei $4^{\circ} \mathrm{C}$ und $8^{\circ} \mathrm{C}$ gelagert worden sind

Tabelle 10.5 Oxidatives Potential [Mittelwert $(\bar{x})$ und Standartabweichung $\left(\sigma_{\mathrm{x}}\right)$ ] $\left(\mathrm{AU}_{475 \mathrm{~nm}}\right)$ von Sorten und Knollen verschiedener spezifischer Dichten $\left(\mathrm{kg} \mathrm{L}^{-1}\right)$ nach der Ernte 2005 und nach fünf und acht Monaten Lagerung

Tabelle 10.6 Oxidatives Potential [Mittelwert $(\bar{x})$ und Standartabweichung $\left(\sigma_{\mathrm{x}}\right)$ ] $\left(\mathrm{AU}_{475 \mathrm{~nm}}\right)$ von Sorten und Knollen verschiedener spezifischer Dichten $\left(\mathrm{kg} \mathrm{L}^{-1}\right)$ nach der Ernte 2006 und nach fünf und acht Monaten Lagerung

Tabelle 10.7 Antioxidative Kapazität (FRAP) [Mittelwert $(\bar{x})$ und Standartabweichung $\left.\left(\sigma_{\mathrm{x}}\right)\right]\left(\mathrm{mmol} \mathrm{kg}{ }^{-1} \mathrm{FM}\right)$ von Sorten und Knollen verschiedener spezifischer Dichten $\left(\mathrm{kg} \mathrm{L}^{-1}\right)$ nach der Ernte 2005 und nach fünf und acht Monaten Lagerung 
Tabelle 10.8 Antioxidative Kapazität (FRAP) [Mittelwert $(\bar{x})$ und Standartabweichung $\left.\left(\sigma_{\mathrm{x}}\right)\right]\left(\mathrm{mmol} \mathrm{kg}{ }^{-1} \mathrm{FM}\right)$ von Sorten und Knollen verschiedener spezifischer Dichten $\left(\mathrm{kg} \mathrm{L}^{-1}\right)$ nach der Ernte 2006 und nach fünf und acht Monaten Lagerung

Tabelle 10.9 Antioxidative Kapazität (FRAP) [Mittelwert $(\bar{x})$ und Standartabweichung $\left.\left(\sigma_{\mathrm{x}}\right)\right]\left(\mathrm{mmol} \mathrm{kg}{ }^{-1} \mathrm{FM}\right)$ von Schalen- und Markgewebe nach der Ernte der Sorten in 2005 und 2006, die Knollen wurden nicht nach spezifischen Dichten getrennt

Tabelle 10.10 Antioxidative Kapazität (H-ORAC) [Mittelwert $(\bar{x})$ und Standartabweichung $\left(\sigma_{\mathrm{x}}\right)$ ] (mmol GAE $\mathrm{kg}^{-1} \mathrm{FM}$ ) von Schalen- und Markgewebe nach der Ernte der Sorten in 2005 und 2006, die Knollen wurden nicht nach spezifischen Dichten getrennt

Tabelle 10.11 Wasserkonzentration [Mittelwert $(\bar{x})$ und Standartabweichung $\left(\sigma_{\mathrm{x}}\right)$ ] $\left(\mathrm{g} \mathrm{kg}^{-1} \mathrm{FM}\right)$ in Sorten und Knollen verschiedener spezifischer Dichten $\left(\mathrm{kg} \mathrm{L}^{-1}\right)$ nach der Ernte 2006 und nach fünf und acht Monaten Lagerung

Tabelle 10.12 Wasserkonzentration [Mittelwert $(\bar{x})$ und Standartabweichung $\left(\sigma_{\mathrm{x}}\right)$ ] $\left(\mathrm{g} \mathrm{kg}^{-1} \mathrm{FM}\right)$ in Sorten und Knollen verschiedener spezifischer Dichten $\left(\mathrm{kg} \mathrm{L}^{-1}\right)$ nach der Ernte 2007 und nach fünf und acht Monaten Lagerung

Tabelle 10.13 Wasserkonzentration [Mittelwert $(\bar{x})$ und Standartabweichung $\left(\sigma_{\mathrm{x}}\right)$ ] ( $\mathrm{g} \mathrm{kg}^{-1}$ FM) in Kronen- und Nabelgewebe nach der Ernte 2006 und 2007 und nach fünf und acht Monaten Lagerung der Sorten, deren Knollen nicht nach spezifischen Dichten getrennt wurden

Tabelle 10.14 Wasserpotential [Mittelwert $(\bar{x})$ und Standartabweichung $\left(\sigma_{\mathrm{x}}\right)$ ] $(\mathrm{MPa})$ in Sorten und Knollen verschiedener spezifischer Dichten $\left(\mathrm{kg} \mathrm{L}^{-1}\right)$ nach der Ernte 2006 und nach fünf und acht Monaten Lagerung

Tabelle 10.15 Wasserpotential [Mittelwert $(\bar{x})$ und Standartabweichung $\left(\sigma_{\mathrm{x}}\right)$ ] $(\mathrm{MPa})$ in Sorten und Knollen verschiedener spezifischer Dichten $\left(\mathrm{kg} \mathrm{L}^{-1}\right)$ nach der Ernte 2007 und nach fünf und acht Monaten Lagerung

Tabelle 10.16 Wasserpotential [Mittelwert $(\bar{x})$ und Standartabweichung $\left(\sigma_{\mathrm{x}}\right)$ ] (MPa) in Kronen- und Nabelgewebe nach der Ernte 2006 und 2007 und nach fünf und acht Monaten Lagerung der Sorten, deren Knollen nicht nach spezifischen Dichten getrennt wurden 
Tabelle 10.17 Osmolalität [Mittelwert $(\bar{x})$ und Standartabweichung $\left.\left(\sigma_{\mathrm{x}}\right)\right]\left(\mathrm{mmol} \mathrm{kg}^{-1}\right)$ in Sorten und Knollen verschiedener spezifischer Dichten $\left(\mathrm{kg} \mathrm{L}^{-1}\right)$ nach der Ernte 2006 und nach fünf und acht Monaten Lagerung

Tabelle 10.18 Osmolalität [Mittelwert $(\bar{x})$ und Standartabweichung $\left.\left(\sigma_{\mathrm{x}}\right)\right]\left(\mathrm{mmol} \mathrm{kg}^{-1}\right)$ in Sorten und Knollen verschiedener spezifischer Dichten $\left(\mathrm{kg} \mathrm{L}^{-1}\right)$ nach der Ernte 2007 und nach fünf und acht Monaten Lagerung 102

Tabelle 10.19 Osmolalität [Mittelwert $\left({ }^{\bar{X}}\right)$ und Standartabweichung $\left.\left(\sigma_{\mathrm{x}}\right)\right]\left(\mathrm{mmol} \mathrm{kg}^{-1}\right)$ in Kronen- und Nabelgewebe nach der Ernte 2006 und 2007 und nach fünf und acht Monaten Lagerung der Sorten, deren Knollen nicht nach spezifischen Dichten getrennt wurden

Tabelle 10.20 Osmotisches Potential [Mittelwert $(\bar{x})$ und Standartabweichung $\left(\sigma_{\mathrm{x}}\right)$ ] $(\mathrm{MPa})$ in Sorten und Knollen verschiedener spezifischer Dichten $\left(\mathrm{kg} \mathrm{L}^{-1}\right)$ nach der Ernte 2006 und nach fünf und acht Monaten Lagerung 104

Tabelle 10.21 Osmotisches Potential [Mittelwert $(\bar{x})$ und Standartabweichung $\left(\sigma_{\mathrm{x}}\right)$ ] $(\mathrm{MPa})$ in Sorten und Knollen verschiedener spezifischer Dichten $\left(\mathrm{kg} \mathrm{L}^{-1}\right)$ nach der Ernte 2007 und nach fünf und acht Monaten Lagerung 105

Tabelle 10.22 Osmotisches Potential [Mittelwert $(\bar{X})$ und Standartabweichung $\left(\sigma_{\mathrm{x}}\right)$ ] (MPa) in Kronen- und Nabelgewebe nach der Ernte 2006 und 2007 und nach fünf und acht Monaten Lagerung der Sorten, deren Knollen nicht nach spezifischen Dichten getrennt wurden

Tabelle 10.23 Binnendruck (Turgor) [Mittelwert $(\bar{x})$ und Standartabweichung $\left(\sigma_{\mathrm{x}}\right)$ ] $(\mathrm{MPa})$ in Sorten und Knollen verschiedener spezifischer Dichten $\left(\mathrm{kg} \mathrm{L}^{-1}\right)$ nach der Ernte 2006 und nach fünf und acht Monaten Lagerung

Tabelle 10.24 Binnendruck (Turgor) [Mittelwert $(\bar{x})$ und Standartabweichung $\left(\sigma_{\mathrm{x}}\right)$ ] $(\mathrm{MPa})$ in Sorten und Knollen verschiedener spezifischer Dichten $\left(\mathrm{kg} \mathrm{L}^{-1}\right)$ nach der Ernte 2007 und nach fünf und acht Monaten Lagerung 108

Tabelle 10.25 Binnendruck (Turgor) [Mittelwert $(\bar{x})$ und Standartabweichung $\left(\sigma_{\mathrm{x}}\right)$ ] (MPa) in Kronen- und Nabelgewebe nach der Ernte 2006 und 2007 und nach fünf und acht Monaten Lagerung der Sorten, deren Knollen nicht nach spezifischen Dichten getrennt wurden

Tabelle 10.26 Volumetrischer Modulus der Elastizität [Mittelwert $(\bar{x})$ und Standartabweichung $\left.\left(\sigma_{\mathrm{x}}\right)\right](\mathrm{MPa})$ in Sorten und Knollen verschiedener spezifischer Dichten $\left(\mathrm{kg} \mathrm{L}^{-1}\right)$ nach der Ernte 2006 und nach fünf und acht Monaten Lagerung 
Tabelle 10.27 Volumetrischer Modulus der Elastizität [Mittelwert $\left({ }^{\bar{X}}\right)$ und Standartabweichung $\left(\sigma_{\mathrm{x}}\right)$ ] $(\mathrm{MPa})$ in Sorten und Knollen verschiedener spezifischer Dichten $\left(\mathrm{kg} \mathrm{L}^{-1}\right)$ nach der Ernte 2007 und nach fünf und acht Monaten Lagerung

Tabelle 10.28 Volumetrischer Modulus der Elastizität [Mittelwert $(\bar{X})$ und Standartabweichung $\left(\sigma_{\mathrm{x}}\right)$ ] $(\mathrm{MPa})$ in Kronen- und Nabelgewebe nach der Ernte 2006 und 2007 und nach fünf und acht Monaten Lagerung der Sorten, deren Knollen nicht nach spezifischen Dichten getrennt wurden

Tabelle 10.29 Borkonzentration [Einzelwerte] ( $\left.\mathrm{mg} \mathrm{kg}^{-1} \mathrm{FM}\right)$ in Sorten und Knollen verschiedener spezifischer Dichten $\left(\mathrm{kg} \mathrm{L}^{-1}\right)$ nach der Vorernte und Ernte 2006 und nach fünf und acht Monaten Lagerung

Tabelle 10.30 Borkonzentration [Einzelwerte] ( $\left.\mathrm{mg} \mathrm{kg}^{-1} \mathrm{FM}\right)$ in Sorten und Knollen verschiedener spezifischer Dichten $\left(\mathrm{kg} \mathrm{L}^{-1}\right)$ nach der Vorernte und Ernte 2007 und nach fünf und acht Monaten Lagerung

Tabelle 10.31 Eisenkonzentration [Einzelwerte] $\left(\mathrm{mg} \mathrm{kg}^{-1} \mathrm{FM}\right)$ in Sorten und Knollen verschiedener spezifischer Dichten $\left(\mathrm{kg} \mathrm{L}^{-1}\right)$ nach der Vorernte und Ernte 2006 und nach fünf und acht Monaten Lagerung

115

Tabelle 10.32 Eisenkonzentration [Einzelwerte] $\left(\mathrm{mg} \mathrm{kg}^{-1} \mathrm{FM}\right)$ in Sorten und Knollen verschiedener spezifischer Dichten $\left(\mathrm{kg} \mathrm{L}^{-1}\right)$ nach der Vorernte und Ernte 2007 und nach fünf und acht Monaten Lagerung

Tabelle 10.33 Selenkonzentration [Einzelwerte] $\left(\mathrm{mg} \mathrm{kg}^{-1} \mathrm{FM}\right)$ in Sorten und Knollen verschiedener spezifischer Dichten $\left(\mathrm{kg} \mathrm{L}^{-1}\right)$ nach der Vorernte und Ernte 2006 und nach fünf und acht Monaten Lagerung

Tabelle 10.34 Selenkonzentration [Einzelwerte] ( $\left.\mathrm{mg} \mathrm{kg}^{-1} \mathrm{FM}\right)$ in Sorten und Knollen verschiedner spezifischer Dichten $\left(\mathrm{kg} \mathrm{L}^{-1}\right)$ nach der Vorernte und Ernte 2007 und nach fünf und acht Monaten Lagerung

Tabelle 10.35 Kalziumkonzentration [Einzelwerte] ( $\left.\mathrm{mg} \mathrm{kg}^{-1} \mathrm{FM}\right)$ in Sorten und Knollen verschiedener spezifischer Dichten $\left(\mathrm{kg} \mathrm{L}^{-1}\right)$ nach der Vorernte und Ernte 2006 und nach fünf und acht Monaten Lagerung

Tabelle 10.36 Kalziumkonzentration [Einzelwert] (mg kg-1 FM) in Sorten und Knollen verschiedener spezifischer Dichten $\left(\mathrm{kg} \mathrm{L}^{-1}\right)$ nach der Vorernte und Ernte 2007 und nach fünf und acht Monaten Lagerung 120

Tabelle 10.37 Magnesiumkonzentration [Einzelwerte] ( $\mathrm{mg} \mathrm{kg}^{-1} \mathrm{FM}$ ) in Sorten und Knollen verschiedener spezifischer Dichten $\left(\mathrm{kg} \mathrm{L}^{-1}\right)$ nach der Vorernte und Ernte 2006 und nach fünf und acht Monaten Lagerung 
Tabelle 10.38 Magnesiumkonzentration [Einzelwerte] ( $\mathrm{mg} \mathrm{kg}^{-1} \mathrm{FM}$ ) in Sorten und Knollen verschiedener spezifischer Dichten $\left(\mathrm{kg} \mathrm{L}^{-1}\right)$ nach der Vorernte und Ernte 2007 und nach fünf und acht Monaten Lagerung

Tabelle 10.39 Kaliumkonzentration [Mittelwert $(\bar{x})$ und Standartabweichung $\left(\sigma_{\mathrm{x}}\right)$ ] $\left(\mathrm{mg} \mathrm{kg}^{-1} \mathrm{FM}\right)$ in Sorten und Knollen verschiedener spezifischer Dichten $\left(\mathrm{kg} \mathrm{L}^{-1}\right)$ nach der Vorernte und Ernte 2006 und nach fünf und acht Monaten Lagerung

Tabelle 10.40 Kaliumkonzentration [Mittelwert $(\bar{x})$ und Standartabweichung $\left(\sigma_{\mathrm{x}}\right)$ ] $\left(\mathrm{mg} \mathrm{kg}^{-1} \mathrm{FM}\right)$ in Sorten und Knollen verschiedener spezifischer Dichten $\left(\mathrm{kg} \mathrm{L}^{-1}\right)$ nach der Vorernte und Ernte 2007 und nach fünf und acht Monaten Lagerung

Tabelle 10.41 Phosphorkonzentration [Mittelwert $(\bar{x})$ und Standartabweichung $\left(\sigma_{\mathrm{x}}\right)$ ] $\left(\mathrm{mg} \mathrm{kg}^{-1} \mathrm{FM}\right)$ in Sorten und Knollen verschiedener spezifischer Dichten $\left(\mathrm{kg} \mathrm{L}^{-1}\right)$ nach der Ernte 2006 und nach fünf und acht Monaten Lagerung

Tabelle 10.42 Phosphorkonzentration [Mittelwert $(\bar{x})$ und Standartabweichung $\left(\sigma_{\mathrm{x}}\right)$ ] $\left(\mathrm{mg} \mathrm{kg}^{-1} \mathrm{FM}\right)$ in Sorten und Knollen verschiedener spezifischer Dichten $\left(\mathrm{kg} \mathrm{L}^{-1}\right)$ nach der Ernte 2007 und nach fünf und acht Monaten Lagerung

Tabelle 10.43 Anteil Knollen ( $\left.\mathrm{g} \mathrm{kg}^{-1} \mathrm{FM}\right)$ verschiedener spezifischer Dichten $\left(\mathrm{kg} \mathrm{L}^{-1}\right)$ innerhalb einer Sorte nach der Ernte 2005 und nach fünf und acht Monaten Lagerung

Tabelle 10.44 Anteil Knollen ( $\left.\mathrm{g} \mathrm{kg}^{-1} \mathrm{FM}\right)$ verschiedener spezifischer Dichten $\left(\mathrm{kg} \mathrm{L}^{-1}\right)$ innerhalb einer Sorte nach der Ernte 2006 und nach fünf und acht Monaten Lagerung

Tabelle 10.45 Anteil Knollen ( $\left.\mathrm{g} \mathrm{kg}^{-1} \mathrm{FM}\right)$ verschiedener spezifischer Dichten $\left(\mathrm{kg} \mathrm{L}^{-1}\right)$ innerhalb einer Sorte nach der Ernte 2007 und nach fünf und acht Monaten Lagerung

Tabelle 10.46 Trockenmassekonzentration [Mittelwert $(\bar{x})$ und Standartabweichung $\left.\left(\sigma_{\mathrm{x}}\right)\right]\left(\mathrm{g} \mathrm{kg}^{-1} \mathrm{FM}\right)$ in Sorten und Knollen verschiedener spezifischer Dichten $\left(\mathrm{kg} \mathrm{L}^{-1}\right)$ nach der Ernte 2005 und nach fünf und acht Monaten Lagerung

Tabelle 10.47 Trockenmassekonzentration [Mittelwert $(\bar{x})$ und Standartabweichung $\left(\sigma_{\mathrm{x}}\right)$ ] $\left(\mathrm{g} \mathrm{kg}^{-1} \mathrm{FM}\right)$ in Sorten und Knollen verschiedener spezifischer Dichten $\left(\mathrm{kg} \mathrm{L}^{-1}\right)$ nach der Ernte 2006 und nach fünf und acht Monaten Lagerung 
Tabelle 10.48 Trockenmassekonzentration [Mittelwert $(\bar{x})$ und Standartabweichung $\left.\left(\sigma_{\mathrm{x}}\right)\right]\left(\mathrm{g} \mathrm{kg}^{-1} \mathrm{FM}\right)$ in Sorten und Knollen verschiedener spezifischer Dichten $\left(\mathrm{kg} \mathrm{L}^{-1}\right)$ nach der Ernte 2007 und nach fünf und acht Monaten Lagerung

Tabelle 10.49 Stärkekonzentration [Mittelwert $(\bar{x})$ und Standartabweichung $\left(\sigma_{\mathrm{x}}\right)$ ] $\left(\mathrm{g} \mathrm{kg}^{-1} \mathrm{FM}\right)$ in Sorten und Knollen verschiedener spezifischer Dichten $\left(\mathrm{kg} \mathrm{L}^{-1}\right)$ nach der Ernte 2005 und nach fünf und acht Monaten Lagerung

Tabelle 10.50 Stärkekonzentration [Mittelwert $(\bar{x})$ und Standartabweichung $\left(\sigma_{\mathrm{x}}\right)$ ] $\left(\mathrm{g} \mathrm{kg}^{-1} \mathrm{FM}\right)$ in Sorten und Knollen verschiedener spezifischer Dichten $\left(\mathrm{kg} \mathrm{L}^{-1}\right)$ nach der Ernte 2006 und nach fünf und acht Monaten Lagerung

Tabelle 10.51 Stärkekonzentration [Mittelwert $(\bar{x})$ und Standartabweichung $\left(\sigma_{\mathrm{x}}\right)$ ] $\left(\mathrm{g} \mathrm{kg}^{-1} \mathrm{FM}\right)$ in Sorten und Knollen verschiedener spezifischer Dichten $\left(\mathrm{kg} \mathrm{L}^{-1}\right)$ nach der Ernte 2007 und nach fünf und acht Monaten Lagerung

Tabelle 10.52 Konzentration des trockenen Zellwandmaterials [Mittelwert $(\bar{x})$ und Standartabweichung $\left.\left(\sigma_{\mathrm{x}}\right)\right]\left(\mathrm{g} \mathrm{kg}^{-1} \mathrm{FM}\right)$ in Sorten und Knollen verschiedener spezifischer Dichten $\left(\mathrm{kg} \mathrm{L}^{-1}\right)$ nach der Ernte 2005 und nach fünf und acht Monaten Lagerung

Tabelle 10.53 Konzentration des trockenen Zellwandmaterials [Einzelwerte] $\left(\mathrm{g} \mathrm{kg}^{-1} \mathrm{FM}\right)$ in Sorten und Knollen verschiedener spezifischer Dichten $\left(\mathrm{kg} \mathrm{L}^{-1}\right)$ nach der Ernte 2006 und nach fünf und acht Monaten Lagerung

Tabelle 10.54 Konzentration des trockenen Zellwandmaterials [Einzelwerte] $\left(\mathrm{g} \mathrm{kg}^{-1} \mathrm{FM}\right)$ in Sorten und Knollen verschiedener spezifischer Dichten $\left(\mathrm{kg} \mathrm{L}^{-1}\right)$ nach der Ernte 2007 und nach fünf und acht Monaten Lagerung

Tabelle 10.55 Pektinkonzentration [Mittelwert $(\bar{x})$ und Standartabweichung $\left(\sigma_{\mathrm{x}}\right)$ ] $\left(\mathrm{g} \mathrm{kg}^{-1} \mathrm{FM}\right)$ in Sorten und Knollen verschiedener spezifischer Dichten $\left(\mathrm{kg} \mathrm{L}^{-1}\right)$ nach der Ernte 2005 und nach fünf und acht Monaten Lagerung

Tabelle 10.56 Pektinkonzentration [Einzelwerte] $\left(\mathrm{g} \mathrm{kg}^{-1} \mathrm{FM}\right)$ in Sorten und Knollen verschiedener spezifischer Dichten $\left(\mathrm{kg} \mathrm{L}^{-1}\right)$ nach der Ernte 2006 und nach fünf und acht Monaten Lagerung 
Tabelle 10.57 Pektinkonzentration [Einzelwerte] ( $\left.\mathrm{g} \mathrm{kg}^{-1} \mathrm{FM}\right)$ in Sorten und Knollen verschiedener spezifischer Dichten $\left(\mathrm{kg} \mathrm{L}^{-1}\right)$ nach der Ernte 2007 und nach fünf und acht Monaten Lagerung

Tabelle 10.58 Pektinkonzentration [Mittelwert $(\bar{x})$ und Standartabweichung $\left(\sigma_{\mathrm{x}}\right)$ der Daten aus 2005, Einzelwerte der Daten aus 2006 und 2007] ( $\left.\mathrm{g} \mathrm{kg}^{-1} \mathrm{FM}\right)$ im Schalen- und Markgewebe nach der Ernte 2005, 2006 und 2007 und nach fünf und acht Monaten Lagerung der Sorten, deren Knollen nicht nach spezifischen Dichten getrennt wurden

Tabelle 10.59 Grad der Pektinveresterung [Mittelwert $(\bar{x})$ und Standartabweichung $\left(\sigma_{\mathrm{x}}\right)$ ] (\%) in Sorten und Knollen verschiedener spezifischer Dichten $\left(\mathrm{kg} \mathrm{L}^{-1}\right)$ nach der Ernte 2005 und nach fünf und acht Monaten Lagerung

Tabelle 10.60 Grad der Pektinveresterung [Einzelwerte] (\%) in Sorten und Knollen verschiedener spezifischer Dichten $\left(\mathrm{kg} \mathrm{L}^{-1}\right)$ nach der Ernte 2006 und nach fünf und acht Monaten Lagerung

Tabelle 10.61 Grad der Pektinveresterung [Einzelwerte] (\%) in Sorten und Knollen verschiedener spezifischer Dichten $\left(\mathrm{kg} \mathrm{L}^{-1}\right)$ nach der Ernte 2007 und nach fünf und acht Monaten Lagerung

Tabelle 10.62 Wetterdaten während der Vegetationsperioden in 2005, 2006 und $2007 \mathrm{im}$ Vergleich zu deren zehnjährigen Mittel von 1994 bis 2004 [Anzahl Sonnenschein-Stunden (SStd.) in einer Kalenderwoche (KW), Summe Sonnenschein-Stunden eines Monats ( $\Sigma$ SStd) im Kreis Soltau, durchschnittliche Temperatur in einer Kalenderwoche $\left({ }^{\circ} \mathrm{C}\right)$, durchschnittliche Temperatur eines Monats $\left(\mathrm{C}_{X}^{-}\right)$, Niederschlagssumme $(\Sigma \mathrm{mm})$ inklusive Beregung (kursiv) eines Monats in Dethlingen] und ausgewählte phänologische Entwicklungsstadien der Knollen, gekennzeichnet durch eine zweistellige BBCH-Codierung (Meier 2001) in denen u. a. die Krautabtötung (BBCH 48) und Ernte der Knollen (BBCH 99) als Vorernte (VE) und Ernte (HE) erfolgte

Tabelle 10.63 Ausgewählte Eigenschaften der untersuchten Sorten (Bundessortenamt 2004, 2005, 2006)

Tabelle 10.64 Standorteigenschaften und Informationen zum Anbau der Kartoffeln 148 


\section{Abkürzungsverzeichnis}

${ }^{1} \mathrm{O}_{2}$

singlet oxygen

Singulett-Sauerstoff

ABTS 2,29-azinobis (3-ethylbenzothiazoline-6-sulfonic acid) diammonium salt

B

boron

Bor

BSA Federal Plant Variety Office

Bundessortenamt

BSI blackspot index

Schwarzfleckigkeitsindex

$\mathrm{C}$

carbon

Kohlenstoff

$\mathrm{Ca} \quad$ calcium

Kalzium

CAT catalase

Katalase

$\mathrm{Cl} \quad$ chlorine

Chlor

$\mathrm{Cu}$

copper

Kupfer

CUPRAC copper (II) (or cupric) reducing antioxidant capacity

Kupfer-reduzierende antioxidative Kapazität

DP discolouration potential

oxidatives Potential

DPPH 2,2-diphenyl-1-picrylhydrazyl scavenging assay

Test zur Abnahme von 2,2-Diphenyl-1Picrylhydrazyl

$\mathrm{Fe} \quad$ iron

Eisen

FRAP ferric reducing ability of plasma

plasmatische Fähigkeit, Eisen zu reduzieren

\begin{tabular}{|c|c|c|}
\hline GAE & gallic acid equivalent & Gallussäureäquivalent \\
\hline $\mathrm{H}$ & hydrogen & Wasserstoff \\
\hline $\mathrm{H}_{2} \mathrm{O}_{2}$ & hydrogen peroxide & Wasserstoffperoxid \\
\hline $\mathrm{HO}^{-}$ & hydroxyl radical & Hydroxylradikal \\
\hline H-ORAC & hydroxyl radical averting capacity & $\begin{array}{l}\text { Kapazität zur Vermeidung einer Hydro- } \\
\text { xylradikalbildung }\end{array}$ \\
\hline HPLC & $\begin{array}{l}\text { high pressure liquid chroma- } \\
\text { tography }\end{array}$ & Hochdruckflüssigkeitschromatographie \\
\hline & potassium & Kalium \\
\hline
\end{tabular}




\begin{tabular}{|c|c|c|}
\hline $\mathrm{Mg}$ & magnesium & Magnesium \\
\hline $\mathrm{Mn}$ & manganese & Mangan \\
\hline Mo & molybdenum & Molybdän \\
\hline $\mathrm{N}$ & nitrogen & Stickstoff \\
\hline $\mathrm{Ni}$ & nickel & Nickel \\
\hline $\mathrm{O}$ & oxygen & Sauerstoff \\
\hline $\mathrm{O}_{2}^{-}$ & superoxide radical & Super- oder (Hyper-) oxidradikal \\
\hline $\mathrm{O}_{3}$ & ozone & Ozon \\
\hline ORAC & oxygen radical absorbance capacity & Kapazität, Sauerstoff zu binden \\
\hline $\mathrm{P}$ & phosphorus & Phosphor \\
\hline POD & peroxidase & Peroxidase \\
\hline PPO & polyphenol oxidase & Polyphenoloxidase \\
\hline PUFAs & poly-unsaturated fatty acids & mehrfach ungesättigte Fettsäuren \\
\hline $\mathrm{ROO}^{-}$ & peroxyl radikal & Peroxylradikal \\
\hline ROS & reactive oxygen species & freie Sauerstoffradikale \\
\hline $\mathrm{R}$ & gas constant & allgemeine Gaskonstante \\
\hline $\mathrm{Se}$ & selenium & Selen \\
\hline SOD & superoxide dismutase & Superoxiddismutase \\
\hline $\mathrm{T}$ & temperature & Temperatur \\
\hline TEAC & $\begin{array}{l}\text { trolox equivalent antioxidant capac- } \\
\text { ity }\end{array}$ & $\begin{array}{l}\text { Trolox entsprechende antioxidative } \\
\text { Kapazität }\end{array}$ \\
\hline TOSC & $\begin{array}{l}\text { Total oxyradical scavenging capaci- } \\
\text { ty }\end{array}$ & $\begin{array}{l}\text { Sauerstoffradikal-verringernde Ge- } \\
\text { samtkapazität }\end{array}$ \\
\hline TRAP & $\begin{array}{l}\text { total radical trapping antioxidant } \\
\text { parameter }\end{array}$ & $\begin{array}{l}\text { Gesamtradikalbindende antioxidative } \\
\text { Kenngröße }\end{array}$ \\
\hline Trolox & $\begin{array}{l}\text { 6-hydroxy-2,5,7,8- } \\
\text { tetramethylchoman-2-carboxylic } \\
\text { acid }\end{array}$ & \\
\hline $\mathrm{Zn}$ & zinc & Zink \\
\hline
\end{tabular}




\section{Vorwort}

Die Kartoffel (Solanum tuberosum) ist bereits seit Ende des 15. Jahrhunderts eine bekannte Kulturpflanze in europäischen Gärten. Ihre Verbreitung in den damalig feudalen deutschen Herrschaftsgebieten erfolgte u. a. während der zahllosen Feldzüge und Schlachten des Dreißigjährigen Krieges. Obwohl der Kartoffelanbau zur Nahrungsmittelproduktion teilweise befohlen wurde (wie 1756 von Friedrich II.), diente die Knolle bis 1770 fast ausschließlich als Viehfutter. Erst mit der Abschaffung der reinen Brache in den siebziger und achtziger Jahren des 18. Jahrhunderts und der reformierten preußischen Agrargesetzgebung begann der wirtschaftliche Kartoffelanbau, gefördert durch die fortschreitende Entwicklung von Kartoffelbrennereien (Schick und Klinkowski 1961). Im Jahr 1929 wurden in Deutschland etwa 2,8 Millionen ha der Ackerfläche mit Kartoffeln bepflanzt, nur Roggen und Hafer beanspruchten mehr Fläche (Hamester 1997). Da die Kartoffel überwiegend ein Grundnahrungsmittel der ländlichen Bevölkerung war (Schuhmann 1997), erfolgte deren Lagerung ebenfalls auf den Höfen in Kellern und Mieten (Pötke 2001). In dieser Zeit lagen die Erträge unter $100 \mathrm{dt} \mathrm{ha}^{-1}$ (Schuhmann 1997). Dabei handelten die Erzeuger mit bereits erwirtschafteten Überschüssen, indem sie die abgesackte Ware an Kommissionäre übergaben, die diese an städtische Gemüsefachgeschäfte und Fruchthandelsunternehmen verkauften (Hamester 1997). Der Transport erfolgte hauptsächlich auf dem Schienenweg, wobei die Knollen kaum bewegt wurden und daher keine Qualitätsprobleme auftraten (Pötke 2001). Der zunehmende züchterische Fortschritt, maschinelle Pflanzung, intensivere Bodennutzung und der Einsatz von Mineraldüngern im zweiten Drittel des 20. Jahrhunderts ermöglichten Erträge bis $200 \mathrm{dt} \mathrm{ha}^{-1}$ (Schuhmann 1997, Pötke 2001). Gleichzeitig erfolgten der Bau von Lagerhallen auf landwirtschaftlichen Betrieben und im Versorgungsbereich der Großstädte sowie eine Vergrößerung der Verkaufsflächen des Einzelhandels, was eine fortlaufende Versorgung der Bevölkerung mit Kartoffeln ermöglichte (Pötke 2001). Zunehmend traten Qualitätsmängel bei den Knollen auf, die in der Hauptsache Beschädigungen und Fäule, ausgelöst durch unpassende Transport- und Lagerbedingungen, betrafen (Wirsing 2001).

Seit dem letzten Drittel des 20. Jahrhunderts sind die Verfahrensschritte Anbau, Ernte, Transport, Lagerung und Aufbereitung bei der Kartoffelerzeugung durchgehend mechanisiert (Schuhmann 2001). Höhere Erträge von durchschnittlich $300 \mathrm{dt} \mathrm{ha}^{-1}$ und die Übernahme der Frühjahrs- und Winterversorgung der Bevölkerung mit Kartoffeln durch den Handel begünstigen den Bau von Großabpackanlagen (Pötke 2001), die zeitgemäß eine jahresunabhängige Bereitstellung von Kartoffeln in verschiedenen Gebinden ermöglichen (Hamester 1997). Im Vergleich zu früher üblichen Produktionsverfahren werden die Knollen gegenwärtig erheblich mechanisch belastet (Pötke 2001). Dabei tragen u. a. Material, Anzahl und Höhe der Fallstufen, Bandgeschwindigkeiten, Rollstrecken, Gutstrommenge, Gutstromlenkung und Relativbewegungen des Mechanisierungsmittels (Schuhmann 2001) und daraus resultierend die Belastungsstärke und die Anzahl der Stöße (Leppack 2001) zur 
potenziellen Beanspruchung der Knollen bei (Schuhmann 2001). Nach Boumann (1995) beeinflussen die einzelnen Verfahrensschritte Ernte, Lagerung, Aufbereitung und Verladen maßgeblich die Knollenqualität. Trotz vielseitiger technischer Fortschritte in allen Phasen des Produktionsprozesses ist es nicht möglich, den nachhaltigen Ertragssteigerungen entsprechende Qualitätsentwicklungen gegenüberzustellen. Obwohl äußere Knollenbeschädigungen und damit verbundene Verluste durch Fäulnisentwicklung verringert worden sind (Schuhmann 2001), bleibt die Qualität verkauffähiger Knollen vermindert, hervorgerufen durch häufiger auftretende innere Mängel, insbesondere durch Schwarzfleckigkeit (Wirsing 2001).

\section{Erscheinungsbild Schwarzfleckigkeit}

Im Gegensatz zu äußeren Beschädigungen sind innere Qualitätsmängel erst nach dem Schälen und/oder Schneiden der Knollen erkennbar, teilweise verbunden mit sichtbaren Gewebeverletzungen. Im Fall einer nicht sichtbaren Gewebeverletzung entwickeln sich oftmals weiß bis grau gefärbte Gewebeabschnitte in der Rindenschicht der Knollen, deren Farbe sich deutlich von der Farbe des übrigen Knollengewebes unterscheidet und mit zunehmender Zeit schwarz wird (McGarry et al. 1996). Nach drei bis sechs Wochen kann das verfärbte Gewebe als ein Bereich mehr oder weniger zerfallener Zellen erscheinen (Specht 1997). Diese Verfärbung des Gewebes wird als Schwarzfleckigkeit bezeichnet (McGarry et al. 1996). Die Intensität der Verfärbung wird u. a. durch die Art der mechanischen Belastung sowie durch Umweltfaktoren und die Variabilität von Sorten beeinflusst (Abb. 1.1, 1.2).

\section{Faktoren, die zur Ausprägung der Schwarzfleckigkeit beitragen}

Während der Ernte kann die Auswahl geeigneter Roder und deren fachgerechte Bedienung die mechanische Belastung der Knollen vermindern und somit deren Neigung zu Schwarzfleckigkeit mit bestimmen (Specht 1997, Peters 1999). Anschließende geringe Transportbewegungen zwischen dem Roden und den Abtrocknen der Knollen verringern zusätzlich potenzielle Beanspruchungen (Schuhmann 2001). Nach der Ernte wird die Qualität der Knollen u. a. durch die Transportbedingungen beeinflusst, wobei der Transport mehrere Etappen von der Lagerhalle des Erzeugers zu den Zwischen- und Aufbereitungsanlagen des Handels und weiter zu Zwischenlagern und Kaufhallen des Handels umfassen kann. Weitestgehend optimale Transport- und Aufbereitungsmaßnahmen, wie u. a. bei Schuhmann (2001) beschrieben, können helfen, das Risiko mechanischer Belastungen für die Knollen und somit deren Neigung zu Schwarzfleckigkeit zu verringern.

Die mechanische Belastbarkeit und daraus resultierende Neigung zu Schwarzfleckigkeit kann sich während der Lagerung ändern. Kartoffellagerung ist erforderlich, um eine ständige Versorgung mit frischer und verarbeitungsfähiger Ware $\mathrm{zu}$ ermöglichen. In einer Vielzahl von bisherigen Untersuchungen zum Einfluss der Lagerungsbedingungen auf das Auftreten der Schwarzfleckigkeit werden unterschiedliche Ergebnisse dargestellt. Eine Reihe von Arbeiten zeigen, dass während der Lagerung die Neigung zu Schwarzfleckigkeit 
erhöht wird (Dean et al. 1993, Sabba und Dean 1996, Edgell et al. 1998, Lærke 2001), während andere Autoren zu gegenteiligen Ergebnissen kommen (Workman und Holm 1984, Delgado et al. 2001, Lærke et al. 2002). Daneben werden auch sowohl zu- als auch abnehmende Schwarzfleckigkeitsneigungen in Abhängigkeit der Lagerdauer und untersuchten Sorte beschrieben (Heinecke 2007). McGarry (1996) erwähnt ebenfalls unterschiedliche Ergebnisse. Die Mehrzahl der Untersuchungen verweist auf physiologische Veränderungen der Knollen während der Lagerung, die eine veränderte Neigung zu Schwarzfleckigkeit bewirken können.

Verschiedene Umweltbedingungen während der Vegetation können ebenfalls die Neigung zu Schwarzfleckigkeit beeinflussen (McGarry 1996). Einige Untersuchungen zeigen, dass die sortenspezifische Neigung zu Schwarzfleckigkeit zwischen unterschiedlichen Vegetationsperioden entweder unverändert bleibt (Dean et al. 1993) oder verschieden sein kann (Workman und Holm 1984, Mondy und Munshi 1993, Lærke et al. 2002). Unterschiede scheinen überwiegend durch standortspezifische Faktoren wie Bodenart, Bodenwassergehalt und Nährstoffzufuhr (McNabnay 1999, van Loon und van den Berg 2003/4, Kirk et al. 2006) bedingt zu sein und können teilweise durch standortangepasste Anbaumaßnahmen (Bodenvorbereitung, Beregnung, Drainage, Düngung) ausgeglichen werden (Pawelzik und Delgado 1999, Thornton und Bohl 2000, Davenport et al. 2005). Jedoch ist der Einfluss dieser Maßnahmen auf die stoffliche Zusammensetzung der Knollen, welche die unterschiedlichen Neigung zu Schwarzfleckigkeit bewirkt (Kirk et al. 2006), in der Regel begrenzt (Thornton und Timm 1990).

Die stoffliche Zusammensetzung der Knollen wird jedoch von der Länge der Vegetationsperiode beeinflusst (Kolbe und Stephan-Beckmann 1997). Während Schuhmann (2001) die unbedingte sortentypische Ausreifung (nach chemischer Sikkation bei beginnender Krautvergilbung und bei Stärkegehalten der Knollen, die zu 80 \% dem sortentypischen Stärkegehalt entsprechen) der Knollen zur Erhaltung der Qualität einfordert, zeigen Untersuchungen von Pavek et al. (1985), Corsini et al. (1999), Thornton und Bohl (2000) sowie Kirk et al. (2006), dass eine vorzeitige Ernte der Knollen (nach chemischer Sikkation des grünen Krautes oder bevor 40 \% des Krautes gelb sind) deren Neigung zu Schwarzfleckigkeit verringern kann.

Kartoffelsorten neigen unterschiedlich zu Schwarzfleckigkeit (McNabnay et al. 1999, Wirsing 2001, Heinecke 2007). Die sortenspezifische Neigung zu Schwarzfleckigkeit wird als Schwarzfleckigkeitsindex (Schuhmann 2001) in der jährlich erscheinenden Beschreibenden Sortenliste des Bundessortenamtes berücksichtigt und spiegelt die zu erwartende Beschädigung der Knollen in allen Phasen des Produktionsprozesses wider, wobei der Schwarzfleckigkeitsindex keine genauen Aussagen über die Art und Höhe eventuell entstehender Qualitätsmängel ermöglicht (Leppack 2001).

Grundsätzlich zeigt sich, dass Knollen einer Kartoffelsorte (Abbildung 1.1) oder verschiedener Kartoffelsorten (Abbildungen 1.1 und 1.2) gegenüber einer definierten Belastung 
unterschiedlich reagieren können. Aus diesem Grund wird die Neigung zu Schwarzfleckigkeit vermutlich von mehreren Einflussfaktoren bestimmt (Thornton und Timm 1990).
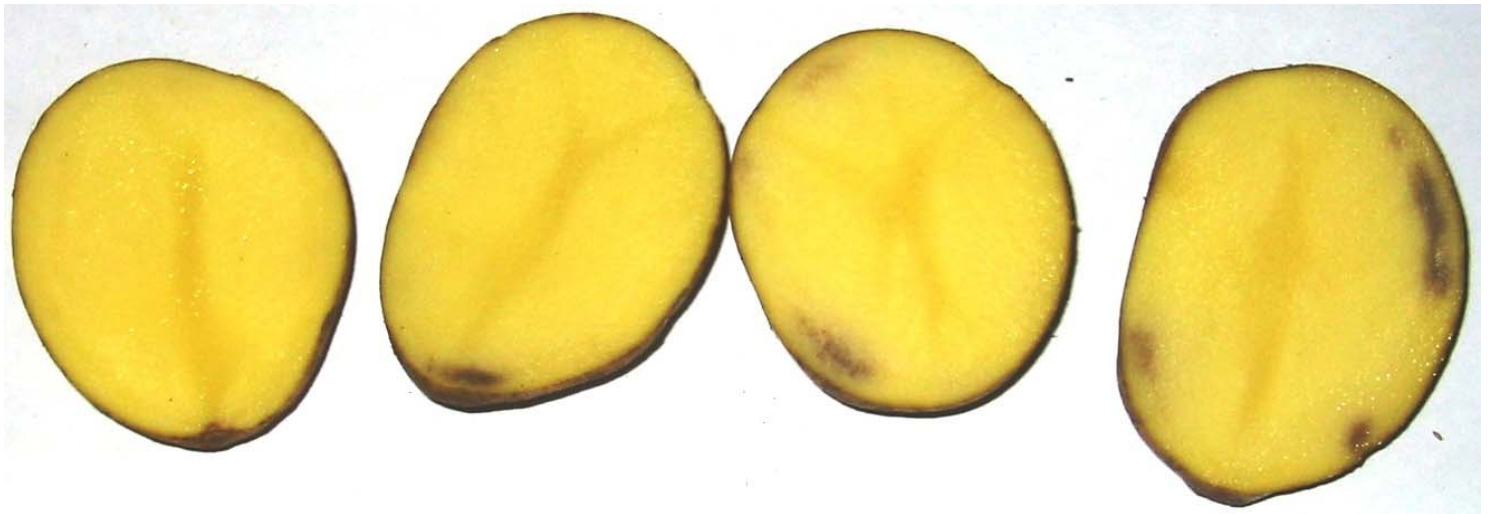

Abbildung 1.1 Knollen einer Kartoffelsorte (A) mit zunehmender Neigung zu Schwarzfleckigkeit (v.1.) nach der Ernte 2006

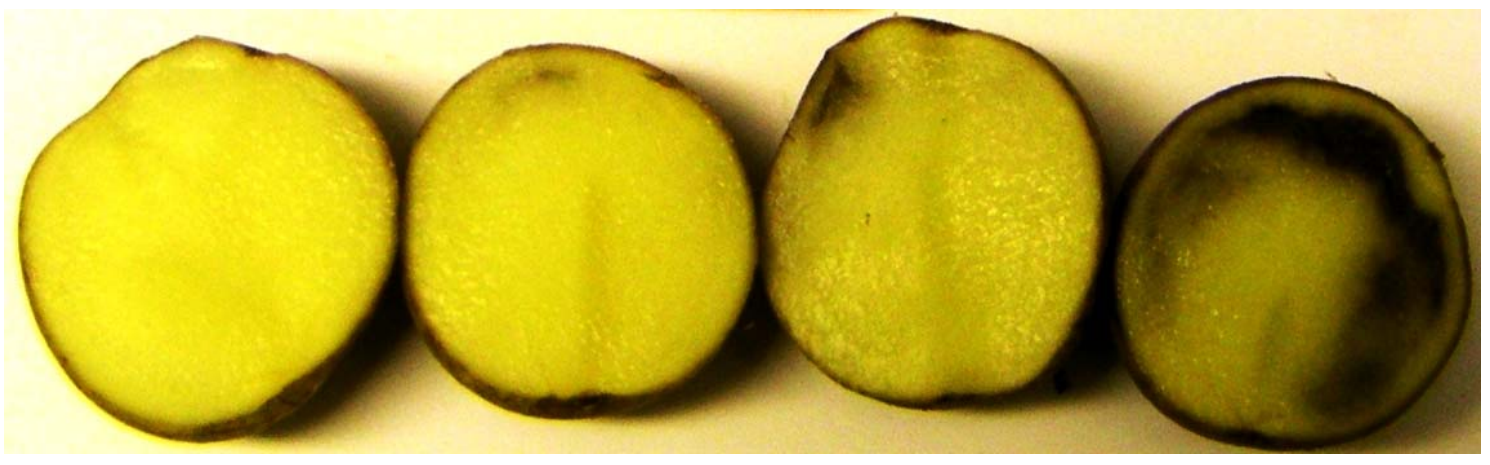

Abbildung 1.2 Knollen einer Kartoffelsorte (B) mit zunehmender Neigung zu Schwarzfleckigkeit (v.1.) nach der Ernte 2006

\section{Ziel der Arbeit}

Die vorliegende Arbeit beruht auf vielschichtigen Untersuchungen, die in ihrer ganzheitlichen Betrachtung zum generellen Verständnis der Reaktionsmechanismen der Schwarzfleckigkeit in Kartoffelknollen beitragen sollen. Basierend auf Erkenntnissen zu physiologischen Vorgängen in der Kartoffelknolle und unter Berücksichtigung guter fachlicher Praxis sollen zunächst kritische Punkte für die Entstehung von Schwarzfleckigkeit identifiziert werden, die zukünftig genutzt werden können, um Schwarzfleckigkeit bei Kartoffeln und daraus resultierende finanzielle Verluste nachhaltig zu reduzieren. Dazu lassen sich nachstehende einzelne Untersuchungsschwerpunkte formulieren, denen die in der vorliegenden Arbeit folgende Manuskripte (Kapitel 2 bis 5) gewidmet sind:

- Beitrag zur Aufklärung biochemischer Reaktionen, die Schwarzfleckigkeit fördern oder hemmen (Kapitel 2)

- Ermittlung des Einflusses von Umwelt-, Lager- und Sorteneigenschaften auf die Schwarzfleckigkeit (Kapitel 3) 
- Erkenntnisse über Verhältnisse einzelner Faktoren zueinander, die Schwarzfleckigkeit beeinflussen (Kapitel 4)

- Identifizierung einzelner Faktoren, die Schwarzfleckigkeit fördern oder hemmen (Kapitel 5)

Die Ergebnisse der Untersuchungen sollen in die gute fachliche Praxis integrierbar sein. Sie können den Kartoffelproduzenten weitere Möglichkeiten aufzeigen, durch angemessene Standortwahl, Anbaumaßnahmen (Düngung, Wasserversorgung) und Ernteverfahren die Neigung zu Schwarzfleckigkeit zu verringern. Für Lagerungs- und Abpackbetriebe sowie Händler sollen ebenfalls geeignete Bedingungen beschrieben werden, um die Neigung zu Schwarzfleckigkeit im Nacherntebereich zu minimieren. Für die Züchtung können die vorliegenden Ergebnisse Möglichkeiten aufzeigen, um die sortenspezifische Neigung zu Schwarzfleckigkeit genetisch zu beeinflussen.

Die vorliegende Arbeit ist in der Abteilung Qualität pflanzlicher Erzeugnisse am Department für Nutzpflanzenwissenschaften der Georg-August-Universität Göttingen im Rahmen eines Projektes angefertigt worden, das durch das Bundesministerium für Ernährung, Landwirtschaft und Verbraucherschutz, vertreten durch die Bundesanstalt für Landwirtschaft und Ernährung sowie durch die Gemeinschaft zur Förderung der privaten deutschen Pflanzenzüchtung finanziell gefördert wurde. Dabei erfolgte eine intensive Zusammenarbeit mit dem Institut für Sicherheit und Qualität bei Getreide des Max Rubner-Institutes Detmold, der Versuchsstation Dethlingen und dem Leibniz Institut für Agrartechnik Potsdam-Bornim.

Die nachstehenden Manuskripte werden bei verschiedenen Zeitschriften eingereicht und sind bereits nach deren Layoutvorgaben formatiert worden. 


\title{
2. Blackspot susceptibility in comparison with the antioxidant activity of potato (Solanum tuberosum) tubers
}

\begin{abstract}
Mechanical impact enhanced melanogenesis, which caused blackspot occurrence in potato tubers resulting in quality losses of fresh and processed products. Performed impact tests revealed the same blackspot susceptibility of cultivars after harvest in 2005 and after harvest in 2006. During a storage period of five months blackspot significantly decreased in tubers harvested in 2005 and contrary increased in tubers harvested in 2006. Long-term storage of eight months changed the blackspot susceptibility of tubers similar to freshly harvested tubers. Blackspot was significantly correlated with tuber specific gravity. Melanogenesis as a consequence of soluble oxidised substances was determined as discolouration potential. The obtained results pointed out that a cultivar specific periodical determination of its discolouration potential became essential to mirror their blackspot susceptibility. Present findings indicated that melanogenesis takes place as a part of antioxidant network or it is even a result. Some antioxidant systems were detected, which may avoid melanogenesis, respectively. The performed FRAP and H-ORAC- tests showed a slightly but significant correlation to mechanical stressed tubers.
\end{abstract}

Keywords: Solanum tuberosum, specific gravity, dormancy, melanogenesis, FRAP, H-ORAC

\section{Introduction}

After harvest potato tubers are exposed to mechanical impact. Insensitive handling operations as well as storage conditions and time influence the susceptibility of tubers to mechanical impact $(1,2)$. The level of initiation of mechanical stress is determined by genotypes and environmental conditions (3-5). Impact potentially increases the production of reactive oxygen species (ROS) $(6,7)$. The term describes free radicals (superoxide radical, $\mathrm{O}_{2}{ }^{--}$, peroxyl radical, $\mathrm{ROO}^{-}$and hydroxyl radical, $\mathrm{HO}^{-}$), as well as molecules like hydrogen peroxide $\left(\mathrm{H}_{2} \mathrm{O}_{2}\right)$, singlet oxygen $\left({ }^{1} \mathrm{O}_{2}\right)$ and ozone $\left(\mathrm{O}_{3}\right)(6)$. The function of the plant generated ROS is associated with the disease resistance mechanisms of cells (7) as well as the lipid peroxidation of polyunsaturated fatty acids (PUFAs) of cell membranes (9). Increasing generation of ROS under stress conditions induces both sub cellular damage and protective response (7). To protect cells against stress conditions the ROS level is controlled by an antioxidant system. In the cell, antioxidant compounds are closely linked with a powerful antioxidant activity. The effect based on their redox properties and is a result of various possible mechanisms like singlet-oxygen-quenching capacity, transition-metalchelating activity and free-radical scavenging action (8). Potato tissue contains several enzymes scavenging ROS [several types of superoxide dismutase (SOD), catalase (CAT), peroxidase (POD)] and a network of low molecular metabolites like phenolic compounds and vitamins $(6,8,9)$. Also the pigment melanin possesses antioxidative properties. Ac- 
cording to Hung et al. (10) numerous studies refer to the participation of melanin in freeradical reactions. At the stage of initiating chain reactions the function of the pigment might be tapping transitional metals or inactivating $\mathrm{O}_{2}^{-}$. Well, it is also known that the process of melanogenesis requires $\mathrm{O}_{2}^{-}(11)$ or even molecular oxygen $\left(\mathrm{O}_{2}\right)$ to oxidise phenolic substances to dopaquinone catalysed by the enzyme polyphenoloxidase (PPO) and finally polymerise to melanin $(2,12)$. In potato tubers dark melanin pigments develop under the intact skin after impact resulting in blackspot formation (2). This internal discolouration is a major quality problem for fresh and processing market worldwide.

To quantify the effect of harvesting and handling operations or of culture practice on melanogenesis different methods are described, e.g. pendulum, falling bolt and shaking procedures, which provide information about tissue firmness $(3,12,13)$. In the present study a mechanical load test was used. This test is similar to commercial situations but the control of the mechanical force is limited compared to other methods. Moreover, this method is time consuming because there is a time slot of 48 hours between mechanical treatment and visual evaluation. More rapid are methods which use photometric grading (1, $3,13,14)$. In general, these tests require homogenized material and the obtained results allow the quantification of the final optical density of the studied sample solutions or the rate of colour change over time. Via homogenization method the determination of the discolouration potential (DP) of potato tubers was practiced in the past $(3,13,14)$ and in the present study. With respect to the measurement of antioxidative compounds potentially responsible to prevent melanogenesis it is possible to measure all components individually, but as far as known antioxidants work additively and synergistically (15). Therefore single substance tests may not reflect the antioxidant functionality. Several analyses were developed to determine the combined antioxidant activity (16-19).

The objectives of this study were to test and compare several methods for the determination of blackspot susceptibility and to study the contribution of antioxidants (excepted melanin) in the blackspot susceptibility in potato tubers.

\section{Materials and methods}

Material. Eight cultivars of table potatoes (Adretta, Afra, Gala, Granola, Lolita, Marabel, Nicola and Renate) were grown near Dethlingen, Germany with conventional farming methods during two vegetation periods in 2005 and 2006. From harvested plants tubers with a similar size of 40 to $50 \mathrm{~mm}$ were selected to get comparable results. Analyses were performed in tubers directly after harvest and after five and eight months of storage at $4^{\circ} \mathrm{C}$ and $95 \%$ relative humidity, respectively. Tubers were divided into six groups of specific gravity from $<1.055$ to $>1.095 \mathrm{~kg} \mathrm{~L}^{-1}$, with 0.01 increments, whilst immersing them in vessels with demineralised water and different NaCl-concentrations. Subsequently samples were divided into two parts. Tubers from the first part were used to determine their blackspot index (BSI). Tubers from the second part were used for analysis. Also, nonfractionated tubers were peeled (1-2 mm thickness) using a hand peeler to obtain periderm 
and non-periderm tissue. Samples were sliced and used fresh or were shock-frozen in liquid nitrogen and stored at $-30{ }^{\circ} \mathrm{C}$ until testing or freeze-drying (Epsilon 2-40, Christ, Germany).

Analyses. Blackspot index (BSI) of whole potato tubers was determined according to the method of the German "Bundessortenamt" (BSA) (Federal Plant Variety Office). The tubers were cooled down to 4 to $5^{\circ} \mathrm{C}$ and mechanically treated for $50 \mathrm{~s}$ in a vegetable washing machine with a rotating drum (Flott $18 \mathrm{~K}$, Flottwerk H. J. Dames GmbH \& Co. KG, Rotenburg a. d. F., Germany) with three replications. Each replication consisted of an amount of tubers $\left(\Sigma_{\text {tuber }}\right)$ adequate to a volume of $6 \mathrm{~L}$. Afterwards, treated tubers were stored at room temperature for 48 hours. For BSI determination they were cut into halves length-wise and a half tuber was evaluated visually using a scale with four scores of discolouration: No discolouration indicated no melanin development and therefore no blackspot susceptibility $\left(\Sigma_{1}\right)$. Discolouration till quarter of length and less than $5 \mathrm{~mm}$ in-depth showed slight blackspot susceptibility $\left(\Sigma_{2}\right)$. Medium blackspot susceptibility corresponded to a discolouration till quarter of length and above $5 \mathrm{~mm}$ in-depth or a half of the length and maximal $5 \mathrm{~mm}$ in-depth $\left(\Sigma_{3}\right)$. Serious blackspot susceptibility was indicated by a stronger discolouration $\left(\Sigma_{4}\right)$. The blackspot susceptibility, expressed as the percentage of discoloured tubers was calculated from following equation:

$$
\text { BSI }(\%)=\left(0.3 \sum_{2}+0.5 \sum_{3}+\sum_{4}\right) / \sum_{\text {tuber }} * 100
$$

The discolouration potential (DP) of tubers was determined in fresh material using a homogenization method (14). Tubers differing in their specific gravity were analysed. According to Lærke et al. (1), Dean et al. (3) and Delgado et al. (13) stem end and bud end of tubers were mixed using an ultra-turrax (Janke \& Kunkel, IKA lab technics, Germany). Absorbance was measured at $475 \mathrm{~nm}$ with an UV-Vis spectral system (HP 8453, Germany). Results are the mean of three replications and expressed as absorbance units $\left(\mathrm{AU}_{475 \mathrm{~nm})}\right.$.

Investigation of the ferric reducing ability of plasma (FRAP) (20) was performed with tubers of different specific gravities, as well as with tubers separated in periderm and nonperiderm tissue. Sample preparation was done at $4^{\circ} \mathrm{C}$ by mashing the frozen samples in a cool mortar placed in iced water. Three phases of each sample were extracted to obtain material for the determination of free, cell wall associated compounds $(8,21,22)$ and water insoluble compounds (23), respectively. From $3 \mathrm{~g}$ of mashed sample the free compounds were extracted with $0.1 \mathrm{M} \mathrm{KH}_{2} \mathrm{PO}_{4}$ buffer ( $\mathrm{pH}$ 6.0). Double extraction was performed for cell wall associated compounds with $1 \mathrm{M} \mathrm{NaCl}$ solution. Water insoluble substances were extracted with n-hexane. Extracts were measured with an UV-Vis spectral system (HP 8453 , Germany) at $595 \mathrm{~nm}$. For comparison, standards of $1 \mathrm{mM} \mathrm{FeSO}_{4} * 7 \mathrm{H}_{2} \mathrm{O}$ solutions (20) were used, respectively solved in its extraction medium. The sum of the results represented the total FRAP (mmol kg-1 FM) of the sample. 
The hydroxyl radical averting capacity (H-ORAC) analysis followed Boxin et al. (24) and was carried out in freeze-dried samples of periderm and non-periderm tissue, which was mechanically ground to a fine powder. Sample extractions were performed in three replications. Hundred $\mathrm{mg}$ of a sample were mixed with $2 \mathrm{ml}$ solution of acetone: distilled water: acetic acid in the ratio 70: 29.5: 0.5 ( $\mathrm{pH}$ 5.0) for one hour. Afterwards, the mixture was centrifuged at $2660 \mathrm{~g}$ (Janetzki T-30, Germany) for 10 minutes and the clear supernatants were analysed. The calculation of relative H-ORAC based on gallic acid equivalents (GAE) per kilogram tuber fresh mass.

Statistics. The obtained data were analysed with Sigma Stat statistical program (STAT32). All data sets were tested for normal distribution and variance homogeneity $(p=0.05)$. Following analyses of variance were calculated for cultivar and specific gravity. Calculated means were compared by Tukey-test $(\mathrm{p} \leq 0.05)$. Correlation and multiple regression procedures between normally distributed quality parameters were performed using Pearson correlation coefficients.

\section{Results}

During the vegetation period of potatoes the specific gravity of tubers differs due to maturing time of cultivars, physiological tuber age, environmental conditions and pathogenic impact. Also tuber skin structures, gas concentration in the periderm and storage conditions affects tuber specific gravity (25). With respect to these facts potato tubers of the studied cultivars were separated according to their specific gravities after harvest and storage periods. Results of BSI, DP and FRAP tests are shown in Figure 2.1 and statistical evaluations of these tests are presented in Table 2.1 and in the annex (see Tab. 10.1, 10.2, 10.4, 10.5, $10.6,10.7,10.8,10.9,10.10)$. The standard deviations for BSI were not calculated because the required volume of tubers was not always available for all groups of specific gravity and it was therefore not possible to perform three replications. If the amount of tubers was less than a corresponding volume of 6 L the FRAP and DP test were performed only. In some cases, the standard deviation of FRAP and DP results tended to zero which is not visible in the graphs.

The BSI tests revealed a highly significant increasing blackspot susceptibility of tubers with increasing specific gravity after harvest 2005 and 2006 (Fig. 2.1, I, IV) and after five (Fig. 2.1, II, V) and eight months of storage (Fig. 2.1, III, VI), respectively. The FRAP activity tend to decrease with increasing specific gravity of the stored tubers (Fig. 2.1, III, VI). A significant correlation between BSI and DP was detected after harvest in 2005 and after five months of storage (Fig. 2.1, II, III), in tubers after harvest in 2006 and in stored tubers (Fig. 2.1, IV, V, VI), respectively. In general, both FRAP and DP values did not consistently represent the blackspot susceptibility of tubers differing in their specific gravity. 

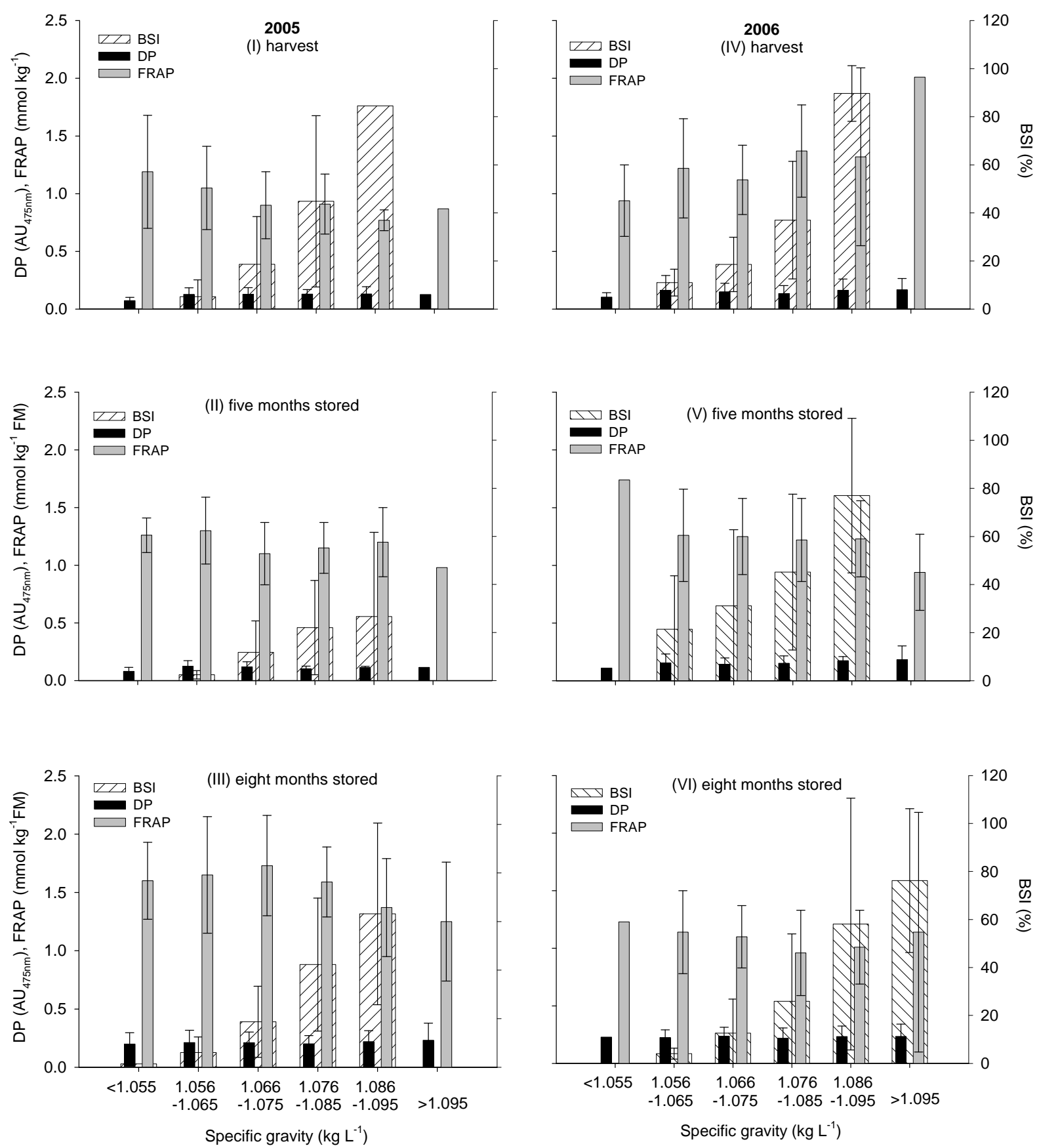

Figure 2.1 Blackspot index (BSI, \%) of potato tubers differing in their specific gravity $\left(\mathrm{kg} \mathrm{L}^{-1}\right)$ in comparison to their discolouration potential (DP, $\mathrm{AU}_{475 \mathrm{~nm}}$ ) and antioxidant activity (FRAP, mmol kg-1 FM) after harvest (I) in 2005, following five (II) and eight (III) months of storage, respectively and after harvest (IV) in 2006, following five (V) and eight (VI) months of storage, respectively, irrespective of cultivar, statistical evalutions are shown in Table 2.1 
Table 2.1 Relationships between blackspot susceptibility (BSI, \%), discolouration potential (DP, $\mathrm{AU}_{475 \mathrm{~nm}}$ ) and antioxidant activity (FRAP mmol kg${ }^{-1} \mathrm{FM}$ ) of tubers differing in their specific gravity $\left(\mathrm{kg} \mathrm{L}^{-1}\right)$ as Pearson Correlation Coefficient irrespective of cultivar, data refer to Figure 2.1

\begin{tabular}{lllll}
\hline Period & Parameter & BSI & DP & FRAP \\
\hline Harvest 2005 & Specific gravity & $0.556^{* *}$ & NS & $-0.261^{*}$ \\
& BSI & & $0.276^{*}$ & NS \\
& FRAP & & $0.347^{*}$ & \\
\hline Five months stored & Specific gravity & $0.472^{* *}$ & NS & NS \\
& BSI & & $0.287^{*}$ & NS \\
& FRAP & & NS & \\
\hline Eight months stored & Specific gravity & $0.762^{* *}$ & NS & $-0.410^{* *}$ \\
& BSI & & NS & $-0.543^{* *}$ \\
& FRAP & & NS & \\
\hline Harvest 2006 & Specific gravity & $0.756^{* *}$ & $0.398^{*}$ & $0.273^{*}$ \\
& BSI & & $0.644^{* *}$ & $0.357^{*}$ \\
& FRAP & & $0.544^{* *}$ & \\
\hline Five months stored & Specific gravity & $0.523^{* *}$ & $0.383^{*}$ & NS \\
& BSI & & $0.675^{* *}$ & $-0.389^{*}$ \\
& FRAP & & NS & \\
\hline Eight months stored & Specific gravity & $0.701^{* *}$ & NS & $-0.456^{* *}$ \\
& BSI & & $0.581^{* *}$ & NS \\
& FRAP & & $0.298^{*}$ & \\
\hline
\end{tabular}

${ }^{a}$ Abbreviations: NS, not significantly different; $* *$ significance at $\mathrm{p}=0.01, *$ significance at $\mathrm{p}=0.05$

Irrespective of the specific gravity of tubers the results of the BSI tests pointed out that the cvs. Afra, Adretta and Granola are medium to high susceptible to blackspot after harvest 2005 (Tab. 2.2). The cv. Renate showed a significantly lower blackspot susceptibility compared to cvs. Afra, Adretta and Granola. The cvs. Nicola, Lolita, Marabel and Gala were very low susceptible to blackspot which was significant different compared to Renate. The DP of cv. Adretta was the significantly highest compared to cvs. Granola, Afra, Lolita and Marabel. The FRAP activities of the cvs. Afra, Adretta and Granola were significant lower compared to cvs. Renate and Nicola. A relation to BSI was not observed because the less susceptible cv. Marabel showed also a low FRAP activity.

The storage period of five months significantly decreased the BSI of all cultivars. Contrary to these results, the data obtained with the DP method were similar compared to harvest evaluation. DP and FRAP results revealed no clear classification of cultivars with respect to their blackspot susceptibility.

After storage of eight months blackspot obtained by BSI test increased significantly in all cultivars when compared to the data obtained after five months of storage, regardless to specific tuber gravity. However, the BSI was not different compared to the results obtained 
after harvest. The DP increased significantly during eight months of storage in comparison to the DP obtained after harvest. The DP of cv. Adretta was the significantly highest compared to the other cultivars. The cvs. Granola, Renate and Nicola showed the same DP. In contrast, the FRAP values of cvs. Adretta, Granola and Afra were significant lower compared to cv. Lolita. The concentrations of antioxidant compounds were not different in cvs. Renate, Nicola, Marabel and Gala.

BSI test assessed on harvested tubers in 2006 indicated slight differences in blackspot susceptibility of cultivars compared with tubers harvested in 2005 (Tab. 2.2). In this year, the cv. Adretta was very susceptible to blackspot while the cv. Afra showed a medium susceptibility and cv. Granola was low susceptible. The DP method indicated again the significant highest values in cv. Adretta. With respect to their blackspot susceptibility a classification of cultivars after harvest on the basis of the DP tests was not possible because the obtained data were with exception of cv. Adretta not significantly different.

BSI test after five months of storage revealed that the blackspot susceptibility of tubers increased significantly, which was in contrast to the results obtained in 2005. However, the DP and FRAP values were not significantly different in stored cultivars compared with tubers after harvest.

With respect to eight months stored cultivars their BSI was not significantly different from tubers after harvest 2006. The DP increased significantly compared to the DP after harvest and five months of storage. With exception of cv. Adretta the DP of the cultivars were not significantly different. Long-term storage significantly decreased the FRAP activity of the presented cultivars. 
Table 2.2 Blackspot index (BSI, \%) of potato cultivars in comparison with their discolouration potential (DP, AU $475 \mathrm{~nm})$ and antioxidant activity (FRAP, mmol kg ${ }^{-1} \mathrm{FM}$ ) after harvest, five (5), and eight (8) months of storage of two vegetation periods (2005 and 2006) irrespective of their specific gravity

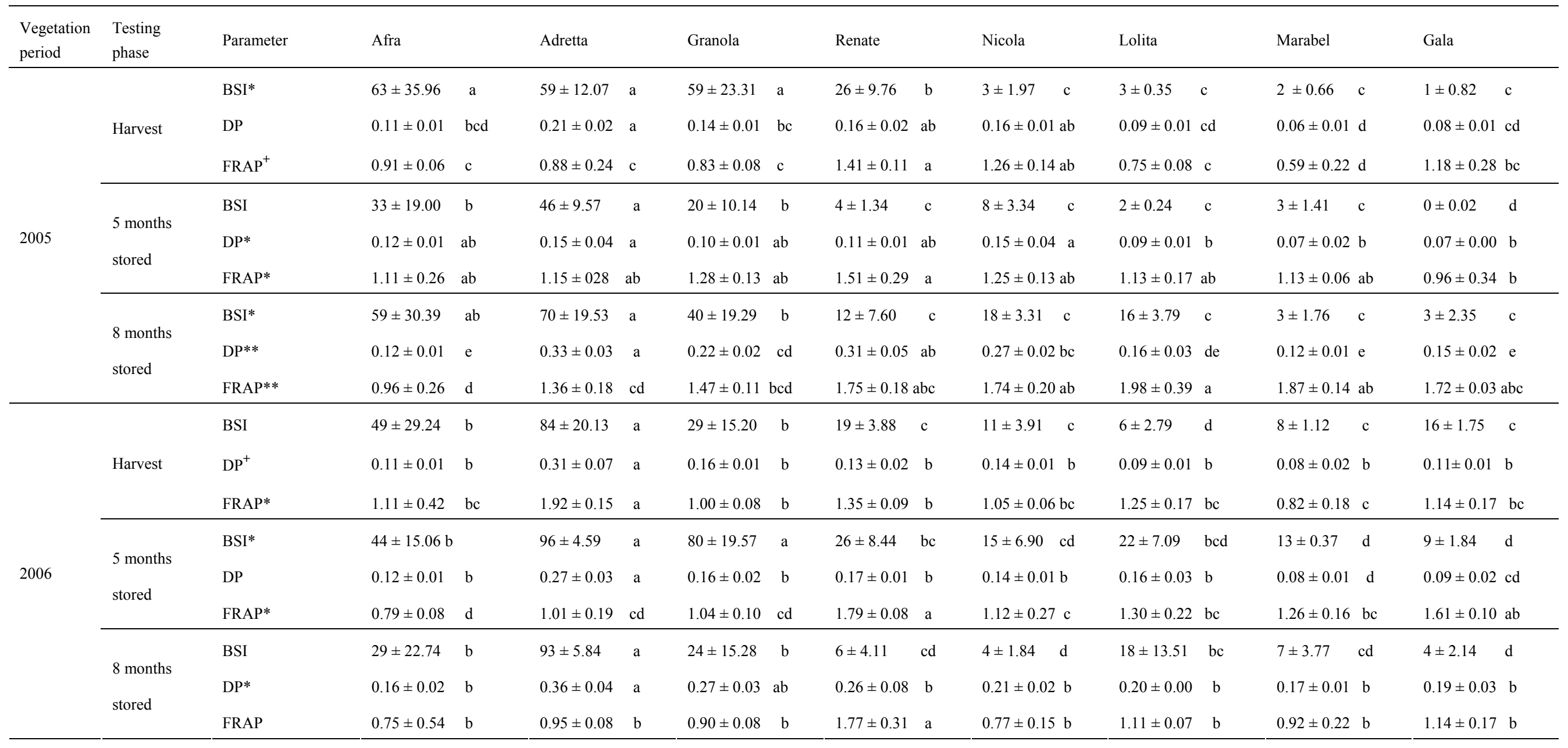

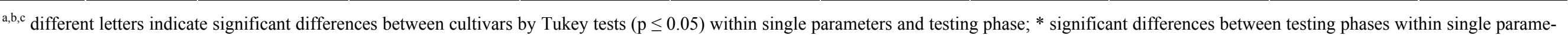
ters within one vegetation period by Tukey tests $(\mathrm{p} \leq 0.05){ }^{+}{ }^{+}$significant differences between vegetation periods within single parameters by Tukey tests $(\mathrm{p} \leq 0.05)$ 
Compared to whole tubers several reports attribute the highest antioxidant activity to the tuber periderm $(11,25)$. With respect to the formation of melanin pigments beneath the cortex tissue two methods were used to study the antioxidant activity of tuber periderm in comparison with tuber non-periderm and to study if a relationship with the BSI of whole tubers exists. The tubers were not separated in specific gravities before analysing (Tab. 2.3, 2.4). In tubers harvested in 2005 relative H-ORAC and FRAP activities revealed significant higher concentrations of antioxidant compounds in tuber periderm compared to nonperiderm concentrations. Cultivars with a higher antioxidant activity in periderm showed also a higher antioxidant activity in the non-periderm tissue. The relative H-ORAC compounds as a result of single tissue antioxidant activity were slightly more concentrated in tubers with a medium to high BSI of cvs. Adretta, Granola and Renate compared with cvs. Nicola, Lolita, Marabel and Gala $(r=0.401, \mathrm{p} \leq 0.05)$. Antioxidant activity determined with the FRAP method indicated significant lower concentrations of corresponding compounds in cvs. Granola and Marabel compared to the other cultivars (Tab. 2.3).

Table 2.3 Antioxidant activity (relative H-ORAC, mmol GAE kg-1 FM; FRAP, mmol kg-1 $\mathrm{FM}$ ) of tuber tissue of potato cultivars differing in their blackspot susceptibility (BSI, \%) harvested in 2005

\begin{tabular}{ll|l|l|l|l|l|l|l}
\hline & \multicolumn{3}{c}{ Relative H-ORAC } & \multicolumn{2}{c}{ Non-periderm } & \multicolumn{2}{c}{ Periderm* } & \multicolumn{2}{c}{ Whole tuber } \\
\cline { 2 - 9 } & \multicolumn{2}{c}{ Non-periderm } & \multicolumn{2}{c}{ Periderm* } & \multicolumn{2}{l}{ FRAP } \\
\hline Afra & $29.77 \pm 2.14$ & $38.15 \pm 3.25$ & $\mathrm{~cd}$ & $0.89 \pm 0.07$ & $1.39 \pm 0.28$ & $\mathrm{ab}$ & $83 \pm 7.05$ & $\mathrm{a}$ \\
Adretta & $42.09 \pm 3.53$ & $59.59 \pm 4.86$ & $\mathrm{a}$ & $1.00 \pm 0.05$ & $1.24 \pm 0.19$ & $\mathrm{ab}$ & $58 \pm 3.95$ & $\mathrm{~b}$ \\
Granola & $31.85 \pm 2.00$ & $57.94 \pm 5.80$ & $\mathrm{ab}$ & $0.64 \pm 0.18$ & $1.24 \pm 0.16$ & $\mathrm{~b}$ & $50 \pm 10.12$ & $\mathrm{c}$ \\
Renate & $42.88 \pm 9.53$ & $56.46 \pm 7.31$ & $\mathrm{a}$ & $1.13 \pm 0.34$ & $1.73 \pm 0.15$ & $\mathrm{a}$ & $39 \pm 11.59$ & $\mathrm{~d}$ \\
Nicola & $23.11 \pm 5.92$ & $30.99 \pm 3.37$ & $\mathrm{~d}$ & $0.86 \pm 0.16$ & $1.44 \pm 0.35$ & $\mathrm{ab}$ & $3 \pm 1.41$ & $\mathrm{f}$ \\
Lolita & $32.80 \pm 2.11$ & $48.21 \pm 2.29$ & $\mathrm{bc}$ & $0.72 \pm 0.03$ & $1.52 \pm 0.21$ & $\mathrm{ab}$ & $4 \pm 2.15$ & $\mathrm{e}$ \\
Marabel & $24.63 \pm 3.38$ & $47.41 \pm 0.83$ & $\mathrm{c}$ & $0.74 \pm 0.00$ & $1.13 \pm 0.12$ & $\mathrm{~b}$ & $2 \pm 0.91$ & $\mathrm{~g}$ \\
Gala & $33.55 \pm 4.61$ & $37.53 \pm 3.20$ & $\mathrm{~cd}$ & $1.18 \pm 0.12$ & $1.63 \pm 0.47$ & $\mathrm{a}$ & $2 \pm 0.83$ & $\mathrm{~h}$ \\
\hline
\end{tabular}

${ }^{a, b, c}$ different letters indicate significant differences of the whole tissue (on the basis of the sum of relative H-ORAC and FRAP values in non-periderm and periderm, respectively) of cultivars by Tukey tests $(\mathrm{p} \leq 0.05),{ }^{*}$ the periderm showed always significant higher H-ORAC-values and FRAP activity compared to the non-periderm tissue by Tukey tests $(\mathrm{p} \leq 0.05)$, (H-ORAC, hydroxyl radical averting capacity; GAE-equivalent, gallic acid equivalent; FRAP, ferric reducing ability of plasma; BSI, blackspot index)

Tubers harvested in 2006 were not significantly different in their BSI compared to tubers harvested in 2005 (Tab. 2.2). Again, the concentration of antioxidant compounds in tuber periderm was significantly higher than in tuber non-periderm (Tab. 2.4). However, the FRAP data and the relative H-ORAC data revealed significantly higher concentrations of antioxidant compounds in comparison with tubers harvested in 2005. The concentration of relative $\mathrm{H}-\mathrm{ORAC}$ antioxidants of periderm and non-periderm were slightly correlated with tubers BSI $(\mathrm{r}=0.514, \mathrm{p} \leq 0.05)$. 
Table 2.4 Antioxidant activity (relative H-ORAC, mmol GAE kg-1 FM; FRAP, mmol kg-1 $\mathrm{FM}$ ) of tuber tissue of potato cultivars differing in their blackspot susceptibility (BSI, \%) harvested in 2006

\begin{tabular}{|c|c|c|c|c|c|c|c|c|}
\hline & \multicolumn{3}{|c|}{ Relative H-ORAC } & \multicolumn{3}{|c|}{ FRAP } & \multicolumn{2}{|l|}{ BSI } \\
\hline & Non-periderm & Periderm* & & Non-periderm & Periderm* & & Whole tub & \\
\hline Afra & $47.01 \pm 7.16$ & $64.34 \pm 8.57$ & $\mathrm{bc}$ & $1.07 \pm 0.34$ & $1.43 \pm 0.15$ & $a b$ & $66 \pm 1.80$ & $\mathrm{~b}$ \\
\hline Adretta & $55.02 \pm 9.07$ & $109.47 \pm 6.34$ & $\mathrm{a}$ & $1.61 \pm 0.08$ & $2.04 \pm 0.13$ & $a b$ & $74 \pm 6.51$ & $\mathrm{a}$ \\
\hline Granola & $33.26 \pm 4.38$ & $75.74 \pm 1.87$ & $\mathrm{bc}$ & $1.26 \pm 0.03$ & $1.46 \pm 0.11$ & $\mathrm{ab}$ & $28 \pm 0.76$ & $\mathrm{c}$ \\
\hline Renate & $30.47 \pm 2.53$ & $41.93 \pm 3.39$ & $\mathrm{~d}$ & $1.70 \pm 0.07$ & $1.70 \pm 0.27$ & $\mathrm{a}$ & $20 \pm 5.24$ & d \\
\hline Nicola & $44.32 \pm 4.92$ & $65.81 \pm 3.95$ & bc & $1.16 \pm 0.31$ & $1.84 \pm 0.02$ & $\mathrm{ab}$ & $5 \pm 2.02$ & $\mathrm{~g}$ \\
\hline Lolita & $55.01 \pm 3.97$ & $72.41 \pm 2.63$ & $\mathrm{~b}$ & $1.01 \pm 0.20$ & $2.01 \pm 0.79$ & $\mathrm{a}$ & $5 \pm 1.02$ & $\mathrm{f}$ \\
\hline Marabel & $27.04 \pm 2.60$ & $76.33 \pm 4.33$ & $\mathrm{c}$ & $0.72 \pm 0.26$ & $1.09 \pm 0.07$ & $\mathrm{~b}$ & $10 \pm 2.75$ & $\mathrm{e}$ \\
\hline Gala & $45.16 \pm 3.05$ & $53.75 \pm 2.39$ & $\mathrm{c}$ & $0.89 \pm 0.04$ & $1.13 \pm 0.21$ & $\mathrm{~b}$ & $3 \pm 0.75$ & $\mathrm{~h}$ \\
\hline
\end{tabular}

a,b,c different letters indicate significant differences of the whole tissue (on the basis of the sum of relative H-ORAC and FRAP values in non-periderm and periderm, respectively) of cultivars by Tukey tests $(\mathrm{p} \leq 0.05),{ }^{*}$ the periderm showed always significant higher H-ORAC values and FRAP activity compared to non-periderm tissue by Tukey tests $(\mathrm{p} \leq 0.05)$ (H-ORAC, hydroxyl radical averting capacity; GAE-equivalent, gallic acid equivalent; FRAP, ferric reducing ability of plasma; BSI, blackspot index)

\section{Discussion}

BSI of freshly harvested tubers. The present investigation revealed a significant increase of BSI with increasing tuber specific gravity. This result supports the findings of Lærke (12) where tuber specific gravity of mechanical stressed cultivars may contribute to the level of energy introduced to the tuber by the impact. In our study, the specific gravity of tubers ranged between $<1.055$ to $>1.095 \mathrm{~kg} \mathrm{~L}^{-1}$. The cultivars can be characterized by cultivar-specific mean specific gravities. For example, the cvs. Afra, Adretta and Lolita performed many tubers with specific gravities from 1.075 to $1.085 \mathrm{~kg} \mathrm{~L}^{-1}$. However, their BSI was significantly different from each other. This observation was also done for cvs. Granola, Renate and Nicola with tuber mean specific gravity from 1.065 to $1.075 \mathrm{~kg} \mathrm{~L}^{-1}$. Tubers of cvs. Gala and Marabel showed a mean specific gravity from 1.055 to $1.065 \mathrm{~kg} \mathrm{~L}^{-1}$, whereby both cultivars had a low BSI. Results indicated that variation of tuber specific gravity within a cultivar determined its BSI. Present findings are comparable to studies of Wright et al. (26) who found that eight of ten tested cultivars showed a higher bruise score with increasing specific gravity. Both studies revealed that impact resulted in high correlations between blackspot susceptibility and tuber mass, irrespective of the applied test method. Specific gravity is generally assumed to be a cultivar characteristic (24) and reflects the dry matter concentration of potato tubers $(r=0.96)(27)$. The term dry matter refers to all substances of the potato tuber, except water (28) and includes $80 \%$ starch, $10 \%$ cell wall and $10 \%$ cytoplasmatic components (12). Lærke (12) described that cells fully filled with starch granules are more violable to impact bruise. High dry matter con- 
centrations may be a result of high numbers of starch granules which increase the potential of mechanical deformation to membranes as a direct effect of impact (12).

BSI of stored tubers. During storage, tubers undergo several stages of dormancy. The length of this period is controlled by genetic and environmental factors (29). At the end of dormancy, tubers turn into a source organ providing growth of the developing sprout (30). At that time, starch and protein degradation takes place and soluble sugars and amino acids become available (31). Prolonged storage initiates aging and senescence. Both processes are similar and the result of complex metabolic modifications. The senescence is characterized among others by increased activity of membrane associated enzymes (e.g. phospholipases), resulting in a release of membrane-bound PUFAs and their subsequent oxidation can lead to the generation of ROS. ROS are able to peroxidise amyloplast membrane lipids directly (31). Kumar \& Knowles (31) observed a degeneration of amyloplast membranes in relation to starch degradation during potato storage. Polyphenoloxydase (PPO) catalyses melanogenesis and is mainly located in the amyloplast. A relation to increasing BSI could be possible but direct relations were not reported. Lærke (12) pointed out that only few membrane studies focused on tonoplast degeneration revealed a related increase in blackspot susceptibility, where the tonoplast acts as physical barrier to pigment initiation. Therefore, age and metabolic status of tubers depending on genetic and environmental factors have an influence on the BSI of cultivars and tubers differing in their specific gravity during storage.

Tubers differing in their DP. The determination of blackspot susceptibility via DP showed a lower blackspot susceptibility of tubers harvested 2005 compared to tubers harvested 2006. The DP generally increased during storage. Relationships between BSI and DP were significant but the correlation coefficient was relatively low. Performed DP tests were suitable to recognize genotypic differences concerning blackspot susceptibility within a vegetation period, while DP methods did not consider physical properties. The present results show that the DP can not be used to compare blackspot susceptibility between different cultivars and growing periods. With respect to the method colour changes after 24 hours of whole unpeeled homogenised tubers was measured. The colour may occur as a mixture of actually originated melanin pigments and the cultivar characteristic tinct of periderm and non-periderm tissue. These findings are confirmed by Lærke et al. (1), who determined the DP of tubers in vivo using a colourimeter. Differences between BSI and DP may be due to the fact that pigment formation after impact takes place in one to three days in vivo but in only few minutes in vitro, which was also discussed by Lærke et al. (1) and Matheis and Belitz (32). Despite the fact that the potato tuber is well aerated in vivo discolouration may takes time because of a lack of oxygen.

Summarizing the data from vegetation and storage periods, blackspot susceptibility of the studied cultivars is closely connected to BSI tests. However, the results revealed only a weak relation between BSI and DP test. 
Antioxidant activity of tubers and tuber tissue. The deformation of the plasma membrane might potentially increase some levels of $\mathrm{ROS}$ (6). Some $\mathrm{ROS}$ like $\mathrm{HO}^{-}$and $\mathrm{O}_{2}{ }^{-}$react with the methylene group of PUFAs generating conjugated dienes, lipid peroxyl radicals and hydroperoxides (33). We assumed that lipid peroxidation might delayed by antioxidant compounds, which are able to advert $\mathrm{HO}^{-}$. In the present study, this ability was measured by the H-ORAC method. Moreover, antioxidants have ferric reducing abilities and might suspend branching of highly reactive peroxyl radicals (6), which was determined by the FRAP method. In this study, the antioxidant activity was significantly higher in periderm. Tuber periderm contains higher pectin concentration compared to non-periderm tissue (annex, Tab. 10.58), whereby phenolic compounds interacting with pectin polysaccharides (34) might be responsible for this higher antioxidant activity.

The relation between measured antioxidant activity and BSI was significant but not very high because both FRAP and H-ORAC methods did not determine the $\mathrm{O}_{2}{ }^{-}$scavenging ability. Singlet oxygen is more reactive than $\mathrm{H}_{2} \mathrm{O}_{2}$ and less reactive than $\mathrm{HO}^{-}$(6) and promote lipid peroxidation or is available for melanogenesis while FRAP and H-ORAC activity have taken place. One protection mechanism against $\mathrm{O}_{2}{ }^{-}$availability in higher concentrations may be the induction of superoxide dismutase (SOD), which catalyses the dismutation of $\mathrm{O}_{2}{ }^{-}$to $\mathrm{O}_{2}$ and $\mathrm{H}_{2} \mathrm{O}_{2}$. Furthermore, peroxidase (POD) and catalase (CAT) catalyse the breakdown of $\mathrm{H}_{2} \mathrm{O}_{2}$ to $\mathrm{H}_{2} \mathrm{O}$ and $\mathrm{O}_{2}$ (6). Nevertheless, both reaction pathways release $\mathrm{O}_{2}$, which also become shareable for melanogenesis. Eighter PPO and tyrosine are available and melanogenesis take place or remutated $\mathrm{O}_{2}$ will prolong lipid peroxidation or will be scavenged by low molecular mass antioxidants. $\mathrm{O}_{2}{ }^{-}$scavenging ability was detectable for low molecular mass antioxidants like quercitin $(35,36)$ and $\beta$-carotenoid $(33,38)$, which are significantly higher concentrated in pigmented potato tissue, compared to white tuber tissue $(9,19)$. Due to the fact that the dark yellow coloured tubers have higher $\beta-$ carotenoid content (37) a higher $\mathrm{O}_{2}^{-}$scavenging ability should be predictable resulting in lower blackspot susceptibility. Assuming this hypothesis for the present results of the study the dark yellow coloured cv. Gala revealed very low blackspot susceptibility throughout the period of investigation.

\section{Conclusion}

Potato cultivars grown in 2005 and 2006 differed in their blackspot susceptibility after harvest and after five and eight months of storage, respectively. During the entire period of investigation the BSI tests revealed physical tuber parameters related to the percentage of blackspot in potato tubers independent of pigment colour. The colour of the oxidized products was measured as DP. DP results were influenced by the natural colour of the tissue and therefore presented relationships between BSI and DP were significant but not very high. The DP did not show cultivar specific blackspot susceptibility when different cultivars were tested as reflected by the BSI test. Therefore the DP test is not practicable in order to compare the blackspot susceptibility of different cultivars. As an immediate con- 
sequence of impact physical and chemical tissue properties changed the balance of free ROS metabolism. ROS are involved in melanogenesis. Scavenging of ROS was realized by FRAP and H-ORAC mechanisms of antioxidants, particularly. Correlations between FRAP and BSI and H-ORAC and BSI were significant but not very high that indicated that ROS with high oxidation potential respond slightly to this mechanisms. Melanogenesis may be prolonged if required oxygen species will quenched by a combined non-enzymatic antioxidant network. The $\mathrm{O}_{2}{ }^{-}$scavenging ability of antioxidants may reflect an alternative way of melanogenesis in vitro. Available methods should be performed within potato tubers differing in their blackspot susceptibility.

\section{References}

(1) Lærke, P. E.; Christiansen, J.; Veierskov, B. Colour of blackspot bruises in potato tubers during growth and storage compared to their discolouration potential. Postharvest Biology and Technology. 2002, 26, 99-111.

(2) Stevens, L. H.; Davelaar, E. Isolation and Characterization of blackspot pigments from potato tubers. Phytochemistry. 1996, 42, 941-947.

(3) Dean, B. B.; Jackowiack, N.; Nagle, M.; Pavek, J.; Corsini, D. Blackspot pigment development of resistant and susceptible Solanum tuberosum L. genotypes at harvest and during storage measured by three methods of evaluation. American Potato Journal. 1993, 70, 201-217.

(4) Kirk, W. W.; da Rocha, A. B.; Hollosy, S. I.; Hammerschmidt, R.; Wharton, P. S. Effect of soil salinity on internal browning of potato tuber tissue in two soil types. Amer $J$ of Potato Res. 2006, 83, 223-232.

(5) Corsini, D.; Stark, J.; Thornton, M. Factors contributing to the blackspot bruise potential of Idaho Potato fields. Amer J of Potato Res. 1999, 76, 221-226.

(6) Blokhina, O.; Virolainen, E.; v. Fagerstedt, K. Antioxidants, oxidative damage and oxygen deprivation stress: a review. Ann Bot. 2003, 91, 179-194.

(7) Yahraus, T.; Chandra, S.; Legendre, L.; Low, P. S. Evidence for mechanically induced oxidative burst. Plant Physiol. 1995, 109, 1259-1266.

(8) Leo, L.; Leone, A.; Longo, C.; Lombardi, D. A.; Raimo, F.; Zacheo, G. Antioxidant compounds and antioxidant activity in "Early Potatoes". J Agric Food Chem. 2008, 56, 4154-4163.

(9) Stushnoff, C.; Holm, D.; Thompson, M. D.; Jiang, W.; Thompson, H. J.; Joyce, N. I.; Wilson, P. Antioxidant properties of cultivars and selections from the Colorado potato breeding program. Amer J of Potato Res. 2008, 85, 267-276.

(10) Hung, Y.-C.; Sava, V. M.; Makan, S. Y.; Chen, T.-H. J.; Hong, M.-Y.; Huang, G. S. Antioxidant activity of melanins derived from tea: comparison between different oxidative states. Food Chemistry. 2002, 78, 233-240. 
(11) Storey, M. The harvested crop. In Potato biology and biotechnology. Advances and perspectives, edition no 1; Vreugendhil, D. (Ed); Elsevier, Amsterdam, Netherlands, 2007; pp. 441-470.

(12) Lærke, P. E. Blackspot bruise in potato tubers: Ph. D. thesis, Department of Plant Biology, The Royal Veterinary and Agricultural University, Denmark. 2001, ISBN 8788976-50-5.

(13) Delgado, E.; Poberezny, J.; Pawelzik, E.; Rogozińska, I. Comparison of two methods for determining the discoloration potential of potato tubers based on their chemical and biochemical properties. Amer J of Potato Res. 2001, 78, 389-394.

(14) McNabnay, M.; Dean, B. B.; Bajema, R. W.; Hyde, G. M. The effect of potassium deficiency on chemical, biochemical and physical factors commonly associated with blackspot development in potato tubers. Amer J of Potato Res. 1999, 75, 53-60.

(15) Nzaramba, M. N.; Bamberg, J. B.; Miller (Jr) J. C. Effect of propagule type and growing environment on antioxidant activity ant total phenolics content in potato germplasm. Amer J of Potato Res. 2007, 84, 323-330.

(16) Pellegrini, N.; Serafini, M.; Colombi, B.; del Rio, D.; Salvatore, S.; Bianchi, M.; Brighenti, F. Total antioxidant capacity of plant foods, beverages and oils consumed in Italy assessed by three different in vitro assays. J. Nutr. 2003, 133, 2812-2819.

(17) Gorinstein, S.; Lojek, A.; Č́ž, M.; Pawelzik, E.; Delgado-Licon, E.; Medina, O. J.; Moreno, M.; Salas, I. A.; Goshev, I. Comparison of composition and antioxidant capacity of some cereals and pseudocereals. Int J Food Sci Tech. 2008, 43, 629-637.

(18) Yen, G-C.; Duh, P-D.; Tsai, H-L.; Antioxidant and pro-oxidant properties of ascorbic acid and gallic acid. Food Chem. 2002, 79, 307-313.

(19) Reddivari, L.; Hale, A. L.; Miller (Jr), J. C. Determination of phenolic content, composition and their contribution to antioxidant activity in specialty potato selections. Amer $J$ of Potato Res. 2007, 84, 275-282.

(20) Benzie, I. F. F.; Strain, J. J. The ferric reducing ability of plasma (FRAP) as a measure of antioxidant power: The FRAP assay. Anal Biochem. 1996, 239, 70-76.

(21) Civello, P. M.; Martínez, G. A.; Chaves, A. R.; Añón, M. C. Peroxidase from strawberry fruit (Fragaria ananassa Duch.): partial purification and determination of some properties. J Agric Food Chem. 1995, 43, 2596-2601.

(22) Keutgen, A. J.; Pawelzik, E. Modifications of strawberry fruit antioxidant pools and fruit quality under $\mathrm{NaCl}$ stress. J Agric Food Chem. 2007, 55, 4066-4072.

(23) George, B.; Kaura, C.; Khurdiya, D. S.; Kapoor, H. C. Antioxidant in tomato (Lycopersium esculentum) as a function of genotype. Food Chem. 2004, 84, 45-51. 
(24) Boxin, O.; Hampsch-Woodill, M.; Flanagan, J.; Deemer, E. K.; Prior, R. L.; Huang, D. Novel fluorometric assay for hydroxyl radical prevention capacity using fluorescein as the probe. J Agric Food Chem. 2002, 50, 2772-2777.

(25) Laboski, C. A. M.; Kelling, K. A. Influence of fertilizer management and soil fertility on tuber specific gravity: a review. Amer J of Potato Res. 2007, 84, 283-290.

(26) Wright, P. J.; Triggs, C. M.; Anderson, J. A. D. Effects of specific gravity and cultivar on susceptibility of potato (Solanum tuberosum) tubers to blackspot bruising and bacterial soft rot. New Zealand Journal of Crop and Horticultural Science. 2005, 33, 353361.

(27) Haase, N. U. Estimation of dry matter and starch concentration in potatoes by determination of under-water weight and near infrared spectroscopy. Potato Research. 2003/2004, 46, 117-127.

(28) Putz, B. Kartoffeln: Züchtung, Anbau, Verwertung. Verlag Behr's, Hamburg 1998.

(29) Suttle, J. C. Dormancy and Sprouting. In: Potato Physiology and Biotechnology. Advances and perspectives, edition no 1; Vreugdenhil, D. (Ed); Elsevier, Amsterdam, Netherlands, 2007; pp. 287-305.

(30) Sonnewald, U. Control of potato tuber sprouting. Trends Plant Sci. 2001, 6 (8), 333335.

(31) Kumar, M. G. N.; Knowles, N. R. Changes in lipid peroxidation and lipolytic and freeradical scavenging enzyme activities during aging and sprouting of potato (Solanum tuberosum) seed tubers. Plant Physiol. 1993, 102, 115-124.

(32) Matheis, G.; Belitz, H.-D. Untersuchungen zur enzymatischen Bräunung bei Kartoffeln (Solanum tuberosum). II. Quantitative Beziehung zwischen Bräunung und Inhaltsstoffen. Zeitschrift für Lebensmitteluntersuchung und -Forschung. 1977, 163, 186-190.

(33) Smirnoff, N. Antioxidant system and plant response to the environment. In: Environment and plant metabolism: flexibility and accumulation, edition no 1; Smirnoff, N. (Ed); BIOS Scientific Publishers, Oxford, UK, 1995 pp. 217-243.

(34) Sun-Waterhouse, D.; Melton, L. D.; O'Connor, C. J.; Kilmartin, P. A.; Smith, B. G. Effect of apple cell wall and their extracts on the activity of dietary antioxidants. J. Agric. Food Chem. 2008, 56, 289-295.

(35) Liu, C-L.; Chen, Y-S.; Yang, J-H.; Chiang, B-H. Antioxidant activity of Tartary (Fagopyrum tataricum (L.) Gaert.) and common (Fagopyrum esculentum Moench) Buckwheat sprouts. J. Agric. Food Chem. 2008, 56, 173-178.

(36) Lindberg Madsen, H.; Møller Andersen, C.; Viborg Jørgensen, L.; Skibsted, L. H. Radical scavenging by dietary flavonoids. A kinetic study of antioxidant efficiencies. European Food Research and Technology. 2000, 211, 240-246. 
(37) Klein, B. P.; Kurilich, A. C. Processing effects on dietary antioxidants from plant foods. Horticultural Science. 2000, 35, 580-584.

(38) Reyes, L. F.; Miller, J. C.; Cisneros-Zevallos, L. Antioxidant capacity, anthocyanins and total phenolics in purple and red-fleshed potato (Solanum tuberosum L.) genotypes. Amer J of Potato Res. 2005, 82, 271-277. 


\title{
3. Water relations in potato (Solanum tuberosum) tubers in comparison to their blackspot susceptibility
}

\begin{abstract}
Tubers harvested 2006 and 2007 and stored for five and eight months at low temperatures of $4^{\circ} \mathrm{C}$ avoided water losses and therefore losses of pressure potential due to cold accumulation which is known as a common practice of vegetative organs of carrots and radish. The performed mechanism of cold accumulation belonged to osmotic adjustment. Thereby blackspot susceptibility of tubers increased with increasing specific gravity, irrespective of whether cells were fully turgid or flaccid.
\end{abstract}

Keywords: water status, mechanical stress, cold storage

\section{Introduction}

Potato tubers are exposed to different environmental conditions during growth, harvest, handling and storage which predetermine their physiological status. These combinations of circumstances may affect the water status of the tuber as well as its variations due to metabolic changes and, as a consequence, can directly influence processing operations and product quality (Günel and Karadogan, 1998; Heuer and Nadler, 1998; Kolbe and StephanBeckmann, 1997; Pawelzik and Delgado, 1999; Rasool et al., 2007). In potato tubers blackspot is a major quality problem worldwide, caused by mechanical impact (Alvarez and Canet, 2002; Lærke, 2001). This blue-black discolouration of cortical parenchyma (Lulai, 2007) occurs without recognizable cell dissociation (Storey, 2007) contrary to other types of bruise like crushing, shatter and cracking leading to visible cell deformation (Baritelle and Hyde, 2003). However, intracellular membrane modification might be necessary to synthesize the dark polyphenolic pigment melanin from phenols stored in the vacuole catalysed by the amyloplast-located (Lærke et al., 2002) enzyme Polyphenol oxidase (PPO, EC 1.10.3.1, EC 1.14.18.1) (Schmitz et al., 2008). It is highly probable that tuber water status affects blackspot susceptibility (Bajema et al., 1998a; Baritelle and Hyde, 2001; Corsini et al., 1999; Lærke, 2001; Olsen et al., 2003/4; Pawelzik and Delgado, 1999) where water loss change membrane integrity allowing enzymatic browning as obtained in rambutan fruits (Nephelium lappaceum) (Landrigan et al., 1996). Moreover blackspot development is interrelated with many other parameters concerning potato tubers impact susceptibility (e.g. tuber mass, tuber shape, physiological tuber age) (Storey, 2007).

In the past, many studies investigated single features of tissue water status and their interactions with tuber mechanical properties like pressure potential and impact susceptibility of potato tubers (Alvarez et al., 2000a; Bajema et al., 1998a; Lærke, 2001), elasticity and specific gravity of potato parenchyma (Scanlon et al., 1998) and cold storage and impact susceptibility of potato tubers (Bajema et al., 1998a, b). However, tissue water status of the tuber is related to both its water concentration (e.g. water mass or water volume) and the chemical potential of the water within cells (Galindo et al., 2004b; von Willert et al., 
1995). The latter is commonly described by the actual tissue water potential, which, itself is determined by the respective osmotic (osmotic potential) and pressure component (pressure potential or turgor) (Galindo et al., 2004b). Detailed information about the chemical potential of water and impact susceptibility was given for carrot roots (Herppich et al., 1999) and for radish tubers (Landahl et al., 2004) and particularly for potato tubers (Lærke, 2001).

The present investigation evaluates the chemical potential of water in potato tubers to provide detailed measurements of changes in water status through time and to relate these changes, if any, to blackspot susceptibility of potato tubers. This study is aimed to get a more exact knowledge about blackspot development and may facilitate to develop optimal techniques of handling and storage which help to obtain and to maintain the highest possible product quality for the consumer.

\section{Materials and methods}

Material. Eight cultivars of table potatoes (Adretta, Afra, Gala, Granola, Lolita, Marabel, Nicola and Renate) were grown near Dethlingen (Germany) by conventional farming methods during two vegetation periods (2006 and 2007). To guarantee comparable results only tubers with a similar size of 40 to $50 \mathrm{~mm}$ were chosen for the experiments. Analyses were performed directly after harvest and after five and eight months, respectively, of storage at $4^{\circ} \mathrm{C}$ and $95 \%$ relative humidity. Prior to investigation tubers were sorted into six classes of specific gravity from $<1.055$ to $>1.095 \mathrm{~kg} \mathrm{~L}^{-1}$, with 0.01 increments, by immersing them into $\mathrm{NaCl}$-solutions of different concentrations. Then, the tubers of each class were split into two batches. Tubers of the first batch were used to determine their blackspot index (BSI), those of the second batch were used to determine water concentration, water potential, osmolality, osmotic potential, modulus of elasticity and pressure potential in the stem end and the bud end of tubers, respectively.

Analyses. Blackspot index (BSI) of whole potato tubers of different specific gravities was determined according to the method propose by the "Bundessortenamt" (BSA, Federal Plant Variety Office, Germany). The tubers were cooled down to 4 to $5^{\circ} \mathrm{C}$ and mechanical treated for $50 \mathrm{~s}$ in a vegetable washing machine with rotating drum (Flott $18 \mathrm{~K}$, Flottwerk H.J. Dames GmbH \& Co. KG, Rotenburg a. d. F., Germany) with three replications. Each replication consisted of an amount of tubers $\left(\Sigma_{\text {tuber }}\right)$ adequate to a volume of 6 L. Subsequently, treated tubers were stored at room temperature for 48 hours. For BSI determination tubers were cut into halves length-wise and one half was evaluated visually using a scale with four scores of discolouration. The score "No discolouration" indicated the lack of any blackspot development and therefore no blackspot susceptibility $\left(\Sigma_{1}\right)$. Discolouration of up to one quarter of length and less than $5 \mathrm{~mm}$ in depth denoted slight blackspot susceptibility $\left(\Sigma_{2}\right)$. Medium blackspot susceptibility corresponded to a discolouration of up to one quarter of length and more that $5 \mathrm{~mm}$ in depth or one half of the length and a maximum of $5 \mathrm{~mm}$ in depth $\left(\Sigma_{3}\right)$. Serious blackspot susceptibility was indicated by any discol- 
ouration stronger than that described before $\left(\Sigma_{4}\right)$. The blackspot susceptibility (BSI), expressed as the percentage of discoloured tubers, was calculated as $\left(0.3 \Sigma_{2}+0.5 \Sigma_{3}+\Sigma_{4}\right) / \Sigma_{\text {tuber }} *$ 100 .

Water relation parameters of tubers of the second batch were determined on tissue discs (6 $\mathrm{mm}$ diameter and $2 \mathrm{~mm}$ thick) from stem end and bud end of the tuber. With a cork borer tissue cylinders were axially cut from the middle of the tubers. The analysed samples consisted of both cortex and parenchyma tissue, which is important because blackspot occurs in the vascular bundles and parenchyma tissue under the cortex.

Water potential of the tissue discs was determined psychrometrically (von Willert et al., 1995) using $10 \mathrm{C}-52$ dew point hygrometer chambers connected to a HR-33T micro voltmeter via a PS-10 switchbox (all Wescor Inc., Logan, USA). The samples were inserted into the deep sample holders of the C-52 chambers and equilibrated for $60 \mathrm{~min}$ in the closed chambers. Subsequently, the discs were removed from the chambers, their fresh mass (FM) obtained (electronic balance BP 210 S, Satorius AG, Goettingen, Germany), and the discs were allowed to transpire freely for $30 \mathrm{~min}$. Afterwards, the water potential and fresh mass were measured again.

Finally, samples were filled into pre-weighted 1,5 $\mathrm{ml}$ micro test tubes (Eppendorf AG, Hamburg, Germany), frozen, thawed, centrifuged for 3 min (14000g, MiniSpin ${ }^{\circledR}$ plus, Eppendorf AG, Hamburg, Germany) and the supernatant analysed for its molal osmotic concentration $\left(c_{\text {osm }}\right.$; mmol kg ${ }^{-1}$ ) with a vapour pressure osmometer (VAPRO 5520, Wescor Inc., Logan, USA). The osmotic potential $\left(\Psi_{\pi}\right)$ was calculated from the osmotic concentration using the van't Hoff's relation $\left(\Psi_{\pi}=c_{\mathrm{osm}}{ }^{*} \rho_{\text {solution }}{ }^{*} \mathrm{R} * \mathrm{~T}\right.$; c.f. von Willert et al., 1995). From the difference of water potential and osmotic potential, pressure potential $\left(\Psi_{\mathrm{P}}\right)$ was obtained. Finally tissue dry mass (DM, $\left.\mathrm{g} \mathrm{kg}^{-1} \mathrm{FM}\right)$ was obtained after oven-drying at $85^{\circ} \mathrm{C}$ (24 hours) and the fresh mass based water concentration of the discs was calculated from fresh and dry mass ((FM-DM)/FM*1000). From changes in pressure potential and fresh mass during the two subsequent measurements multiplied by the initial fresh mass $\left(\mathrm{FM}_{\mathrm{i}}\right)$ the volumetric modulus of elasticity ( $\varepsilon$, c.f. von Willert et al., 1995) of the tissue samples was calculated according to Landahl et al. (2004) as $\left.\varepsilon=\left(\Delta \Psi_{\mathrm{P}}\right) / \Delta \mathrm{FM}\right) * \mathrm{FM}_{\mathrm{i}}$.

Statistics. The obtained data were analysed with Sigma Stat statistical program (STAT32). All data sets were tested for normal distribution and variance homogeneity $(p=0.05)$. Following analyses of variance were calculated for cultivar and specific gravity. Calculated means were compared by Tukey's test $(\mathrm{p}<0.05)$. Correlation and multiple regression procedures between normally distributed quality parameters were performed using Pearson correlation coefficients.

\section{Results}

As the stem end of potato tubers is physiologically and histologically older than the bud end (Bajema et al., 1998b) and might display some differences in both physiological prop- 
erties and blackspot susceptibility (Lærke, 2001) water relation and tissue elasticity were analysed separately for both tuber parts. However, no significant differences in water potential, osmolality, osmotic potential, pressure potential and the modulus of elasticity between both parts could be revealed (annex, Tab. 10.16, 10.19, 10.22, 10.25, 10.28). Hence, data of stem and bud ends of tubers were pooled. Also, the water potentials did not differ significantly between cultivars. Therefore, presented data reflect the mean water potentials of both stem end and bud end tissue of a selection of tubers differing in their specific gravity regardless of the respective cultivar (Tab. 3.1, 3.2).

Tubers harvested in 2006 showed specific gravities in the range of 1.055 and $1.095 \mathrm{~kg} \mathrm{~L}^{-1}$ (Tab. 3.1). Their absolute water concentration was negatively correlated with specific gravity $(\mathrm{r}=-0.86, \mathrm{p}<0.01)$, and blackspot occurrence $(\mathrm{r}=-0.59, \mathrm{p}<0.01)$ (Fig. 3.1A). However, blackspot significantly increased with increasing specific gravity $(r=0.79, p<0.01)$. Neither water potential nor osmolality or osmotic potential, pressure potential and modulus of elasticity varied significantly between tubers with differing specific gravity (Tab. 3.1).

After five months of storage, the variation of specific gravity increased now ranging between $<1.055$ and $>1.095 \mathrm{~kg} \mathrm{~L}^{-1}$ (Tab. 3.1). A five months storage period did not influence the tuber water concentration, although the correlation between absolute water concentration and specific gravity $(r=0.79, \mathrm{p}<0.01)$, water concentration and blackspot $(\mathrm{r}=0.50, \mathrm{p}$ $<0.05$ ) (Fig. 3.1B) and blackspot and specific gravity $(r=0.52, p<0.05)$ was still high. During storage pressure potential and the modulus of elasticity remained constant, while water potential and osmotic potential decreased significantly (Tab. 3.1). In contrast, a significant increase in osmolality and blackspot occurred (Tab. 3.1).

During further storage up to eight months the tuber water concentration and water potential did not change further. Again, the correlation between water concentration and specific gravity $(r=0.88, p<0.01)$, water concentration and blackspot $(r=0.62, p<0.01)$ (Fig. 3.1C) and blackspot and specific gravity $(\mathrm{r}=0.57, \mathrm{p}<0.01)$ was obvious. Compared to initial storage osmolality, modulus of elasticity and pressure potential significantly increased, while the osmotic potential and blackspot occurrence significantly decreased (Tab. 3.1).

In contrast to 2006, in 2007 specific gravities of harvested tubers ranged between $<1.055$ and $>1.095 \mathrm{~kg} \mathrm{~L}^{-1}$ (Tab. 3.2). In addition the water concentration was negatively correlated with specific gravity $(r=-0.89, \mathrm{p}<0.01)$, and blackspot $(\mathrm{r}=-0.56, \mathrm{p}<0.01)$ (Fig. 3.1D). Again, blackspot occurrence significantly increased with increasing specific gravity $(\mathrm{r}=$ $0.58, \mathrm{p}<0.01)$. Water potential, osmolality and osmotic potential were not significant different in tubers differing in their specific gravity. However, the modulus of elasticity and pressure potential was significantly lower in tubers with a specific gravity above $1.095 \mathrm{~kg}$ $\mathrm{L}^{-1}$ (Tab. 3.2). Specific gravity and water concentration of tubers were more or less constant during a storage of five months (Tab. 3.2). Again water concentration and specific gravity $(r=0.92, p<0.01)$ and blackspot and specific gravity $(r=0.52, p<0.05)$ were highly correlated but not water concentration and blackspot (Fig. 3.1E). Water potential, osmotic 
potential, modulus of elasticity and blackspot occurrence significantly decreased, while the pressure potential and osmolality significantly increased during initial storage (Tab. 3.2).

During a prolonged storage period up to eight months tubers specific gravity, water concentration, osmotic potential and modulus of elasticity of tubers stayed constant, while a correlation between absolute water concentration and specific gravity $(\mathrm{r}=0.66, \mathrm{p}<0.01)$ and blackspot and specific gravity $(\mathrm{r}=0.56, \mathrm{p}<0.01)$ was still given. Contrary to 2006 , no relationship between absolute water concentration and blackspot was detectable (Fig. 3.1F) in 2007. During long-term storage the osmolality significantly increased, while pressure potential and blackspot occurrence significantly decreased (Tab. 3.2). 

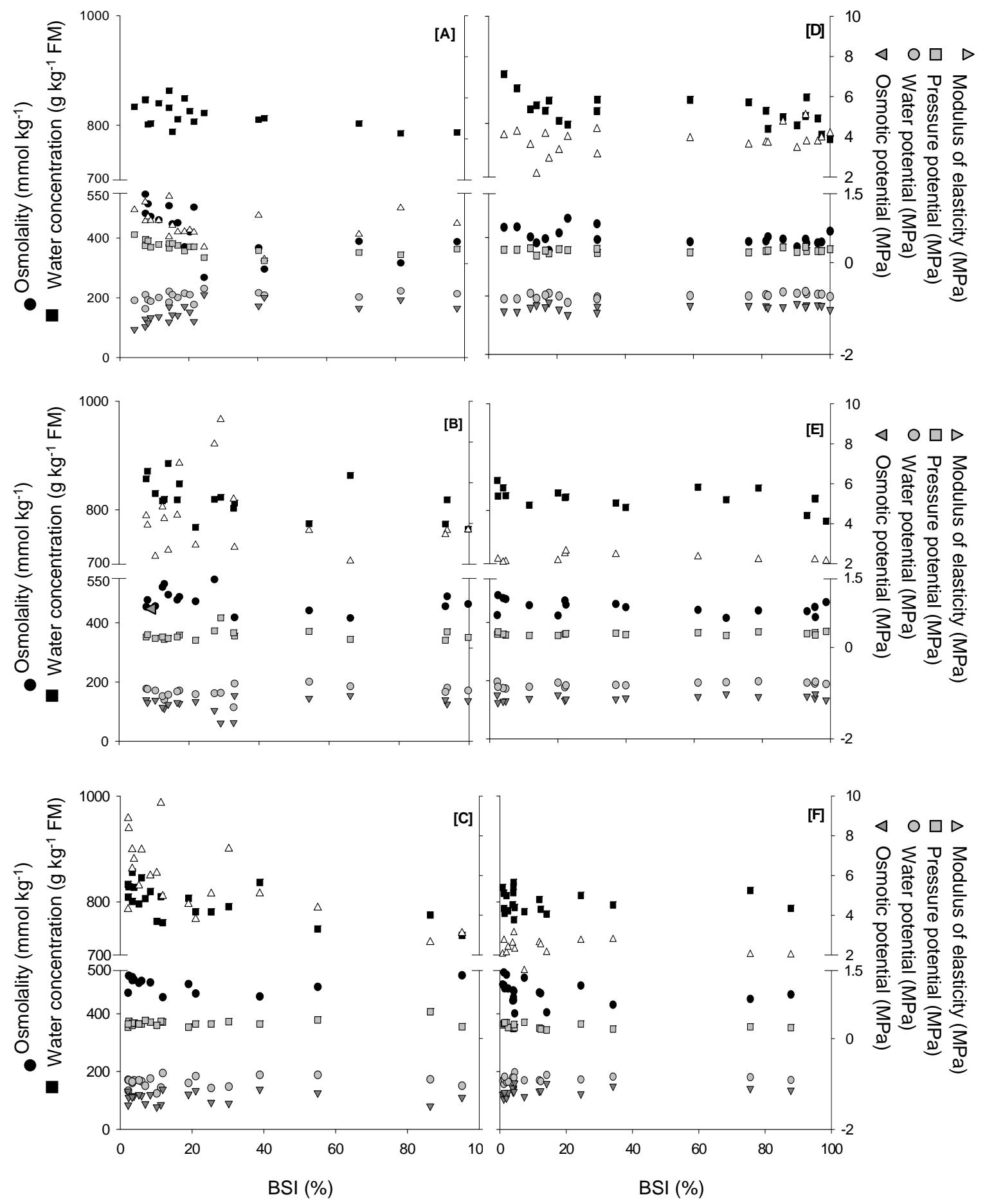

Figure 3.1 BSI (\%) of potato tubers of different specific gravities in comparison to their water concentration $\left(\mathrm{g} \mathrm{kg}^{-1} \mathrm{FM}\right)$, osmolality ( $\left.\mathrm{mmol} \mathrm{kg}^{-1}\right)$, osmotic potential (MPa), water potential $(\mathrm{MPa})$, pressure potential $(\mathrm{MPa})$ and modulus of elasticity $(\mathrm{MPa})$ after harvest (A) 2006, following five (B) and (C) eight months of storage, respectively, and after harvest (D) 2007, following five (E) and eight (F) months of storage, respectively, statistical evaluations are shown in Table 3.1 and 3.2 
Table 3.1 Water concentration $\left(\mathrm{g} \mathrm{kg}^{-1} \mathrm{FM}\right)$, water potential (MPa), osmolality ( $\left.\mathrm{mmol} \mathrm{kg}^{-1}\right)$, osmotic potential (MPa), modulus of elasticity (MPa), pressure potential (MPa) and BSI (\%) of tubers differing in their specific gravity $\left(\mathrm{kg} \mathrm{L}^{-1}\right)$ harvested 2006 and stored for five and eight months

\begin{tabular}{|c|c|c|c|c|c|c|c|c|c|c|c|c|c|c|c|c|c|c|c|c|}
\hline \multirow{2}{*}{ Period } & \multirow{2}{*}{\multicolumn{2}{|c|}{ Parameter }} & \multicolumn{18}{|c|}{ Specific gravity } \\
\hline & & & \multicolumn{3}{|c|}{$<1.055$} & \multicolumn{3}{|c|}{$1.056-1.065$} & \multicolumn{3}{|c|}{$1.066-1.075$} & \multicolumn{3}{|c|}{$1.076-1.085$} & \multicolumn{3}{|c|}{$1.086-1.095$} & \multicolumn{3}{|c|}{$>1.095$} \\
\hline \multirow{7}{*}{ Harvest } & Water concentration & $\mathrm{A}$ & & & & 847.20 & \pm 39.5 & $\mathrm{a}$ & 816.13 & \pm 36.9 & $\mathrm{ab}$ & 802.43 & \pm 56.9 & $\mathrm{~b}$ & 785.82 & \pm 32.6 & $\mathrm{~b}$ & & & \\
\hline & Water potential & $\mathrm{A}$ & & & & -0.73 & \pm 0.1 & $\mathrm{a}$ & -0.69 & \pm 0.1 & $\mathrm{a}$ & -0.73 & \pm 0.1 & $\mathrm{a}$ & -0.61 & \pm 0.1 & $\mathrm{a}$ & & & \\
\hline & Osmolality & $\mathrm{C}$ & & & & 471.08 & \pm 22.6 & $\mathrm{a}$ & 422.27 & \pm 24.0 & $\mathrm{a}$ & 409.24 & \pm 24.1 & $\mathrm{a}$ & 352.35 & \pm 7.7 & $\mathrm{a}$ & & & \\
\hline & Osmotic potential & $\mathrm{A}$ & & & & -1.15 & \pm 0.1 & $\mathrm{a}$ & -1.03 & \pm 0.1 & $\mathrm{a}$ & -1.00 & \pm 0.1 & $\mathrm{a}$ & -0.86 & \pm 0.0 & $\mathrm{a}$ & & & \\
\hline & Modulus of elasticity & $\mathrm{B}$ & & & & 3.08 & \pm 1.5 & $\mathrm{a}$ & 2.62 & \pm 1.1 & $\mathrm{a}$ & 2.21 & \pm 0.9 & $\mathrm{a}$ & 2.98 & \pm 1.0 & $\mathrm{a}$ & & & \\
\hline & Pressure potential & $\mathrm{AB}$ & & & & 0.43 & \pm 0.1 & $\mathrm{a}$ & 0.34 & \pm 0.1 & $\mathrm{a}$ & 0.27 & \pm 0.1 & $\mathrm{a}$ & 0.25 & \pm 0.1 & $\mathrm{a}$ & & & \\
\hline & BSI & $\mathrm{B}$ & & & & 10.50 & \pm 2.8 & $\mathrm{c}$ & 18.58 & \pm 4.0 & $\mathrm{c}$ & 37.00 & \pm 4.5 & $\mathrm{~b}$ & 89.68 & \pm 3.1 & $\mathrm{a}$ & & & \\
\hline \multirow{7}{*}{$\begin{array}{l}\text { Five months } \\
\text { stored }\end{array}$} & Water concentration & A & 892.12 & \pm 26.5 & $\mathrm{a}$ & 852.44 & \pm 22.7 & $\mathrm{a}$ & 829.03 & \pm 34.4 & $\mathrm{a}$ & 804.19 & \pm 39.0 & $\mathrm{a}$ & 790.22 & \pm 36.2 & $\mathrm{~b}$ & 761.71 & \pm 17.2 & $\mathrm{c}$ \\
\hline & Water potential & $\mathrm{B}$ & -0.88 & \pm 0.0 & $\mathrm{a}$ & -0.90 & \pm 0.0 & $a b$ & -0.91 & \pm 0.1 & $\mathrm{ab}$ & -0.99 & \pm 0.1 & $\mathrm{~b}$ & -0.97 & \pm 0.1 & $\mathrm{~b}$ & -0.87 & \pm 0.1 & $\mathrm{a}$ \\
\hline & Osmolality & $\mathrm{B}$ & 427.35 & \pm 3.2 & $\mathrm{ab}$ & 467.61 & \pm 7.6 & $\mathrm{~b}$ & 503.42 & \pm 13.8 & $\mathrm{ab}$ & 511.26 & \pm 7.9 & $a b$ & 564.51 & \pm 26.8 & $\mathrm{a}$ & 469.50 & \pm 4.8 & $\mathrm{~b}$ \\
\hline & Osmotic potential & $\mathrm{B}$ & -1.04 & \pm 0.0 & $\mathrm{ab}$ & -1.14 & \pm 0.0 & $\mathrm{a}$ & -1.23 & \pm 0.0 & $\mathrm{a}$ & -1.25 & \pm 0.0 & $a b$ & -1.38 & \pm 0.1 & $\mathrm{~b}$ & -1.15 & \pm 0.0 & $a b$ \\
\hline & Modulus of elasticity & $\mathrm{B}$ & 3.30 & \pm 0.1 & $\mathrm{a}$ & 4.11 & \pm 1.5 & $\mathrm{a}$ & 4.58 & \pm 1.9 & $\mathrm{a}$ & 3.73 & \pm 1.7 & $\mathrm{a}$ & 5.70 & \pm 3.1 & $\mathrm{a}$ & 3.23 & \pm 1.2 & $\mathrm{a}$ \\
\hline & Pressure potential & $\mathrm{B}$ & 0.16 & \pm 0.0 & $\mathrm{a}$ & 0.24 & \pm 0.0 & $\mathrm{a}$ & 0.32 & \pm 0.1 & $\mathrm{a}$ & 0.26 & \pm 0.1 & $\mathrm{a}$ & 0.41 & \pm 0.1 & $\mathrm{a}$ & 0.28 & \pm 0.1 & $\mathrm{a}$ \\
\hline & BSI & $\mathrm{A}$ & & & & 21.45 & \pm 3.3 & $\mathrm{c}$ & 31.14 & \pm 3.3 & $\mathrm{~b}$ & 45.20 & \pm 4.1 & $\mathrm{~b}$ & 77.00 & \pm 5.2 & $\mathrm{a}$ & & & \\
\hline \multirow{7}{*}{$\begin{array}{l}\text { Eight months } \\
\text { stored }\end{array}$} & Water concentration & A & 865.29 & \pm 28.2 & $\mathrm{a}$ & 833.02 & \pm 31.6 & $\mathrm{a}$ & 807.59 & \pm 29.5 & $\mathrm{ab}$ & 787.99 & \pm 23.6 & $\mathrm{bc}$ & 761.59 & \pm 41.0 & $\mathrm{c}$ & 766.40 & \pm 24.1 & $\mathrm{bc}$ \\
\hline & Water potential & $\mathrm{B}$ & -1.07 & \pm 0.1 & $\mathrm{ab}$ & -0.92 & \pm 0.1 & $\mathrm{a}$ & -0.91 & \pm 0.1 & $\mathrm{a}$ & -1.01 & \pm 0.1 & $a b$ & -1.11 & \pm 0.2 & $\mathrm{~b}$ & -1.04 & \pm 0.1 & $a b$ \\
\hline & Osmolality & $\mathrm{A}$ & 530.10 & \pm 44.6 & $\mathrm{a}$ & 529.50 & \pm 39.8 & $\mathrm{a}$ & 530.51 & \pm 34.6 & $\mathrm{a}$ & 571.50 & \pm 72.1 & $\mathrm{a}$ & 586.57 & \pm 57.1 & $\mathrm{a}$ & 589.65 & \pm 53.4 & $\mathrm{a}$ \\
\hline & Osmotic potential & $\mathrm{C}$ & -1.30 & \pm 0.1 & $\mathrm{a}$ & -1.29 & \pm 0.1 & $\mathrm{a}$ & -1.30 & \pm 0.1 & $\mathrm{a}$ & -1.40 & \pm 0.2 & $\mathrm{a}$ & -1.43 & \pm 0.2 & $\mathrm{a}$ & -1.44 & \pm 0.1 & $\mathrm{a}$ \\
\hline & Modulus of elasticity & $\mathrm{A}$ & 4.45 & \pm 4.0 & $\mathrm{a}$ & 5.98 & \pm 2.9 & $\mathrm{a}$ & 6.41 & \pm 3.8 & $\mathrm{a}$ & 6.43 & \pm 1.9 & $\mathrm{a}$ & 5.54 & \pm 2.4 & $\mathrm{a}$ & 6.18 & \pm 4.9 & $\mathrm{a}$ \\
\hline & Pressure potential & A & 0.23 & \pm 0.1 & $\mathrm{a}$ & 0.38 & \pm 0.1 & $\mathrm{a}$ & 0.39 & \pm 0.1 & $\mathrm{a}$ & 0.39 & \pm 0.1 & $\mathrm{a}$ & 0.32 & \pm 0.1 & $\mathrm{a}$ & 0.40 & \pm 0.2 & $\mathrm{a}$ \\
\hline & BSI & $\mathrm{B}$ & & & & 4.33 & \pm 1.7 & $\mathrm{c}$ & 14.67 & \pm 2.0 & $\mathrm{~b}$ & 27.88 & \pm 3.0 & $\mathrm{~b}$ & 42.12 & \pm 3.1 & $\mathrm{~b}$ & 55.00 & \pm 3.4 & $\mathrm{a}$ \\
\hline
\end{tabular}

${ }_{\mathrm{A}, \mathrm{B}, \mathrm{C}}$ different upper case letters indicate significant differences between test periods within single parameters by Tukey's test ( $\left.\mathrm{p} \leq 0.05\right)$, irrespective of specific gravity

a.b.c different lower case letters indicate significant differences of tubers by Tukey's test $(\mathrm{p} \leq 0.05)$ within single parameters and tests periods 
Table 3.2 Water concentration ( $\mathrm{g} \mathrm{kg}^{-1} \mathrm{FM}$ ), water potential (MPa), osmolality ( $\left.\mathrm{mmol} \mathrm{kg}^{-1}\right)$, osmotic potential (MPa), modulus of elasticity (MPa), pressure potential (MPa) and BSI (\%) of tubers differing in their specific gravity $\left(\mathrm{kg} \mathrm{L}^{-1}\right)$ harvested 2007 and stored for five and eight months

\begin{tabular}{|c|c|c|c|c|c|c|c|c|c|c|c|c|c|c|c|c|c|c|c|c|}
\hline \multirow{2}{*}{ Period } & \multirow{2}{*}{\multicolumn{2}{|c|}{ Parameter }} & \multicolumn{18}{|c|}{ Specific gravity } \\
\hline & & & \multicolumn{3}{|c|}{$<1.055$} & \multicolumn{3}{|c|}{$1.056-1.065$} & \multicolumn{3}{|c|}{$1.066-1.075$} & \multicolumn{3}{|c|}{$1.076-1.085$} & \multicolumn{3}{|c|}{$1.086-1.095$} & \multicolumn{3}{|c|}{$>1.095$} \\
\hline \multirow{6}{*}{ Harvest } & Water concentration & A & 876.77 & \pm 28.2 & $\mathrm{a}$ & 838.48 & \pm 32.2 & $a b$ & 827.36 & \pm 60.6 & $\mathrm{ab}$ & 803.27 & \pm 36.2 & $\mathrm{~b}$ & 774.77 & \pm 25.9 & $\mathrm{~b}$ & 757.13 & \pm 38.4 & $\mathrm{~b}$ \\
\hline & Water potential & A & -0.72 & \pm 0.1 & $\mathrm{a}$ & -0.72 & \pm 0.1 & $\mathrm{a}$ & -0.71 & \pm 0.0 & $\mathrm{a}$ & -0.73 & \pm 0.0 & $\mathrm{a}$ & -0.72 & \pm 0.0 & $\mathrm{a}$ & -0.74 & \pm 0.0 & $\mathrm{a}$ \\
\hline & Osmolarlity & $\mathrm{C}$ & 394.65 & \pm 26.7 & a & 391.63 & \pm 13.8 & $\mathrm{a}$ & 398.78 & \pm 13.9 & $\mathrm{a}$ & 404.21 & \pm 16.8 & $\mathrm{a}$ & 403.05 & \pm 8.8 & $\mathrm{a}$ & 390.30 & \pm 11.1 & $\mathrm{a}$ \\
\hline & Osmotic potential & A & -0.96 & \pm 0.1 & $\mathrm{a}$ & -0.96 & \pm 0.0 & $\mathrm{a}$ & -0.97 & \pm 0.0 & $\mathrm{a}$ & -0.99 & \pm 0.0 & $\mathrm{a}$ & -0.99 & \pm 0.0 & $\mathrm{a}$ & -0.95 & \pm 0.0 & a \\
\hline & Pressure potential & $\mathrm{B}$ & 0.25 & \pm 0.1 & $a b$ & 0.24 & \pm 0.0 & $a b$ & 0.27 & \pm 0.0 & $\mathrm{a}$ & 0.26 & \pm 0.0 & $\mathrm{ab}$ & 0.27 & \pm 0.0 & $\mathrm{a}$ & 0.21 & \pm 0.0 & $\mathrm{~b}$ \\
\hline & BSI & A & 4.50 & \pm 1.6 & $\mathrm{c}$ & 35.03 & \pm 3.9 & $\mathrm{c}$ & 50.43 & \pm 4.0 & $\mathrm{~b}$ & 67.53 & \pm 3.8 & $\mathrm{ab}$ & 98.77 & \pm 1.2 & $\mathrm{a}$ & & & \\
\hline \multirow{7}{*}{$\begin{array}{l}\text { Five months } \\
\text { stored }\end{array}$} & Water concentration & A & 863.10 & \pm 14.8 & $\mathrm{a}$ & 846.59 & \pm 27.1 & $a b$ & 831.15 & \pm 26.0 & $\mathrm{ab}$ & 817.08 & \pm 21.9 & $\mathrm{~b}$ & 785.42 & \pm 17.6 & $\mathrm{c}$ & 765.25 & \pm 18.5 & $\mathrm{c}$ \\
\hline & Water potential & $\mathrm{B}$ & -0.80 & \pm 0.0 & $\mathrm{a}$ & -0.81 & \pm 0.0 & $\mathrm{a}$ & -0.81 & \pm 0.0 & $\mathrm{a}$ & -0.82 & \pm 0.1 & $\mathrm{a}$ & -0.83 & \pm 0.0 & a & -0.81 & \pm 0.0 & $\mathrm{a}$ \\
\hline & Osmolarlity & $\mathrm{B}$ & 450.00 & \pm 7.1 & $\mathrm{a}$ & 451.85 & \pm 17.5 & $\mathrm{a}$ & 456.08 & \pm 18.8 & $\mathrm{a}$ & 450.75 & \pm 19.5 & $\mathrm{a}$ & 461.80 & \pm 12.2 & $\mathrm{a}$ & 453.60 & \pm 16.5 & $\mathrm{a}$ \\
\hline & Osmotic potential & $\mathrm{B}$ & -1.10 & \pm 0.0 & $\mathrm{a}$ & -1.10 & \pm 0.0 & $\mathrm{a}$ & -1.11 & \pm 0.1 & $\mathrm{a}$ & -1.10 & \pm 0.1 & $\mathrm{a}$ & -1.13 & \pm 0.0 & $\mathrm{a}$ & -1.11 & \pm 0.0 & $\mathrm{a}$ \\
\hline & Modulus of elasticity & $\mathrm{B}$ & 2.08 & \pm 0.3 & $\mathrm{a}$ & 2.20 & \pm 0.4 & $\mathrm{a}$ & 2.40 & \pm 0.5 & a & 2.04 & \pm 0.4 & $\mathrm{a}$ & 2.11 & \pm 0.3 & $\mathrm{a}$ & 1.98 & \pm 0.4 & $\mathrm{a}$ \\
\hline & Pressure potential & A & 0.30 & \pm 0.0 & $\mathrm{a}$ & 0.29 & \pm 0.04 & $\mathrm{a}$ & 0.30 & \pm 0.0 & $\mathrm{a}$ & 0.29 & \pm 0.0 & $\mathrm{a}$ & 0.30 & \pm 0.0 & $\mathrm{a}$ & 0.30 & \pm 0.0 & $\mathrm{a}$ \\
\hline & BSI & $\mathrm{B}$ & & & & 31.67 & \pm 4.11 & $\mathrm{~d}$ & 25.33 & \pm 4.1 & $\mathrm{c}$ & 50.19 & \pm 4.6 & $\mathrm{~b}$ & 95.93 & \pm 0.6 & $\mathrm{a}$ & & & \\
\hline \multirow{7}{*}{$\begin{array}{l}\text { Eight months } \\
\text { stored }\end{array}$} & Water concentration & A & 827.96 & \pm 28.2 & $\mathrm{a}$ & 816.78 & \pm 25.8 & $a b$ & 800.90 & \pm 27.8 & $\mathrm{ab}$ & 793.26 & \pm 24.5 & $a b$ & 774.33 & \pm 20.9 & $a b$ & 746.83 & \pm 21.7 & $\mathrm{~b}$ \\
\hline & Water potential & $\mathrm{C}$ & -0.91 & \pm 0.1 & $\mathrm{a}$ & -0.91 & \pm 0.1 & $\mathrm{a}$ & -0.91 & \pm 0.1 & $\mathrm{a}$ & -0.88 & \pm 0.0 & $\mathrm{a}$ & -0.88 & \pm 0.1 & $\mathrm{a}$ & -0.95 & \pm 0.1 & $\mathrm{a}$ \\
\hline & Osmolarlity & A & 481.05 & \pm 31.9 & a & 478.39 & \pm 27.3 & a & 477.39 & \pm 27.3 & a & 472.89 & \pm 12.4 & $\mathrm{a}$ & 437.95 & \pm 38.6 & $\mathrm{a}$ & 463.28 & \pm 9.8 & $\mathrm{a}$ \\
\hline & Osmotic potential & $\mathrm{B}$ & -1.18 & \pm 0.1 & $\mathrm{a}$ & -1.17 & \pm 0.1 & $\mathrm{a}$ & -1.17 & \pm 0.0 & $\mathrm{a}$ & -1.16 & \pm 0.0 & $\mathrm{a}$ & -1.07 & \pm 0.1 & $\mathrm{a}$ & -1.13 & \pm 0.0 & $\mathrm{a}$ \\
\hline & Modulus of elasticity & $\mathrm{B}$ & 1.74 & \pm 0.2 & $\mathrm{a}$ & 2.23 & \pm 0.6 & $\mathrm{a}$ & 2.14 & \pm 0.5 & $\mathrm{a}$ & 2.43 & \pm 0.6 & $\mathrm{a}$ & 2.16 & \pm 0.3 & $\mathrm{a}$ & 1.68 & \pm 0.6 & $\mathrm{a}$ \\
\hline & Pressure potential & $\mathrm{B}$ & 0.26 & \pm 0.0 & a & 0.26 & \pm 0.1 & $\mathrm{a}$ & 0.26 & 0.05 & $\mathrm{a}$ & 0.28 & 0.06 & $\mathrm{a}$ & 0.19 & 0.08 & $\mathrm{a}$ & 0.18 & 0.07 & $\mathrm{a}$ \\
\hline & BSI & $\mathrm{C}$ & & & & 4.40 & \pm 2.46 & $\mathrm{c}$ & 2.49 & 1.37 & $\mathrm{c}$ & 23.21 & 5.06 & $\mathrm{~b}$ & 50.39 & 4.32 & $\mathrm{a}$ & & & \\
\hline
\end{tabular}

${ }_{\mathrm{A}, \mathrm{B}, \mathrm{C}}$ different upper case letters indicate significant differences between test periods within single parameters by Tukey's test (p $\left.\leq 0.05\right)$, irrespective of specific gravity

a.b.c different lower case letters indicate significant differences of tubers by Tukey's test $(\mathrm{p} \leq 0.05)$ within single parameters and tests periods 


\section{Discussion}

No significant tuber position-dependent differences in water potential, osmolality, osmotic potential, pressure potential or in the modulus of elasticity could be detected in the present study. In contrast, Bajema et al. (1998b) reported lower tissue elasticity and consequently a higher impact susceptibility for stem ends compared to bud ends of 'Russet Burbank' and 'Atlantic' tubers. Due to the fact that larger cells, thicker cell walls and interrupted starch packing of cells of the stem end enhanced their susceptibility to blackspot (Lærke, 2001), while affecting tissue water status (von Willert et al., 1995). Olsen et al. (2003/4) measured a relative lower turgor at stem end compared to the bud end of tubers of 'Shepody'. This relationship was not observed in the present study.

Blackspot susceptibility and water status of tubers after harvest. In both years 2006 and 2007 tubers with a specific gravity lower than $1.095 \mathrm{~kg} \mathrm{~L}^{-1}$ showed significantly higher water potential after harvest, than after short-term or long-term storage. According to Suttle (2007) this result indicated that freshly harvested tubers were well hydrated despite, soil water was not longer available, while transpiration water losses of tubers were generally high a rapid decline of respiration might take place delaying metabolic processes (Galindo et al., 2004a; Suttle, 2007), whereby water was preserved. The concentration of stored macromolecules (starch and proteins) remained constant or decreased only slightly (annex, Tab. 10.50, 10.51) thus providing a sufficient resource of nutrients and carbohydrates for sprout development. This promptly declined metabolism in analysed tubers maintained their vitality.

The vitality of tubers is influenced by their pressure potential since its loss will inhibit sprouting (Galindo et al., 2004a). The pressure potentials found in harvested tubers ranged between 0.25 and $0.45 \mathrm{MPa}$ and are thus very similar to those reported for potatoes of 'Maries Piper' (0.36 MPa; Alvarez et al., 2000a), 'Katahdin' (0.36 MPa; Brusewitz et al., 1989), 'Russet Burbank' and 'Shepody' (0.26 MPa; Laza et al., 2001), and 'Dali' and 'Oleva' (0.43 MPa and 0.44 MPa, respectively; Lærke, 2001). This indicates only a minor difference between our measured pressure potentials and those reported in literature. Storey (2007) proposed that pressure potential may vary between different potato cultivars differing in their specific gravity. Tuber pressure potential was highly affected by the state of plant development or senescence, defoliation and the activity of water uptake by the root system (Storey 2007). However, analysed tubers (Fig. 3.1) were grown at similar conditions in the same field and planted and harvested at once displaying similar pressure potentials. Furthermore the modulus of elasticity of well-watered and turgid, freshly harvested tubers did not differ significantly (Tab. 3.1, 3.2). This corresponds to results given by Alvarez and Canet (2002) who found a generally reduced variability of tissue elasticity (Young's modulus) measurements at high pressure potentials.

Nevertheless, a decreasing pressure potential may further increase blackspot susceptibility as reported by Lærke (2001). Tubers harvested in 2007 revealed significantly lower pres- 
sure potentials and higher blackspot susceptibility compared to tubers harvested in 2006, despite the fact that temperatures during harvest and tuber water concentration were similar in 2006 and 2007. Presented results correspond to findings of Herppich et al. (2004) indicating that, at a given temperature, carrots show a lower toughness at a lower pressure potential.

When water uptake was restricted and increasing specific gravity indicated a higher dry matter concentration due to a higher amount of starch granules accompanied with a lower water concentration of tissues the tuber tissues became more elastic due to the lower pressure potential and the decreased elastic modulus. The susceptibility to blackspot might increase because of a reduced cell wall tension and/or a higher concentration of starch granules as it was discussed by Bajema et al. (1998a) and Scanlon et al. (1998).

Blackspot susceptibility and water status of stored tubers. Processing industry demands a permanent supply of potato tubers throughout the year. Therefore potato storage is essential in which tubers may undergo pronounced physiological changes (Laza et al., 2001; Scanlon et al., 1996). Despite the fact that these physiological changes of potato tubers have been widely studied (Alvarez and Canet, 2002; Baritelle and Hyde, 2001; Lærke, 2001; Scanlon et al., 1996) information are still incomplete in which way low temperatures might influence storage physiology.

In both years, tubers obviously accumulated osmotically active solutes which significantly decreased the water potential without decreasing pressure potential during the entire storage period. Within this process of osmotic adjustment (Galindo et al., 2004a) tissue sap osmolality increased in average by more than $100 \mathrm{mmol} \mathrm{kg}^{-1}$ due to the hydrolysis of starch to sucrose (Espen et al., 1999). This resulted in a decline in osmotic potential of 0.2 $\mathrm{MPa}$ and more. Osmotic adjustment may be a direct response to cold temperatures helping to reduce the freezing temperature of the tissue sap, thus, increasing tuber frost resistance. As a result of this process tubers may also stabilize or even increase their pressure potential despite a decreasing water potential (Galindo et al., 2004b). Remained pressure potentials during the entire storage indicated a steady membrane integrity, which was also not influenced by osmotic adjustment during storage corresponding to findings from Olsen et al. (2003/4). The absolute water concentration did not change during storage, while a decreasing water concentration would have indicated enhanced respiration usually accompanied with sprouting or senescence (Suttle, 2007 ), which was not observed in the present study. The tubers obviously maintained their vitality during storage as also described by Galindo et al. (2004b) and Heuer and Nadler (1998).

Blackspot susceptibility and tuber water status-synopsis. Observed variation in blackspot susceptibility at similar pressure potentials might be attributed to non-aqueous constituents of tissue. Both parameters are often reported to be positively correlated within potato tubers (Baritelle and Hyde, 2003; Peters et al., 2005; Wrigth et al., 2005). Throughout the whole shelf time of eight months blackspot significantly increased with the specific 
gravity. Specific gravity is generally assumed to be a cultivar characteristic (Laboski and Kelling, 2007; Neumann, 1995) and reflects the dry matter concentration of potato tubers (Haase 2003/4). A higher dry matter concentration may result from a higher amount of cell wall components, cytoplasmatic components or in case of potato tubers from a large size or a high number of starch granules. This reportedly increases the susceptibility to mechanical deformation of membranes as a direct effect of impact (Lærke, 2001; Olsen, 2003/4; Storey, 2007), regardless of tissue turgidity.

According to Scanlon et al. (1998) and Bajema et al. (1998a) it may be assumed that a fully turgid vacuole presses protoplasm and starch granules against the cell walls more than in flaccid cells. Compared to flaccid cells this may increase the shock wave speed after impact. The cell walls could compensate the acceleration of shock wave due to the chemical interactions between the various cell wall components or transferred the impact energy into the parenchyma.

Contrary, in relaxed cells the vacuoles did not perform such a strong pressure and so starch granules and protoplasm were not constricted. If the parenchyma was impacted, the starch granules were highly variable to the parenchyma and extended the cell membrane (Galindo et al., 2004b; Alvarez et al., 2000b) or dissipated the impact energy due to friction (Scanlon et al., 1998). Therefore, in flaccid cells the specific gravity of cells influences impact susceptibility. These findings are in agreement with results of Scanlon et al. (1998), who observed a positive relationship between specific gravity and loss modulus, a component of the modulus of elasticity.

A directly negative correlation between water concentration and blackspot was found in tubers with specific gravities above $1.095 \mathrm{~kg} \mathrm{~L}^{-1}$ during the entire period of investigation. The decreasing water concentration reduced stress threshold and enhanced impact susceptibility of potato tubers. Similar results were reported for potato tubers (Alvarez et al., 2000a), carrot roots (Herppich et al., 2004) and apples (Alvarez et al., 2000b).

\section{Conclusion}

From the present results we conclude that blackspot susceptibility of potato tissue is primary associated with the starch concentration of cells and furthermore with the impact susceptibility of the cell membrane of the cortical parenchyma. Presented data showed that increasing impact thresholds and therefore lower blackspot susceptibilities were supported by turgid cells achieved through a balanced water concentration of tubers during growth and storage periods irrespective of cultivars.

\section{References}

Alvarez, M.D., Canet, W., 2002. Effect of osmotic adjustment on the rheology of potato tissue. The use of discriminant analysis for interpretation. Eur. Food Res. Technol. 214, 83-90. 
Alvarez, M.D., Saunders, D.E.J., Vincent, J.F.V., 2000a. Effects of turgor pressure on the cutting energy of stored potato tissue. Eur. Food Res. Technol. 210, 331-339.

Alvarez, M.D., Saunders, D.E.J., Vincent, J.F.V., 2000b. Fracture properties of stored fresh and osmotically manipulated apple tissue. Eur. Food Res. Technol. 211, 284-290.

Bajema, R.W., Hyde, G.M., Baritelle, A.L., 1998a. Turgor and temperature effects on dynamic failure properties of potato tuber tissue. T. ASAE. 41, 741-746.

Bajema, R.W., Hyde, G.M., Baritelle, A.L., 1998b. Temperature and strain effects on the dynamic failure properties of potato tuber tissue. T. ASAE. 41, 733-740.

Baritelle, A., Hyde, G.H., 2003. Specific gravity and cultivar effects on potato tuber impact sensitivity. Postharvest Biol. Technol. 29, 279-286.

Baritelle, A.L., Hyde, G.H., 2001. Commodity conditions to reduce impact bruising. Postharvest Biol. Technol. 21, 331-339.

Brusewitz, G.H., Pitt, R.E., Gao, Q., 1989. Effects of storage with time and static preloading on the rheology of potato tissue. J. Texture Stud. 20, 267-284.

Corsini, D., Stark, J., Thornton, M., 1999. Factors contributing to the blackspot bruise potential of Idaho Potato fields. Amer. J. of Potato Res. 76, 221-226.

Espen, L., Morgutti, S., Abruzzese, A., Negrini, N., Rivetta, A., Quattrini, M.M., Cocucci, M., Cocucci, S.M., 1999. Changes in the potato (Solanum tuberosum L.) tuber at the onset of dormancy and during storage at $23{ }^{\circ} \mathrm{C}$ and $3{ }^{\circ} \mathrm{C}$. I. Biochemical and physiological parameters. Potato Research 42, 189-201.

Galindo, F.G., Herppich, W.B., Gekas, V., Sjöholm, I., 2004b. Factors affecting quality and postharvest properties of vegetables: Integration of water relations and metabolism. Crit. Rev. Food Sci. Nutr. 44, 139-154.

Galindo, F.G., Vaughan, D., Herppich, W.B., Smallwood, M., Sommarin, M., Gekas, V., Sjöholm, I., 2004a. Influence of cold accumulation on the mechanical strength of carrot (Daucus carota L.) tissue. Europ. J. Hort. Sci. 69, 1-6.

Günel, E., Karadogan, T., 1998. Effect of irrigation applied at growth stages and length of irrigation on quality characters of potato tubers. Potato Research. 41, 9-19.

Haase, N.U., 2003/4. Estimation of dry matter and starch concentration in potatoes by determination of under-water weight and near infrared spectroscopy. Potato Research. 46, 117-127.

Herppich, W.B., Herold, B., Geyer, M., Gomez, F., 2004. Effects of temperature and water relations on carrots and radish tuber texture. J. Appl. Bot. 78, 11-17.

Herppich, W.B., Mempel, H., Geyer, M., 1999. Effects of postharvest mechanical and climatic stress on carrot tissue water relations. Postharvest Biol. Technol. 16, 43-49. 
Heuer, B., Nadler, A., 1998. Physiological response of potato plants to soil salinity and water deficit. Plant Sci. 137, 43-51.

Kolbe, H., Stephan-Beckmann, S., 1997. Development, growth and chemical composition of the potato crop (Solanum tuberosum L.). II. Tuber and whole plant. Potato Research. 40, 135-153.

Laboski, C.A.M., Kelling, K.A., 2007. Influence of fertilizer management and soil fertility on tuber specific gravity: a review. Amer. J. of Potato Res. 84, 3-290.

Lærke, P.E., 2001. Blackspot bruise in potato tubers. Ph. D. thesis, Department of Plant Biology, The Royal Veterinary and Agricultural University, Denmark.

Lærke, P.E., Christiansen, J., Veierskov, B., 2002. Colour of blackspot bruises in potato tubers during growth and storage compared to their discolouration potential. Postharvest Biol. Technol. 26, 99-111.

Landahl, S., Herppich, W.B., Herold, B., Geyer, M., 2004. A comprehensive evaluation of the interaction between produce elasticity and water status. Europ. J. Hort. Sci. 69 (6), $1-6$.

Landrigan, M., Morris, S.C., Eamus, D., McGlasson, W.B., 1996. Postharvest water relationships and tissue browning of rambutan fruit. Sci. Hortic. 66, 201-208.

Laza, M., Scanlon, M.G., Mazza, G., 2001. The effect of tuber pre-heating temperature and storage time on the mechanical properties of potatoes. Food Res. Int. 34, 659-667.

Lulai, E.C., 2007. Skin-set, wound healing, and related defects. In: Vreugdenhil, D. (Ed), Potato biology and biotechnology. Advances and perspectives. 1. Elsevier, Amsterdam, pp. 471-496.

Neumann, P.M., 1995. The roll of the cell wall adjustment in plant resistance to water deficits. Crop Sci. 35, 1258-1266.

Olsen, N., Thornton, R.E., Baritelle, A., Hyde, G. 2003/4. The influence of storage conditions on physical and physiological characteristics of Shepody potatoes. Potato Res. 46, 95-103.

Pawelzik, E., Delgado, E., 1999. Wirkung von Trockenstress auf die Verfärbungsneigung von Kartoffelknollen. Kartoffelbau 50, 358-360.

Peters, R., Haase, N.U., Pawelzik, E., 2005. Black spot susceptibility of potato tubers in relation to specific gravity. Abstracts of the $16^{\text {th }}$ Triennial Conference of the EAPR. Bilbao 17.-22. July 2005, pp. 916-918.

Rasool, A.-Z., Mojtaba, F., Davood, H.-P., 2007. Sequential path analysis of yield components in potato. Potato Res. 49, 273-279.

Scanlon, M.G., Day, A.J., Povey, M.J.W., 1998. Shear stiffness and density in potato parenchyma. Int. J. Food Sci. Tech. 33, 461-464. 
Scanlon, M.G., Pang, C.H., Biliaderis, C.G., 1996. The effect of osmotic adjustment on the mechanical properties of potato parenchyma. Food Res. Int. 29, 481-488.

Schmitz, G.E., Sullivan, M.L., Hatfield, R.D., 2008. Three polyphenol oxidases from red clover (Trifolium pratense) differ in enzymatic activities and activation properties. J. Agric. Food Chem. 56, 272-280.

Storey, M., 2007. The harvested crop. In: Vreugdenhil D. (Ed), Potato biology and biotechnology. Advances and perspectives. 1. Elsevier, Amsterdam, pp. 441-466.

Suttle, J.C., 2007. Dormancy and Sprouting. In: Vreugdenhil D. (Ed), Potato biology and biotechnology. Advances and perspectives. 1. Elsevier, Amsterdam, pp. 287-305.

von Willert, D.J., Matyssek, R., Herppich, W.B., 1995. Experimentelle Pflanzenökologie. Grundlagen und Anwendungen. Thieme-Verlag, Stuttgart.

Wright, P.J., Triggs, C.M., Anderson, J.A.D., 2005. Effects of specific gravity and cultivar on susceptibility of potato (Solanum tuberosum) tubers to blackspot bruising and bacterial soft rot. New Zeal. J. Crop Hort. Sci. 33, 353-361. 


\title{
4. Nutrient concentrations in potato (Solanum tuberosum) tubers in comparison with their different susceptibility towards blackspot
}

\begin{abstract}
The blackspot index (BSI) was determined in potato tubers harvested at different stages of haulm maturity and after storing tubers for several months. Pre-harvested tubers had a lower BSI compared to tubers harvested two weeks later (main harvest) whereas the BSI of stored tubers varied from year to year. The BSI increased when cultivar specific gravity was relative high. However, the concentrations of $\mathrm{K}, \mathrm{P}, \mathrm{Mg}, \mathrm{Ca}, \mathrm{Fe}, \mathrm{B}$ and Se were not related to the BSI of tubers. Irrigation to attain optimum soil moisture conditions during periods of vegetation enhanced nutrient sufficiency and reduced the potential damage inflicted on tubers. Nutrient uptake was nearly complete when the majority of tuber growth ends approximately 150 days after planting. Although extreme nutrient deficiency was not observed nutrient concentrations of tubers particularly depended on nutritional interactions influenced the BSI also.
\end{abstract}

Keywords: specific gravity, maturity, storage, fertilization, potassium, phosphorus, magnesium, calcium, iron, boron, selenium

\section{Introduction}

Potato tuber are rich in parenchymatous tissue including water as a predominant component and shows a lack of specialized thickened tissue (Peterson et al. 1985). This characteristic determine tubers susceptibility towards various forms of external and internal damage that occur after impact caused during commercial production, harvest and/or post-harvest handling (Thornton and Timm 1990). In contrast to external damage, internal damage is not visible before cutting or peeling the tuber. The symptoms of internal damage may or may not include visible tissue fracture. If there is no visible fracture, damage sometimes includes the development of floury-white regions in clear contrast to the cream background of undamaged tissue finally resulting in the formation of grey and black coloured pigment melanin. This symptom is usually referred to as internal bruising particularly termed blackspot (McGarry et al. 1996). A major factor on the intensity of blackspot is the speed, distance and type of impact as well as the genetic and environmental diversity. In general tubers of a given cultivar or among cultivars subjected to a known impact do not express the same degree of blackspot. This suggested that there is at least one component influencing blackspot susceptibility among tubers (Thornton and Timm 1990). Dry matter has a major influence on blackspot susceptibility, whereas dry matter primary depends on to the starch concentration of tubers (Wulkow et al. in preparation) and, therefore, it is highly variable. Even the alternation of the water concentration influences blackspot susceptibility (Thornton and Timm 1990). Also tuber maturity is a key factor because increasing maturity modifies the blackspot susceptibility (Corsini et al. 1999) as well as nutrient concentrations (Putz 1998). Besides C, H and O, potato plants require normally 14 essential nutrients 
namely N, P, K, Ca, Mg, S, Cl, Fe, Mn, B, Zn, Cu, Mo, Ni (White et al. 2007) that should be supplied from the soil solution and fertilizers (Westermann 2005). Plant nutrition including fertilizer management affects tuber quality (Putz 1998) and therefore tuber susceptibility towards blackspot (White et al. 2007). A large number of studies nominate $\mathrm{K}$ as one element influencing blackspot susceptibility of potato tubers (Workman and Holm 1984; Thornton and Timm 1990; McNabnay et al.1999; Lampe 2007). But there is some evidence that also other nutrients might influence blackspot susceptibility due to their function in plant cells. In this study seven nutrients were selected particularly from the pool of 14 essential nutrients to investigate their influence on blackspot susceptibility of potato tubers originated from different cultivars at different stages of haulm maturity. There is some evidence that blackspot susceptibility may be related to the cell membrane integrity (Dejmek and Miyawaki 2002; Scanlon et al. 1996), which is particularly affected by its concentration of P (Bergmann 1993; Bucher and Kossmann 2007), B (Mondi and Munshi 1993) and Ca (Sowokinos 2007; Tzeng et al. 1986; Davies 1998). In-season Ca fertilization to the tuber and stolon increases the $\mathrm{Ca}$ concentration in tubers but also reduces pathological and internal physiological disorders (Simmons and Kelling 1987) like internal brown spot (Tzeng et al. 1986; Bartz et al. 1992), brown centre (Davies 1998; Sowokinos 2007) and Hollow Heart (Sowokinos 2007). Also an inverse relationship between the Ca concentration of tubers and susceptibility of bacterial soft root (Tzeng et al. 1986) and blackspot was investigated (Lærke 2001). In conclusion, malnutrition related to P, B and/or Ca deficiency might affect intracellular robustness and further decompartmentalization may enhance the biochemical potential to synthesise melanin (Dean et al. 1993). Melanin formation is initiated by an enzyme-catalysed oxidation presupposing the presence of molecular oxygen (Edgell et al. 1998) or of superoxide radicals (Storey 2007). Nevertheless, both may act as reactive oxygen species (ROS) in plant cells (Blokhina et al. 2003) and their concentration may be limited by antioxidants (Lærke et al 2002). On the one hand potato tissue contains several enzymes scavenging ROS [several types of superoxide dismutase (SOD), catalase (CAT), peroxidase POX)] (Yahraus 1995; Blokhina et al. 2003; Leo et al. 2008) whose activities are enhanced by Se (Seppänen et al. 2003; Turakainen et al. 2006). Although Se is an essential nutrient for animals, bacteria and algae (Terry et al. 2000) but not for plants (McNeal and Balistrieri 1989) its concentration will be discussed subsequently. On the other hand ascorbic acid is one of the most common antioxidants in potato tubers among others (McNabnay et al. 1999; Pelegrini et al. 2003; Nzaramba et al. 2007). The concentration of organic acids especially ascorbic acid, dehydroascorbic acid and citric acid is high at an optimal supply of both elements $\mathrm{K}$ and Mg (Rogozińska et al. 2005). Also the concentration of malic acid is enhanced by an optimal supply of Fe (Bergmann 1993). The total concentration of organic acids might depress the blackspot susceptibility of potato tubers to some extent (Heinecke 2007). During blackspot formation malnutrition related to $\mathrm{Se}, \mathrm{K}, \mathrm{Mg}$ and/or Fe deficiency may promote further spontaneous oxidation and polymerisation of substrates catalysed by the enzyme PPO generating dopaquinone. Free tyrosine, 
cystein, chlorogenic acid and caffeic acid are PPO specific substrates present in potato tubers (Dean et al. 1993; Kirk et al. 2006). The amount of these substrates might be restricted or decreased at an optimal supply of K (McNabnay et al. 1999), B (Mondy and Munschi 1993), Se (Munshi et al. 1990) and Fe (Bergmann 1993). Despite the fact that a well balanced Fe concentration might mitigate blackspot susceptibility Fe also forms a complex with phenolic substances like chlorogenic acid esters during the cooking process of potato tubers causing after cooking darkening (Muneta and Kaisaki 1983; Griffiths and Bain 1997). Nevertheless, malnutrition associated with K, B, Se and/or Fe deficiency might increase substrate concentrations and, therefore, stimulate the continuing non-enzymatic polymerization reaction with nucleophilic amino acid residues of proteins and form the black nitrogen containing pigment melanin (Dean et al. 1993; Kirk et al. 2006). Depending on the compounds intermediates with varying colours occur (Dean et al. 1993; Kirk et al. 2006). With the present study we payed attention to the above described relationships between nutrient concentrations and blackspot susceptibility of potato tubers and investigated the effect of fertilizer on tuber quality when nutrients applied in amounts close to the requirement for producing optimal yield.

\section{Material and methods}

Material. Eight cultivars of table potatoes (Adretta, Afra, Gala, Granola, Lolita, Marabel, Nicola and Renate) were grown on sandy soils near Dethlingen, Germany, during two vegetation periods in 2006 and 2007. Conventional farming methods were practiced, including proper irrigation, adequate fertilization (urea and ammonium nitrate solution, diammonium phosphate as well as fertilizer combinations of $\mathrm{KCL}+\mathrm{Mg}$ and $\mathrm{K}_{2} \mathrm{SO}_{4}+\mathrm{Mg}$ were applied) and an integrated pest management (annex, Tab. 10.63, 10.64). To evaluate cultivar specific differences tubers of similar size from 40 to $50 \mathrm{~mm}$ and corresponding specific gravities were considered. Analyses were performed in tubers harvested two weeks before usual harvest time (pre-harvest) and in tubers reaped during ideal time of harvest as well as in tubers stored for five and eight months, respectively, at $4{ }^{\circ} \mathrm{C}$ and $95 \%$ relative humidity. Furthermore, the samples were divided into two parts. Tubers from the first part were used to determine their blackspot index (BSI). Those of the second part were sliced, shock-frozen in liquid nitrogen, lyophilized (Epsilon 2-40, Christ, Germany) and ground to $50 \mathrm{~mm}$ using a micro hammer mill (Culatti AG, Switzerland) to analyse the concentrations of Se, B, Fe, Ca, Mg, P and K, respectively.

Analyses. Blackspot index (BSI) of whole potato tubers of different specific gravities was determined according to the method proposed by the "Bundessortenamt" (BSA, Federal Plant Variety Office, Germany). The tubers were cooled down to 4 to $5^{\circ} \mathrm{C}$ and mechanical treated for $50 \mathrm{~s}$ in a vegetable washing machine with a rotating drum (Flott $18 \mathrm{~K}$, Flottwerk H. J. Dames GmbH \& Co. KG, Rotenburg a. d. F., Germany) with three replications. Each replication consisted of an amount of tubers $\left(\Sigma_{\text {tuber }}\right)$ adequate to a volume of $6 \mathrm{~L}$. Afterwards, treated tubers were stored at room temperature for 48 hours. For BSI determination 
they were cut into halves length-wise and a half tuber was evaluated visually using a scale with four scores of discolouration: No discolouration indicated the lack of any melanin development and therefore no blackspot susceptibility $\left(\Sigma_{1}\right)$. Discolouration of up to one quarter of length and less than $5 \mathrm{~mm}$ in depth denoted slight blackspot susceptibility $\left(\Sigma_{2}\right)$. Medium blackspot susceptibility corresponded to a discolouration of up to one quarter of length and more that $5 \mathrm{~mm}$ in depth or one half of the length and a maximum of $5 \mathrm{~mm}$ in depth $\left(\Sigma_{3}\right)$. Serious blackspot susceptibility was indicated by any discolouration stronger than that described before $\left(\Sigma_{4}\right)$. The blackspot susceptibility, expressed as the percentage of discoloured tubers was calculated as:

$$
B S I(\%)=\left(0.3 \sum_{2}+0.5 \sum_{3}+\sum_{4}\right) / \sum_{\text {tuber }} * 100
$$

BSI: blackspot index (\%)

$\Sigma:$ amount of analysed tubers

To determine the concentration of nutrients the ground samples were dissolved in $65 \%$ $\mathrm{HNO}_{3}$ (Roth, Germany) (Kuennen et al. 1982) during a thermic decomposition at $175^{\circ} \mathrm{C}$ for 12 hours, which was realized in pressurized Teflon vessels (Aysola et al. 1987). Dissolved samples with a final concentration of $40 \% \mathrm{HNO}_{3}$ were the basis for the following analyses. The measurement of $\mathrm{P}$ followed the official method of $\S 35 \mathrm{LMBG}$ L 06.00/9 (Wilhelm et al. 1993) using an UV-Vis spectral system (HP 8453, Germany) were the extinctions of samples were measured at $436 \mathrm{~nm}$ and the $\mathrm{P}$ concentration was determined according $\mathrm{KH}_{2} \mathrm{PO}_{4}$-calibration solutions with $\mathrm{P}$ concentrations between 0 to $14 \mathrm{mg} \mathrm{L}^{-1}$. The concentrations of $\mathrm{Se}, \mathrm{B}, \mathrm{Fe}, \mathrm{Ca}$ and $\mathrm{Mg}$ were detected with an inductively coupled plasma mass spectrometer (ICP MS) using established methods of the Geo-scientific Research Centre of the Faculty of Geoscience and Geography at the Georg-August-University of Goettingen. Therefore, multiple calibration solutions for $\mathrm{Se}, \mathrm{B}$ and $\mathrm{Fe}$ and for $\mathrm{Ca}$ and $\mathrm{Mg}$ were prepared. Characteristic isotopes of the minerals were chosen to avoid possible interferences with other isobaric masses and to obtain the highest peak to background ratio (Makishima et al. 1997; Müller et al. 2003). The concentration of K was measured using an atomic absorption spectrometer (Unicam Solaar M Series) applying appropriate dilutions (Westermann et al.1994; McNabnay et al. 1999).

Much of evidence showed nutrient concentrations of tubers as a function of dry matter (e.g. Locascio et al. 1992; Bergmann 1993; Bamberg et al. 1998; Putz 1998; Seppänen et al. 2003; Turakainen et al. 2004, 2006; Nitsch 2005; Rogozińska et al. 2005; Sulaiman 2005; Dilworth et al. 2007). In this study, nutrient concentrations are presented in tuber fresh weight because dry matter itself has a strong relationship towards blackspot susceptibility of potato tubers (Wulkow et al. in preparation) and might overrule nutritional interactions towards blackspot susceptibility.

Statistics. The obtained data were analysed with Sigma Stat statistical program (STAT32). Preliminary all data sets were tested for normal distribution and variance homogeneity $(p=$ 
0.05). Following analyses of variance were calculated for cultivar and specific gravity. Calculated means were compared by Tukey-test $(\mathrm{p} \leq 0.05)$. Correlation and multiple regression procedures between normally distributed quality parameters were performed using Pearson correlation coefficients. This technique does not determine significant differences between specific gravities within one cultivar but rather gives an overall indication of relative importance of both the genetic factor and tuber mass. Graphics were prepared with Sigma Plot 2001 (SPSS Inc. SigmaPlot for Windows).

\section{Results}

Weather conditions during both years of investigation 2006 and 2007 were not significantly different and did not depart from the average weather conditions metered for this typical potato-growing region (annex, Tab. 10.62). In both years the sunshine duration amounted approximately 1252 hours associated with a mean temperature of $15^{\circ} \mathrm{C}$ and a water supply of $564 \mathrm{~mm}$ particularly mediated through irrigation. Depending on the soil conditions the time of planting and harvesting differed slightly. In 2006 the vegetation period comprised 161 days contrary to 2007 where the period of vegetation was 168 days long. In both years 2006 and 2007 tubers were partially harvested two weeks before the main harvest (in the following named as pre-harvest) with the aim of investigate tubers at different stages of maturity.

Relationship between specific gravity, nutrient concentration and BSI. The relationship between tuber specific gravity, as determinant of tuber dry matter concentration (Haase 2003/2004) and BSI as well as the relationship between nutrient concentrations and BSI are illustrated in Figure 4.1. Moreover, absolute concentrations of nutrients in potato tubers did not always reflect imbalanced nutrient conditions (annex, Tab. 10.29 - 10.42), therefore the ratios between macro and micro nutrients were calculated according to Bergmann (1993) (Tab. 4.1). 

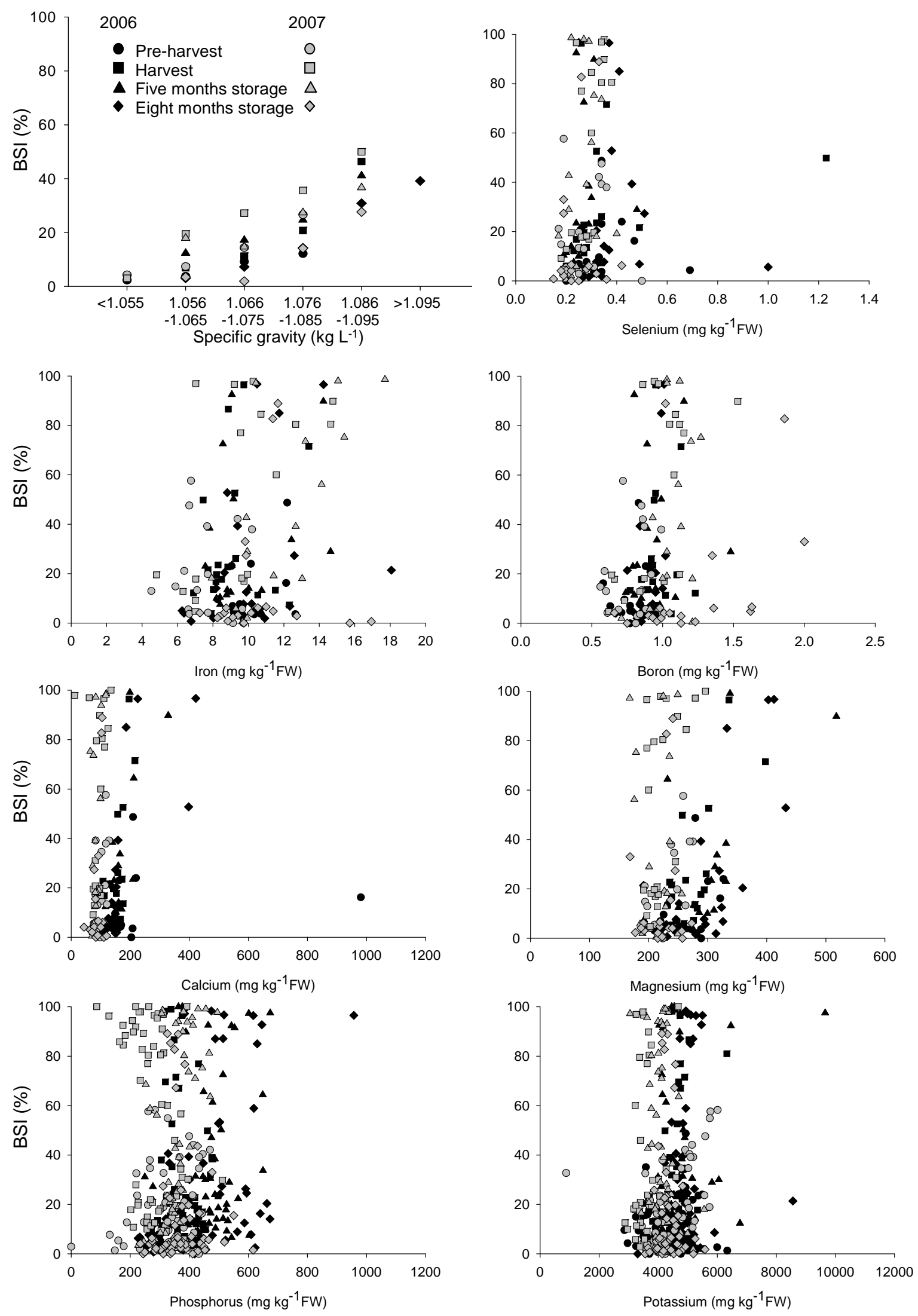

Figure 4.1 Relationship between tuber specific gravity $\left(\mathrm{kg} \mathrm{L}^{-1}\right)$ and BSI (\%) as well as tuber nutrient concentrations ( $\mathrm{mg} \mathrm{kg}^{-1} \mathrm{FW}$ ) and BSI (\%) determined in 2006 and 2007 after the pre-harvest, harvest and five and eight months of storage 
Table 4.1 The ratio of macro:micro nutrients of tubers harvested at different stages of haulm maturity in 2006 and 2007 and stored for five and eight months, respectively

\begin{tabular}{lllll}
\hline Period/ Parameter & K/B & Ca/B & P/B & P/Fe \\
\hline Pre-harvested 2006 & $5563.48 \pm 828.89$ & $261.35 \pm 214.15$ & n.a. & n.a. \\
Pre-harvested 2007 & $6274.85 \pm 1171.20$ & $145.03 \pm 30.63$ & $484.19 \pm 116.73$ & $51.71 \pm 17.06$ \\
Harvested 2006 & $4629.11 \pm 499.69$ & $161.64 \pm 23.77$ & $392.34 \pm 73.67$ & $42.52 \pm 9.85$ \\
Harvested 2007 & $4304.26 \pm 970.89$ & $99.65 \pm 18.94$ & $332.12 \pm 113.44$ & $34.46 \pm 11.79$ \\
$\begin{array}{l}\text { Harvested 2006 and } \\
\text { stored for five months }\end{array}$ & $5042.26 \pm 1082.88$ & $180.24 \pm 47.15$ & $511.57 \pm 95.64$ & $51.52 \pm 12.57$ \\
$\begin{array}{l}\text { Harvested 2007 and } \\
\text { stored for five months }\end{array}$ & $3827.71 \pm 360.83$ & $81.87 \pm 19.69$ & $323.78 \pm 39.92$ & $27.93 \pm 6.19$ \\
$\begin{array}{l}\text { Harvested 2006 and } \\
\text { stored for eight months }\end{array}$ & $5658.66 \pm 701.93$ & $221.26 \pm 25.86$ & $557.24 \pm 112.08$ & $51.02 \pm 10.56$ \\
$\begin{array}{l}\text { Harvested 2007 and } \\
\text { stored for five months }\end{array}$ & $4094.26 \pm 947.63$ & $85.56 \pm 18.9$ & $348.81 \pm 103.19$ & $35.15 \pm 9.98$ \\
\hline $\begin{array}{l}\text { n.a. not analysed } \\
\text { Results }\end{array}$ & & & & \\
\hline
\end{tabular}

Results show that tubers pre-harvested in 2006 generated specific gravities between $<1.055$ and $1.085 \mathrm{~kg} \mathrm{~L}^{-1}$ accompanied with an increasing BSI from 5 to $25 \%$, which was not significantly different. The nutrient concentrations were not related to BSI.

With increasing maturity of tubers specific gravity and BSI increased significantly. The specific gravity of harvested tubers ranged from $<1.055$ to $>1.095 \mathrm{~kg} \mathrm{~L}^{-1}$ and a significant increas in BSI was observed in tubers with specific gravities $>1.075 \mathrm{~kg} \mathrm{~L}^{-1}$. Also the concentrations of $\mathrm{Mg}$ and $\mathrm{B}$ increased during maturity. Contrary, the concentration of $\mathrm{Ca}$ declined significantly and the concentrations of K, P, Fe and Se did not changed compared to premature tubers. There was a significant relationship between the concentrations of $\mathrm{Ca}$ $(\mathrm{r}=0.68, \mathrm{p}<0.05)$ and $\mathrm{Mg}(\mathrm{r}=0.67, \mathrm{p}<0.05)$ (Fig. 4.1) as well as between the $\mathrm{Ca} / \mathrm{B}$ ratio $(\mathrm{r}=0.51, \mathrm{p}<0.05)($ Tab. 4.1) and BSI.

A storage period of five months induced a significantly enhanced BSI in all tubers. Again, there was a significant relationship between concentrations of $\mathrm{Ca}(\mathrm{r}=0.72, \mathrm{p}<0.05)$ and $\mathrm{Mg}$ $(\mathrm{r}=0.61, \mathrm{p}<0.05)($ Fig. 4.1) as well as between the $\mathrm{Ca} / \mathrm{B}$ ratio $(\mathrm{r}=0.52, \mathrm{p}<0.05)($ Tab. 4.1) and BSI.

The positive relationship between specific gravity and BSI was also found after long-term storage of eight months but the overall BSI decreased to the level of harvested tubers. A significant relationship between the concentrations of $\mathrm{Se}(\mathrm{r}=0.53, \mathrm{p}<0.05), \mathrm{Ca}(\mathrm{r}=0.73$, $\mathrm{p}<0.05), \mathrm{Mg}(\mathrm{r}=0.70, \mathrm{p}<0.05), \mathrm{P}(\mathrm{r}=0.52, \mathrm{p}<0.05)$ (Fig. 4.1) as well as between the nutrient ratios $\mathrm{P} / \mathrm{B}(\mathrm{r}=0.54, \mathrm{p}<0.05), \mathrm{Ca} / \mathrm{B}(\mathrm{r}=0.66, \mathrm{p}<0.05)$ (Tab. 4.1) and $\mathrm{BSI}$ was detected. 
Generally BSI of tubers harvested in 2006 was significantly lower compared to BSI of potato tubers harvested in 2007 (Fig. 4.1).

Tubers pre-harvested in 2007 again produced specific gravities between $<1.055$ to $1.085 \mathrm{~kg}$ $\mathrm{L}^{-1}$ and a BSI from $7 \%$ to $50 \%$, which was significantly different within specific gravity ranges. The nutrient concentrations were similar to tubers pre-harvest 2006. A significant relationship occurred between the concentrations of $\mathrm{Ca}(\mathrm{r}=0.51, \mathrm{p}<0.05)$ and $\mathrm{Mg}(\mathrm{r}=0.70$, $\mathrm{p}<0.05$ ) and BSI (Fig. 4.1).

Once more, specific gravity and BSI increased significantly with increasing maturity of tubers. Also the concentrations of Fe and B increased with tuber maturity. Contrary to that the concentrations of $\mathrm{K}$ and $\mathrm{Ca}$ declined while the concentrations of $\mathrm{P}, \mathrm{Mg}$ and $\mathrm{Se}$ stay constant. This time nutrient concentrations of $\mathrm{Ca}(\mathrm{r}=0.57, \mathrm{p}<0.05), \mathrm{Fe}(\mathrm{r}=0.68, \mathrm{p}<0.05), \mathrm{B}$ $(\mathrm{r}=0.59, \mathrm{p}<0.05)$ and $\mathrm{Se}(\mathrm{r}=0.55, \mathrm{p}<0.05)($ Fig. 4.1$)$ as well as the nutrient ratios of $\mathrm{K} / \mathrm{B}$ $(\mathrm{r}=-0.60, \mathrm{p}<0.05), \mathrm{P} / \mathrm{Fe}(\mathrm{r}=-0.55, \mathrm{p}<0.05)$ and $\mathrm{P} / \mathrm{B}(\mathrm{r}=-0.56, \mathrm{p}<0.05)(\mathrm{Tab} .4 .1)$ were related to the BSI.

Compared to the previous year during prolonged time of storage the BSI decreased significantly, whereas the positive relationship between specific gravity and BSI still existed. Again there was a significant relationship between the concentrations of $\mathrm{Ca}(\mathrm{r}=-0.56$, $\mathrm{p}<0.05), \mathrm{Fe}(\mathrm{r}=-0.70, \mathrm{p}<0.05)($ Fig. 4.1) and some nutrient ratios $\mathrm{K} / \mathrm{B}(\mathrm{r}=-0.57, \mathrm{p}<0.05)$ and $\mathrm{P} / \mathrm{Fe}(\mathrm{r}=-0.61, \mathrm{p}<0.05)$ (Tab. 4.1) and BSI after five months of storage but not after eight months of storage.

The nutritional status of the tubers. The above evaluated concentrations of single nutrients and nutrient ratios with respect to the BSI of tubers showed no regular relationship. The obtained nutrient concentrations of investigated tubers were compared with data from the literature using as a guiding principle for an adequate supply (Tab. 4.2). The absolute concentrations revealed no differences between analysed and referred nutrient concentrations, excepted for Se and Ca. However, due to the fact that Se is no essential nutrient for potato tubers (Bergmann 1993) information were rare about the function of Se in potato tubers and no data about the concentration of Se in tuber fresh weight were found. Contrary, $\mathrm{Ca}$ is a well studied nutrient and tubers of the presented investigation seemed to suffer from a higher $\mathrm{Ca}$ concentration compared to $\mathrm{Ca}$ concentration cited from other references. To ensure this observation the relative concentration of the nutrients were calculated from the sum of means of absolute data. Results indicated again a relative high $\mathrm{Ca}$ concentration compared to other nutrients (Tab. 4.3). 
Table 4.2 Concentration of selected nutrients $\left(\mathrm{mg} \mathrm{kg}^{-1} \mathrm{FW}\right)$ in potato tubers differing in their blackspot susceptibility irrespective of cultivar, vegetation period and time of storage in comparison to published data of these nutrients $\left(\mathrm{mg} \mathrm{kg}^{-1} \mathrm{FW}\right)$ in potato tubers irrespective of cultivar, vegetation period and time of storage

\begin{tabular}{l|l|l|l|l|l|l|l|l|l}
\hline Nutrient & \multicolumn{4}{|c|}{ Present study } & \multicolumn{5}{c}{ Literature data } \\
\hline & Minimum & Maximum & Mean & $\sigma_{\mathrm{x}}$ & Minimum & Maximum & Mean & $\sigma_{\mathrm{x}}$ & Reference \\
\hline $\mathrm{Se}$ & 0.17 & 0.68 & 0.30 & 0.17 & & & & & \\
$\mathrm{~B}$ & 0.45 & 1.86 & 0.94 & 0.20 & 0.2 & 1.1 & 0.65 & 0.64 & 1 \\
$\mathrm{Fe}$ & 3.83 & 21.94 & 9.58 & 2.49 & 3 & 22 & 7.48 & 7.2 & $1,4,5$ \\
$\mathrm{Ca}$ & 11.79 & 980.74 & 101.55 & 60.11 & 18 & 65 & 41.40 & 18.66 & $1,3,4$ \\
$\mathrm{Mg}$ & 102.98 & 517.84 & 145.29 & 89.60 & 124 & 278 & 213.2 & 57.59 & $1,3,4$ \\
$\mathrm{P}$ & 111.91 & 672.99 & 359.38 & 109.76 & 200 & 479 & 343.00 & 139.63 & 1,2 \\
$\mathrm{~K}$ & 2626.02 & 6326.07 & 4327.86 & 643.45 & 3300 & 5973 & 4753.25 & 1266.59 & $1,3,2$ \\
\hline
\end{tabular}

${ }^{\sigma x}$ standard deviation, ${ }^{1}$ Davies 1998, ${ }^{2}$ Dilworth et al. 2007, ${ }^{3}$ Lærke 2001, ${ }^{4}$ Putz 1998, ${ }^{5}$ Sikora 1999

Table 4.3 Relative concentration (\%) of selected nutrients in potato tubers differing in their blackspot susceptibility irrespective of cultivar, vegetation period and time of storage in comparison to the published data of relative concentration (\%) of these nutrients in potato tubers irrespective of cultivar, vegetation period and time of storage (sum of means of absolute data $=100 \%$ )

\begin{tabular}{lll}
\hline Nutrient & & \multicolumn{1}{c}{ Fresh weight $(\%)$} \\
\hline Se & Present study & Literature data calculated ${ }^{1}$ \\
\cline { 2 - 3 } $\mathrm{B}$ & 0.01 & n.a. \\
$\mathrm{Fe}$ & 0.02 & 0.01 \\
$\mathrm{Ca}$ & 0.19 & 0.14 \\
$\mathrm{Mg}$ & 2.05 & 0.77 \\
$\mathrm{P}$ & 2.94 & 3.98 \\
$\mathrm{~K}$ & 7.27 & 6.40 \\
\hline
\end{tabular}

${ }^{1}$ the absolute nutrient concentrations published by Putz 1998, Lærke 2001, Davies 1998, Dilworth et al. 2007 and Sikora 1999 were used for calculation; ${ }^{\text {n.a. }}$ not available

To study the influence of this high $\mathrm{Ca}$ concentration on other tuber nutrients the ratio between macro $(\mathrm{K}, \mathrm{Ca}$ and $\mathrm{P})$ and micro $(\mathrm{B}, \mathrm{Fe})$ nutrients was calculated according evaluations of Bergmann (1993). There were some ratios of macro:micro nutrient concentrations available, whereby the ratio 240:1 indicated an optimal supply, the ratio 1200:1 revealed micro nutrient deficiency and the ratio 7:1 micronutrient toxicity (Bergmann 1993). Applying these observations to the given results, an unbalanced $\mathrm{K} / \mathrm{B}$ ratio was indicated; irrespective of the high $\mathrm{Ca}$ concentration (Tab. 4.1). 


\section{Discussion}

Harvest date in comparison with BSI. Besides specific gravity also the length of the vegetation period influenced tubers blackspot susceptibility of tubers. Also Corsini (1999) and Thornton and Timm (1990) described that after a typically warm vegetative period specific gravity as well as the maturity of tubers harvested at different stages of haulm maturity were closely related to BSI. The time of interruption of the natural process of haulm senescence must have important consequences for tuber properties at harvest and may provide a possibility of adjusting the tuber response to impact as also previously described by McGarry et al. (1996). Tubers were pre-harvested from relative immature haulms around 150 days after planting which corresponds to approximately 90 days after emergence. Kolbe and Stephan-Beckmann (1997) stated that at this stage of tuber development until harvest nutrient concentrations $(\mathrm{P}, \mathrm{K}, \mathrm{Ca}, \mathrm{Mg}$ ) and nitrogenous compounds differ only slightly depending on growing conditions, which was also observed in this present study. Despite the fact that nutrients as well as nitrogenous compounds are known to influence blackspot susceptibility the presented results revealed no consistent relationship between nutrient concentration and BSI of tubers. The concentrations of the organic acids namely ascorbic, malic and citric acid are obviously higher in tubers harvested from premature haulms compared to tubers harvested two weeks later as described by Kolbe and StephanBeckmann (1997). This relative higher concentrations might decrease the $\mathrm{pH}$-value and inhibit enzymatic reactions (Pawelzik and Delgado 1999) leading to lesser blackspot development.

Nutrient concentrations in comparison with BSI. During both years of investigation potatoes grew with an adequate supply of all essential nutrients because no nutrient deficiency symptoms were detectable, while tuber quality parameters like starch concentration, dry matter concentration and BSI were significantly different, which might be particularly influenced by some nutrients. Only for K we observed a significant increase in the concentration with increasing specific gravity of pre-harvested tubers in both years of investigation. During these periods of tuber growth the function of $\mathrm{K}$ referred to starch synthesis and dry matter accumulation for normal tuber development and yield response (Westermann et al. 1994b). Within both tested periods of vegetation and storage there was no a single nutrient whose concentration was continuously related to specific gravity and/or BSI. Presented findings are comparable to results from Oswald et al. (1958), Dwelle et al. (1977) and Corsini et al. (1999), who reported no effect of K on BSI presuming an adequate $\mathrm{K}$ supply. This might particularly depend on type of fertilizer because $\mathrm{KCl}$ was able to reduce BSI primary through the component $\mathrm{Cl}$, which reduced the dry matter of tubers grown in dry sandy soils (van Loon and van den Berg 2003/2004), while $\mathrm{K}_{2} \mathrm{SO}_{4}$ (Maier et al. 1986) and $\mathrm{KNO}_{3}$ (Kumar et al. 2007) had no effect on BSI. Also P, Mg, Ca, Fe, B and Se concentrations did not influence BSI permanently. In the case of $\mathrm{P}, \mathrm{Mg}, \mathrm{Ca}, \mathrm{Fe}$ and $\mathrm{B}$ presented findings agreed with studies from Scudder et al. (1950). Adequate fertilization and irrigation ensured sufficient amounts of nutrients around roots and tubers and nutrient 
uptake directly through tuber periderm and into internal tuber tissue (Ca) (Habib and Donelly 2002; Sowokinos 2007) or by mass flow (Ca, Mg, K, Se) and diffusion (K, P, Fe, B) (Bergmann 1993; Brown et al. 2002; Westermann 2005) resulting in required nutrient concentrations for tubers, with the exception of $\mathrm{Ca}$. The $\mathrm{Ca}$ concentration was three times higher in the tubers compared to sufficient level. However, Ca toxicity is relatively unknown (Bergmann 1993) and Ca might be stored as inactive compound in the vacuoles of tubers, while its mobilisation depends on its requirement for physiological processes in tubers (Wulkow et al. 2007). During the whole period of investigation tubers might suffer from an unbalanced $\mathrm{K} / \mathrm{B}$ ratio because of a relative low $\mathrm{B}$ concentration compared to relative high concentration of K. Despite the fact that B deficiency increase blackspot susceptibility (Mondi and Munshi 1993) other deficiency symptoms (rough periderm, necrotic tissue) of tubers were not detectable and the concentration of B was sufficient when comparing with other references.

Moreover, following nutrient interactions of $\mathrm{K} / \mathrm{B}, \mathrm{P} / \mathrm{B}, \mathrm{Ca} / \mathrm{B}$ and $\mathrm{P} / \mathrm{Fe}$ particularly affected BSI of tubers grown on sandy soil. In this process nutrient interactions might occurred in various forms like nutrient displacement in the metabolism during enzymatic reactions which influenced deficiency or surplus symptoms and consequently influencing the quality of potato tubers.

\section{Conclusion}

After typical warm vegetation periods in both years of investigation the time of harvesting and the specific gravity of tubers showed a strong relationship to BSI. However, there were no relation between the absolute concentrations of $\mathrm{K}, \mathrm{P}, \mathrm{Mg}, \mathrm{Ca}, \mathrm{Fe}, \mathrm{B}$ and $\mathrm{Se}$ in potato tubers and their BSI. Nutrient concentrations ranged next to their optimum, with the exception of $\mathrm{Ca}$. That might be particularly based on the fact that proper irrigation enhanced nutrient sufficiency again indicating that the additional application of water accompanied with a use of appropriate fertilization minimize nutrient deficiency on sandy soils. The use of fertilizer should be practiced in adequate rates to get maximum yield. There is not evidence that excessive fertilization will reduce BSI significantly. Moreover, harvesting tubers from haulms that were relatively immature at time of haulm kill might be important for crop management considering minimizing BSI. Nevertheless, this leads to a higher sugar accumulation during storage and decreasing processing quality of tubers if the higher sucrose pool is not managed properly during the post haulm kill and early storage period as mentioned by Corsini et al. (1999).

\section{References}

Aysola P, Anderson P, Langford C H (1987) Wet ashing in biological samples in a micro-

wave oven under pressure using poly (tetrafluoroethylene) vessels. Anal Chem 59:1582-1583 
Bamberg J B, Palta J P, Peterson L A, Martin M, Krueger A R (1998) Fine screening potato (Solanum) species germplasm for tuber calcium. Am J Potato Res 75:181-186

Bartz J A, Locascio S J, Weingartner D P (1992) Calcium and potassium fertilization of potatoes grown in North Florida. II: Effect on Bacterial Soft Rot potential in the tuber, American Potato Journal 69:39-50

Bergmann W (1993) Ernährungsstörungen bei Kulturpflanzen. Verlag Gustav Fischer Jena

Blokhina O, Virolainen E, v Fagerstedt K (2003) Antioxidants, oxidative damage and oxygen deprivation stress: a review. Ann Bot 91:179-194

Brown P H, Bellaloui N, Sah R N, Bassil E, Hu H (2002) Uptake and transport of boron. In: Goldbach H E, Rerkasem B, Wimmer M A, Brown P H, Thellier M, Bell R W (ed) Boron in plant and animal nutrition. Kluwer Academic/Plenum Publishers, New York, pp 103-119.

Bucher M, Kossmann J (2007) Molecular physiology of the mineral nutrition of the potato. In: Vreugdenhill D (ed) Potato biology and biotechnology. Advances and perspectives. Elsevier, Amsterdam, pp 441-470

Corsini D, Stark J, Thornton M (1999) Factors contributing to the blackspot bruise potential of Idaho Potato fields. Am J Potato Res 76:221-226

Davies H V (1998) Physiological mechanisms associated with the development of internal necrotic disorders of potato. Am J Potato Res 75:37-44

Dean B B, Jackowiack N, Nagle M, Pavek J, Corsini D (1993) Blackspot pigment development of resistant and susceptible Solanum tuberosum L. genotypes at harvest and during storage measured by three methods of evaluation. American Potato Journal 70:201-217

Dejmek P, Miyawaki O (2002) Relationship between the electrical and rheological properties of potato tuber tissue after various forms of processing. Biosci Biotechnol Biochem 66:1218-1223

Dilworth L L, Omoruyi F O, Asemota H N (2007) In vitro availability of some essential minerals in commonly eaten processed and unprocessed Caribbean tuber crops. BioMetals 20:37-42

Dwelle R B, Stallknecht G, McDole R E, Pavek J J (1977) Effects of soil potash treatment and storage temperature on blackspot bruise development in tubers of four Solanum tuberosum cultivars. American Potato Journal 54:137-146

Edgell T, Brierley E R, Cobb A H (1998) An ultrastructural study of bruising in stored potato (Solanum tuberosum L.) tubers. Ann Appl Biol 132:143-150 
Griffiths D W, Bain H (1997) Photo-induced changes in the concentrations of individual chlorogenic acid isomers in potato (Solanum tuberosum) tubers and their complexation with ferric ions. Potato Research 40:307-315

Haase N U (2003/2004) Estimation of dry matter and starch concentration in potatoes by determination of under-water weight and near infrared spectroscopy. Potato Research 46:117-127.

Habib A, Donnelly D J (2002) Calcium translocation and accumulation into potato tubers. Potato Res 45:17-24

Heinecke A (2007) Beitrag zur Ermittlung der biochemischen Ursachen der Schwarzfleckigkeit bei Kartoffeln. Ph. D. thesis Georg-August-University Goettingen, Germany. Available via http://webdoc.sub.gwdg.de/diss/2007/heinecke/ accessed 31 Jan 2009

Kirk W W, da Rocha A B, Hollosy S I, Hammerschmidt R, Wharton P S (2006) Effect of soil salinity on internal browning of potato tuber tissue in two soil types. Am J Potato Res 83:223-232

Kolbe H, Stephan-Beckmann S (1997) Development, growth and chemical composition of the potato crop (Solanum tuberosum L.). I. Leaf and stem. Potato Research 40:111-129

Kuennen R W, Wolnik K A, Fricke F L (1982) Pressure Dissolution and real sample matrix calibration for multi element analysis of raw agricultural crops by inductively coupled plasma atomic emission spectrometry. Analytical Chemistry 54:2146-2150

Kumar P, Pandey S K, Singh B P, Singh S V, Dinesh K (2007) Influence of source and time of potassium application on potato growth, yield, economics and crisp quality. Potato Research 50:1-13

Lærke P E (2001) Blackspot bruise in potato tubers. Ph. D. thesis, The Royal Veterinary and Agricultural University, Denmark, ISBN 87-88976-50-5

Lærke P E, Christiansen J, Veierskov B (2002) Colour of blackspot bruises in potato tubers during growth and storage compared to their discolouration potential. Postharvest Biology and Technology 26:99-111

Lampe D (2007) Kali-Düngung im Kartoffelbau- nach unten fehlgesteuert. Kartoffelbau 58:324-327

Leo L, Leone A, Longo C, Lombardi D A, Raimo F, Zacheo G (2008) Antioxidant compounds and antioxidant activity in "Early Potatoes". J Agric Food Chem 56:4154-4163

Locascio S J, Bartz J A, Weingartner D P (1992) Calcium and potassium fertilization of potatoes grown in North Florida. I. Effects on potato yield and tissue Ca and K concentrations, American Potato Journal 69:95-104 
Maier M A, Dahlenburg A P, Fresham A B (1986) Potassium nutrition of irrigated potatoes in South Australia. 3. Effect on specific gravity, size and internal bruising of tubers. Austr J Exp Agr 26:737-744

Makishima A, Nakamura E, Nakano T (1997) Determination of boron in silicate samples by direct aspiration of sample HF solutions into ICPMS. Anal Chem 69:3754-3759

McGarry A, Hole C C, Drew R L K, Parsons N (1996) Internal damage in potato tubers: A critical review. Postharvest Biology and Technology 8:239-258

McNabnay M, Dean B B, Bajema R W, Hyde G M (1999) The effect of potassium deficiency on chemical, biochemical and physical factors commonly associated with blackspot development in potato tubers. Am J Potato Res 75:53-60

McNeal J M, Balistrieri L S (1989) Geochemistry and Occurrence of selenium: An overview. In: Jacobs L W (ed) Selenium in Agriculture and the environment. SSSA pp 1-13

Mondy N I, Munshi C B (1993) Effect of Boron on enzymatic discoloration and phenolics and ascorbic acid contents of potatoes. J Agric Food Chem 41:554-556

Müller A, Wiedenbeck M, van den Kerkhof A, Kronz A, Simon K (2003) Trace elements in quartz- a combined electron microprobe, secondary ion mass spectrometry, laserablation ICP-MS, and cathodoluminescenece study. Eur J Mineral 15:747-763

Muneta P, Kaisaki F (1983) Ascorbic acid-ferrous iron $\left(\mathrm{Fe}^{++}\right)$complexes and after cooking darkening of potatoes. American Potato Journal 62:531-536

Munshi C B, Combs G F, Mondy N I (1990) Effect of selenium treatment on the nitrogenous constituents of the potato. J Agric Food Chem 38:2000-2002

Nitsch A (2005) Aufnahmeverlauf von Makro- und Mikronährstoffen in Kartoffelknollen. Conference of the Society of Plant Breeding e.V. (GPZ) from $16^{\text {th }}$ to $17^{\text {th }}$ November 2005 Goettingen, Germany

Nzaramba M N, Bamberg J B, Miller J C Jr (2007) Effect of propagule type and growing environment on antioxidant activity ant total phenolics content in potato germplasm. Am J Potato Res 84:323-330

Oswald J W, Lorenz O A, Bowman T, Snyder M, Hall H (1958) Potato fertilization and internal blackspot in Santa Maria Valley. Fertilization studies show potash deficiency to be closely linked with the incidence of internal disorder of potatoes. Calif Agric 12:8-10

Pawelzik E, Delgado E (1999) Wirkung von Trockenstress auf die Verfärbungsneigung von Kartoffelknollen. Kartoffelbau 9/10:358-360

Pellegrini N, Serafini M, Colombi B, del Rio D, Salvatore S, Bianchi M, Brighenti F (2003) Total antioxidant capacity of plant foods, beverages and oils consumed in Italy assessed by three different in vitro assays. J Nutr 133:2812-2819 
Peterson R L, Barker W G, Howarth M J (1985) Development and structure of tubers. In: Li P H (ed) Potato Physiology, Academic Press, Orlando, Fl, pp124-152

Putz B (1998) Kartoffeln: Züchtung, Anbau, Verwertung. Verlag Behr's, Hamburg

Rogozińska I, Wszelaczyńska E, Wichrowska D (2005) Effect of bioelements (Mg, N, K) and herbicides on vitamin $\mathrm{C}$ content in potato tubers. Part I: Vitamin C content in potato tubers immediately after harvest. Elementology 10:999-1008

Scanlon M G, Pang C H, Biliaderis C G (1996) The effect of osmotic adjustment on the mechanical properties of potato parenchyma. Food Res Int 29:481-488

Scudder W T, Jacob W C, Thompson H C (1950) Varietal susceptibility and the effect of potash on the incidence of blackspot in potatoes. Proc. Am. Soc.Hortic. Sci. 56:343-348

Seppänen M, Turakainen M, Hartikainen H (2003) Selenium effects on oxidative stress in potato. Plant Sci 165:311-319

Simmons K E, Kelling K A (1987) Potato responses to calcium application on several soil types. American Potato Journal 64:119-136

Sowokinos J R (2007) Internal physiological disorders and nutritional and compositional factors that affect market quality. Advances and perspectives, In: Vreugdenhill D (ed) Potato biology and biotechnology. Advances and perspectives. Elsevier, Amsterdam, pp $501-523$

Storey M (2007) The harvested crop. In: Vreugdenhill D (ed) Potato biology and biotechnology. Advances and perspectives. Elsevier, Amsterdam, pp 441-470

Sulaiman M I (2005) Effect of calcium fertilization on the Quality of potato tubers (Solanum tuberosum L.) cv. Saturna. PhD. Thesis, Georg-August-University Goettingen, Germany ISBN 3-86537-598-7

Terry N, Zayed A M, Souza M P, Tarun A S (2000) Selenium in higher plants. Annual Review of Plant Physiology and Pant Molecular Biology. 51:401-432

Thornton R E, Timm H (1990) Influence of fertilizer and irrigation management on tuber bruising. American Potato Journal 67:45-54

Turakainen M, Hartikainen H, Ekholm P, Seppänen M (2006) Distribution of selenium in different biochemical fractions and raw darkening degree of potato (Solanum tuberosum L.) tubers supplemented with selenate. J Agric Food Chem 54:8617-8622

Tzeng K C, Kelmann A, Simmons, Kelling K A (1986) Relationship of calcium nutrition to internal Brown Spot of potato tubers and sub-apical necrosis of sprouts. American Potato Journal 63:87-97

van Loon C D, van den Berg W (2003/2004) The effect of chloride fertilization on blackspot susceptibility and other quality characteristics and on yield of potato. Potato Research 46:147-154 
Westermann D T (2005) Nutritional requirements of potatoes. Am J Potato Res 82:301-307

Westermann D T, James D W, Tindall T A, Hurst R L (1994b) Nitrogen and potassium fertilization of potatoes: sugars and starch. Am Potato J 71:433-453

Westermann D T, Tindall T A, James D W, Hurst R L (1994a) Nitrogen and potassium fertilization of potatoes: yield and specific gravity. Am Potato J 71:417-431

White P J, Wheatley R E, Hammond J P, Zhang K (2007) Minerals, soils and roots. In: Vreugdenhill D (ed) Potato biology and biotechnology. Advances and perspectives. Elsevier, Amsterdam, pp 739-752

Wilhelm E, Tegge G, Vitte V (1983) vergleichende Untersuchungen über Standardmethoden zur Phosphorbestimmung. Starch- Stärke 8:282-287

Workman M, Holm D G (1984) Potato clone variation in Blackspot and soft rot susceptibility, redox potential, ascorbic acid, dry matter and potassium. American Potato Journal 61:723-733

Wulkow A, Pawelzik E, Peters R, Haase N U (2009) Blackspot bruise formation in potato (Solanum tuberosum) tubers in relation to the components determining tuber specific gravity (in preparation)

Wulkow A, Sulaiman M I, Pawelzik E (2007) Calciumdüngung zu Kartoffeln. Einfluss der Calciumfraktionen in der Kartoffelknolle. Kartoffelbau 58:78-83

Yahraus T, Chandra S, Legendre L, Low P S (1995) Evidence for mechanically induced oxidative burst. Plant Physiol 109:1259-1266 


\title{
5. Blackspot bruise formation in potato (Solanum tuberosum) tubers in relation to the components determining tuber specific gravity
}

\begin{abstract}
Table potato (Solanum tuberosum) tubers with a range of specific gravities from $<1.055$ to $>1.095 \mathrm{~kg} \mathrm{~L}^{-1}$ from a three years field experiment were investigated. Genetic and environmental factors had little influence on the development of tubers with different specific gravities. To a greater extend the accumulation of dry matter and water during different phases of tuberization determined the specific gravities of mature tubers of one cultivar or plant. Mechanical impacts on harvested and stored potato tubers initiated blackspot bruise formation. Inconsistent results occurred in many reports if intracellular membrane rupture is necessary to form blackspot bruise or not. The presented results showed that blackspot bruise formation might occur without membrane rupture because cell necrosis or cell deaths were not detected. Thus presented findings compared to literature outcomes showed that mechanical impact on potato tubers led to open mechanosensitive channels of intracellular membranes, and therefore to blackspot bruise formation without intracellular membrane rupture.
\end{abstract}

Keywords: dry matter, starch content, pectin

\section{Introduction}

Table potatoes are consumed worldwide, while their acceptance as food differs due to regional habits. Additionally, convenience food becomes more accepted rather than nonprocessed tubers (Haase 2008). This trend is noticed all over the world, especially in Europe and North America where 20 to $50 \%$ of the daily potato consumption consist of processed tubers. The quality of these products depends on the available raw potato tubers and therefore on growing conditions as well as harvesting, handling and storage operations (Keijbets 2008). These procedures enhance the possibility to damage potato tubers mechanically resulting in blackspot bruise, which is known to reduce tuber quality and therefore, its utilization. Since many years the reduction of blackspot bruise susceptibility of potato tubers is a topic of interest of scientific research. Tuber impact susceptibility include bruise threshold and bruise resistance. Bruise threshold is the impact at which blackspot occurs and bruise resistance is defined as the ratio of bruising energy to the resulting bruise volume (van Eck 2007). Blackspot describes a dark discolouration of the cortical parenchyma. After impact phenols, primarily monophenols like tyrosine and/or diphenols like chlorogenic acid undergo a series of oxidative reactions initiated by the copper containing enzyme polyphenoloxidase (PPO) to form melanin. The pigment is responsible for the dark discolouration found in bruised tissue. In non-bruised tissue PPO and its substrates are separated by intracellular compartmentation. PPO is largely associated with the plastids and the substrates are located in the vacuole. Impact on tissue causes a coincidence of PPO and its substrates, leading to blackspot bruise formation in the presence of oxygen (Edgell 
et al. 1998). This bruise threshold depends on the stage of crop growth and the biochemical potential of the tuber to produce superoxide radicals (Story 2007). Bruise resistance refers to the mechanical properties of the tissue, which are influenced by tuber specific gravity (Baritelle and Hyde 2003; Scanlon et al. 1996). Specific gravity is generally assumed to be a cultivar characteristic (Laboski and Kelling 2007) and reflects the dry matter concentration of potato tubers (Haase 2003/2004). The term dry matter refers to all substances of the potato tuber, except water (Putz 1998) and includes approximately $80 \%$ starch, $10 \%$ cytoplasmatic components and $10 \%$ cell wall material (Lærke 2001). Many studies mentioned the starch concentration as the predominant factor, which influences the blackspot susceptibility (e.g. Baritelle and Hyde 2003; Lærke 2001; Scanlon et al. 1996). However, information are still rare if this relation also exists among different cultivars, environmental factors and storage conditions. Also the cell wall cohesion and the firmness of the cell wall might affect any impact on the mechanical properties of the tissue and internal structural changes (Alvarez et al. 2000; Story 2007). The cell wall cohesion and the firmness of the cell wall depend on its structure (Taylor et al. 2007) and its pectin concentration (Keijbets and Pilnik 1974). Pectin is a component of the middle lamella of the primary cell wall (Braun 1989) belonging to the group of polygalacturonic acids and varying in its structure of side chains. The side chains consist of arabinose and galactose and a complex ramified network of mono saccharides (Kohorn 2000). Depending on the complexity and the degree of esterification of the side chains the physiological properties of pectin are modified (Guillotin 2005). Non-esterified carboxyl groups of adjacent side chains are linked by multivalent ions like $\mathrm{Ca}^{2+}$ and $\mathrm{Mg}^{2+}$, which might predefine cell wall firmness (Braun 1989). The cell wall structure (Taylor et al. 2007) and its pectin concentration (Braun 1989; Moledina et al. 1981; van Eck 2007) determine the texture and quality of processed tubers. However, from our knowledge, no results are available if the pectin concentration of potato tubers influences their blackspot susceptibility.

The aim of this study was to investigate the components which are attributed to tubers specific gravity and their influence on blackspot bruise susceptibility of table potato cultivars, respectively.

\section{Material and methods}

Material. Eight cultivars of table potatoes as Adretta, Afra, Gala, Granola, Lolita, Marabel, Nicola and Renate were grown near Dethlingen, Germany with conventional farming methods during three vegetation periods in 2005, 2006 and 2007. From harvested plants tubers with a similar size of 40 to $50 \mathrm{~mm}$ were selected to get comparable results. Analyses were performed in tubers after harvest and in tubers stored for five and eight months, respectively, at $4^{\circ} \mathrm{C}$ and $95 \%$ relative humidity. Moreover, the tubers were divided into six groups of specific gravity ranking from $<1.055$ to $>1.095 \mathrm{~kg} \mathrm{~L}^{-1}$, with 0.01 increments. The separation was performed directly before testing (Wright et al. 2005). For this purpose tubers were plunged in different vessels with demineralised water and adequate $\mathrm{NaCl}$ - con- 
centrations, respectively. Furthermore, the samples were divided into two parts. Tubers from the first part were used to determine their blackspot index (BSI). Tubers from the second part were used for analysis. Samples were sliced and used fresh or were shockfrozen in liquid nitrogen and stored at $-30{ }^{\circ} \mathrm{C}$ until testing or freeze-drying (Epsilon 2-40, Christ, Germany).

Analyses. Blackspot index (BSI) of whole potato tubers of different specific gravities was determined according to the method of the German "Bundessortenamt" (BSA) (Federal Plant Variety Office). The tubers were cooled down to 4 to $5^{\circ} \mathrm{C}$ and mechanical treated for $50 \mathrm{~s}$ in a vegetable washing machine with a rotating drum (Flottwerk H.J. Dames GmbH \& Co. KG, Rotenburg a. d. F., Germany) with three replications. Each replication consisted of an amount of tubers $\left(\Sigma_{\text {tuber }}\right)$ adequate to a volume of $6 \mathrm{~L}$. Afterwards, treated tubers were stored at room temperature for 48 hours. For BSI determination they were cut into halves length-wise and a half tuber was evaluated visually using a scale with four scores of discolouration: No discolouration indicated no blackspot development and therefore no blackspot bruise susceptibility $\left(\Sigma_{1}\right)$. Discolouration till quarter of length and less than 5 $\mathrm{mm}$ in-depth showed slight blackspot bruise susceptibility $\left(\Sigma_{2}\right)$. Medium blackspot bruise susceptibility corresponded to a discolouration till quarter of length and above $5 \mathrm{~mm}$ indepth or a half of the length and maximal $5 \mathrm{~mm}$ in-depth $\left(\Sigma_{3}\right)$. Serious blackspot bruise susceptibility was indicated by a stronger discolouration $\left(\Sigma_{4}\right)$. The blackspot bruise susceptibility, expressed as the percentage of discoloured tubers was calculated from following equation (1):

$$
\operatorname{BSI}(\%)=\left(0.3 \sum_{2}+0.5 \sum_{3}+\sum_{4}\right) / \sum_{\text {tuber }} * 100
$$

BSI: blackspot index (\%)

$\Sigma: \quad$ amount of analysed tubers

The dry matter concentration of potato tubers was analysed according to Naumann and Bassler (1976). Starch analyses referred to the ICC- Standard method no. 123/ 1 (2006).

The pectin concentrations of potato tubers were analysed in potato tuber periderm, nonperiderm as well as in whole tubers differing in their specific gravity. Samples were prepared from fresh shock-frozen tissues. First of all the extraction of the ethanol insoluble cell wall material has taken place with $60 \mathrm{~g}$ sliced tissue $\left(\right.$ tissue $_{\text {fresh }}$ ), which was mixed with denatured ethanol using an ultra-turrax (Janke \& Kunkel, IKA lab technics, Germany) (Keijbets and Pilnik 1974). Two grams of the degreased ethanol insoluble cell wall material (tissue $_{\text {degreased }}$ ) were cleaned up form starch using cold demineralised water and a nylon sieve with a porosity of $>100 \mu \mathrm{m}$ (tissue starch-free$_{\text {) }}$ ) and dried in a compartment dryer (MMM-

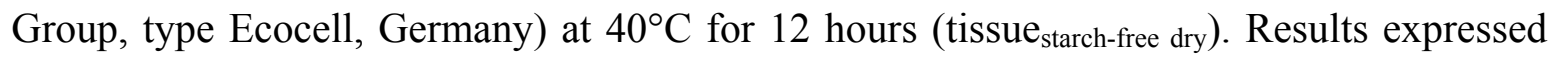
the cell wall material solids lager than $100 \mu \mathrm{m}$ calculated from following equation (2): 
dry cell wall material $\left(\mathrm{g} \mathrm{kg}^{-1} \mathrm{FM}\right)=\left(\frac{\text { tissue }_{\text {deg reased }} * \text { tissue }_{\text {starch-freedry }} * 100}{\text { tissue }_{\text {starch-free }} * \text { tissue }_{\text {fresh }}}\right) * 1000$

The pectin concentration of the cell wall material and its degree or esterification were analysed using the $\mathrm{Cu}^{2+}$ ion exchange method. Thereby filtrates of total pectin (filtrate A) and filtrates of the esterified pectin (filtrate B) were performed as described in detail in some evidences (Pardede 2004; Braun 1989; Längerer et al. 1978; Bäuerle et al. 1977; Tibensky et al. 1963) from $0.1 \mathrm{~g}$ dry cell wall material. Later we used a solution of $0.5 \%$ $\mathrm{C}_{14} \mathrm{H}_{22} \mathrm{~N}_{4} \mathrm{O}_{2}$ to change the $\mathrm{Cu}^{2+}$ ions of the pectin filtrates against $\mathrm{H}^{+}$ions. Depending on the amount of $\mathrm{Cu}^{2+}$ the $\mathrm{C}_{14} \mathrm{H}_{22} \mathrm{~N}_{4} \mathrm{O}_{2}$ solution became blue coloured, whereby its absorbance was measured at $560 \mathrm{~nm}$ with an UV-Vis spectral system (HP 8453, Germany). $\mathrm{CuSO}_{4} * 5 \mathrm{H}_{2} \mathrm{O}$ standard solutions with a $\mathrm{Cu}^{2+}$ concentration from 0.5 to $5 \mathrm{mg} \mathrm{L}^{-1}$ were used to quantify the $\mathrm{Cu}^{2+}$ concentration of the filtrates. Results reflecting the concentration and the degree of esterification of pectin in potato tuber tissue were calculated with following equations (3) and (4):

$$
\begin{aligned}
& \text { pectin }\left(g \mathrm{~kg}^{-1} \mathrm{FM}\right)=\left[\left(\frac{190 \mathrm{~B}-14 \mathrm{~A}}{317.5 * 1000 * W}\right) * 1000 * 5\right] *\left(\frac{\text { dry cell wall material }\left(g \mathrm{~kg}^{-1} \mathrm{FM}\right)}{1000}\right) \\
& \text { esterifica tion }(\%)=[(B-A) * 100] / B
\end{aligned}
$$

$\mathrm{A}=$ total pectin concentration of filtrate $\mathrm{A}$

$B=$ esterified pectin of filtrate $B$

$\mathrm{W}=$ amount of the analysed dry cell wall material

The non-pectin components presented in the fresh matter of potato tuber tissue were calculated as the difference between the dry cell wall material (2) and the pectin concentration (3).

The concentration of other (cytoplasmatic) components in the fresh matter of potato tuber tissue is figured as the differences between the dry matter concentration and the summarized concentration of the starch and the dry cell wall material.

Statistics. The obtained data were analysed with Sigma Stat statistical program (STAT32). All data sets were tested for normal distribution and variance homogeneity $(p=0.05)$. Following analyses of variance were calculated for cultivar and specific gravity. Calculated means were compared by Tukey-test $(\mathrm{p} \leq 0.05)$. Correlation and multiple regression procedures between normally distributed quality parameters were performed using Pearson correlation coefficients.

\section{Results}

Distribution of tubers with different specific gravities within each cultivar. During the vegetation period of potato crops the specific gravity of tubers might differ due to maturing 
time of cultivars, physiological tuber age and environmental and pathogenic impact. Also tuber skin structures, gas concentration in the periderm and storage conditions might affect tuber specific gravity (Laboski and Kelling 2007). With respect to these facts potato tubers of the studied cultivars were separated according to their specific gravities after harvest and storage periods (Fig. 5.1 [A, I-III]; Fig. 5.2 [A, IV-VI]; Fig. 5.3 [A, VII-IX]; annex, Tab. 10.43 - 10.45).

Cultivars harvested 2005 generated tubers with specific gravities from $<1.055$ to $>1.095 \mathrm{~kg}$ $\mathrm{L}^{-1}$ (Fig. 5.1). The amount of tubers differing in their specific gravities was normal distributed within each cultivar, excepted tubers of cv. Marabel. After harvest (Fig. 5.1 [A, I]) tubers with a specific gravity of $1.056-1.065 \mathrm{~kg} \mathrm{~L}^{-1}$ were performed in cvs. of Gala and Marabel mostly. Tubers with a specific gravity of $1.066-1.075 \mathrm{~kg} \mathrm{~L}^{-1}$ dominated in all cvs., excepted cv. Lolita, because in cv. Lolita and also in cvs. Afra and Adretta tubers with a specific gravity of $1.076-1.085 \mathrm{~kg} \mathrm{~L}^{-1}$ were generated. Also tubers with a specific gravity of 1.086-1.095 kg L $\mathrm{kg}^{-1}$ were produced in the cv. Afra. After storage periods of five (Fig. 5.1 [A, II]) and eight months (Fig. 5.1 [A, III]) we observed no significant changes in the distribution of tubers differing in their specific gravity within each cultivar. In all cultivars tubers with a specific gravity of $1.066-1.075 \mathrm{~kg} \mathrm{~L}^{-1}$ were prevalent.

Cultivars harvested 2006 again originated tubers with specific gravities from $<1.055$ to $>1.095 \mathrm{~kg} \mathrm{~L}^{-1}$ (Fig. 5.2). The amount of tubers differing in their specific gravities was normal distributed within each cultivar. Just yet a high amount of tubers with a specific gravity $<1.055 \mathrm{~kg} \mathrm{~L}^{-1}$ significantly occurred in the cv. Gala (Fig. 5.2 [A, IV]). Contrary to 2005 all cultivars generated many tubers with a specific gravity of $1.056-1.065 \mathrm{~kg} \mathrm{~L}^{-1}$, excepted cv. Afra. However, in all cultivars tubers with a specific gravity of $1.066-1.075 \mathrm{~kg} \mathrm{~L}^{-1}$ dominated. A significant high amount of tubers with a specific gravity of $1.076-1.085 \mathrm{~kg}$ $\mathrm{L}^{-1}$ was observed in cvs. Adretta, Afra, Marabel and Renate. Also a high amount of tubers with a specific gravity of 1.086-1.095 $\mathrm{kg} \mathrm{L}^{-1}$ was generated in cvs. Adretta and Afra. After a storage period of five months (Fig. 5.2 [A, V]) the amount of tubers with a specific gravity of 1.076-1.085 $\mathrm{kg} \mathrm{L}^{-1}$ increased in cv. Lolita also. The same observation was made for the cv. Granola after eight months (Fig. 5.2 [A, VI]) of storage.

Cultivars harvested 2007 once more originated tubers with specific gravities from $<1.055$ to $>1.095 \mathrm{~kg} \mathrm{~L}^{-1}$ (Fig. 5.3). The amount of tubers differing in their specific gravities was normal distributed within each cultivar. The highest amount of tubers with a specific gravity of 1.056-1.065 $\mathrm{kg} \mathrm{L}^{-1}$ was performed from all cultivars, excepted cvs. Adretta, Afra, and Lolita (Fig. 5.3 [A, VII]). Nevertheless, in all cultivars the amount of tubers with a specific gravity of $1.066-1.075 \mathrm{~kg} \mathrm{~L}^{-1}$ was prevalent. Many tubers with a specific gravity of 1.076-1.085 kg L $\mathrm{kg}^{-1}$ were also performed in cvs. Adretta, Afra, Lolita and Nicola. Previous cvs. generated a significant high amount of tubers with specific gravities of 1.086-1.095 kg $\mathrm{L}^{-1}$ also. After storage periods of five (Fig. 5.3 [A, VIII]) and eight months (Fig. 5.3 
$[\mathrm{A}, \mathrm{IX}])$ in all cultivars the amount of tubers with a specific gravity of $1.076-1.085 \mathrm{~kg} \mathrm{~L}^{-1}$ dominated.

Blackspot index and tuber components attributed to specific gravity. Results of BSI, tuber dry matter concentration and the concentrations of its determined components are shown in Figure 5.1 [B, I-III], Figure 5.2 [B, IV-VI], Figure 5.3 [B, VII-IX] and in the annex (see Tab. 10.1 - 10.3, 10.46 - 10.61). For BSI and tuber dry matter concentration only standard deviations are mentioned, because the concentrations of dry matter attributed components were not significantly different between tubers differing in their specific gravity, except the starch concentration. The obtained amount of tubers with specific gravities $<1.055 \mathrm{~kg} \mathrm{~L}^{-1}$ and $>1.095 \mathrm{~kg} \mathrm{~L}^{-1}$ was sometimes less than a required volume of $6 \mathrm{~L}$, therefore BSI tests were not performed and hence no data shown in the figures. The concentrations of dry matter attributed components were not determined after harvest in 2006 (Fig. 5.2 [B, IV]) and after harvest in 2007 (Fig. 5.2 [B, VII]) because of a lack of sufficient amount of tuber samples.

In general, tubers with different specific gravities had significantly different dry matter concentrations. However, the tuber dry matter concentration was not significantly different between cultivars, vegetation and storage period. For example, tubers with specific gravities $<1.055 \mathrm{~kg} \mathrm{~L}^{-1}$ consisted of 14 to $15 \%$ dry matter, which is significant different from tubers with a specific gravity of 1.056-1.065 $\mathrm{kg} \mathrm{L}^{-1}$ comprised 15 to $17 \%$ dry matter. Significant higher dry matter concentrations of $19 \%$ were determined in tubers with specific gravities of $1.066-1.075 \mathrm{~kg} \mathrm{~L}^{-1}$ and dry matter concentrations of 20 to $21 \%$ in tubers with a specific gravity of 1.076-1.085 $\mathrm{kg} \mathrm{L}^{-1}$, respectively. Also significant higher dry matter contents of 23 to $24 \%$ were analysed in tubers with specific gravities of $1.086-1.095 \mathrm{~kg} \mathrm{~L}^{-1}$, as well as $>24 \%$ in tubers with a specific gravity of $>1.095 \mathrm{~kg} \mathrm{~L}^{-1}$.

The dry matter concentration of tubers was significantly correlated to their $\mathrm{BSI}\left(\mathrm{P}<0.01, \mathrm{R}^{2}\right.$ $=0.36$ ) during the entire period of investigation.

Clearly, starch was the main component of the dry matter $\left(\mathrm{P}<0.01, \mathrm{R}^{2}=0.74\right)$ and increased with increasing specific gravity $\left(\mathrm{P}<0.01, \mathrm{R}^{2}>0.38\right)$. In a specific gravity range the starch concentration was the same irrespective of cultivar, year and storage period, excepted in 2005 after eight months of storage. In particular, less than $10 \%$ starch was concentrated in tubers with specific gravities $<1.055 \mathrm{~kg} \mathrm{~L}^{-1}$, about $11 \%$ starch contained tubers with a specific gravity of $1.056-1.065 \mathrm{~kg} \mathrm{~L}^{-1}, 13 \%$ starch was accumulated in tubers with specific gravities of 1.066-1.075 kg L-1 and $14 \%$ starch was determined in tubers with a specific gravity of 1.076-1.085 $\mathrm{kg} \mathrm{L}^{-1}$. A significant higher starch concentration of $16 \%$ was found in tubers with a specific gravity of $1.086-1.095 \mathrm{~kg} \mathrm{~L}^{-1}$, as well as $18 \%$ in tubers with a specific gravity of $>1.095 \mathrm{~kg} \mathrm{~L}^{-1}$. The starch concentrations of tubers was significantly correlated to their $\mathrm{BSI}\left(\mathrm{P}<0.01, \mathrm{R}^{2}=0.37\right)$ during the term of validity.

The concentration of dry cell wall material was particularly different between tubers of different specific gravities and represented about 0.3 to $16 \%$ of the dry matter concentra- 
tion. However, no relation between the concentration of dry cell wall material and BSI was measurable $(\mathrm{P}>0.05)$. Pectin is one component of dry cell wall material and its concentrations ranged between 0.04 and $0.6 \%$ and remains invisible low in most presented figures. Also its concentration was very low compared to the calculated residual cell wall compounds, referred as non-pectin concentration. Neither the pectin concentration nor the nonpectin concentration reflected tubers blackspot susceptibility $(\mathrm{P}>0.05)$. Depending on the period of investigation the degree of esterification of pectin differed slightly between tubers differing in their blackspot susceptibility. Approximately $20 \%$ to $60 \%$ of the pectin chains were esterified. Nevertheless, the degree of esterification of the pectin was not correlated to the BSI of tubers (annex, Tab. $10.59-10.61$ ).

The other components refer to the cytoplasmatic compounds of potato tuber tissue and were calculated as the differences between the dry matter concentration and the summarized concentration of the starch and the dry cell wall material. The concentration of the cytoplasmatic components ranged between $2 \%$ and $7 \%$ depending on tubers specific gravity. However, a significant increasing concentration of the cytoplasmatic components with increasing specific gravity was not observed and the calculated concentration of the cytoplasmatic components were not related to tubers BSI $(\mathrm{P}>0.05)$. 

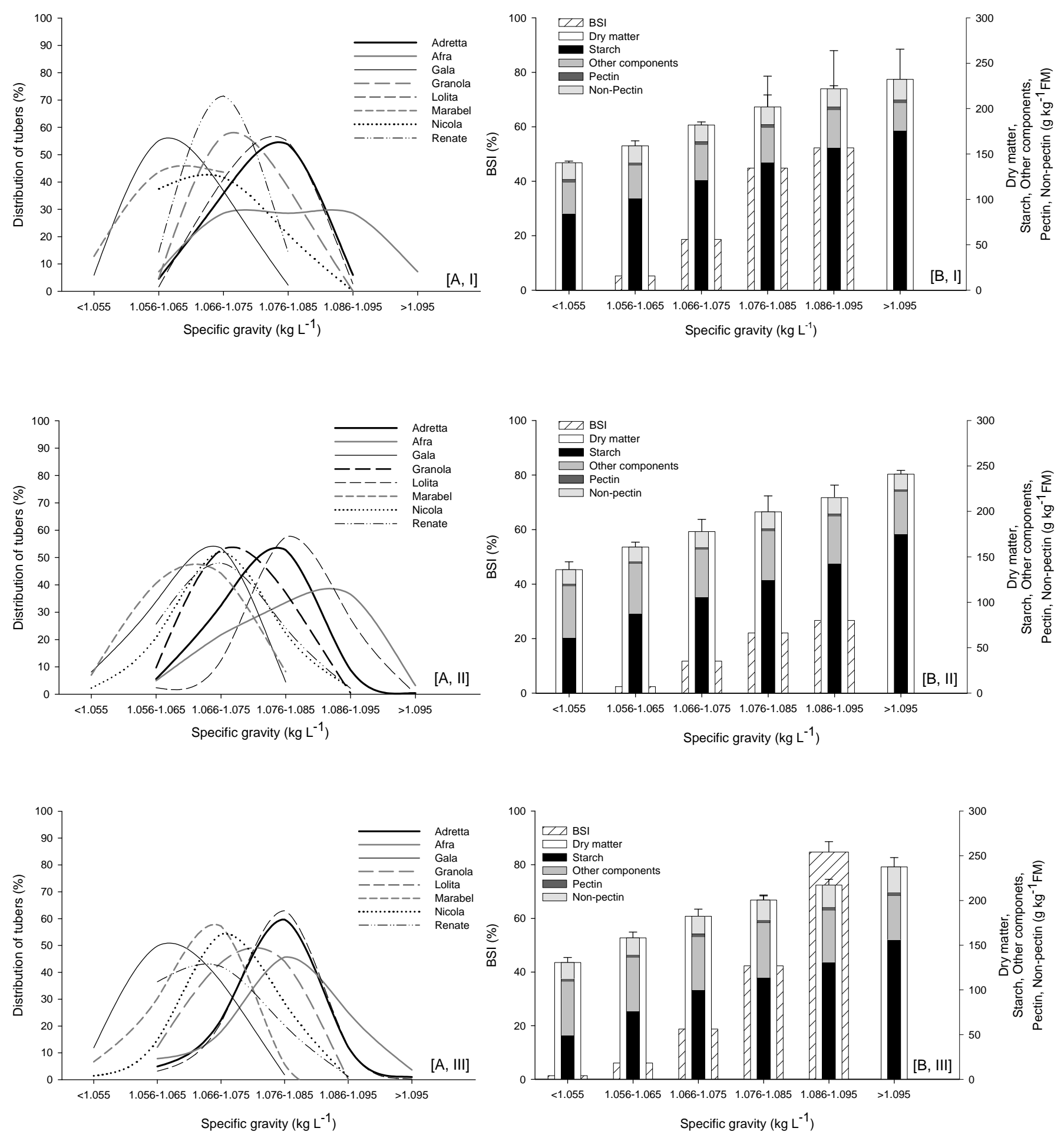

Figure 5.1 Distribution of tubers (\%) differing in their specific gravity within a cultivar (A), blackspot index (BSI, \%) and tuber components attributed to specific gravity (B) of potato tubers harvested 2005 (I) and stored for five (II) and eight (III) months, respectively 

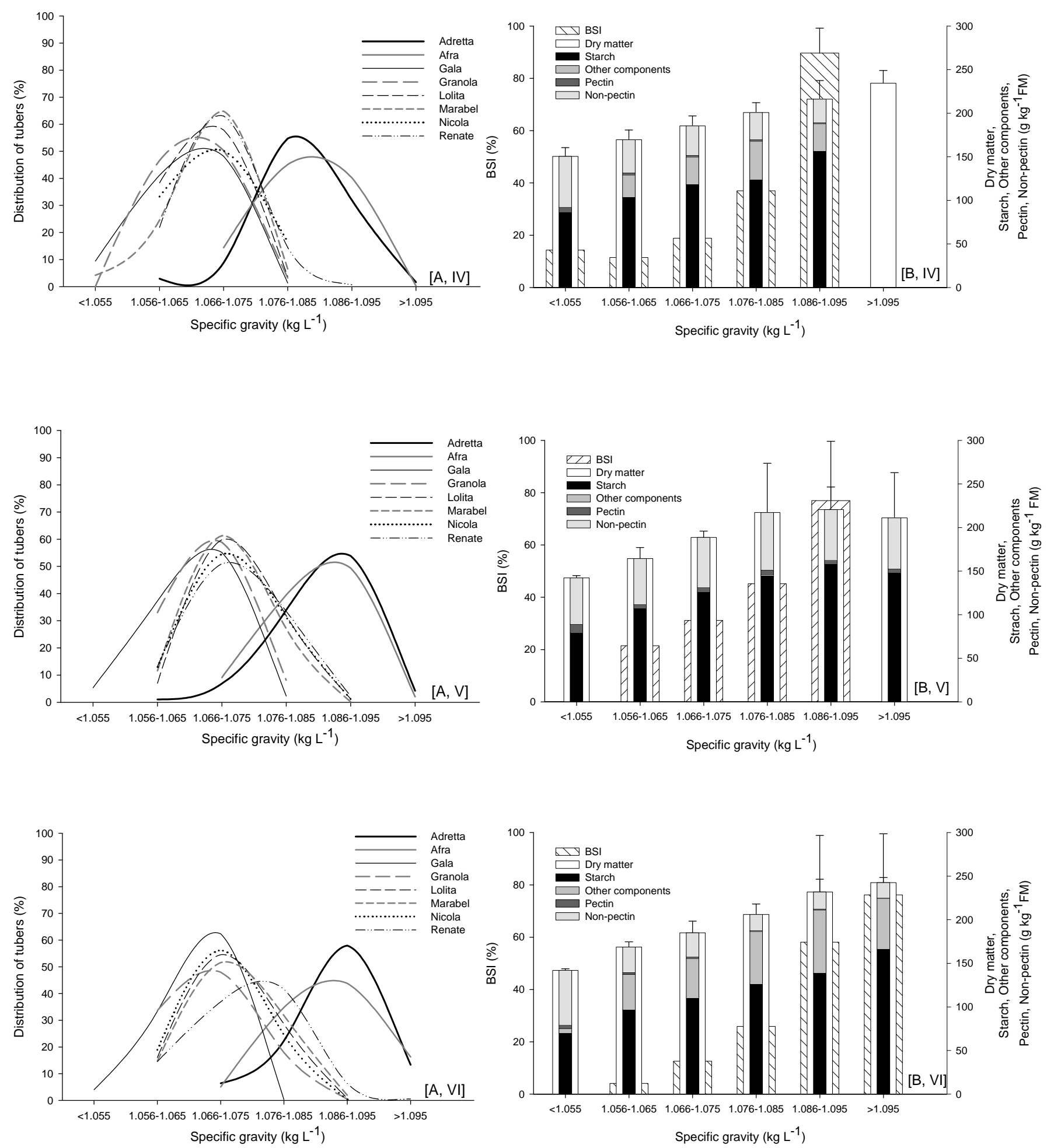

Figure 5.2 Distribution of tubers (\%) differing in their specific gravity within a cultivar (A), blackspot index (BSI, \%) and tuber components attributed to specific gravity (B) of potato tubers harvested 2006 (IV) and stored for five (V) and eight (VI) months, respectively 

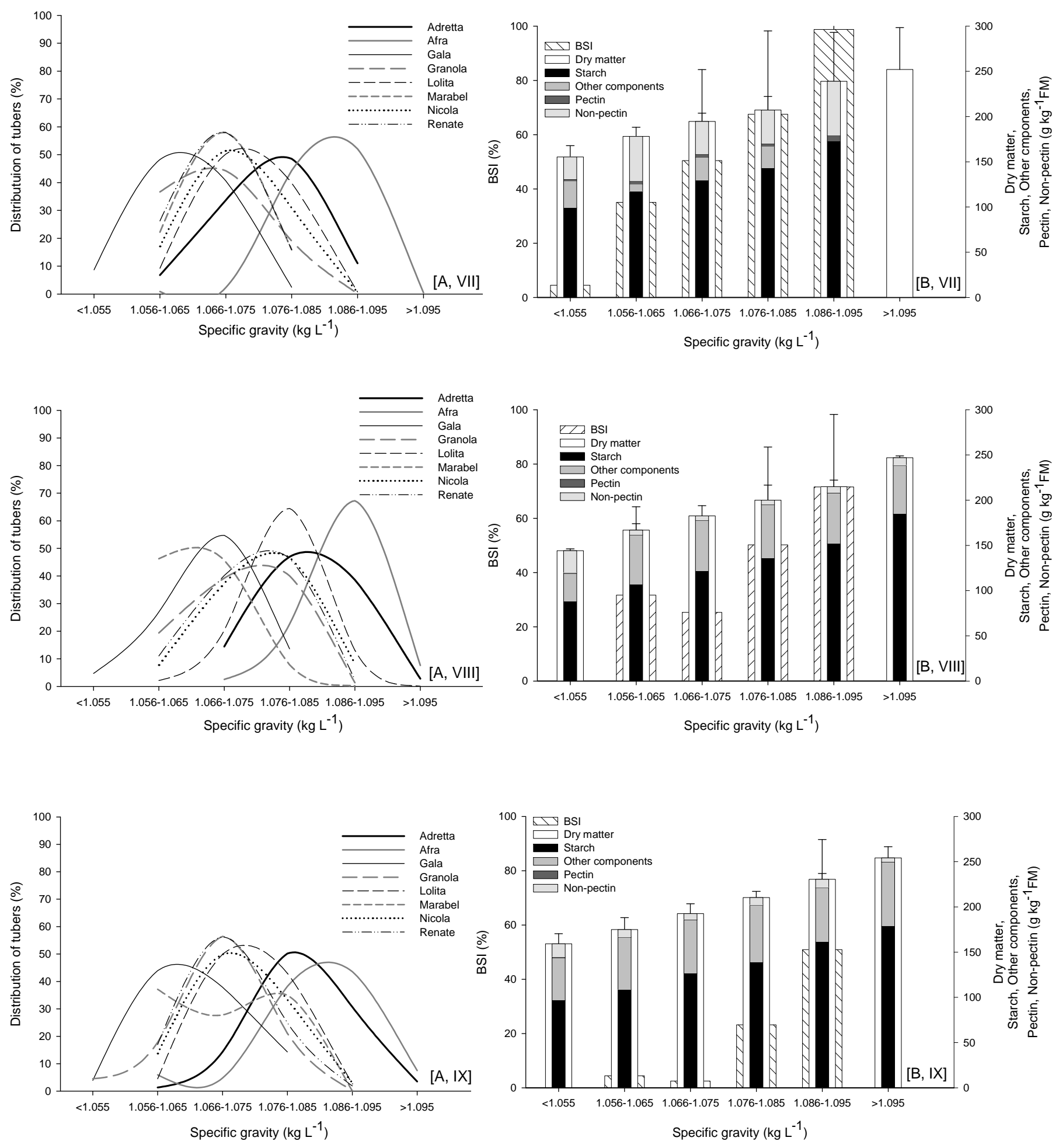

Figure 5.3 Distribution of tubers (\%) differing in their specific gravity within a cultivar (A), blackspot index (BSI, \%) and tuber components attributed to specific gravity (B) of potato tubers harvested 2007 (VII) and stored for five (VIII) and eight (IX) months, respectively 


\section{Discussion}

Distribution of tubers with different specific gravities within each cultivar. In general, the specific gravity of potato tubers is assumed to be special attributed to cultivars (Laboski and Kelling 2007). However, presented investigations indicated that the specific gravity of tubers ranged between $<1.055$ and $>1.095 \mathrm{~kg} \mathrm{~L}^{-1}$ independent of given cultivars. This is due to the fact that growing and storage conditions might overrule some conditions of cultivar dependent specific gravity formations as mentioned by Laboski and Kelling (2007). Tubers harvested from the same field differed in their specific gravity up to 0.008 to 0.010 units only. Investigated cultivars where grown in single hills in the same field and performed an individual set of specific gravities, whereby the sets of specific gravities of cultivars differed form each other about 0.010 units. The observed range of specific gravities about 0.039 units within a single cultivar and hill is quite possible and followed the hierarchy of tubers to accumulate dry matter and water as observed by Bussieres (1993), Kolbe and Stephan-Beckmann (1997) and Struik et al. (1991). This sink-strengh hierarchy is greatly affected by competitory relationships between plants and plant individual distributed stems and are partially open to influence as described by Struik et al. (1990) in detail.

Blackspot index and tuber components attributed to specific gravity. The BSI of tubers of various specific gravities was not correlated with the concentrations of dry cell wall material, pectin or non-pectin components (celluloses, hemicelluloses). Both parameters affect cell wall thickness and cell wall firmness in potato tubers (Bush et al. 2001). Therefore present results indicated that neither cell wall thickness nor cell wall firmness was attributed to tuber blackspot bruise susceptibility. Other types of bruise like crushing, shatter and cracking lead to visible cell wall rupture (Baritelle and Hyde 2003) but blackspot bruise is invisible until the tuber is peeled because the tuber skin is not damaged or broken after impact (Wright et al. 2005). Nevertheless, blackspot bruise formation depends on a linkage between PPO and substrates normally stored in different cell compartments divided by cell membranes. Therefore, the consistence of the cell membranes characterise blackspot susceptibility of the tuber. Many reports assumed a decompartmentation of PPO and substrates, while the cell membranes were ruptured during impact (Story 2007; van Eck 2007; van Loon and van den Berg 2003/2004). In the present investigations structural damages, which lead to necrosis or cell death were not observed. Moreover, tubers were vital and turgid after harvest as well as after five and eight months of storage (Wulkow et al. in preparation) independent of their blackspot bruise susceptibility. Presented findings indicated an intact cell metabolism of potato tubers after impact and blackspot bruise formation. According to the presented results some authors studied the appearance of cell membrane using different microscope techniques and they detected no cell membrane rupture after impact (Barkhausen 1978; Edgell et al. 1998; Reeve 1968). We assume that the impact leading to blackspot bruise is a kind of mechanical stress, which might be focused on mechanosensitive channels as described by Larcher (1994) and Yahraus (1995) in de- 
tail. Mechanosensitive channels physically connect the extracellular matrix with the cytoskeleton (Wang et al. 1993). Signal transformation and signal transduction occurs only when the cytoskeleton is connected to the cell surface (Chen and Ingber 1999). These conditions are present in turgid cells. Pressure increase within a given unit raise channel opening of about $10 \%$, whereas maximal possible elastic changes of cell membranes increase the channel opening only about with $2 \%$ (Garrill et al. 1996). Therefore, the cultivar specific arrangement of the cytoskeletal components and local distortions in the lipid bilayer might influence channel opening. The number and the arrangement of the mechanosensitive channels in the membrane may determine quantitative response of the cell to qualitative signals (Garrill et al. 1996). The activity of mechanosensitive channels is also regulated by the mass of amyloplast and the protoplast, which exert a mechanical impact on the cell membrane (Garrill et al. 1996). Normally, thus gravisusceptores regulates growth and cell wall properties (Garrill et al. 1996; Soga et al. 2004) but the mass of amyloplast and therefore the mass of starch might also influence mechanosensitive channel opening in tuber cells if the tubers are exposed to mechanical impact.

\section{Conclusion}

Given cultivars displayed a range of tubers differing in their starch concentration. Variations of the starch concentration within one cultivar, as shown in the distribution of tubers in different specific gravity ranges, might have more effect on blackspot bruising then variations in the starch concentration between cultivars, which corresponded e.g. to results of the cv. Gala compared to the cv. Renate very well. Blackspot bruise occurred if the related enzymatic reaction was able to proceed, which was possible when individual cell membranes of cell organelles were open and released components. Membrane opening might be realised by mechanosensitive channels. Channel opening (Ghazi et al. 1998) as well as blackspot formation passed, if impact exceeded individual stress thresholds (Baritelle and Hyde 2003). Independent of the availability of chemical components, which determine bruise colour, the resistance of the mechanosensitive channels to opening, depended on the cell mass determined particularly by the amount of starch molecules. Starch in the amyloplast loaded the cell weight and activated mechanosensitive channels and sensing might occur outside the plasma membrane as also described by Sack (1997). Tubers

with higher starch concentration are less resistant to blackspot bruise. Therefore the aim of a potato crop management should be among others the generation of uniform tubers with similar starch concentrations within each cultivar or plant.

\section{References}

Alvarez, M.D., D.E.J. Saunders, and J.F.V. Vincent. 2000. Fracture properties of stored fresh and osmotically manipulated apple tissue. European Food Research and Technology 211: 284-290.

Baritelle, A., and G.H. Hyde. 2003. Effect of the specific gravity and cultivar on the impact sensitivity of potatoes. Postharvest Biology and Technology 29: 279-286. 
Barkhausen, R. 1978. Ultrastructural changes in wounded plant storage tissue. In Biochemistry of Wounded Plant Tissues. ed, G. Kagel, 1-42. Berlin: Walter de Gruyser.

Bäuerle, G., G. Otterbach, K. Gierschner, and G. Baumann. 1977. Bestimmung des Polyuronidgehaltes und des Veresterungsgrades des Pektinanteiles in Handelspektinpräparaten, Apfelsäften und Apfelmaceraten. Deutsche Lebensmittel Rundschau 73: 281-286.

Braun, I. 1989. Einfluss von physiologischer Ausreife und Lagerung auf einige qualitätsbestimmende Inhaltsstoffe verschiedener Kartoffelsorten unter besonderer Berücksichtigung der Zellwandsubstanzen und ihre Bedeutung für die Verarbeitung zu Kartoffeltrockenprodukten. Diss. Göttingen.

Bush, M.S., M. Marry, I.M. Huxham, M.C. Jarvis, and M.C. Mc Cann. 2001. Developmental regulation of Pectic epidopses during potato tuberization. Planta 213:869-880.

Bussieres, P. 1993. Potential dry matter and water rates in the Tomato fruit in relationship to fruit size. Annals of Botany 72: 63-72.

Chen, C.S., and D.E. Ingber. 1999. Tensegrity and mechanoregulation: from skeleton to cytoskeleton. Osteoarthritis and Cartilage 7: 81-94.

Edgell, T., E.R. Brierley, and A.H. Cobb. 1998. An ultrastructural study of bruising in stored potato (Solanum tuberosum L.) tubers. Annals of applied Biology 132: 143150.

Garrill, A., G.P. Findlay, and S.D. Tyerman. 1996. Mechanosensitive ion channels. In Membranes: Specialized Functions in Plants, ed. M. Smallwood, J.P. Knox, D.J. Bowles, 247-260. Guildford: Biddles Ltd.

Ghazi, A., C. Berrier, B. Ajouz, and M. Besnard. 1998. Mechanosensitive ion channels and their mode of activation. Biochemistry 80: 357-362.

Guillotin, S.E. 2005. Studies on the intra- and intermolecular distributions of substituents in commercial pectins. Ph.D.thesis, Wageningen, Netherlands.

Haase, N.U. 2003/2004. Estimation of dry matter and starch concentration in potatoes by determination of under-water weight and near infrared spectroscopy. Potato Research 46: 117-127.

Haase, N.U. 2008. Healthy aspects of potatoes as part of the human diet. Potato Research 51: 239-258.

ICC Standards 2006. ICC-Standard No. 123/1. Standard methods of the International Association for Cereal Science and Technology, Vienna.

Keijbets, M. 2008. Potato processing for the consumer: developments and further challenges. Potato Research 51: 271-281. 
Keijbets, M.J.H., and W. Pilnik. 1974. Some problems in the analysis of pectin in potato tuber tissue. Potato Research 17: 169-177.

Kohorn, B.D. 2000. Plasma membrane-cell wall contacts. Plant Physiology 124: 31-38.

Kolbe, H., and S. Stephan-Beckmann. 1997. Development, growth and chemical composition of the potato crop (Solanum tuberosum L.). II. Tuber and whole plant. Potato Research 40: 135-153.

Laboski, C.A.M., and K.A. Kelling. 2007. Influence of fertilizer management and soil fertility on tuber specific gravity: a review. American Journal of Potato Research 84: 283-290.

Lærke, P.E. 2001. Blackspot bruise in potato tubers. Denmark: The Royal Veterinary and Agricultural University.

Längere, J., G. Otterbach, G. Baumann, and K. Gierschner. 1978. Probleme bei der Anwendung der Cuprizonmethode zur Bestimmung des Poly-Uronidgehaltes und des Veresterungsgrades in Handelspektin. III. Mitteilung. Deutsche Lebensmittel Rundschau 74: 259-262.

Larcher, W. 1994. Pflanzen unter Stress. Ökophysiologie der Pflanzen. Stuttgart: Eugen Ulmer GmbH \& Co.

Moledina, K.H., M. Haydar, B. Ooraikul, and D. Hadziyev. 1981. Pectin changes in pre cooking step of dehydrated mashed potato production. Journal of Science of Food and Agriculture 32: 1091-1102.

Naumann, K., and R. Bassler. 1976. Handbuch der Landwirtschaftlichen Versuchs- und Untersuchungsmethodik. Die chemische Untersuchung von Futtermitteln. Melsungen: J. Neumann-Neudamm Verlag.

Pardede, E. 2004. A study on effect of calcium-magnesium-phosphorus fertilizer on potato tubers (Solanum tuberosum L.) and on physiochemical properties of potato flour during storage. Göttingen: Cuvillier Press.

Putz, B. 1998. Kartoffeln: Züchtung, Anbau, Verwertung. Hamburg: Behr's.

Reeve, R.M. 1968. Preliminary histological observation on internal blackspot in potatoes. American Potato Journal 45: 157-167.

Sack, F.D. 1997. Plastids and gravitropic sensing. Planta 203: 63-68.

Scanlon, M.G., C.H. Pang, and C.G. Biliaderis. 1996. The effect of osmotic adjustment on the mechanical properties of potato parenchyma. Food Research International 29: 481-488. 
Soga, K., K. Wakabayashi, S. Kamisaka, and T. Hoson. 2004. Graviperception in growth inhibition of plant shoots under hypergravity conditions produced by centrifugation is independent of that in gravitropism and may involve mechanoreceptors. Planta 218: 1054-1061.

Storey, M. 2007. The harvested crop. In: Potato biology and biotechnology. Advances and perspectives, ed. D. Vreugdenhil, 441-470. Amsterdam: Elsevier.

Struik, P.C., D. Vreugdenhil, A.J. Haverkort, C.B. Bus, and R. Dankert. 1991. Possible mechanisms of size and hierarchy among tubers on one stem of a potato (Solanum tuberosum L.) plant. Potato Research 34: 187-203.

Taylor, M.A., G.J. McDougall, and D. Stewart. 2007. Potato flavour and texture. In: Potato biology and biotechnology. Advances and perspectives, ed. D. Vreugdenhil, 525532. Amsterdam: Elsevier.

Tibensky, V., J. Rosik, and V. Zitko. 1963. Zur Bestimmung des Veresterungsgrades von Pektin. Nahrung 7: 312-325.

van Eck, H.J. 2007. Genetics of morphological and tuber traits. In: Potato biology and biotechnology. Advances and perspectives, ed. D. Vreugdenhil, 91-115. Amsterdam: Elsevier.

van Loon, C.D., and W. van den Berg. 2003/2004. The effect of chloride fertilization on blackspot susceptibility and other quality characteristics and on yield of potato. Potato Research 46: 147-154.

Wang, N., J.P. Butler, and D.E. Ingber. 1993. Mechanotransduction across the cell surface and through the cytoskeleton. Science 260: 1124-1127.

Wright, P.J., C.M. Triggs, and J.A.D. Anderson. 2005. Effects of specific gravity and cultivar on susceptibility of potato (Solanum tuberosum) tubers to blackspot bruising and bacterial soft rot. New Zealand Journal of Crop and Horticultural Science 33: 353361.

Wulkow, A., W.B. Herppich, and E. Pawelzik. 2009. Water relations in potato (Solanum tuberosum) tubers in comparison to their blackspot susceptibility (in preparation).

Yahraus, T., S. Chandra, L. Legendre, and P.S. Low. 1995. Evidence for mechanically induced oxidative burst. Plant Physiology 109: 1259-1266. 


\section{Zusammenfassende Diskussion}

Die Neigung der Kartoffelknollen zu Schwarzfleckigkeit kann mittels Schwarzfleckigkeitsindex bestimmt und berechnet werden und ist abhängig von einer Vielzahl von Faktoren, die gruppiert werden können, um deren Einfluss auf die Entstehung des Qualitätsmangels zu beschreiben. McGarry et al. (1996) fassten Faktoren zusammen, welche die Art der Entstehung der Schwarzfleckigkeit beeinflussen, Sortenmerkmale beschreiben und physikalisch, physiologisch und biochemisch wirken. Lærke (2001) beschrieb Faktoren, die entweder einen anatomischen und/oder physikalischen sowie einen physiologischen Einfluss ausüben. Wirsing (2001) führte die Stabilität sowie die Empfindlichkeit der Sorten gegenüber Schwarzfleckigkeit als wichtige Bezugspunkte an, die eine unterschiedliche Neigung zu Schwarzfleckigkeit bedingen. Die vorliegende Arbeit beschreibt, bezogen auf Wirsing (2001), Einflussfaktoren auf die Schwarzfleckigkeitsstabilität und -empfindlichkeit.

Die Stabilität bezeichnet das Verhältnis zwischen der Energie der einwirkenden mechanischen Belastung und dem Ausmaß der entstandenen Schwarzfleckigkeit (van Eck 2007) unter Berücksichtigung der mechanischen Eigenschaften des Knollengewebes, die von der spezifischen Dichte der Knollen bestimmt werden (Scanlon et al. 1996, Baritelle und Hyde 2003). Die spezifische Dichte gilt als sortentypisches Merkmal (Laboski und Kelling 2007) und beschreibt indirekt die Konzentration der Trockenmasse in den Knollen (Haase 2003/4). Die Bezeichnung Trockenmasse umfasst alle nicht-wässrigen Bestandteile der Knolle (Putz 1998) und beinhaltet zu etwa 80 \% Stärke, 10 \% zytoplasmatische Bestandteile und $10 \%$ Zellwandmaterial (Lærke 2001).

In einer Vielzahl von Studien wird ein wesentlicher Einfluss der Trockenmasse (u. a. Kunkel und Gardner 1965, Murphy und Gover 1966, Kolbe und Haase 1997, Workman und Holm 1984) auf die Neigung zu Schwarzfleckigkeit vermutet. Jedoch wird in anderen Arbeiten ein enger Zusammenhang zwischen der Stärkekonzentration und der Neigung zu Schwarzfleckigkeit für möglich gehalten (u. a. Scanlon et al. 1996, Lærke 2001, Baritelle und Hyde 2003). Ergebnisse der vorliegenden Arbeit zeigen, dass die Stärkekonzentration der Knollen maßgeblich deren Trockenmasse und somit die spezifische Dichte der Knollen und damit deren Neigung zu Schwarzfleckigkeit beeinflusst. Eine Stärkekonzentration von $140 \mathrm{~g} \mathrm{~kg}^{-1}$ FM und eine entsprechende Trockenmassekonzentration von $\geq 200 \mathrm{~g} \mathrm{~kg} \mathrm{FM}^{-1}$ führen zu einer geringen Neigung zu Schwarzfleckigkeit, die sich mit zunehmender Stärkekonzentration in den Knollen erhöht, unabhängig von Erntezeitpunkt, Sortenmaterial und Lagerbedingungen. Es wird vermutet, dass die Konzentration der Stärke das Gewicht der Zellen und die Aktivität mechanosensitiver Kanäle beeinflusst, so dass diese Kanäle nach mechanischer Belastung geöffnet werden können. Infolgedessen kann sich die intrazellulare Kompartmentierung ändern.

Die übrigen Bestandteile der Trockenmasse umfassen Zellwandmaterialien und zytoplasmatische Bestandteile. Letztere beinhalten Zytoskelettkomponenten, umschlossen von einer Plasmamembran sowie organische und anorganische Substanzen, die intrazelluläre, 
lokal begrenzte Stoffwechselprozesse ermöglichen, ohne dass das gesamte Gewebe beeinflusst wird (Buselmaier 2007). Somit kann eine teilweise Verfärbung des Gewebes erfolgen. Obwohl Schwarzfleckigkeit nicht mit äußeren sichtbaren Gewebeverletzungen verbunden ist, kann der Zusammenhalt und die Festigkeit der Zellwand die Wirkung mechanischer Stöße und daraus resultierende Gewebeverformungen beeinflussen (Alvarez et al. 2000, Storey 2007). Zellwandkohäsion und -festigkeit sind abhängig von der Zellwandstruktur (Taylor 2007) und Pektinkonzentration (Keijbets und Pilnik 1974) sowie dem Grad der Pektinveresterung, der die physiologischen Eigenschaften des Pektins bestimmt (Guillotin 2005). Die Konzentration übriger Trockenmassebestandteile in der Knolle beeinflusst u. a die Qualität von Kartoffelprodukten (Braun 1989, Moledina et al. 1981, van Eck 2007), jedoch nach den vorliegenden Ergebnissen nicht die unterschiedliche Neigung zu Schwarzfleckigkeit roher Knollen (s. Kapitel 5).

Die Steifheit der Zellwand wird durch deren Binnendruck (Turgor) beeinflusst (Alvarez et al. 2000, Dejmek and Miyawaki 2002). Der Einfluss des Turgors auf die Neigung zu Schwarzfleckigkeit wird bereits von McGarry et al. (1996) als unklar beschrieben, was in der Folge durch andere Arbeiten ergänzt werden kann. Die vorliegenden Ergebnisse entsprechen denen von Landrigan et al. (1996) und Bajema et al. (1998) und zeigen, dass turgeszentes Gewebe weniger zu Schwarzfleckigkeit neigt. Dagegen vermuten Baritelle und Hyde (2001), dass turgeszentes Gewebe eine verringerte Stabilität gegenüber mechanischen Belastungen besitzt und Lærke (2001) zeigt einen sortenabhängigen Einfluss des Turgors auf die Neigung zu Schwarzfleckigkeit (s. Kapitel 3).

Neben den Zellwandeigenschaften können ebenfalls die Zellmembraneigenschaften die Stabilität gegenüber Schwarzfleckigkeit beeinflussen. In einigen Arbeiten wird angenommen, dass eine Zerstörung der Zellmembran zur Entstehung von Schwarzfleckigkeit beiträgt (van Loon und van den Berg 2003/4, van Eck 2007, Storey 2007, Lulai 2007). Andere Autoren (Reeve 1968, Barkhausen 1978, Edgell et al. 1998) zeigen, dass die Zellmembran nicht zerstört werden muss, damit Melanin gebildet werden kann. Insofern scheint die Integrität der Zellmembran deren mechanische Belastbarkeit zu bestimmen, wie bereits von Scanlon et al. (1996) und Dejmek und Miyawaki (2002) beschrieben. Die Membranintegrität wird teilweise durch ihre Phosphorkonzentration (Bergmann 1993, Bucher und Kossmann 2007) beeinflusst, die in direkter Wechselwirkung mit der Phosphorverfügbarkeit des Bodens steht (Jacobsen et al. 1998). In den hier beschriebenen Versuchen sind die Knollen ausreichend mit Phosphor versorgt, wobei die Phosphorkonzentration keinen Einfluss auf die unterschiedliche Neigung zu Schwarzfleckigkeit hat. Die Stabilität vom Plasma- und Endomembranen wird außerdem von Kalzium beeinflusst (Sowokinos 2007). Kalziummangel kann die Membranfunktionen beeinträchtigen und schrittweise zur Zerstörung subzellulärer Kompartmentierung führen (Tzeng et al. 1986), begleitet von einem Anstieg unkontrollierter Oxidationsprozesse (Davies 1998, Sowokinos 2007). Eine zusätzliche Kalziumdüngung während der Vegetation erhöht den Kalziumgehalt in den Knollen und verringert potenziell das Auftreten innerer physiologischer Mängel (Simmons und 
Kelling 1987) wie Eisenfleckigkeit (Tzeng et al. 1986, Bartz et al. 1992), Braunherzigkeit (Davies 1998, Sowokinos 2007) und Hohlherzigkeit (Sowokinos 2007). Ebenso ist ein möglicher Zusammenhang zwischen dem Kalziumgehalt der Knollen und deren Anfälligkeit für Schwarzbeinigkeit (Tzeng et al. 1986) und Schwarzfleckigkeit (Lærke 2001) erwähnt. Dieser Zusammenhang kann mit den vorliegenden Ergebnissen nicht unterstützt werden, obwohl Kalzium in dreifach höherer Konzentration in den Knollen enthalten ist, als u. a. von Putz (1998) und Dilworth et al. (2007) als Durchschnittswert genannt. Da hohe Kalziumkonzentrationen die Boraufnahme verzögern, ist in den Knollen potentiell mit Bormangel zu rechnen (Bergmann 2003). Bormangel reduziert Bindungsmöglichkeiten für Kalzium an den Zellmembranen und erhöht die Konzentration freien Kalziums und Kaliums im Zytoplasma, wobei sich die Integrität der Zellmembran verringert (Mühling et al. 1998, Wang et al. 2003, Camacho-Christóbal et al. 2008). Die Borkonzentration in den untersuchten Knollen entspricht jedoch einer guten Nährstoffversorgung (Davies 1998) und korreliert nicht mit der unterschiedlichen Neigung zu Schwarzfleckigkeit. Dagegen sind Nährstoffverhältnisse (Bor war partiell involviert) teilweise mit der Neigung zu Schwarzfleckigkeit korreliert, die u. a. eine Aktivierung verschiedener Akzeptormoleküle der Zellmembran beeinflussen können (Marschner 2003). Obwohl Kalzium und Bor für die Kartoffel essentielle Nährstoffe sind (White et al. 2007), ist bisher nichts über das Kalzium/Bor-Verhältnis bekannt, das für eine optimale Entwicklung von Pflanze und Knolle notwendig ist. Bei Tomatenpflanzen (Solanum lycopersicum) ist ein Kalzium/BorVerhältnis zwischen 201 und 593 für eine normale Pflanzenentwicklung erforderlich. Bei Sellerie (Apium graveolens) ist bereits ein Kalzium/Bor-Verhältnis zwischen 15 und 100 für ein optimales Wachstum ausreichend, wobei ein Kalzium/Bor-Verhältnis über 100 relativen Bormangel signalisiert. Ähnliche Kalzium/Bor-Verhältnisse sind für Raps (Brassica napus) beschrieben (Bergmann 1993). Wechselwirkungen von Phosphor und Bor auf Grund ähnlicher Funktionen in der Zellmembran bleiben spekulativ (Bergmann 1993) (s. Kapitel 4).

Die Empfindlichkeit von Kartoffelknollen für Schwarzfleckigkeit beschreibt die tatsächlich auftretende Schwarzfleckigkeit (van Eck 2007) nach Überschreitung (Lærke 2001) einer bestimmten Belastungsstärke und/oder einer Anzahl von Stößen (Leppack 2001). Erst nach Abnahme der subzellularen Integrität erhöht sich somit das biochemische Potential zur Bildung von Melanin (Dean et al. 1993). Die Bildung von Melanin setzt die Anwesenheit von molekularem Sauerstoff (Edgell et al. 1998) oder das biochemische Potential der Knolle, freie Sauerstoffradikale zu bilden, voraus (Storey 2007). Nach Bakalova et al. (2004) sind freie Sauerstoffradikale bis zu einer gewissen Konzentration immer in der Knolle enthalten und bedingen die gute Durchlüftung des Gewebes (Lærke et al. 2002). Unter normalen (stressfreien) Bedingungen wird die Produktion und Zerstörung freier Sauerstoffradikale durch den Zellstoffwechsel reguliert (Bakalova et al. 2004). Durch mechanische Stöße erhöht sich der Gehalt freier Sauerstoffradikale (Yahraus 1995) und kann in den Zellen oxidativen Stress auslösen, der durch ein antioxidatives Abwehrsystem be- 
grenzt werden kann (Lærke et al. 2002). Die Wirkung antioxidativer Abwehrsysteme wird durch verschiedene Analysemethoden (Tab. 6.1) ermittelt. In den vorliegenden Untersuchungen werden Methoden verwendet, die eisen-reduzierende (FRAP) und hydroxylradikal-abwehrende (H-ORAC) Aktivitäten des antioxidativen Abwehrsystems bestimmen. Es ist jedoch nicht möglich, mit diesen Methoden die Hemmung der Bildung freier Sauerstoffradikale zu messen, welche zur Melaninsynthese genutzt werden können (Storey 2007). FRAP- und H-ORAC- Prozesse des antioxidativen Abwehrsystems erfolgen, ohne die Melaninsynthese deutlich zu beeinträchtigen und bilden daher keine methodische Grundlage, die Neigung zu Schwarzfleckigkeit spektrophotometrisch zu ermitteln (s. Kapitel 2). Kartoffeln enthalten eine Vielzahl leistungsfähiger Antioxidantien. Dazu zählen eine Reihe niedermolekularer Verbindungen wie Flavonoide (u. a. Flavonole, Kämpferol, Glutathion, Quercitin, Myricitin, Patatin), phenolische Verbindungen (Chlorogen-, Kumarin-, Ferula- und Kaffeesäure) und Vitamine (Blokhina 2003, Leo et al. 2008, Stushnoff et al. 2008). Von den Vitaminen ist vor allem Askorbinsäure antioxidativ wirksam (McNabnay et al. 1999, Pelegrini et al. 2003, Nzaramba et al. 2007). Die Konzentrationen von Askorbinsäure und Dehydroaskorbinsäure sowie Zitronensäure in Kartoffelknollen erhöhen sich bei optimaler Kalium- und Magnesiumversorgung (Rogozińska et al. 2005) und dadurch kann die Neigung zu Schwarzfleckigkeit vermindert werden (Heinecke 2007). Die Konzentrationen beider Nährstoffe entsprechen in der vorliegenden Untersuchung einer guten Versorgung und haben keinen Einfluss auf die unterschiedliche Neigung zu Schwarzfleckigkeit (s. Kapitel 4). Kartoffeln enthalten weiterhin verschiedene Enzyme, die den Gehalt freier Sauerstoffradikale verringern können. Hierzu zählen Superoxiddismutasen, Katalasen und Peroxidasen (Yahraus 1995, Blokhina et al. 2003, Leo et al. 2008) (s. Kapitel 2), wobei deren Aktivität u. a. durch Selen unterstützt werden kann. Obwohl Selen ein essentieller Nährstoff für Tiere, Bakterien und Algen, jedoch nicht für Pflanzen ist (McNeal und Balistrieri 1989, Terry et al. 2000), zeigen die Versuche von Seppänen et al. (2003) und Turakainen et al. (2006), dass der Gehalt freier Sauerstoffradikale in Blättern und Knollen von Kartoffelpflanzen durch zusätzliche Seleniumdüngung begrenzt werden kann, wobei sich ebenfalls die Qualität der Knollen verbessert. In den vorliegenden Untersuchungen hat im Gegensatz dazu die Selenkonzentration in den Knollen keinen Einfluss auf deren Neigung zu Schwarzfleckigkeit (s. Kapitel 4). 
Tabelle 6.1 Die Wirkung nicht-enzymatischer Antioxidantien in Kartoffeln und anderen pflanzlichen Produkten ermittelt unter Anwendung verschiedener Analysemethoden

\begin{tabular}{|c|c|c|c|c|c|}
\hline $\begin{array}{l}\text { anti- } \\
\text { oxidative } \\
\text { Substanz }\end{array}$ & Verbindung & Produkt & Analysemethode & Wirkung & Referenz \\
\hline \multirow[t]{2}{*}{ Phenole } & $\begin{array}{l}\text { Flavonole, } \\
\text { Flavone }\end{array}$ & $\begin{array}{l}\text { Kartoffel, } \\
\text { Heidelbeere, } \\
\text { Buchweizen- } \\
\text { sprossen, } \\
\text { Apfel }\end{array}$ & $\begin{array}{l}\text { ORAC, } \\
\text { Superoxid anion } \\
\text { scavenging- } \\
\text { Aktivität, } \\
\text { FRAP, } \\
\text { Trolox }\end{array}$ & $\begin{array}{l}\text { Elektronenakzeptor } \\
\text { Elektronendonator }\end{array}$ & $\begin{array}{l}\text { Wang et al. 2008, } \\
\text { Liu et al. 2008, } \\
\text { Sun-Waterhouse et } \\
\text { al. 2008, } \\
\text { Reddivari et al. } 2007\end{array}$ \\
\hline & Gesamtgehalt & $\begin{array}{l}\text { Kartoffel, } \\
\text { Getränke, } \\
\text { Heidelbeere, } \\
\text { Buchweizenspros- } \\
\text { sen, } \\
\text { Buchweizen, } \\
\text { Reis, } \\
\text { Roter Wein, } \\
\text { Sojabohne, } \\
\text { Qinoa, } \\
\text { Amaranth, } \\
\text { Grapefruit }\end{array}$ & $\begin{array}{l}\text { FRAP, } \\
\text { HPLC, } \\
\text { Gallussäure- } \\
\text { äquivalent, } \\
\text { Chlorogensäure- } \\
\text { äquivalent }\end{array}$ & Elektronendonator & $\begin{array}{l}\text { Seeram et al. 2008, } \\
\text { Stushnoff et al. 2008, } \\
\text { Wang et al. 2008, } \\
\text { Lui et al. 2008, } \\
\text { Gorinstein et al. } \\
\text { 2008, } \\
\text { Reddivari et al. 2007, } \\
\text { Nzaramba et al. } \\
\text { 2007, } \\
\text { Leo et al. 2008, } \\
\text { Pellegrini et al. 2000, } \\
\text { Reyes et al. 2005, } \\
\text { Gorinstein et al. 2004 }\end{array}$ \\
\hline $\begin{array}{l}\text { Vitamin } \\
\text { E }\end{array}$ & $\begin{array}{l}\text { Vitamin E- } \\
\text { Homologe } \\
\text { (Trolox) }\end{array}$ & $\begin{array}{l}\text { Kartoffel, } \\
\text { Getränke }\end{array}$ & $\begin{array}{l}\text { ORAC, } \\
\text { TEAC, } \\
\text { DPPH }\end{array}$ & Elektronendonator & $\begin{array}{l}\text { Seeram et al. 2008, } \\
\text { Stushnoff et al. 2008, } \\
\text { Reyes et al. } 2005\end{array}$ \\
\hline $\begin{array}{l}\text { Vitamin } \\
\text { C }\end{array}$ & Askorbinsäure & $\begin{array}{l}\text { Kartoffel, } \\
\text { Apfel, } \\
\text { Erdbeere, } \\
\text { Tomate }\end{array}$ & $\begin{array}{l}\text { FRAP, } \\
\text { Trolox, } \\
\text { HPLC, } \\
\text { titrimetrisch }\end{array}$ & Elektronendonator & $\begin{array}{l}\text { Sun-Waterhouse et } \\
\text { al. 2008, } \\
\text { Stushnoff et al. 2008, } \\
\text { Keutgen \& Pawelzik } \\
\text { 2007, Leo et al.2008, } \\
\text { George et al. } 2004\end{array}$ \\
\hline $\begin{array}{l}\text { Gesamt- } \\
\text { gehalt }\end{array}$ & & $\begin{array}{l}\text { Kartoffel, } \\
\text { italienisches Ge- } \\
\text { müse, } \\
\text { Getränke, Öle, } \\
\text { Buchweizen, } \\
\text { Reis, } \\
\text { Roter Wein, } \\
\text { Sojabohne, } \\
\text { Qinoa, } \\
\text { Amaranth, } \\
\text { Erdbeere, } \\
\text { Tomate, } \\
\text { Lippenblütler } \\
\text { (Minze), } \\
\text { Grapefruit }\end{array}$ & $\begin{array}{l}\text { TRAP, } \\
\text { TEAC, } \\
\text { TOSC, } \\
\text { FRAP, } \\
\text { CUPRAC, } \\
\text { DPPH, ABTS, } \\
\text { ORAC, } \\
\text { H-ORAC }\end{array}$ & Elektronendonator & $\begin{array}{l}\text { Pellegrini et al. 2003, } \\
\text { Leo et al. 2008, } \\
\text { Gorinstein et al. } \\
2008, \\
\text { Keutgen \& Pawelzik } \\
2007, \\
\text { Nzaramba et al. } \\
2007, \\
\text { Wu et al. 2004, } \\
\text { George et al. 2004, } \\
\text { Szöllösi \& Szöllösi } \\
\text { Varga 2002, } \\
\text { Gorinstein et al. } 2004\end{array}$ \\
\hline Terpene & $\beta$ - Karotinoid & Kartoffel & $\begin{array}{l}\text { spektro- } \\
\text { photometrisch }\end{array}$ & $\begin{array}{l}\text { Elektronenakzeptor } \\
\text { Elektronendonator }\end{array}$ & Leo et al. 2008 \\
\hline
\end{tabular}


Eine unkontrollierte Zunahme des Sauerstoffgehaltes ermöglicht eine spontane Oxidation und Polymerisation von Substraten, katalysiert durch Polyphenoloxidase, wobei Dopaquinon als Vorstufe des Melanins gebildet wird (Dean et al. 1993).

Substrate, die während der Melaninsynthese oxidiert werden, sind u. a. freies Tyrosin, Cystein, Chlorogen- und Kaffeesäure (Dean et al. 1993, Kirk et al. 2006). Die Substratkonzentration kann durch die Konzentration verschiedener Nährstoffe beeinflusst werden. Beispielsweise können eine optimale Kalium- (McNabnay et al. 1999) sowie Selenversorgung (Munshi et al. 1990, Turakainen et al. 2006) die Konzentration freier Aminosäuren (u. a. Tyrosin, Cystein) verringern, da Kalium an der Proteinsynthese beteiligt ist (McNabnay et al. 1999). Dagegen kann Eisenmangel die Konzentration freier Aminosäuren erhöhen sowie die Konzentration einzelner organischer Säuren ändern (Bergmann 1993) und somit möglicherweise die Neigung zu Schwarzfleckigkeit beeinflussen. Während des Kochprozesses ist Eisen ebenso in der Lage, Komplexe mit phenolischen Substanzen (Chlorogensäure) in der Knolle zu bilden und damit die Kochdunklung, einen durch nichtenzymatische Verfärbung hervorgerufenen Qualitätsmangel, zu fördern (Muneta und Kaisaki 1983, Griffith und Bain 1997). Phenolische Verbindungen (Chlorogen- und Kaffeesäure) können ebenfalls an der Melaninbildung beteiligt sein. Deren Konzentration erhöht sich u. a. bei Bormangel, wobei eine Bildung von 6-P-Gluconat-Borat-Komplexen mit Kaffeesäure verhindert wird (Mondy und Munshi 1993), die zu einer erhöhten Zufuhr der Substrate in den Pentose-Phosphat-Zyklus führt und damit die Phenolbildung fördert (Marschner 2003). Dagegen wird die Synthese von Hemicellulose und anderen Zellwandbestandteilen gehemmt (Mondy und Munshi 1993). Die Literaturhinweise zeigen, dass unzureichende Kalium-, Selen-, Eisen- und Borkonzentrationen in der Knolle die Konzentrationen verschiedener Verbindungen des Primär- und Sekundärstoffwechsels erhöhen und somit die Melaninsynthese fördern können. Die vorliegenden Ergebnisse belegen eine gute Nährstoffversorgung der Knollen, bei denen Kalium, Selen, Eisen und Bor ausreichend konzentriert sind und keinen Einfluss auf die Neigung zu Schwarzfleckigkeit haben (s. Kapitel 4). Die fortlaufende nicht-enzymatische Polymerisation von Dopaquinon mit nucleophilen Aminosäureresten von Proteinen zum stickstoffhaltigen Melanin erfolgt unabhängig von der Nährstoffkonzentration der Knollen.

Das Pigment Melanin bildet zwar den Qualitätsmangel Schwarzfleckigkeit aus, wirkt aber ebenfalls antioxidativ. Deren phenolische Komponenten nehmen freie Sauerstoffradikale auf und verhindern eine Ausdehnung der Kettenreaktion bei einer Oxidation mehrfach ungesättigter Fettsäuren in der Zellmembran nach mechanischem Stress. Somit verhindert oder verlangsamt die Melaninbildung eine Nekrosenbildung im Gewebe. Außerdem können die Quinonkomponenten des Melanins den Grad der Oxidation von ß-Karotinoiden verringern (Hung et al. 2002) und tragen somit zur Erhaltung der Konzentration weiterer antioxidativ wirkender Substanzen bei. 


\section{Schlussfolgerungen}

Die vorliegenden Ergebnisse zeigen, dass eine Optimierung der Anbau-, Nachernte- und Lagerbedingungen für Kartoffeln deren Stabilität gegenüber Schwarzfleckigkeit erhöhen kann. Die Stabilität gegenüber Schwarzfleckigkeit wird maßgeblich beeinflusst durch das Verteilungsspektrum von Knollen verschiedener spezifischer Dichten innerhalb einer Sorte und Pflanze. Dabei ist die Bildung von Knollen verschiedener spezifischer Dichten abhängig von der Verteilung und Akkumulation von Trockenmasse und Wasser in den Knollen (Bussieres et al. 1993, Kolbe und Stephan-Beckmann 1997, Struik et al. 1991). Die Trockenmassebildung kann teilweise durch ackerbauliche Maßnahmen beeinflusst werden. Nach Struik et al. (1990) ist die Konkurrenz der Knollen um Reservestoffe abhängig von der Anzahl knollenbildender Stängel und deren Konkurrenz um Reservestoffe. Dies betrifft sowohl Haupt- als auch Nebenstängel. Der Hauptstängel wird direkt von der Pflanzknolle gebildet. Dessen Entwicklung ist vom physiologischen Alter der Pflanzknolle (reguliert durch die Vegetationsverhältnisse während des Wachstums und Lagerungsbedingungen) und deren Größe, Form, Genotyp, Vorkeimungen und Keimschädigungen während des Legens abhängig und wird durch Umweltbedingungen nach dem Legen (Feuchte, Temperatur, Bodenstruktur) beeinflusst. Die Nebenstängel, als unterirdische Verzweigungen des Hauptstängels, können durch die Pflanzdichte, Stickstoffdüngung sowie durch die Größe und das physiologische Alter der Pflanzknolle beeinflusst werden. Eine relativ gleichmäßige Knollenentwicklung wird $\mathrm{u}$. a. durch gleichmäßige Pflanzabstände und durch Pflanzknollen gleicher Größensortierung und physiologischen Alters bei optimaler Bestandesführung gefördert. Dabei sollte eine optimale Bestandesführung eine zusätzliche bedarfsorientierte Beregung beinhalten, die neben einer ausreichenden Nährstoffzufuhr eine ausreichende Konzentration von Wasser in den Knollen ermöglicht, um deren Stabilität gegenüber Schwarzfleckigkeit zu erhöhen. Außerdem vermindert eine ertragsorientierte Düngung Nährstoffmangel bei Kartoffeln und damit verbundene Qualitätsverluste, wobei außerordentliche Düngergaben nicht qualitativ wirksam würden.

Dagegen kann die Empfindlichkeit für Schwarzfleckigkeit, verursacht durch die Konzentration schwarzfleckigkeitsförderner oder -hemmender Substanzen, möglicherweise durch züchterische Bearbeitung beeinflusst werden. Eine Beeinflussung der Konzentration antioxidativ wirkender Substanzen kann die Neigung zu Schwarzfleckigkeit verändern, wobei übermäßig produzierte Sauerstoffradikale durch Elektronenakzeptoren (u. a. Flavonole, Flavone, ß-Karotinoide) gebunden werden können, bevor diese für die Melaninsynthese nutzbar sind. Grundsätzlich gilt bei der züchterischen Beeinflussung der Neigung zu Schwarzfleckigkeit zu bedenken, dass die Bildung von Melanin ein Ergebnis des mechanischen Stresses sein kann. Jedoch kann Melanin auch antioxidativ wirken, weil es freie Sauerstoffradikale aufnimmt, deren Konzentration nicht durch andere antioxidative Abwehrsysteme der Knolle verringert wird. 


\section{Zusammenfassung}

Melanin ist ein Pigment, welches in der Rindenschicht von Kartoffelknollen gebildet wird und ein bis mehrere Gewebeabschnitte grau bis schwarz erscheinen lässt. Diese Verfärbung des Knollengewebes wird als Schwarzfleckigkeit bezeichnet und stellt einen inneren Qualitätsmangel dar, der äußerlich nur schwer zu erkennen ist (Radke et al. 2000). Mit dem Auftreten von Schwarzfleckigkeit können erhebliche finanzielle Verluste auf allen Stufen der Wertschöpfungskette verbunden sein. Dieses Problem war bereits Gegenstand einer Vielzahl von wissenschaftlichen Untersuchungen und die vorliegende Arbeit baut darauf mit speziellem Fokus auf physiologische Vorgänge in der Knolle auf.

Grundsätzlich zeigte sich, dass Knollen verschiedener Sorten sowie Knollen einer Sorte in unterschiedlicher Intensität zu Schwarzfleckigkeit neigten. Außerdem waren Knollen, die zwei Wochen vor dem üblichen Erntetermin gerodet wurden, weniger schwarzfleckig, als Knollen, die zum üblichen Erntezeitpunkt gerodet worden waren. Daher schienen mehrere Faktoren am Entstehen dieses Qualitätsmangels beteiligt zu sein.

Um den Einfluss der Jahreswitterung zu quantifizieren, wurden die Kartoffeln über drei Vegetationsperioden angebaut. Die Ergebnisse zeigten, dass Sonnenscheindauer, Niederschlagsmenge (inklusive Beregnung) und die durchschnittliche Temperatur im Untersuchungszeitraum über alle drei Jahre nahezu gleich waren und keinen Einfluss auf die unterschiedliche Neigung zu Schwarzfleckigkeit hatten. Die Neigung zu Schwarzfleckigkeit ist u. a. sortenabhängig und wurde daher an acht verschiedenen, überregional bedeutenden Speisekartoffeln untersucht, die den Qualitätsmangel unterschiedlich ausprägten. Ein Vergleich der Sorten wurde ermöglicht, indem Knollen gleicher Größen im Bereich von 40 bis $50 \mathrm{~mm}$ untersucht wurden, die zusätzlich nach ihren spezifischen Dichten getrennt wurden. Alle Sorten neigten nach der dritten Vegetationsperiode signifikant höher zu Schwarzfleckigkeit, als nach den vorherigen Vegetationsperioden. Der Anbau der Sorten erfolgte in einer kartoffelintensiven Anbauregion unter praxisüblichen Bedingungen, die u. a. eine bedarfsorientierte Beregung und Nährstoffversorgung einschlossen. Die Nährstoffkonzentrationen von Kalium, Phosphor, Kalzium, Magnesium, Selen, Bor und Eisen wurden untersucht, da sie verschiedene Funktionen im Zellstoffwechsel besitzen, die u. a. in Beziehung zur Neigung zu Schwarzfleckigkeit stehen. Dabei zeigten die vorliegenden Ergebnisse, dass die praxisübliche Erhaltungsdüngung ausreichend war, um die Knollen gut mit Nährstoffen zu versorgen, wobei deren Konzentrationen in den Knollen keinen Einfluss auf die unterschiedliche Neigung zu Schwarzfleckigkeit hatten.

Es wurde deutlich, dass sich in geernteten Knollen der Stoffwechsel verlangsamte, indem u. a. der Stärkeabbau eingeschränkt war. Dadurch verringerte sich die Transpiration der Knollen, wobei deren Konzentration an Wasser erhalten blieb. Während der anschließenden Lagerung bei $4^{\circ} \mathrm{C}$ für fünf und acht Monate blieben die Knollen turgeszent, hervorgerufen durch ihre osmotische Anpassung an niedrige Temperaturen und unterstützt durch eine hohe relative Luftfeuchte von 95 \%. Die Neigung zu Schwarzfleckigkeit veränderte 
sich während der Lagerung nach allen drei Vegetationsperioden verschieden, unabhängig von der Wasserkonzentration in den Knollen. Jedoch beeinflusste die Konzentration der Trockenmasse in den Knollen deren Neigung zu Schwarzfleckigkeit. Von den untersuchten Trockenmassebestandteilen (Stärke, Zellwandbestandteile, zytoplasmatische Komponenten) korrelierte nur die Konzentration der Stärke mit der Neigung zu Schwarzfleckigkeit.

Anhand der vorliegenden Ergebnisse konnten Maßnahmen abgeleitet werden, die für die Kartoffelproduktion, -lagerung und -züchtung Möglichkeiten aufzeigen, die Neigung der Knollen zu Schwarzfleckigkeit zu vermindern. 


\section{Literatur zu den Kapiteln 1 und 6 bis 8}

Alvarez M.D., Saunders D.E.J., Vincent J.F.V. 2000: Effects of turgor pressure on the cutting energy of stored potato tissue. European Food Research and Technology 210, 331-339.

Bajema R.W., Hyde G.M., Baritelle A.L. 1998: Effects of mannitol on turgor and on failure stress and strain in potato tuber tissue. Journal of Postharvest Biology and Technology 14, 199-205.

Bakalova S., Nikolova A., Nedeva D. 2004: Isoenzyme profiles of peroxidase, catalase and superoxide dismutase as affected by dehydration stress and during germination of wheat seeds. Bulgarien Journal of Plant Physiology 30, 64-77.

Baritelle A.L., Hyde G.H. 2001: Commodity conditions to reduce impact bruising. Postharvest Biology and Technology 21, 331-339.

Baritelle A., Hyde G.H. 2003: Effect of the specific gravity and cultivar on the impact sensitivity of potatoes. Postharvest Biology and Technology 29, 279-286.

Barkhausen R. 1978: Ultrastructural changes in wounded plant storage tissue. In Biochemistry of Wounded Plant Tissues. Kagel G. (Ed), Walter de Gruyser, Berlin, pp. 1-42.

Bartz J.A., Locascio S.J., Weingartner D.P. 1992: Calcium and potassium fertilization of potatoes grown in North Florida. II: Effect on Bacterial Soft Rot potential in the tuber. American Potato Journal 69, 39-50.

Benzie I.F.F., Strain J.J. 1996: The ferric reducing ability of plasma (FRAP) as a measure of antioxidant power: The FRAP assay. Analytical Biochemistry 239, 70-76.

Bergmann W. 1993: Ernährungsstörungen bei Kulturpflanzen. Verlag Gustav Fischer Jena.

Blokhina O., Virolainen E., v. Fagerstedt K. 2003: Antioxidants, oxidative damage and oxygen deprivation stress: A review. Annals of Botany 91, 179-194.

Boumann A. 1995: Beschädigungen der Kartoffeln- Vom Roden bis zum Abpacken. Kartoffelbau 46, 242-244.

Braun I. 1989: Einfluss von physiologischer Ausreife und Lagerung auf einige qualitätsbestimmende Inhaltsstoffe verschiedener Kartoffelsorten unter besonderer Berücksichtigung der Zellwandsubstanzen und ihre Bedeutung für die Verarbeitung zu Kartoffeltrockenprodukten. Dissertation, Fachbereich Agrarwissenschaften, Georg-AugustUniversität Göttingen, Deutschland.

Bucher M., Kossmann J. 2007: Molecular physiology of the mineral nutrition of the potato. In: Potato biology and biotechnology. Advances and perspectives, Vreugendhil D. (Ed), 1. Elsevier, Amsterdam, pp. 441-470.

Buselmaier W. 2007: Biologie für Mediziner. Springer, Heidelberg. 
Bussieres P. 1993: Potential dry matter and water rates in the Tomato fruit in relationship to fruit size. Annals of Botany 72, 63-72.

Camacho-Christóbal J.J., Rexach J., Gonzáles-Fontes A. 2008: Boron in plants: Deficiency and toxicity. Journal of integrative Plant Biology 50, 1247-1255.

Corsini D., Stark J., Thornton M. 1999: Factors contributing to the blackspot bruise potential of Idaho Potato fields. American Journal of Potato Research 76, 221-226.

Davies H.V. 1998: Physiological mechanisms associated with the development of internal necrotic disorders of potato. American Journal of Potato Research 75, 37-44.

Davenport J.R., Milburn P.H., Rosen C.J., Thornton R.E. 2005: Environmental impacts of potato nutrient management. American Journal of Potato Research 82, 321-328.

Dean B.B., Jackowiack N., Nagle M., Pavek J., Corsini D. 1993: Blackspot pigment development of resistant and susceptible Solanum tuberosum L. genotypes at harvest and during storage measured by three methods of evaluation. American Potato Journal 70, 201-217.

Dejmek P., Miyawaki O. 2002: Relationship between the electrical and rheological properties of potato tuber tissue after various forms of processing. Journal of Bioscience, Biotechnology and Biochemistry 66, 1218-1223.

Delgado E., Poberezny J., Pawelzik E., Rogozińska I. 2001: Comparison of two methods for determining the discoloration potential of potato tubers based on their chemical and biochemical properties. American Journal of Potato Research 78, 389-394.

Dilworth L.L., Omoruyi F.O., Asemota H.N. 2007: In vitro availability of some essential minerals in commonly eaten processed and unprocessed Caribbean tuber crops. BioMetals 20, 37-42.

Edgell T., Brierley E.R., Cobb A.H. 1998: An ultrastructural study of bruising in stored potato (Solanum tuberosum L.) tubers. Annals of applied Biology 132, 143-150.

George B., Kaura C., Khurdiya D.S., Kapoor H.C. 2004: Antioxidant in tomato (Lycopersium esculentum) as a function of genotype. Food Chemistry 84, 45-51.

Gorinstein S., Zachwieja Z., Katrich E., Pawelzik E., Haruenkit R., Trakhtenberg S., Martin-Belloso O. 2004: Comparison of the contents of the main antioxidant compounds and the antioxidant activity of white grapefruit and his new hybrid. Lebensmittelwissenschaften und -Technologie 37, 337-343.

Gorinstein S., Lojek A., Číž M., Pawelzik E., Delgado-Licon E., Medina O.J., Moreno M., Salas I.A., Goshev I. 2008: Comparison of composition and antioxidant capacity of some cereals and pseudo cereals. International Journal of Food Science and Technology 43, 629-637. 
Griffiths D.W., Bain H. 1997: Photo-induced changes in the concentrations of individual chlorogenic acid isomers in potato (Solanum tuberosum) tubers and their complexation with ferric ions. Potato Research 40, 307-315.

Guillotin S.E. 2005: Studies on the intra-and intermolecular distributions of substituents in commercial pectins. Ph.D. thesis Universität Wageningen.

Hamester W. 1997: 100 Jahre Wandel im Handel. In: Speisefrischkartoffeln. Qualität erzeugen, erfassen, lagern vermarkten. Pötke E., Schumann P. (Ed), Agrimedia, Holm, pp. $117-122$.

Haase N.U. 2003/4: Estimation of dry matter and starch concentration in potatoes by determination of under-water weight and near infrared spectroscopy. Potato Research 46, 117-127.

Heinecke A. 2007: Beitrag zur Ermittlung der biochemischen Ursachen der Schwarzfleckigkeit bei Kartoffeln. Fachbereich Agrarwissenschaften, Georg-August-Universität Göttingen, Deutschland.

Hung Y.-C., Sava V.M., Makan S.Y., Chen T.-H.J., Hong M.-Y., Huang G.S. 2002: Antioxidant activity of melanins derived from tea: comparison between different oxidative states. Food Chemistry 78, 233-240.

Jacobsen H.B., Madsen M.H., Christiansen J., Nielsen T.H. 1998: The degree of starch phosphorylation as influenced by phosphate deprivation of potato (Solanum tuberosum L.) plants. Potato Research 41, 109-116.

Keijbets M.J.H., Pilnik W. 1974: Some problems in the analysis of pectin in potato tuber tissue. Potato Research 17, 169-177.

Keutgen A.J., Pawelzik E. 2007: Modifications of strawberry fruit antioxidant pools and fruit Quality under $\mathrm{NaCl}$ stress. Journal of Agriculture and Food Chemistry 55, 4066-4072.

Kirk W.W., da Rocha A.B., Hollosy S.I., Hammerschmidt R., Wharton P.S. 2006: Effect of soil salinity on internal browning of potato tuber tissue in two soil types. American Journal of Potato Research 83, 223-232.

Kolbe H., Haase N.U. 1997: Einflussfaktoren auf die Inhaltsstoffe der Kartoffel. Kartoffelbau 48, 234-240.

Kolbe H., Stephan-Beckmann S. 1997: Development, growth and chemical composition of the potato crop (Solanum tuberosum L.). II. Tuber and whole plant. Potato Research 40, 135-153.

Kunkel R., Gardner W.H. 1965: Potato tuber hydration and its effect on blackspot of Russet Burbank potatoes in the Columbia basin of Washington. American Potato Journal 42 , 109-124. 
Laboski C.A.M., Kelling K.A. 2007: Influence of fertilizer management and soil fertility on tuber specific gravity: A review. American Journal of Potato Research 84, 283-290.

Lærke P.E. 2001: Blackspot bruise in potato tubers: Ph.D. thesis, Department of Plant Biology, The Royal Veterinary and Agricultural University, Denmark.

Lærke P.E., Christiansen J., Veierskov B. 2002: Colour of blackspot bruises in potato tubers during growth and storage compared to their discolouration potential. Postharvest Biology and Technology 26, 99111.

Landrigan M., Morris S.C., Eamus D., McGlasson W.B. 1996: Postharvest water relationships and tissue browning of rambutan fruit. Scientia Horticulturae 66, 201-208.

Leo L., Leone A., Longo C., Lombardi D.A., Raimo F., Zacheo G. 2008: Antioxidant Compounds and Antioxidant Activity in "Early Potatoes". Journal of Agricultural and Food Chemistry 56, 41544163.

Leppack E. 2001: Messungen von Knollenbeanspruchungen und Schlussfolgerungen für die Verfahrensgestaltung. In: Wege zur Verbesserung der Kartoffelqualität durch Verminderung der mechanischen Beanspruchung. ATB/KLAS-Verband (Hrsg), Agrimedia, Bergen/Dumme, pp. 27-31.

Liu C-L., Chen Y-S., Yang J-H., Chiang B-H. 2008: Antioxidant activity of Tartary (Fagopyrum tataricum (L.) Gaert.) and common (Fagopyrum esculentum Moench) Buckwheat sprouts. Journal of Agriculture and Food Chemistry 56, 173-178.

Lulai, E.C. 2007: Skin-set, wound healing, and related defects. In: Potato biology and biotechnology. Advances and perspectives, Vreugendhil D. (Ed), 1. Elsevier, Amsterdam, pp. 471-500.

Marschner H. 2003: Mineral Nutrition of Higher Plants. Academic Press, London.

McGarry A., Hole C.C., Drew R.L.K., Parsons N. 1996: Internal damage in potato tubers: A critical review. Postharvest Biology and Technology 8, 239-258.

McNabnay M., Dean B.B., Bajema R.W., Hyde G.M. 1999: The effect of potassium deficiency on chemical, biochemical and physical factors commonly associated with blackspot development in potato tubers. American Journal of Potato Research 75, 5360.

McNeal J.M. and Balistrieri L.S. 1989: Geochemistry and Occurrence of selenium: An overview. In: Selenium in Agriculture and the environment, Heichel G.H., Kissel D.E., Ernst S. (Ed), SSSA Special Publication, 23, pp. 1-13.

Meier U. 2001: Entwicklungsstadien mono- und dikotyler Pflanzen. BBCH Monografie. Biologische Bundesanstalt für Land und Forstwirtschaft, Berlin und Braunschweig

Moledina K.H., Haydar M., Ooraikul B., Hadziyev D. 1981: Pectin changes in pre cooking stepp of dehydrated mashed potato production. Journal of Science of Food and Agriculture 32, 1091-1102. 
Mondy N.I., Munshi C.B., 1993: Effect of Boron on enzymatic discoloration and phenolics and ascorbic acid contents of potatoes. Journal of Agricultural and Food Chemistry 41, 554-556.

Mühling K.H., Wimmer M., Goldbach H.E. 1998: Apoplastic and membrane associated $\mathrm{Ca}^{2+}$ in leaves and roots as affected by boron deficiency. Plant Physiology 102, 179184.

Muneta P., Kaisaki F. 1983: Ascorbic acid-ferrous iron $\left(\mathrm{Fe}^{++}\right)$complexes and after cooking darkening of potatoes. American Potato Journal 62, 531-536.

Munshi C.B., Combs G.F., Mondy N.I. 1990: Effect of selenium treatment on the nitrogenous constituents of the potato. Journal of Agricultural and Food Chemistry 38, 2000-2002.

Murphy H.J., Gover M.J. 1966: The last decade in 38 years of potash studies for potato fertilizers in Maine. American Potato Journal 43, 122-127.

Nzaramba M.N., Bamberg J.B., Miller (Jr) J.C. 2007: Effect of propagule type and growing environment on antioxidant activity ant total phenolics content in potato germplasm. American Journal of Potato Research 84, 323-330.

Pavek J., Corsini D., Nissley F. 1985: A rapid method for determining blackspot susceptibility of potato clones. American Journal of Potato Research 62, 511-517.

Pawelzik E., Delgado E. 1999: Wirkung von Trockenstress auf die Verfärbungsneigung von Kartoffelknollen. Kartoffelbau 50, 358-360.

Pellegrini N., Serafini M., Colombi B., del Rio D., Salvatore S., Bianchi M., Brighenti F. 2003: Total antioxidant capacity of plant foods, beverages and oils consumed in Italy assessed by three different in vitro assays. American Society for Nutritional Science 133, 2812-2819.

Peters R. 1999: Qualitätskartoffel erzeugen, Beschädigungen vermeiden. Auswertungsund Informationsdienst für Ernährung, Landwirtschaft und Forsten (aid) e.V., Bonn.

Pötke E. 2001: Verfahrensvergleich zur Kartoffellagerung in loser Schüttung und in Behältern. In: Wege zur Verbesserung der Kartoffelqualität durch Verminderung der mechanischen Beanspruchung. ATB/KLAS-Verband (Hrsg.), Agrimedia, Bergen/Dumme, pp. 93-106.

Putz B. 1998: Kartoffeln: Züchtung, Anbau, Verwertung. Verlag Behr's, Hamburg.

Radtke W., Rieckmann W., Brendler F. 2000: Kartoffel- Krankheiten, Schädlinge, Unkräuter, Verlag Th. Mann, Gelsenkirchen.

Reddivari L., Hale A.L., Miller (Jr) J.C. 2007: Determination of phenolic content, composition and their contribution to antioxidant activity in specialty potato selections. American Journal of Potato Research 84, 275-282. 
Reeve R.M. 1968: Preliminary histological observation on internal blackspot in potatoes. American Potato Journal 45, 157-167.

Reyes L.F., Miller J.C., Cisneros-Zevallos L. 2005: Antioxidant capacity, anthocyanins and total phenolics in purple and red-fleshed potato (Solanum tuberosum L.) genotypes. American Journal of Potato Research 82, 271-277.

Rogozińska I., Wszelaczyńska E., Wichrowska D. 2005: Effect of bioelements (Mg, N, K) and herbicides on vitamin $\mathrm{C}$ content in potato tubers. Part I: Vitamin C content in potato tubers immediately after harvest. Journal of Elementology 10, 999-1008.

Sabba R.P., Dean B.B. 1996: Effect of cold storage on proteinase and chorismate mutase activities in Solanum tuberosum L. genotypes differing in blackspot susceptibility. American Potato Journal 73, 113-122.

Scanlon M.G., Pang C.H., Biliaderis C.G. 1996: The effect of osmotic adjustment on the mechanical properties of potato parenchyma. Food Research International 29, 481-488.

Schick R., Klinkowski M. 1961: Die Kartoffel. Ein Handbuch. 1., Deutscher Landwirtschaftsverlag, Berlin.

Schuhmann P. 1997: Veränderte Stellung der Kartoffel in der Landwirtschaft. In: Speisefrischkartoffeln. Qualität erzeugen, erfassen, lagern vermarkten. Pötke E, Schumann P. (Ed), Agrimedia, Holm, pp. 55-60.

Schuhmann P. 2001: Kartoffelbeschädigungen im Produktionsverfahren In: Wege zur Verbesserung der Kartoffelqualität durch Verminderung der mechanischen Beanspruchung. ATB/KLAS-Verband (Hrsg), Agrimedia, Bergen/Dumme, pp. 9-26.

Seeram N.P., Aviram M., Zhang Y., Henning S.M., Feng L., Dreher M., Heber D. 2008: Comparison of antioxidant potency of commonly consumed polyphenol-rich beverages in the United States. Journal of Agricultural and Food Chemistry 56, 1415-1422.

Seppänen M., Turakainen M., Hartikainen H. 2003: Selenium effects on oxidative stress in potato. Plant Science 165, 311-319.

Simmons K.E., Kelling K.A. 1987: Potato responses to calcium application on several soil types. American Potato Journal 64, 119-136.

Sowokinos J.R. 2007: Internal physiological disorders and nutritional and compositional factors that affect market quality. In: Potato biology and biotechnology. Advances and perspectives, Vreugdenhil D. (Ed), 1. Elsevier, Amsterdam, pp. 501-523.

Specht A. 1997: Qualitätsfördernde Erntetechnik und Ernteverfahren. In: Speisefrischkartoffeln. Qualität erzeugen, erfassen, lagern vermarkten. Pötke E., Schumann P. (Ed), Agrimedia, Holm, pp. 55-60.

Storey, M. 2007: The harvested crop. In: Potato biology and biotechnology. Advances and perspectives, Vreugendhil D. (Ed), 1. Elsevier, Amsterdam, pp. 441-470. 
Struik P.C., Vreugdenhil D., Haverkort A.J., Bus C.B., Dankert R. 1991: Possible mechanisms of size and hierarchy among tubers on one stem of a potato (Solanum tuberosum L.) plant. Potato Research 34, 187-203.

Stushnoff C., Holm D., Thompson M.D., Jiang W., Thompson H.J., Joyce N.I., Wilson P. 2008: Antioxidant properties of cultivars and selections from the Colorado potato breeding program. American Journal of Potato Research 85, 267-276.

Sun-Waterhouse D., Melton L.D., O'Connor C.J., Kilmartin P.A., Smith B.G. 2008: Effect of apple cell wall and their extracts on the activity of dietary antioxidants. Journal of Agricultural and Food Chemistry 56, 289-295.

Szöllösi R., Szöllösi Varga I. 2002: Total antioxidant power in some species of Labiatae (Adaptation of FRAP method). Acta Biologica Szegediensis 46, 125-127.

Taylor M.A., McDougall G.J., Stewart D. 2007: Potato flavour and texture. In: Potato biology and biotechnology. Advances and perspectives, Vreugdenhil D. (Ed), 1. Elsevier, Amsterdam, pp. 525-532.

Terry N., Zayed A.M., Souza M.P., Tarun A.S. 2000: Selenium in higher plants. Annual Review of Plant Physiology and Pant Molecular Biology 51, 401-432.

Thornton R.E., Timm H. 1990: Influence of fertilizer and irrigation management on tuber bruising. American Potato Journal 67, 45-54.

Thornton M., Bohl W. 2000: Preventing potato bruise damage. Educational Publication Warehouse, University of Idaho.

Turakainen M., Hartikainen H., Ekholm P., Seppänen M.M. 2006: Distribution of selenium in different biochemical fractions and raw darkening degree of potato (Solanum tuberosum L.) tubers supplemented with selenate. Journal of Agricultural and Food Chemistry $54,8617-8622$.

Tzeng K.C., Kelman A., Simmons K.E., Kelling K.A. 1986: Relationship of calcium nutrition to internal brown spot of potato tubers and sub-apical necrosis of sprouts. American Potato Journal 63, 87-97.

van Eck H.J. 2007: Genetics of morphological and tuber traits. In: Potato biology and biotechnology. Advances and perspectives, Vreugendhil D. (Ed), 1. Elsevier, Amsterdam, pp. 91-115.

van Loon C.D., van den Berg W. 2003/4: The effect of chloride fertilization on blackspot susceptibility and other quality characteristics and on yield of potato. Potato Research 46, 147-154.

Wang H.C. Du F.X., Yang Y. 2003: Effects of boron and calcium supply on calcium fractionation in plants and suspension cells of rape cultivars with different boron efficiency. Journal of Plant Nutrition 26, 789-806. 
Wang S.Y., Chen C-T., Sciarppa W., Wang C.Y., Camp M.J. 2008: Fruit quality, antioxidant capacity, and flavonoid content of organically and conventionally grown Blueberries. Journal of Agricultural and Food Chemistry 56, 5788-5794.

White P.J., Wheatley R.E., Hammomd J.P., Zhang K. 2007: Minerals, soils and roots In: Potato biology and biotechnology. Advances and perspectives, Vreugendhil D. (Ed), 1. Elsevier, Amsterdam, pp. 739-752.

Wirsing F. 2001: Erkenntnisse zum Auftreten und zur Vermeidung von Schwarzfleckigkeit. In: Wege zur Verbesserung der Kartoffelqualität durch Verminderung der mechanischen Beanspruchung. ATB/KLAS-Verband (Hrsg), Agrimedia, Bergen/Dumme, pp. $33-48$.

Workman M., Holm D.G. 1984: Potato clone variation in Blackspot and soft rot susceptibility, redox potential, ascorbic acid, dry matter and potassium. American Potato Journal $61,723-733$.

Wormanns G. 2001: Rechnergestützte Qualitätssicherung von Speisekartoffeln. In: Wege zur Verbesserung der Kartoffelqualität durch Verminderung der mechanischen Beanspruchung. ATB/KLAS- Verband (Hrsg), Agrimedia, Bergen/Dumme, pp. 49-58.

Wu X., Gu L., Holden J., Haytowitz D.B., Gebhardt S.E., Beecher G., Prior R.L. 2004: Development of a database for total antioxidant capacity in foods: a preliminary study. Journal of Food Composition and Analysis 17, 407-422.

Wu X., Beecher G.R., Holden J.M., Haytowitz D.B., Gebhardt S.E., Prior R.L. 2004: Lipophilic and hydrophilic antioxidant capacities of common foods in the United States. Journal of Agriculture and Food Chemistry 52, 4026-4037.

Yahraus T., Chandra S., Legendre L., Low P.S. 1995: Evidence for mechanically induced oxidative burst. Plant Physiology 109, 1259-1266. 


\section{Anhang}

Nachfolgend sind Einzel- oder Mittelwerte von Faktoren vermerkt, deren möglicher Einfluss auf die Neigung zu Schwarzfleckigkeit untersucht wurde. Signifikante Unterschiede zwischen den Werten sind dargestellt und beschrieben als:

A, B, C unterschiedliche Buchstaben kennzeichnen signifikante Unterschiede innerhalb eines Einflussfaktors zwischen den Jahren unabhängig von der Lagerung, Sorte und der spezifischen Dichte

A, B, C unterschiedliche Buchstaben kennzeichnen signifikante Unterschiede innerhalb eines Einflussfaktors zwischen den Stadien ${ }^{\S}$ innerhalb eines Jahres unabhängig von der Sorte und der spezifischen Dichte

a, b, c unterschiedliche Buchstaben kennzeichnen signifikante Unterschiede innerhalb eines Einflussfaktors zwischen den Sorten innerhalb eines Stadiums und eines Jahres unabhängig von der spezifischen Dichte

$a, b, c$ unterschiedliche Buchstaben kennzeichnen signifikante Unterschiede innerhalb eines Einflussfaktors zwischen den Stadien innerhalb einer Sorte und eines Jahres unabhängig von der spezifischen Dichte

a, b, c unterschiedliche Buchstaben kennzeichnen signifikante Unterschiede innerhalb eines Einflussfaktors zwischen den Dichten innerhalb eines Stadiums und eines Jahres unabhängig von der Sorte (außer in Tab. 10.43, 10.44, 10.45)

${ }^{\S}$ Stadium bezieht sich auf ein Zeitintervall, welches den Zeitabschnitt nach der Vorernte, Ernte oder nach fünf bzw. acht Monaten Lagerung eingrenzt 
Tabelle 10.1 Schwarzfleckigkeit [Mittelwert $(\bar{x})$ und Standartabweichung $\left(\sigma_{\mathrm{x}}\right)$ ] $(\%)$ in Sorten und Knollen verschiedener spezifischer Dichten $\left(\mathrm{kg} \mathrm{L}^{-1}\right)$ nach der Ernte 2005 und nach fünf und acht Monaten Lagerung

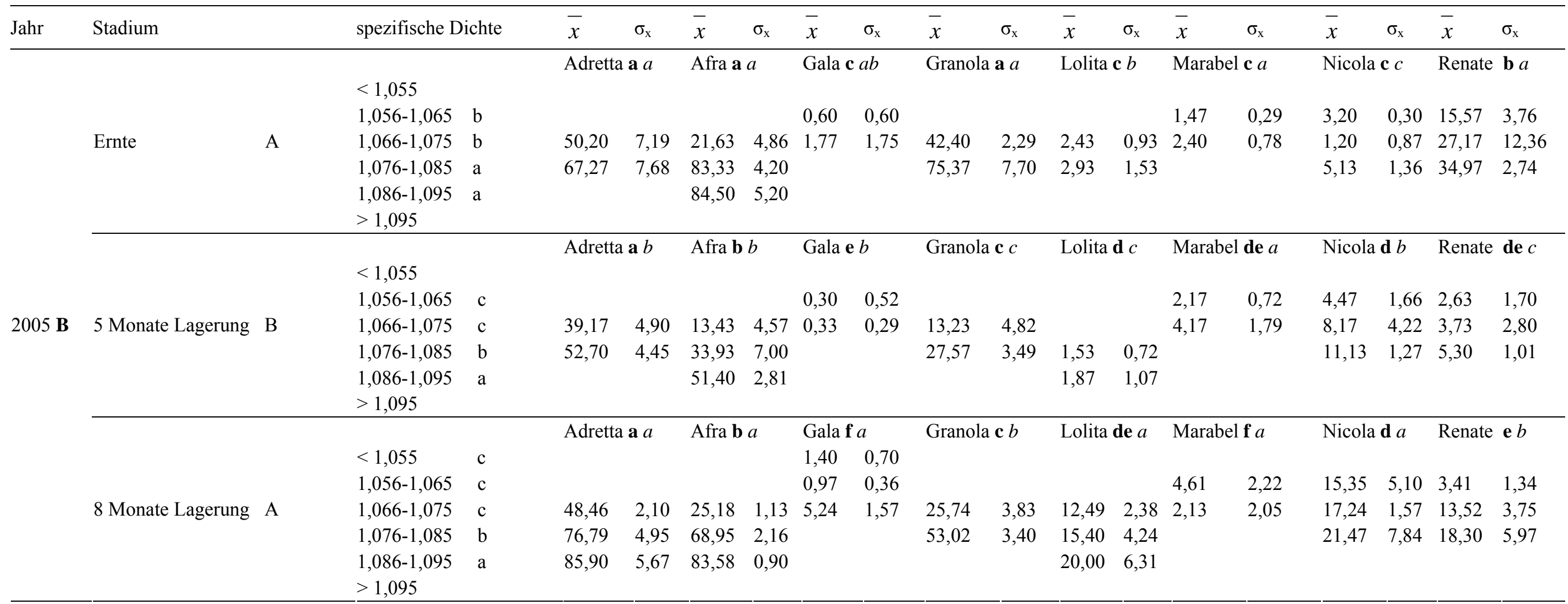


Tabelle 10.2 Schwarzfleckigkeit [Mittelwert $(\bar{x})$ und Standartabweichung $\left(\sigma_{\mathrm{x}}\right)$ ] $(\%)$ in Sorten und Knollen verschiedener spezifischer Dichten $\left(\mathrm{kg} \mathrm{L}^{-1}\right)$ nach der Vorernte und Ernte 2006 und nach fünf und acht Monaten Lagerung

\begin{tabular}{|c|c|c|c|c|c|c|c|c|c|c|c|c|c|c|c|c|c|c|c|c|}
\hline \multirow[t]{2}{*}{ Jahr } & \multicolumn{2}{|l|}{ Stadium } & \multicolumn{2}{|c|}{ spezifische Dichte } & $\bar{x}$ & $\sigma_{\mathrm{x}}$ & $\bar{x}$ & $\sigma_{\mathrm{x}}$ & $\overline{\bar{x}}$ & $\sigma_{\mathrm{x}}$ & $\overline{\bar{x}}$ & $\sigma_{\mathrm{x}}$ & $\overline{\bar{x}}$ & $\sigma_{\mathrm{x}}$ & $\overline{\bar{x}}$ & $\sigma_{\mathrm{x}}$ & $\overline{\bar{x}}$ & $\sigma_{\mathrm{x}}$ & $\bar{x}$ & $\sigma_{\mathrm{x}}$ \\
\hline & \multirow{6}{*}{ Vorernte } & & & \multirow[b]{2}{*}{ d } & \multicolumn{2}{|c|}{ Adretta a $d$} & \multicolumn{2}{|c|}{ Afra $\mathbf{b} b$} & \multicolumn{2}{|c|}{ Gala e $c$} & \multicolumn{2}{|c|}{ Granola $\mathbf{c} b$} & \multicolumn{2}{|c|}{ Lolita e $C$} & \multicolumn{2}{|c|}{ Marabel e $c$} & \multicolumn{2}{|c|}{ Nicola de $a b$} & \multicolumn{2}{|c|}{ Renate cd $c$} \\
\hline & & \multirow{5}{*}{$\mathrm{C}$} & \multirow{5}{*}{$\begin{array}{l}<1,055 \\
1,056-1,065 \\
1,066-1,075 \\
1,076-1,085 \\
1,086-1,095 \\
>1,095\end{array}$} & & & & & & 1,65 & 1,28 & & & 2,58 & 1,08 & 2,56 & 2,61 & 5,32 & 1,39 & & \\
\hline & & & & $\mathrm{c}$ & & & 15,71 & 0,63 & 2,39 & 0,57 & 9,61 & 0,20 & 0,90 & 1,04 & 2,55 & 1,22 & 5,05 & 2,82 & 8,68 & 1,79 \\
\hline & & & & $\mathrm{b}$ & 39,45 & 8,04 & 24,77 & 1,96 & & & & & 2,71 & 1,50 & 3,39 & 0,45 & & & 8,51 & 0,97 \\
\hline & & & & $\mathrm{a}$ & & & 23,18 & 1,12 & & & & & & & & & & & & \\
\hline & & & & $\mathrm{a}$ & & & & & & & & & & & & & & & & \\
\hline & & & $<1055$ & & Adrett & a $C$ & Afra $\mathbf{b}$ & & Gala c & & Grano & bc $b$ & Lolita & & Marab & & Nicola & $a$ & Renate & $c b$ \\
\hline & & & $1,056-1,065$ & $\mathrm{c}$ & & & & & 14,29 & 2,19 & 18,74 & 4,12 & 4,20 & 1,67 & 7,34 & 2,24 & 7,40 & 3,73 & 14,23 & 2,62 \\
\hline \multirow[t]{14}{*}{$2006 \mathbf{B}$} & Ernte & $\mathrm{B}$ & $1,066-1,075$ & $\mathrm{c}$ & & & 24,43 & 2,01 & 16,77 & 2,45 & 40,24 & 9,00 & 8,14 & 4,83 & 8,93 & 2,73 & 11,26 & 2,48 & 20,28 & 4,21 \\
\hline & & & $1,076-1,085$ & $\mathrm{~b}$ & 69,39 & 2,25 & 41,92 & 9,32 & & & & & & & & & 15,22 & 4,73 & 21,46 & 1,77 \\
\hline & & & $\begin{array}{l}1,086-1,095 \\
>1,095\end{array}$ & $\mathrm{a}$ & 97,86 & 1,36 & 81,50 & 4,86 & & & & & & & & & & & & \\
\hline & & & $<1,055$ & & Adrett & a $a$ & Afra $\mathbf{b}$ & & Gala d & & Grano & a $a$ & Lolita & & Marab & & Nicola & $\mathrm{d} a$ & Renat & c $a$ \\
\hline & & & $1,056-1,065$ & $\mathrm{~d}$ & & & & & 7,76 & 1,31 & 66,07 & 5,82 & 17,22 & 3,00 & 12,93 & 1,11 & 8,12 & 4,93 & 16,63 & 3,31 \\
\hline & 5 Monate Lagerung & A & $1,066-1,075$ & $\mathrm{c}$ & & & & & 10,37 & 1,47 & 93,74 & 3,14 & 27,25 & 3,85 & 12,41 & 1,04 & 14,04 & 3,92 & 29,05 & 6,01 \\
\hline & & & $1,076-1,085$ & $\mathrm{~b}$ & 93,21 & 3,92 & 32,99 & 4,72 & & & & & & & & & 21,87 & 2,91 & 32,73 & 4,70 \\
\hline & & & $\begin{array}{l}1,086-1,095 \\
>1,095\end{array}$ & $\mathrm{a}$ & 99,71 & 0,50 & 54,28 & 9,96 & & & & & & & & & & & & \\
\hline & & & $<1,055$ & & Adrett & a $a$ & Afra $\mathbf{b}$ & $d c$ & Gala c & d $c$ & Grano & b $b$ & Lolita & & Marab & $\mathbf{d} b c$ & Nicola & $b$ & Renat & $d c$ \\
\hline & & & $1,056-1,065$ & $\mathrm{e}$ & & & & & 2,17 & 1,75 & 8,37 & 2,40 & 3,33 & 3,06 & 3,83 & 1,02 & 2,37 & 1,17 & 4,43 & 2,22 \\
\hline & 8 Monate Lagerung & $\mathrm{BC}$ & $1,066-1,075$ & $\mathrm{~d}$ & & & & & 5,20 & 2,52 & 38,87 & 1,99 & 19,00 & 2,35 & 6,97 & 2,03 & 3,33 & 1,19 & 2,20 & 1,31 \\
\hline & & & $1,076-1,085$ & $\mathrm{c}$ & 86,37 & 1,18 & 11,83 & 2,66 & & & 25,33 & 1,72 & 30,23 & 6,35 & 11,33 & 4,88 & 5,93 & 0,49 & 10,17 & 2,15 \\
\hline & & & $1,086-1,095$ & $\mathrm{~b}$ & 95,20 & 2,17 & 21,00 & 5,03 & & & & & & & & & & & & \\
\hline & & & $>1,095$ & $\mathrm{a}$ & 97,40 & 0,82 & 55,00 & 3,39 & & & & & & & & & & & & \\
\hline
\end{tabular}


Tabelle 10.3 Schwarzfleckigkeit [Mittelwert $(\bar{x})$ und Standartabweichung $\left(\sigma_{\mathrm{x}}\right)$ ] $(\%)$ in Sorten und Knollen verschiedener spezifischer Dichten $\left(\mathrm{kg} \mathrm{L}^{-1}\right)$ nach der Vorernte und Ernte 2007 und nach fünf und acht Monaten Lagerung

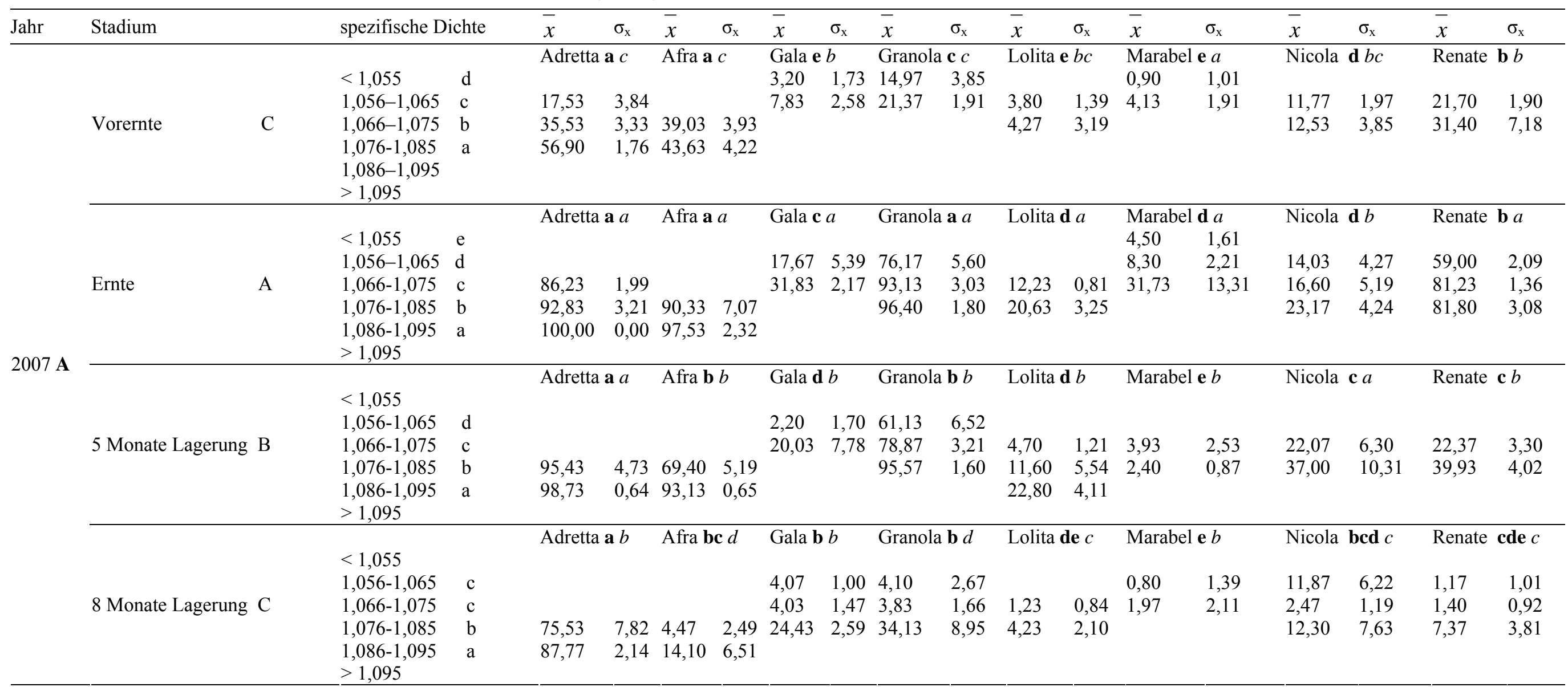


Tabelle 10.4 Schwarzfleckigkeit [Mittelwert $(\bar{x})$ und Standartabweichung $\left(\sigma_{\mathrm{x}}\right)$ ] $(\%)$ nach teilweiser Vorernte und Ernte in 2005,2006 und 2007 und nach fünf und acht Monaten Lagerung in Sorten, deren Knollen nicht nach spezifischen Dichten getrennt wurden aber teilweise bei $4^{\circ} \mathrm{C}$ und $8^{\circ} \mathrm{C}$ gelagert worden sind

\begin{tabular}{|c|c|c|c|c|c|c|c|c|c|c|c|c|c|c|c|c|c|c|c|c|}
\hline \multirow[b]{2}{*}{ Jahr } & \multirow[b]{2}{*}{ Stadium } & & \multirow{2}{*}{\multicolumn{2}{|c|}{${ }^{\circ} \mathrm{C}$}} & \multicolumn{2}{|c|}{ Adretta } & \multicolumn{2}{|c|}{ Afra } & \multicolumn{2}{|c|}{ Gala } & \multicolumn{2}{|c|}{ Granola } & \multicolumn{2}{|l|}{ Lolita } & \multicolumn{2}{|c|}{ Marabel } & \multicolumn{2}{|l|}{ Nicola } & \multicolumn{2}{|c|}{ Renate } \\
\hline & & & & & $\bar{x}$ & $\sigma_{\mathrm{x}}$ & $\bar{x}$ & $\sigma_{\mathrm{x}}$ & $\bar{x}$ & $\sigma_{\mathrm{x}}$ & $\bar{x}$ & $\sigma_{\mathrm{x}}$ & $\bar{x}$ & $\sigma_{\mathrm{x}}$ & $\bar{x}$ & $\sigma_{\mathrm{x}}$ & $\bar{x}$ & $\sigma_{\mathrm{x}}$ & $\bar{x}$ & $\sigma_{\mathrm{x}}$ \\
\hline \multirow{3}{*}{2005 AB } & Ernte & A & 4 & & $58,06 \mathbf{b} b$ & 3,95 & 82,93 a $a$ & 7,05 & $1,53 \mathbf{h} a$ & 0,83 & 49,86 с $b$ & 10,12 & 4,16 e $b$ & 2,15 & $2,33 \mathbf{g} a$ & 0,91 & 2,83 f $c$ & 1,41 & $38,76 \mathbf{d} a$ & 11,59 \\
\hline & 5 Monate Lagerung & $\mathrm{B}$ & 4 & & 41,53 a $c$ & 2,36 & $39,30 \mathbf{b} c$ & 2,53 & 0,93 h $a$ & 0,86 & 23,77 с $c$ & 1,86 & $2,17 \mathbf{g} b$ & 2,37 & 2,20 f $a$ & 1,18 & 7,73 d $b$ & 3,29 & 3,63 e $b$ & 0,92 \\
\hline & 8 Monate Lagerung & A & 4 & & 83,03 a $a$ & 4,08 & $68,80 \mathbf{b} b$ & 6,13 & 2,37 h $a$ & 0,74 & 58,21 с $a$ & 9,13 & 14,59 e $a$ & 0,91 & 3,52 g $a$ & 0,97 & $23,28 \mathbf{d} a$ & 5,14 & 5,82 f $b$ & 1,34 \\
\hline \multirow{4}{*}{$2006 \mathbf{B}$} & Vorernte & $\mathrm{C}$ & 4 & & 36,59 a $c$ & 6,51 & $16,46 \mathbf{b} d$ & 3,32 & 3,23 e $b$ & 0,80 & 11,09 c $c$ & 1,43 & 0,27 g $c$ & 0,47 & $0,80 \mathbf{f} c$ & 0,69 & 5,94 d $b$ & 1,64 & 11,59 с $c$ & 0,50 \\
\hline & Ernte & $\mathrm{B}$ & 4 & & 73,86 a $b$ & 6,51 & $65,63 \mathbf{b} a$ & 1,80 & $3,06 \mathbf{h} b$ & 0,75 & 28,3 с $b$ & 0,76 & $5,30 \mathbf{f} b$ & 1,02 & 10,03 e $a$ & 2,75 & $4,90 \quad \mathbf{g} \quad b$ & 2,02 & $19,93 \mathbf{d} b$ & 5,24 \\
\hline & 5 Monate Lagerung & $\mathrm{A}$ & 4 & & 92,74 a $a$ & 1,94 & 40,13 c $b$ & 3,30 & 9,21 f $a b$ & 4,12 & 58,44 b $a$ & 44,62 & 16,16 e $a$ & 1,90 & 10,79 f $a$ & 0,32 & 13,80 e $a$ & 6,23 & $33,40 \mathbf{d} a$ & 6,82 \\
\hline & 8 Monate Lagerung & $\mathrm{AB}$ & 4 & & 94,80 a $a$ & 2,12 & $25,93 \mathbf{b} c$ & 9,67 & $13,50 \mathbf{d} a$ & 6,66 & $26,26 \mathbf{b} b$ & 5,80 & 19,66 с $a$ & 2,40 & 5,23 e $b$ & 1,57 & 3,83 f $b$ & 2,15 & $15,96 \mathbf{d} b c$ & 10,76 \\
\hline \multirow{6}{*}{$2007 \mathbf{A}$} & Vorernte & $\mathrm{B}$ & 4 & & 48,13 a $c$ & 8,34 & 49,63 a $b$ & 20,21 & $5,60 \mathbf{d} c$ & 1,65 & $16,70 \mathbf{b} c$ & 2,36 & 3,00 de $c$ & 0,26 & 2,60 e $a$ & 1,15 & $12,13 \mathrm{c} b$ & 4,48 & $17,20 \mathbf{b} c$ & 4,52 \\
\hline & Ernte & A & 4 & & 93,33 a $a$ & 2,41 & 95,43 a $a$ & 2,07 & $26,83 \mathbf{d} a$ & 10,15 & $67,36 \mathrm{c} b$ & 5,28 & 18,93 e $a$ & 2,03 & $7,86 \mathbf{f} a$ & 1,41 & 19,36 e $a b$ & 4,28 & 75,10 b a & 1,73 \\
\hline & \multirow{2}{*}{5 Monate Lagerung } & \multirow{2}{*}{$\mathrm{A}$} & 4 & $\mathrm{a}$ & 93,50 a $a$ & 0,62 & $87,93 \mathbf{b} a$ & 4,60 & 12,83 e $b$ & 7,34 & $81,63 \mathbf{b} a$ & 8,02 & $9,13 \mathbf{f} b$ & 2,94 & 6,16 g $a$ & 4,90 & $24,33 \mathbf{d} a$ & 4,32 & 34,8 с $b$ & 6,48 \\
\hline & & & 8 & $\mathrm{a}$ & $90,16 \mathbf{a}$ & 5,24 & & & & & $93,36 \mathbf{a}$ & 2,78 & & & \multirow{3}{*}{$2,03 \mathbf{d} a$} & \multirow{3}{*}{0,81} & 19,13 c & 6,53 & $37,46 \mathbf{b}$ & 2,80 \\
\hline & \multirow{2}{*}{8 Monate Lagerung } & $P$ & 4 & $\mathrm{a}$ & 85,33 a $b$ & 3,30 & 8,00 с $c$ & 3,26 & $3,80 \mathbf{d} c$ & 2,30 & $19,53 \mathbf{b} c$ & 7,47 & $5,66 \mathrm{c} b c$ & 1,53 & & & $14,50 \mathbf{b} b$ & 1,37 & 5,60 c $d$ & 1,14 \\
\hline & & & 8 & $\mathrm{a}$ & $83,43 \mathbf{a}$ & 5,75 & $60,83 \mathbf{b}$ & 6,51 & $6,56 \mathrm{c}$ & 2,74 & & & $7,16 \mathrm{c}$ & 1,74 & & & & & & \\
\hline
\end{tabular}


Tabelle 10.5 Oxidatives Potential [Mittelwert $(\bar{x})$ und Standartabweichung $\left(\sigma_{\mathrm{x}}\right)$ ] $\left(\mathrm{AU}_{475 \mathrm{~nm}}\right)$ von Sorten und Knollen verschiedener spezifischer Dichten $\left(\mathrm{kg} \mathrm{L}^{-1}\right)$ nach der Ernte 2005 und nach fünf und acht Monaten Lagerung

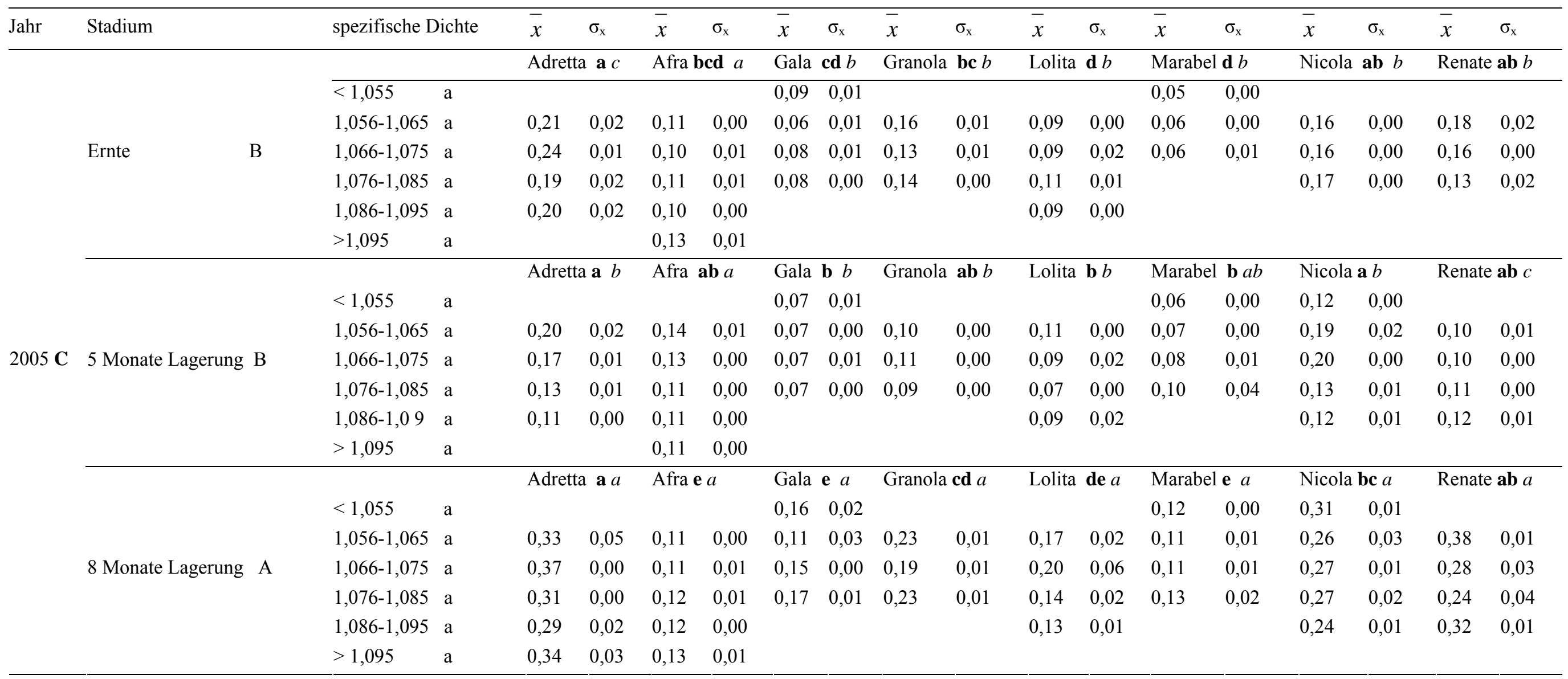


Tabelle 10.6 Oxidatives Potential [Mittelwert $(\bar{x})$ und Standartabweichung $\left(\sigma_{\mathrm{x}}\right)$ ] $\left(\mathrm{AU}_{475 \mathrm{~nm}}\right)$ von Sorten und Knollen verschiedener spezifischer Dichten $\left(\mathrm{kg} \mathrm{L}^{-1}\right)$ nach der Ernte 2006 und nach fünf und acht Monaten Lagerung

\begin{tabular}{|c|c|c|c|c|c|c|c|c|c|c|c|c|c|c|c|c|c|c|c|}
\hline \multirow[t]{2}{*}{ Jahr } & Stadium & \multicolumn{2}{|c|}{ spezifische Dichte } & $\bar{x}$ & $\sigma_{\mathrm{x}}$ & $\bar{x}$ & $\sigma_{\mathrm{x}}$ & $\bar{x}$ & $\sigma_{\mathrm{x}}$ & $\bar{x}$ & $\sigma_{\mathrm{x}}$ & $\bar{x}$ & $\sigma_{\mathrm{x}}$ & $\bar{x}$ & $\sigma_{\mathrm{x}}$ & $\bar{x}$ & $\sigma_{\mathrm{x}}$ & $\bar{x}$ & $\sigma_{\mathrm{x}}$ \\
\hline & & & & \multicolumn{2}{|c|}{ Adretta a $a$} & Afra & b $b$ & \multicolumn{2}{|c|}{ Gala $\mathbf{b} b$} & Granola b & b $b$ & Lolita 1 & \multirow[t]{2}{*}{ b $b$} & \multicolumn{2}{|c|}{ Marabel b $b$} & \multicolumn{2}{|c|}{ Nicola $\mathbf{b} b$} & \multicolumn{2}{|c|}{ Renate $\mathbf{b} b$} \\
\hline & & $<1,055$ & $\mathrm{a}$ & & & & & 0,13 & 0,00 & & & & & 0,08 & 0,01 & & & & \\
\hline & & $1,056-1,065$ & $\mathrm{a}$ & 0,44 & 0,23 & & & 0,10 & 0,01 & 0,18 & 0,01 & 0,09 & 0,01 & 0,07 & 0,00 & 0,13 & 0,00 & 0,15 & 0,01 \\
\hline & \multirow[t]{4}{*}{ Ernte } & $1,066-1,075$ & $\mathrm{a}$ & 0,32 & 0,01 & 0,13 & 0,01 & 0,10 & 0,00 & 0,16 & 0,00 & 0,08 & 0,00 & 0,12 & 0,05 & 0,15 & 0,00 & 0,14 & 0,02 \\
\hline & & $1,076-1,085$ & $\mathrm{a}$ & 0,29 & 0,01 & 0,11 & 0,00 & 0,10 & 0,00 & 0,15 & 0,01 & 0,10 & 0,00 & 0,07 & 0,00 & 0,14 & 0,00 & 0,12 & 0,01 \\
\hline & & $1,086-1,095$ & $\mathrm{a}$ & 0,28 & 0,03 & 0,11 & 0,00 & & & & & & & & & & & 0,10 & 0,01 \\
\hline & & $>1,095$ & $\mathrm{a}$ & 0,24 & 0,01 & 0,10 & 0,01 & & & & & & & & & & & & \\
\hline & & & & Adret & a $a$ & Afra & b $b$ & Gala & $\mathrm{cd} b$ & Granola $\mathbf{b}$ & b $b$ & Lolita & b $b$ & Mara & $1 \mathrm{~d} b$ & Nicol & bc $b$ & Renate & b $b$ \\
\hline & & $<1,055$ & $\mathrm{a}$ & & & & & 0,11 & 0,01 & & & & & & & & & & \\
\hline & & $1,056-1,065$ & $\mathrm{a}$ & 0,31 & 0,00 & & & 0,09 & 0,00 & 0,15 & 0,01 & 0,13 & 0,00 & 0,09 & 0,00 & 0,13 & 0,00 & 0,19 & 0,01 \\
\hline \multirow[t]{11}{*}{2006 B } & \multirow[t]{4}{*}{5 Monate Lagerung B } & $1,066-1,075$ & $\mathrm{a}$ & 0,25 & 0,01 & 0,12 & 0,01 & 0,09 & 0,00 & 0,14 & 0,03 & 0,15 & 0,01 & 0,07 & 0,00 & 0,15 & 0,01 & 0,16 & 0,00 \\
\hline & & $1,076-1,085$ & $\mathrm{a}$ & 0,28 & 0,01 & 0,12 & 0,00 & 0,07 & 0,00 & 0,18 & 0,01 & 0,17 & 0,01 & 0,09 & 0,01 & 0,13 & 0,00 & 0,16 & 0,01 \\
\hline & & $1,086-1,095$ & $\mathrm{a}$ & 0,22 & 0,00 & 0,13 & 0,00 & & & & & 0,19 & 0,01 & & & 0,16 & 0,01 & 0,17 & 0,01 \\
\hline & & $>1,095$ & $\mathrm{a}$ & 0,27 & 0,01 & 0,10 & 0,01 & & & & & & & & & & & & \\
\hline & \multirow{7}{*}{8 Monate Lagerung A } & & & \multicolumn{2}{|c|}{ Adretta a $a$} & \multicolumn{2}{|c|}{ Afra $\mathbf{b} a$} & \multicolumn{2}{|c|}{ Gala b $a$} & \multicolumn{2}{|c|}{ Granola ab $a$} & \multicolumn{2}{|c|}{ Lolita $\mathbf{b} a$} & \multicolumn{2}{|c|}{ Marabel b $a$} & \multicolumn{2}{|c|}{ Nicola b $a b$} & \multirow{2}{*}{\multicolumn{2}{|c|}{ Renate b $a$}} \\
\hline & & $<1,055$ & $\mathrm{a}$ & & & & & 0,23 & 0,00 & & & & & & & & & & \\
\hline & & $1,056-1,065$ & $\mathrm{a}$ & & & & & 0,18 & 0,01 & 0,28 & 0,01 & 0,20 & 0,01 & 0,18 & 0,01 & 0,19 & 0,00 & 0,34 & 0,02 \\
\hline & & $1,066-1,075$ & $\mathrm{a}$ & 0,37 & 0,04 & 0,18 & 0,00 & 0,16 & 0,00 & 0,30 & 0,01 & 0,21 & 0,01 & 0,16 & 0,01 & 0,21 & 0,01 & 0,30 & 0,01 \\
\hline & & $1,076-1,085$ & $\mathrm{a}$ & 0,40 & 0,02 & 0,14 & 0,02 & & & 0,25 & 0,00 & 0,20 & 0,01 & 0,16 & 0,00 & 0,24 & 0,01 & 0,16 & 0,02 \\
\hline & & $1,086-1,095$ & $\mathrm{a}$ & 0,35 & 0,01 & 0,16 & 0,00 & & & & & & & 0,16 & 0,01 & & & 0,26 & 0,03 \\
\hline & & $>1,095$ & $\mathrm{a}$ & 0,31 & 0,01 & 0,16 & 0,01 & & & & & & & & & & & & \\
\hline
\end{tabular}


Tabelle 10.7 Antioxidative Kapazität (FRAP) [Mittelwert $(\bar{x})$ und Standartabweichung $\left(\sigma_{\mathrm{x}}\right)$ ] $\left(\mathrm{mmol} \mathrm{kg}^{-1}\right.$ FM) von Sorten und Knollen verschiedener spezifischer Dichten $\left(\mathrm{kg} \mathrm{L}^{-1}\right)$ nach der Ernte 2005 und nach fünf und acht Monaten Lagerung

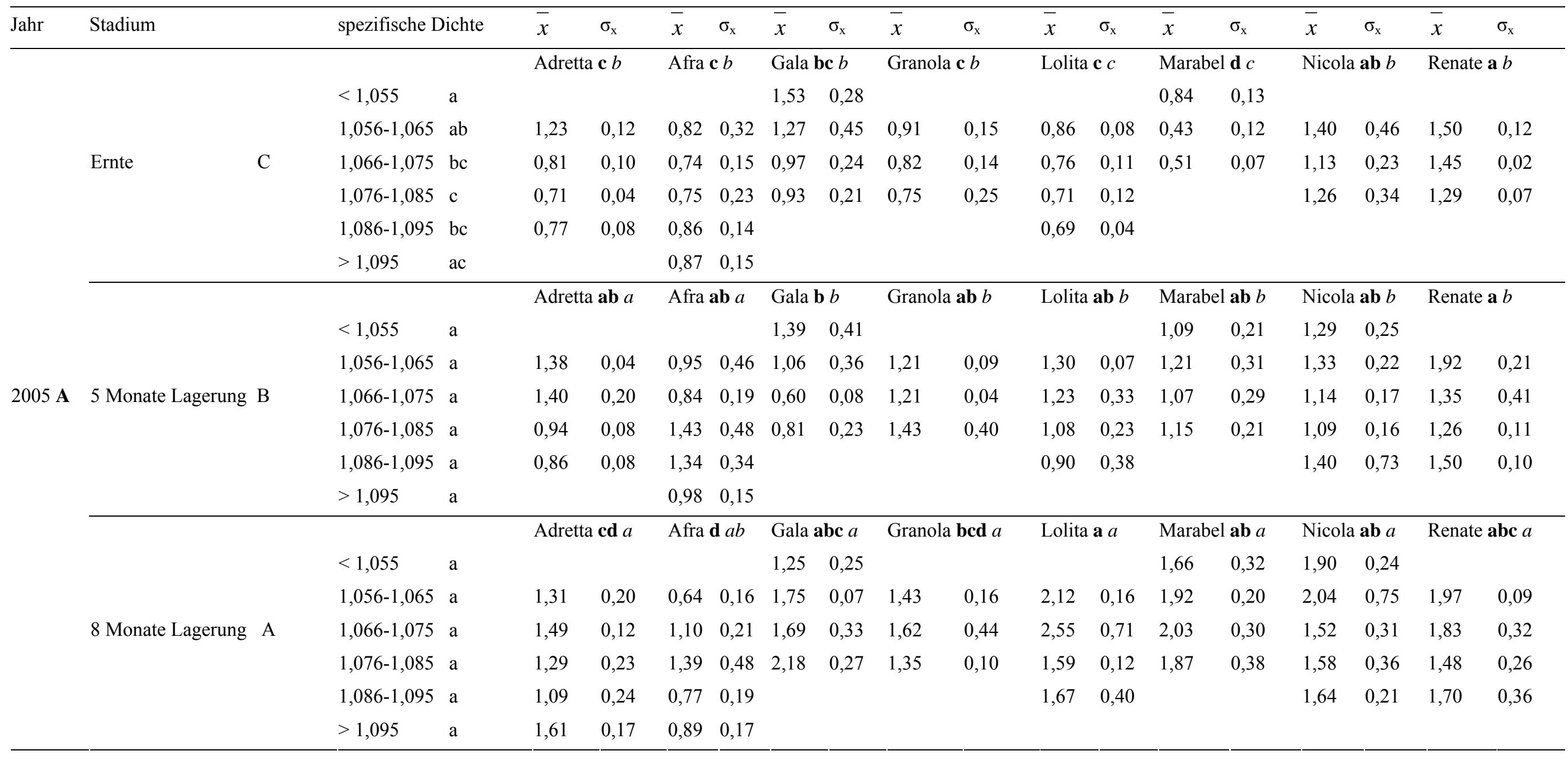


Tabelle 10.8 Antioxidative Kapazität (FRAP) [Mittelwert $(\bar{x})$ und Standartabweichung $\left(\sigma_{\mathrm{x}}\right)$ ] ( $\left.\mathrm{mmol} \mathrm{kg}^{-1} \mathrm{FM}\right)$ von Sorten und Knollen verschiedener spezifischer Dichten $\left(\mathrm{kg} \mathrm{L}^{-1}\right)$ nach der Ernte 2006 und nach fünf und acht Monaten Lagerung

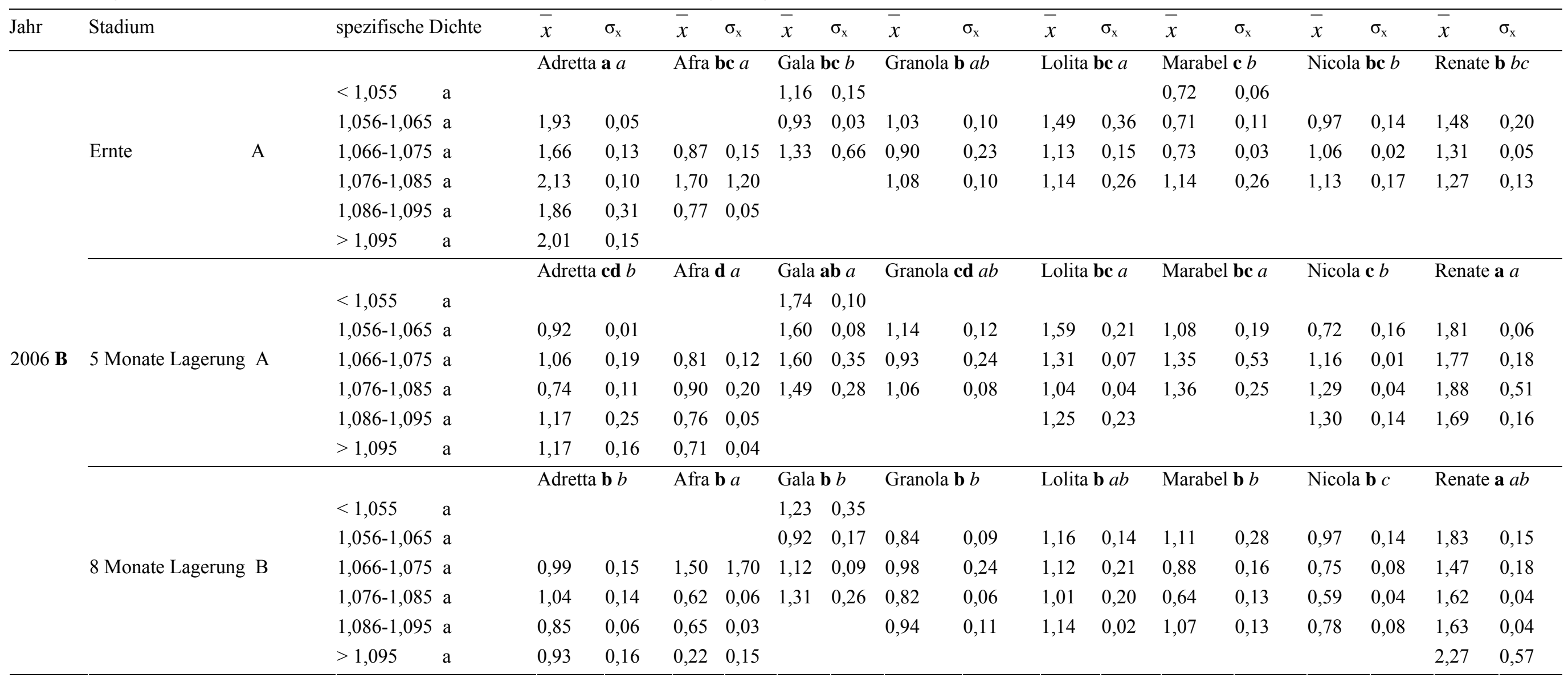


Tabelle 10.9 Antioxidative Kapazität (FRAP) [Mittelwert $(\bar{x})$ und Standartabweichung $\left(\sigma_{\mathrm{x}}\right)$ ] $(\%)$ von Schalen- und Markgewebe nach der Ernte der Sorten in 2005 und 2006, die Knollen wurden nicht nach spezifischen Dichten getrennt

\begin{tabular}{|c|c|c|c|c|c|c|c|c|c|c|c|c|c|c|c|c|c|c|}
\hline Jahr & Stadium & Gewebe & $\bar{x}$ & $\sigma_{\mathrm{x}}$ & $\bar{x}$ & $\sigma_{\mathrm{x}}$ & $\bar{x}$ & $\sigma_{\mathrm{x}}$ & $\bar{x}$ & $\sigma_{\mathrm{x}}$ & $\bar{x}$ & $\sigma_{\mathrm{x}}$ & $\bar{x}$ & $\sigma_{\mathrm{x}}$ & $\bar{x}$ & $\sigma_{\mathrm{x}}$ & $\bar{x}$ & $\sigma_{\mathrm{x}}$ \\
\hline & & & \multicolumn{2}{|c|}{ Adretta $\mathbf{a b} a$} & \multicolumn{2}{|c|}{ Afra $\mathbf{a b} a$} & \multicolumn{2}{|c|}{ Gala a $a$} & \multicolumn{2}{|c|}{ Granola $\mathbf{b} b$} & \multicolumn{2}{|c|}{ Lolita $\mathbf{a b} b$} & \multicolumn{2}{|c|}{ Marabel $\mathbf{b} b$} & \multicolumn{2}{|c|}{ Nicola ab $b$} & \multicolumn{2}{|c|}{ Renate a $b$} \\
\hline \multirow[t]{3}{*}{$2005 \mathrm{~A}$} & Ernte & Mark b & 1,00 & 0,05 & 0,89 & 0,07 & 1,18 & 0,12 & 0,64 & 0,18 & 0,72 & 0,03 & 0,74 & 0,00 & 0,86 & 0,16 & 1,13 & 0,34 \\
\hline & & Schale a & 1,24 & 0,19 & 1,39 & 0,28 & 1,63 & 0,47 & 1,24 & 0,16 & 1,52 & 0,21 & 1,13 & 0,12 & 1,44 & 0,35 & 1,73 & 0,15 \\
\hline & & & \multicolumn{2}{|c|}{ Adretta $\mathbf{a b} b$} & \multicolumn{2}{|c|}{ Afra $\mathbf{a b} a$} & \multicolumn{2}{|c|}{ Gala b $b$} & \multicolumn{2}{|c|}{ Granola ab $a$} & \multicolumn{2}{|c|}{ Lolita a $a$} & \multicolumn{2}{|c|}{ Marabel $\mathbf{b} b$} & \multicolumn{2}{|c|}{ Nicola ab $a b$} & \multicolumn{2}{|c|}{ Renate a $a$} \\
\hline \multirow[t]{2}{*}{2006 B } & Ernte & Mark b & 0,98 & 0,12 & 1,27 & 0,79 & 0,99 & 0,33 & 1,41 & 0,09 & 1,35 & 0,19 & 0,60 & 0,03 & 1,03 & 0,15 & 1,08 & 0,11 \\
\hline & & Schale a & 1,83 & 0,26 & 1,26 & 0,06 & 1,06 & 0,12 & 1,35 & 0,36 & 1,84 & 0,09 & 1,38 & 0,10 & 1,31 & 0,03 & 2,02 & 0,06 \\
\hline
\end{tabular}

Tabelle 10.10 Antioxidative Kapazität (H-ORAC) [Mittelwert $(\bar{x})$ und Standartabweichung $\left(\sigma_{\mathrm{x}}\right)$ ] (mmol GAE kg ${ }^{-1}$ FM) von Schalen- und Markgewebe nach der Ernte der Sorten in 2005 und 2006, die Knollen wurden nicht nach spezifischen Dichten getrennt

\begin{tabular}{|c|c|c|c|c|c|c|c|c|c|c|c|c|c|c|c|c|c|c|}
\hline Jahr & Stadium & Gewebe & $\bar{x}$ & $\sigma_{\mathrm{x}}$ & $\bar{x}$ & $\sigma_{\mathrm{x}}$ & $\bar{x}$ & $\sigma_{\mathrm{x}}$ & $\bar{x}$ & $\sigma_{\mathrm{x}}$ & $\bar{x}$ & $\sigma_{\mathrm{x}}$ & $\bar{x}$ & $\sigma_{\mathrm{x}}$ & $\bar{x}$ & $\sigma_{\mathrm{x}}$ & $\bar{x}$ & $\sigma_{\mathrm{x}}$ \\
\hline & & & Adretta & & Afra $c$ & & Gala & $\mathrm{d} a$ & Grano & ab $a$ & Lolita & & Marabe & & Nicola & $a$ & Renat & $a$ \\
\hline \multirow[t]{3}{*}{2005 B } & Ernte & Mark b & 42,09 & 3,53 & 29,77 & 2,14 & 33,55 & 4,61 & 31,85 & 2,00 & 32,80 & 2,11 & 24,63 & 3,38 & 23,11 & 5,92 & 42,88 & 9,53 \\
\hline & & Schale a & 59,59 & 4,86 & 38,15 & 3,25 & 37,53 & 3,20 & 57,94 & 5,80 & 48,21 & 2,29 & 47,41 & 0,83 & 30,99 & 3,37 & 56,46 & 7,31 \\
\hline & & & Adretta & & Afra $\mathbf{b}$ & & Gala & & Grano & DC & Lolita & & Marabe & C & Nicola & $\mathrm{C}$ & Renat & \\
\hline \multirow[t]{2}{*}{2006 A } & Ernte & Mark b & 55,02 & 9,07 & 47,01 & 7,16 & 45,16 & 3,05 & 33,26 & 4,38 & 55,01 & 3,97 & 27,04 & 2,60 & 44,32 & 4,92 & 30,47 & 2,53 \\
\hline & & Schale a & 109,47 & 6,34 & 64,34 & 8,57 & 53,75 & 2,39 & 75,74 & 1,87 & 72,41 & 2,63 & 76,33 & 4,33 & 65,81 & 3,95 & 41,93 & 3,39 \\
\hline
\end{tabular}


Tabelle 10.11 Wasserkonzentration [Mittelwert $(\bar{x})$ und Standartabweichung $\left(\sigma_{\mathrm{x}}\right)$ ] $\left(\mathrm{g} \mathrm{kg}^{-1} \mathrm{FM}\right)$ in Sorten und Knollen verschiedener spezifischer Dichten $\left(\mathrm{kg} \mathrm{L}^{-1}\right)$ nach der Ernte 2006 und nach fünf und acht Monaten Lagerung

\begin{tabular}{|c|c|c|c|c|c|c|c|c|c|c|c|c|c|c|c|c|c|c|c|}
\hline Jahr & Stadium & & spezifische Dichte & $\bar{x}$ & $\sigma_{\mathrm{x}}$ & $\bar{x}$ & $\sigma_{\mathrm{x}}$ & $\bar{x}$ & $\sigma_{\mathrm{x}}$ & $\bar{x}$ & $\sigma_{\mathrm{x}}$ & $\bar{x}$ & $\sigma_{\mathrm{x}}$ & $\bar{x}$ & $\sigma_{\mathrm{x}}$ & $\bar{x}$ & $\sigma_{\mathrm{x}}$ & $\bar{x}$ & $\sigma_{\mathrm{x}}$ \\
\hline & & & & Adretta & a $a$ & Afra & a $a$ & Gala & a $a$ & Granola & a $a$ & Lolita & a $a$ & Marabel & a $a$ & Nicola & a $a$ & Renate & a $a$ \\
\hline & & & $<1,055$ & & & & & & & & & & & & & & & & \\
\hline & & & $1,056-1,06$ a & & & & & 862,91 & 50,69 & 848,86 & 33,51 & 833,56 & 21,24 & 847,08 & 54,30 & 845,77 & 39,65 & 831,87 & 24,85 \\
\hline & Ernte & A & $1,066-1,075 \mathrm{ab}$ & & & 822,30 & 35,24 & 810,71 & 31,18 & 810,10 & 40,74 & 801,36 & 15,05 & 802,87 & 12,66 & 840,02 & 94,68 & 825,54 & 28,92 \\
\hline & & & $1,076-1,085 \mathrm{~b}$ & 802,87 & 48,43 & 812,63 & 66,24 & & & & & & & & & 787,88 & 8,07 & 806,34 & 104,71 \\
\hline & & & $1,086-1,095 \mathrm{~b}$ & 786,69 & 33,11 & 784,95 & 32,15 & & & & & & & & & & & & \\
\hline & & & $>1,095$ & & & & & & & & & & & & & & & & \\
\hline & & & & Adretta & c $a$ & Afra & c $a$ & Gala & a $a$ & Granola & ab $a$ & Lolita & bc $a$ & Marabel & bc $a$ & Nicola & $\mathbf{a b} a$ & Renate & bc $a$ \\
\hline & & & $<1,055 \quad a b$ & & & & & 892,12 & 26,45 & & & & & & & & & & \\
\hline & & & $1,056-1,065 \mathrm{~b}$ & 850,59 & 26,71 & & & 856,99 & 7,39 & 863,30 & 41,69 & 847,81 & 23,20 & 819,18 & 21,61 & 871,11 & 20,19 & 818,41 & 14,65 \\
\hline \multirow[t]{11}{*}{2006 A } & 5 Monate Lagerung & A & $1,066-1,075 \mathrm{bc}$ & 816,29 & 45,43 & 823,44 & 24,73 & 830,10 & 26,58 & 818,42 & 36,16 & 819,29 & 32,83 & 816,43 & 23,89 & 885,26 & 62,39 & 823,03 & 22,91 \\
\hline & & & $1,076-1,085 \mathrm{~cd}$ & 773,94 & 54,37 & 812,51 & 34,31 & 843,58 & 43,27 & 822,15 & 19,32 & 812,73 & 46,31 & 798,01 & 8,65 & 767,82 & 81,92 & 802,76 & 23,78 \\
\hline & & & $1,086-1,095 \mathrm{de}$ & 764,25 & 18,54 & 774,49 & 23,21 & & & & & 793,32 & 8,12 & & & 832,38 & 95,30 & 786,67 & 35,71 \\
\hline & & & $>1,095$ & 770,68 & 15,08 & 752,73 & 19,38 & & & & & & & & & & & & \\
\hline & & & & Adretta & b $a$ & Afra & b $a$ & Gala & a $a$ & Granola & ab $a$ & Lolita & $\mathbf{a b} a$ & Marabel & $\mathbf{a b} a$ & Nicola & a $a$ & Renate & $\mathbf{a b} a$ \\
\hline & & & $<1,055$ & & & & & 865,29 & 28,18 & & & & & & & 852,84 & 21,39 & & \\
\hline & & & $1,056-1,065 \mathrm{ab}$ & 819,95 & 43,14 & & & 833,13 & 22,07 & 819,76 & 35,03 & 856,23 & 40,75 & 827,67 & 31,11 & 828,69 & 26,07 & 845,72 & 22,76 \\
\hline & 8 Monate Lagerung & A & $1,066-1,075 \mathrm{bc}$ & 787,35 & 16,11 & 811,31 & 22,04 & 796,33 & 58,59 & 836,84 & 39,13 & 807,05 & 13,78 & 806,46 & 51,75 & 801,00 & 22,18 & 814,39 & 12,20 \\
\hline & & & $1,076-1,085 \mathrm{~cd}$ & 775,46 & 24,72 & 761,01 & 15,36 & & & 781,32 & 16,44 & 791,15 & 12,44 & 809,94 & 55,90 & & & 809,03 & 16,54 \\
\hline & & & $1,086-1,095 \mathrm{~d}$ & 736,82 & 35,17 & 781,61 & 22,29 & & & 779,00 & 23,11 & 753,59 & 61,29 & 755,10 & 61,15 & & & 763,41 & 43,13 \\
\hline & & & $>1,095$ & & & 748,90 & 21,25 & & & & & & & & & & & 783,91 & 26,95 \\
\hline
\end{tabular}


Tabelle 10.12 Wasserkonzentration [Mittelwert $(\bar{x})$ und Standartabweichung $\left(\sigma_{\mathrm{x}}\right)$ ] $\left(\mathrm{g} \mathrm{kg}^{-1} \mathrm{FM}\right)$ in Sorten und Knollen verschiedener spezifischer Dichten $\left(\mathrm{kg} \mathrm{L}^{-1}\right)$ nach der Ernte 2007 und nach fünf und acht Monaten Lagerung

\begin{tabular}{|c|c|c|c|c|c|c|c|c|c|c|c|c|c|c|c|c|c|c|}
\hline Jahr & Stadium & spezifische Dichte & $\bar{x}$ & $\sigma_{\mathrm{x}}$ & $\bar{x}$ & $\sigma_{\mathrm{x}}$ & $\bar{x}$ & $\sigma_{\mathrm{x}}$ & $\bar{x}$ & $\sigma_{\mathrm{x}}$ & $\bar{x}$ & $\sigma_{\mathrm{x}}$ & $\bar{x}$ & $\sigma_{\mathrm{x}}$ & $\bar{x}$ & $\sigma_{\mathrm{x}}$ & $\bar{x}$ & $\sigma_{\mathrm{x}}$ \\
\hline & & & Adretta & a $a$ & Afra & a $a$ & Gala & a $a$ & Granola & a $a$ & Lolita & a $a$ & Marabel & a $a$ & Nicola & a $a$ & Renate & a $a$ \\
\hline & & $<1,055$ & & & & & 861,58 & 37,17 & & & & & 891,95 & 19,25 & & & & \\
\hline & & $1,056-1,065 \mathrm{ab}$ & 839,34 & 23,01 & & & 842,58 & 17,45 & 839,27 & 52,20 & 805,03 & 45,87 & 865,42 & 27,54 & 833,84 & 18,90 & 843,90 & 40,21 \\
\hline & Ernte & $1,066-1,075 \mathrm{abc}$ & 811,86 & 47,62 & 817,97 & 17,80 & 844,28 & 39,64 & 848,52 & 263,84 & 826,31 & 39,11 & 822,96 & 24,08 & 823,52 & 39,50 & 823,45 & 12,80 \\
\hline & & $1,076-1,085$ bc & 813,65 & 43,82 & 796,37 & 113,70 & 801,46 & 35,02 & 809,21 & 18,23 & 804,76 & 32,11 & 813,12 & 8,28 & 797,75 & 16,86 & 789,87 & 21,86 \\
\hline & & $1,086-1,095 \mathrm{bc}$ & 770,59 & 20,11 & 778,94 & 31,66 & & & & & & & & & & & & \\
\hline & & $>1,095$ & 757,13 & 38,40 & & & & & & & & & & & & & & \\
\hline & & & Adretta & d $a$ & Afra & $\operatorname{cd} a$ & Gala & a $a$ & Granola & abc $a$ & Lolita & abcd $a$ & Marabel & ab $a$ & Nicola & abcd $a$ & Renate & bcd $a$ \\
\hline & & $<1,055$ & & & & & 863,10 & 14,80 & & & & & & & & & & \\
\hline & & $1,056-1,065 \mathrm{ab}$ & & & & & 856,14 & 28,83 & 843,51 & 30,49 & 847,05 & 39,78 & 847,51 & 11,21 & 838,61 & 23,69 & 846,73 & 28,40 \\
\hline \multirow[t]{11}{*}{2007 A } & 5 Monate Lagerung A & $1,066-1,075$ bc & 806,11 & 29,13 & 849,76 & 17,06 & 832,70 & 33,71 & 841,85 & 20,36 & 827,41 & 47,55 & 842,19 & 16,36 & 824,21 & 17,60 & 824,99 & 26,16 \\
\hline & & $1,076-1,085 \mathrm{c}$ & 822,78 & 30,71 & 819,90 & 18,23 & 816,86 & 35,55 & 821,13 & 22,46 & 809,81 & 13,59 & 826,91 & 8,83 & 813,74 & 23,63 & 805,48 & 22,36 \\
\hline & & $1,086-1,095 \mathrm{~d}$ & 779,73 & 31,22 & 790,51 & 15,92 & & & 812,31 & 8,09 & 777,87 & 11,03 & & & 778,54 & 20,89 & 773,58 & 18,22 \\
\hline & & $>1,095$ & 771,74 & 30,54 & 758,75 & 6,44 & & & & & & & & & & & & \\
\hline & & & Adretta & a $a$ & Afra & ab $a$ & Gala & a $a$ & Granola & ab $a$ & Lolita & b $a$ & Marabel & ab $a$ & Nicola & ab $a$ & Renate & ab $a$ \\
\hline & & $<1,055$ & & & & & 861,59 & 21,70 & 794,32 & 34,60 & & & & & & & & \\
\hline & & $1,056-1,065 \mathrm{ab}$ & 852,75 & 26,26 & & & 828,29 & 36,37 & 836,88 & 41,03 & 750,99 & 3,18 & 827,47 & 15,39 & 804,65 & 10,70 & 816,44 & 47,78 \\
\hline & 8 Monate Lagerung A & $1,066-1,075 \mathrm{ab}$ & 810,83 & 39,22 & 822,33 & 27,52 & 817,55 & 21,97 & 794,76 & 21,93 & 787,61 & 49,26 & 811,91 & 26,92 & 783,55 & 28,49 & 778,71 & 7,21 \\
\hline & & $1,076-1,085 \mathrm{ab}$ & 821,70 & 14,30 & 789,64 & 12,75 & 812,36 & 9,22 & 794,54 & 15,76 & 766,56 & 59,37 & 793,36 & 17,56 & 786,21 & 45,92 & 781,74 & 20,83 \\
\hline & & $1,086-1,095 \mathrm{ab}$ & 787,88 & 25,99 & 777,23 & 27,72 & & & & & & & & & 757,88 & 9,02 & & \\
\hline & & $>1,095$ & 743,06 & 27,32 & 750,60 & 16,06 & & & & & & & & & & & & \\
\hline
\end{tabular}


Tabelle 10.13 Wasserkonzentration [Mittelwert $(\bar{x})$ und Standartabweichung $\left(\sigma_{\mathrm{x}}\right)$ ] $\left(\mathrm{g} \mathrm{kg}^{-1} \mathrm{FM}\right)$ in Kronen- und Nabelgewebe nach der Ernte 2006 und 2007 und nach fünf und acht Monaten Lagerung der Sorten, deren Knollen nicht nach spezifischen Dichten getrennt wurden

\begin{tabular}{|c|c|c|c|c|c|c|c|c|c|c|c|c|c|c|c|c|c|c|c|}
\hline Jahr & Stadium & & Gewebe & $\bar{x}$ & $\sigma_{\mathrm{x}}$ & $\bar{x}$ & $\sigma_{\mathrm{x}}$ & $\bar{x}$ & $\sigma_{\mathrm{x}}$ & $\bar{x}$ & $\sigma_{\mathrm{x}}$ & $\bar{x}$ & $\sigma_{\mathrm{x}}$ & $\bar{x}$ & $\sigma_{\mathrm{x}}$ & $\bar{x}$ & $\sigma_{\mathrm{x}}$ & $\bar{x}$ & $\sigma_{\mathrm{x}}$ \\
\hline & & & & Adretta & a $a$ & Afra & a $a$ & Gala & a $a$ & Granola & a $a$ & Lolita & a $a$ & Marabel & a $a$ & Nicola & a $a$ & Renate & a $a$ \\
\hline & Ernte & A & Nabel a & 801,43 & 6,37 & 819,64 & 21,78 & 868,62 & 36,93 & 843,82 & 26,94 & 835,31 & 10,32 & 838,07 & 23,44 & 842,14 & 5,48 & 827,25 & 40,28 \\
\hline & & & Krone b & 781,97 & 7,30 & 785,85 & 8,40 & 831,98 & 1,76 & 828,98 & 43,81 & 810,15 & 1,19 & 811,93 & 8,61 & 801,91 & 17,30 & 802,64 & 29,27 \\
\hline & & & & Adretta & a $a$ & Afra & a $a$ & Gala & a $a$ & Granola & a $a$ & Lolita & a $a$ & Marabel & a $a$ & Nicola & a $a$ & Renate & a $a$ \\
\hline \multirow[t]{9}{*}{2006 A } & 5 Monate Lagerung & A & Nabel a & 804,47 & 3,79 & 803,98 & 36,86 & 863,71 & 24,20 & 855,41 & 0,25 & 858,28 & 19,74 & 832,92 & 30,02 & 825,68 & 33,25 & 815,73 & 6,10 \\
\hline & & & Krone b & 790,70 & 7,40 & 787,22 & 49,73 & 833,54 & 13,40 & 831,49 & 47,95 & 798,74 & 31,98 & 817,89 & 43,34 & 809,33 & 21,06 & 769,50 & 29,44 \\
\hline & & & & Adretta & a $a$ & Afra & a $a$ & Gala & a $a$ & Granola & a $a$ & Lolita & a $a$ & Marabel & a $a$ & Nicola & a $a$ & Renate & a $a$ \\
\hline & 8 Monate Lagerung & A & Nabel a & 801,49 & 1,29 & 783,44 & 10,90 & 859,25 & 29,59 & 891,99 & 40,23 & 755,62 & 96,61 & 792,11 & 11,85 & 795,57 & 3,42 & 847,62 & 9,79 \\
\hline & & & Krone a & 815,64 & 74,95 & 748,42 & 70,85 & 828,39 & 9,53 & 807,42 & 11,51 & 931,53 & 48,09 & 798,60 & 27,04 & 819,38 & 23,51 & 773,14 & 42,86 \\
\hline & & & & Adretta & a $a$ & Afra & a $a$ & Gala & a $a$ & Granola & a $a$ & Lolita & a $a$ & Marabel & a $a$ & Nicola & a $a$ & Renate & a $a$ \\
\hline & Ernte & A & Nabel a & 813,56 & 1,05 & 767,67 & 19,50 & 889,72 & 60,87 & 865,69 & 8,42 & 856,02 & 28,28 & 880,63 & 45,92 & 819,05 & 22,76 & 802,65 & 17,47 \\
\hline & & & Krone $\mathrm{b}$ & 801,16 & 9,84 & 800,86 & 4,81 & 773,17 & 43,64 & 801,61 & 8,80 & 776,54 & 15,42 & 848,41 & 14,53 & 797,55 & 1,66 & 805,94 & 21,52 \\
\hline & & & & Adretta & a $a$ & Afra & a $a$ & Gala & a $a$ & Granola & a $a$ & Lolita & a $a$ & Marabel & a $a$ & Nicola & a $a$ & Renate & a $a$ \\
\hline \multirow[t]{5}{*}{2007 A } & 5 Monate Lagerung & A & Nabel a & 835,86 & 13,48 & 813,91 & 3,95 & 815,66 & 6,92 & 841,78 & 39,58 & 810,07 & 11,60 & 824,91 & 21,43 & 843,55 & 10,49 & 814,10 & 19,12 \\
\hline & & & Krone a & 776,97 & 25,45 & 778,76 & 17,52 & 872,00 & 23,96 & 810,05 & 3,14 & 818,15 & 32,88 & 835,21 & 15,47 & 822,22 & 24,00 & 818,64 & 2,34 \\
\hline & & & & Adretta & a $a$ & Afra & a $a$ & Gala & a $a$ & Granola & a $a$ & Lolita & a $a$ & Marabel & a $a$ & Nicola & a $a$ & Renate & a $a$ \\
\hline & 8 Monate Lagerung & A & Nabel a & 785,96 & 55,16 & 819,08 & 35,47 & 809,25 & 17,32 & 825,08 & 2,68 & 808,08 & 16,65 & 843,63 & 34,09 & 790,42 & 44,29 & 832,80 & 15,69 \\
\hline & & & Krone a & 790,72 & 11,65 & 777,80 & 32,51 & 802,89 & 22,48 & 809,87 & 20,00 & 785,87 & 6,62 & 847,76 & 0,31 & 798,21 & 13,66 & 801,91 & 21,95 \\
\hline
\end{tabular}


Tabelle 10.14 Wasserpotential [Mittelwert $(\bar{x})$ und Standartabweichung $\left(\sigma_{\mathrm{x}}\right)$ ] $(\mathrm{MPa})$ in Sorten und Knollen verschiedener spezifischer Dichten $\left(\mathrm{kg} \mathrm{L}^{-1}\right)$ nach der Ernte 2006 und nach fünf und acht Monaten Lagerung

\begin{tabular}{|c|c|c|c|c|c|c|c|c|c|c|c|c|c|c|c|c|c|c|c|}
\hline Jahr & Stadium & & spezifische Dichte & $\bar{x}$ & $\sigma_{\mathrm{x}}$ & $\bar{x}$ & $\sigma_{\mathrm{x}}$ & $\bar{x}$ & $\sigma_{\mathrm{x}}$ & $\bar{x}$ & $\sigma_{\mathrm{x}}$ & $\bar{x}$ & $\sigma_{\mathrm{x}}$ & $\bar{x}$ & $\sigma_{\mathrm{x}}$ & $\bar{x}$ & $\sigma_{\mathrm{x}}$ & $\bar{x}$ & $\sigma_{\mathrm{x}}$ \\
\hline & & & & Adretta & a $a$ & Afra & a $a$ & Gala & a $a$ & Granola & a $a$ & Lolita & b $a$ & Marabel & c $a$ & Nicola & a $a$ & Renate & b $a$ \\
\hline & & & $<1,055$ & & & & & & & & & & & & & & & & \\
\hline & & & $1,056-1,065 \mathrm{ab}$ & & & & & $-0,59$ & 0,03 & $-0,63$ & 0,06 & $-0,78$ & 0,08 & $-0,96$ & 0,14 & $-0,66$ & 0,06 & $-0,82$ & 0,07 \\
\hline & Ernte & $\mathrm{A}$ & $1,066-1,075 \mathrm{ab}$ & & & $-0,53$ & 0,05 & $-0,72$ & 0,02 & $-0,62$ & 0,06 & $-0,77$ & 0,06 & $-0,80$ & 0,11 & $-0,72$ & 0,10 & $-0,66$ & 0,05 \\
\hline & & & $1,076-1,085 \mathrm{ab}$ & $-0,71$ & 0,04 & $-0,67$ & 0,05 & & & & & & & & & $-0,66$ & 0,05 & $-0,87$ & 0,08 \\
\hline & & & $1,086-1,095$ a & $-0,64$ & 0,07 & $-0,58$ & 0,02 & & & & & & & & & & & & \\
\hline & & & $>1,095$ & & & & & & & & & & & & & & & & \\
\hline & & & & Adretta & bc $b$ & Afra & a $b$ & Gala & bc $b$ & Granola & b $a b$ & Lolita & $\mathbf{d} b$ & Marabel & de $a$ & Nicola & c $b$ & Renate & e $b$ \\
\hline & & & $<1,055$ & & & & & $-0,88$ & 0,04 & & & & & & & & & & \\
\hline & & & $1,056-1,065$ a & $-0,83$ & 0,05 & & & $-0,87$ & 0,03 & $-0,82$ & 0,08 & $-0,91$ & 0,03 & $-1,11$ & 0,03 & $-0,88$ & 0,03 & $-0,93$ & 0,02 \\
\hline \multirow[t]{11}{*}{$2006 \mathbf{B}$} & 5 Monate Lagerung & $\mathrm{B}$ & $1,066-1,075$ a & $-0,82$ & 0,03 & $-0,75$ & 0,02 & $-0,91$ & 0,07 & $-0,85$ & 0,08 & $-0,97$ & 0,04 & $-1,03$ & 0,03 & $-1,00$ & 0,04 & $-0,96$ & 0,08 \\
\hline & & & $1,076-1,085 \mathrm{~b}$ & $-0,94$ & 0,03 & $-0,76$ & 0,08 & $-0,98$ & 0,02 & $-0,92$ & 0,07 & $-1,01$ & 0,06 & $-1,05$ & 0,03 & $-0,99$ & 0,11 & $-1,27$ & 0,11 \\
\hline & & & $1,086-1,095 \mathrm{~b}$ & $-0,91$ & 0,07 & $-0,72$ & 0,03 & & & & & $-1,19$ & 0,13 & & & $-0,89$ & 0,04 & $-1,18$ & 0,07 \\
\hline & & & $>1,095$ & $-0,93$ & 0,05 & $-0,81$ & 0,06 & & & & & & & & & & & & \\
\hline & & & & Adretta & $\mathbf{a b} b$ & Afra & a $b$ & Gala & bc $b$ & Granola & bc $b$ & Lolita & bc $b$ & Marabel & bc $a$ & Nicola & $\mathbf{a b} b$ & Renate & c $b$ \\
\hline & & & $<1,055$ & & & & & $-1,07$ & 0,07 & & & & & & & $-0,91$ & 0,02 & $-0,94$ & 0,09 \\
\hline & & & $1,056-1,065$ a & $-0,87$ & 0,08 & & & $-0,91$ & 0,05 & $-0,88$ & 0,16 & $-0,98$ & 0,17 & $-0,92$ & 0,13 & $-0,92$ & 0,06 & $-0,99$ & 0,17 \\
\hline & 8 Monate Lagerung & $\mathrm{B}$ & $1,066-1,075$ a & $-0,86$ & 0,08 & $-0,72$ & 0,05 & $-0,92$ & 0,10 & $-0,80$ & 0,02 & $-0,98$ & 0,09 & $-1,04$ & 0,15 & $-0,95$ & 0,06 & $-1,15$ & 0,34 \\
\hline & & & $1,076-1,085 \mathrm{ab}$ & $-0,90$ & 0,07 & $-0,76$ & 0,04 & & & $-1,09$ & 0,14 & $-1,06$ & 0,05 & $-1,08$ & 0,06 & & & $-1,21$ & 0,18 \\
\hline & & & $1,086-1,095 \mathrm{~b}$ & $-1,04$ & 0,06 & $-0,83$ & 0,06 & & & $-1,31$ & 0,36 & $-1,05$ & 0,08 & $-1,22$ & 0,14 & & & $-1,29$ & 0,22 \\
\hline & & & $>1,095$ & & & $-0,80$ & 0,05 & & & & & & & & & & & & \\
\hline
\end{tabular}


Tabelle 10.15 Wasserpotential [Mittelwert $(\bar{x})$ und Standartabweichung $\left(\sigma_{\mathrm{x}}\right)$ ] $(\mathrm{MPa})$ in Sorten und Knollen verschiedener spezifischer Dichten $\left(\mathrm{kg} \mathrm{L}^{-1}\right)$ nach der Ernte 2007 und nach fünf und acht Monaten Lagerung

\begin{tabular}{|c|c|c|c|c|c|c|c|c|c|c|c|c|c|c|c|c|c|c|c|c|}
\hline Jahr & Stadium & & spezifische D & Dichte & $\bar{x}$ & $\sigma_{\mathrm{x}}$ & $\bar{x}$ & $\sigma_{\mathrm{x}}$ & $\bar{x}$ & $\sigma_{\mathrm{x}}$ & $\bar{x}$ & $\sigma_{\mathrm{x}}$ & $\bar{x}$ & $\sigma_{\mathrm{x}}$ & $\bar{x}$ & $\sigma_{\mathrm{x}}$ & $\bar{x}$ & $\sigma_{\mathrm{x}}$ & $\bar{x}$ & $\sigma_{\mathrm{x}}$ \\
\hline & & & & & Adretta & a $a$ & Afra & $\mathbf{a b} a$ & Gala & $\mathbf{a b} a$ & Granola & ab $a$ & Lolita & b $a$ & Marabel & c $a$ & Nicola & c $a$ & Renate & ab $a$ \\
\hline & & & $<1,055$ & $\mathrm{a}$ & & & & & $-0,65$ & 0,07 & & & & & $-0,79$ & 0,12 & & & & \\
\hline & & & $1,056-1,065$ & $\mathrm{a}$ & $-0,56$ & 0,05 & & & $-0,67$ & 0,05 & $-0,73$ & 0,02 & $-0,77$ & 0,07 & $-0,79$ & 0,04 & $-0,78$ & 0,04 & $-0,72$ & 0,05 \\
\hline & Ernte & A & $1,066-1,075$ & $\mathrm{a}$ & $-0,64$ & 0,02 & $-0,71$ & 0,02 & $-0,75$ & 0,03 & $-0,68$ & 0,04 & $-0,67$ & 0,04 & $-0,79$ & 0,05 & $-0,71$ & 0,05 & $-0,70$ & 0,04 \\
\hline & & & $1,076-1,085$ & $\mathrm{a}$ & $-0,63$ & 0,04 & $-0,67$ & 0,04 & $-0,72$ & 0,06 & $-0,69$ & 0,03 & $-0,73$ & 0,06 & $-0,78$ & 0,04 & $-0,87$ & 0,04 & $-0,72$ & 0,04 \\
\hline & & & $1,086-1,095$ & $\mathrm{a}$ & $-0,74$ & 0,05 & $-0,69$ & 0,03 & & & & & & & & & & & & \\
\hline & & & $>1,095$ & $\mathrm{a}$ & $-0,74$ & 0,02 & & & & & & & & & & & & & & \\
\hline & & & & & Adretta & bc $b$ & Afra & a $a b$ & Gala & $\mathbf{a b} b$ & Granola & a $a$ & Lolita & $\mathbf{c d} b$ & Marabel & $\mathbf{d} b$ & Nicola & $\mathbf{d} a b$ & Renate & cd $a b$ \\
\hline & & & $<1,055$ & $\mathrm{a}$ & & & & & $-0,80$ & 0,04 & & & & & & & & & & \\
\hline & & & $1,056-1,065$ & $\mathrm{a}$ & & & & & $-0,75$ & 0,05 & $-0,77$ & 0,03 & $-0,82$ & 0,05 & $-0,88$ & 0,07 & $-0,81$ & 0,02 & $-0,83$ & 0,03 \\
\hline \multirow[t]{11}{*}{2007 A } & 5 Monate Lagerung & B & $1,066-1,075$ & $\mathrm{a}$ & $-0,83$ & 0,02 & $-0,68$ & 0,09 & $-0,77$ & 0,03 & $-0,74$ & 0,02 & $-0,90$ & 0,03 & $-0,89$ & 0,05 & $-0,87$ & 0,05 & $-0,83$ & 0,03 \\
\hline & & & $1,076-1,085$ & $\mathrm{a}$ & $-0,79$ & 0,01 & $-0,76$ & 0,03 & $-0,85$ & 0,12 & $-0,75$ & 0,05 & $-0,86$ & 0,03 & $-0,86$ & 0,07 & $-0,82$ & 0,04 & $-0,83$ & 0,00 \\
\hline & & & $1,086-1,095$ & $\mathrm{a}$ & $-0,80$ & 0,03 & $-0,77$ & 0,02 & & & $-0,74$ & 0,04 & $-0,82$ & 0,01 & & & $-0,97$ & 0,06 & $-0,88$ & 0,04 \\
\hline & & & $>1,095$ & $\mathrm{a}$ & $-0,86$ & 0,02 & $-0,76$ & 0,02 & & & & & & & & & & & & \\
\hline & & & & & Adretta & $\mathbf{b} c$ & Afra & $\mathbf{a} a b$ & Gala & ab $c$ & Granola & $\mathbf{a b} b$ & Lolita & ab $b$ & Marabel & b $b$ & Nicola & b $b$ & Renate & b $a b$ \\
\hline & & & $<1,055$ & $\mathrm{a}$ & & & & & $-0,90$ & 0,06 & $-0,93$ & 0,16 & & & & & & & & \\
\hline & & & $1,056-1,065$ & $\mathrm{a}$ & $-0,88$ & 0,02 & & & $-0,86$ & 0,06 & $-0,87$ & 0,05 & $-0,87$ & 0,07 & $-0,93$ & 0,05 & $-0,92$ & 0,08 & $-1,00$ & 0,26 \\
\hline & 8 Monate Lagerung & $\mathrm{C}$ & $1,066-1,075$ & $\mathrm{a}$ & $-0,92$ & 0,03 & $-0,89$ & 0,05 & $-0,95$ & 0,02 & $-0,85$ & 0,06 & $-0,89$ & 0,11 & $-0,96$ & 0,07 & $-0,95$ & 0,05 & $-0,84$ & 0,05 \\
\hline & & & $1,076-1,085$ & $\mathrm{a}$ & $-0,85$ & 0,10 & $-0,74$ & 0,07 & $-0,90$ & 0,05 & $-0,84$ & 0,05 & $-0,86$ & 0,08 & $-0,97$ & 0,04 & $-0,94$ & 0,05 & $-0,92$ & 0,07 \\
\hline & & & 1,086-1,095 & $\mathrm{a}$ & $-0,91$ & 0,08 & $-0,80$ & 0,05 & & & & & & & & & $-0,95$ & 0,09 & & \\
\hline & & & $>1,095$ & $\mathrm{a}$ & $-1,09$ & 0,05 & $-0,81$ & 0,10 & & & & & & & & & & & & \\
\hline
\end{tabular}


Tabelle 10.16 Wasserpotential [Mittelwert $(\bar{x})$ und Standartabweichung $\left(\sigma_{\mathrm{x}}\right)$ ] $(\mathrm{MPa})$ in Kronen- und Nabelgewebe nach der Ernte 2006 und 2007 und nach fünf und acht Monaten Lagerung der Sorten, deren Knollen nicht nach spezifischen Dichten getrennt wurden

\begin{tabular}{|c|c|c|c|c|c|c|c|c|c|c|c|c|c|c|c|c|c|c|}
\hline Jahr & Stadium & Gewebe & $\bar{x}$ & $\sigma_{\mathrm{x}}$ & $\bar{x}$ & $\sigma_{\mathrm{x}}$ & $\bar{x}$ & $\sigma_{\mathrm{x}}$ & $\bar{x}$ & $\sigma_{\mathrm{x}}$ & $\bar{x}$ & $\sigma_{\mathrm{x}}$ & $\bar{x}$ & $\sigma_{\mathrm{x}}$ & $\bar{x}$ & $\sigma_{\mathrm{x}}$ & $\bar{x}$ & $\sigma_{\mathrm{x}}$ \\
\hline \multirow{9}{*}{2006 A } & \multirow{3}{*}{ Ernte } & & \multicolumn{2}{|c|}{ Adretta a $a$} & \multicolumn{2}{|c|}{ Afra a $a$} & \multicolumn{2}{|c|}{ Gala a $a$} & \multicolumn{2}{|c|}{ Granola a $a$} & \multicolumn{2}{|c|}{ Lolita a $a$} & \multicolumn{2}{|c|}{ Marabel a $a$} & \multicolumn{2}{|c|}{ Nicola a $a$} & \multicolumn{2}{|c|}{ Renate a $a$} \\
\hline & & Nabel a & $-0,56$ & 0,13 & $-0,69$ & 0,02 & $-0,74$ & 0,01 & $-0,70$ & 0,09 & $-0,75$ & 0,06 & $-0,70$ & 0,04 & $-0,71$ & 0,05 & $-0,68$ & 0,10 \\
\hline & & Krone a & $-0,59$ & 0,02 & $-0,57$ & 0,05 & $-0,69$ & 0,03 & $-0,64$ & 0,09 & $-0,69$ & 0,05 & $-0,75$ & 0,10 & $-0,72$ & 0,06 & $-0,61$ & 0,01 \\
\hline & & & \multicolumn{2}{|c|}{ Adretta a $a$} & \multicolumn{2}{|c|}{ Afra a $a$} & \multicolumn{2}{|c|}{ Gala a $a$} & \multicolumn{2}{|c|}{ Granola ab $a$} & \multicolumn{2}{|c|}{ Lolita $\mathbf{b} a$} & \multicolumn{2}{|c|}{ Marabel $\mathbf{b} b$} & \multicolumn{2}{|c|}{ Nicola $\mathbf{a b} a$} & \multicolumn{2}{|c|}{ Renate b $a$} \\
\hline & 5 Monate Lagerung $\mathrm{B}$ & Nabel a & $-0,77$ & 0,04 & $-0,72$ & 0,02 & $-0,87$ & 0,03 & $-0,82$ & 0,01 & $-0,99$ & 0,06 & $-1,05$ & 0,02 & $-0,92$ & 0,11 & $-0,97$ & 0,07 \\
\hline & & Krone a & $-0,64$ & 0,05 & $-0,79$ & 0,06 & $-0,84$ & 0,04 & $-0,83$ & 0,02 & $-1,06$ & 0,10 & $-1,04$ & 0,05 & $-0,90$ & 0,02 & $-1,09$ & 0,15 \\
\hline & & & \multicolumn{2}{|c|}{ Adretta a $a$} & \multicolumn{2}{|c|}{ Afra a $a$} & \multicolumn{2}{|c|}{ Gala a $a$} & \multicolumn{2}{|c|}{ Granola a $a$} & \multicolumn{2}{|c|}{ Lolita a $a$} & \multicolumn{2}{|c|}{ Marabel a $b$} & \multicolumn{2}{|c|}{ Nicola a $a$} & \multicolumn{2}{|c|}{ Renate a $a$} \\
\hline & \multirow[t]{2}{*}{8 Monate Lagerung B } & Nabel a & $-1,02$ & 0,07 & $-0,82$ & 0,01 & $-0,86$ & 0,01 & $-0,97$ & 0,08 & $-0,84$ & 0,06 & $-1,08$ & 0,03 & $-0,89$ & 0,01 & $-0,90$ & 0,05 \\
\hline & & Krone a & $-0,86$ & 0,12 & $-0,79$ & 0,08 & $-0,86$ & 0,15 & $-0,81$ & 0,14 & $-0,89$ & 0,04 & $-1,13$ & 0,07 & $-0,88$ & 0,10 & $-0,90$ & 0,04 \\
\hline & & & Adretta & $a$ & Afra & b $a$ & Gala & b $a$ & Granola & ab $a$ & Lolita & $\mathbf{a b} a$ & Marab & b $a$ & Nicola & ab $a$ & Renate & ab $a$ \\
\hline & Ernte & Nabel a & $-0,59$ & 0,01 & $-0,73$ & 0,01 & $-0,64$ & 0,03 & $-0,67$ & 0,03 & $-0,66$ & 0,01 & $-0,81$ & 0,04 & $-0,75$ & 0,05 & $-0,71$ & 0,05 \\
\hline & & Krone a & $-0,63$ & 0,01 & $-0,71$ & 0,01 & $-0,69$ & 0,04 & $-0,70$ & 0,02 & $-0,66$ & 0,03 & $-0,80$ & 0,02 & $-0,75$ & 0,08 & $-0,72$ & 0,07 \\
\hline & & & Adretta & $\mathbf{a b} b$ & Afra & & Gala & $a b$ & Granola & a $a$ & Lolita & ab $a$ & Marab & b $a$ & Nicola & ab $a$ & Renate & ab $a$ \\
\hline \multirow[t]{5}{*}{2007 A } & 5 Monate Lagerung B & Nabel a & $-0,76$ & 0,02 & $-0,71$ & 0,07 & $-0,78$ & 0,03 & $-0,73$ & 0,02 & $-0,85$ & 0,02 & $-0,87$ & 0,03 & $-0,86$ & 0,03 & $-0,77$ & 0,02 \\
\hline & & Krone a & $-0,79$ & 0,03 & $-0,80$ & 0,01 & $-0,74$ & 0,03 & $-0,73$ & 0,03 & $-0,81$ & 0,02 & $-0,94$ & 0,00 & $-0,82$ & 0,07 & $-0,85$ & 0,03 \\
\hline & & & \multicolumn{2}{|c|}{ Adretta a $c$} & Afra & & Gala & $b$ & Granoli & $\mathbf{a} b$ & Lolita & $a$ & Marab & a $a$ & Nicola & $a$ & Renate & a $a$ \\
\hline & 8 Monate Lagerung C & Nabel a & $-0,88$ & 0,00 & $-0,78$ & 0,04 & $-0,85$ & 0,03 & $-0,92$ & 0,02 & $-0,87$ & 0,03 & $-0,94$ & 0,04 & $-0,94$ & 0,01 & $-0,90$ & 0,07 \\
\hline & & Krone a & $-0,88$ & 0,02 & $-0,77$ & 0,11 & $-0,84$ & 0,03 & $-0,84$ & 0,01 & $-0,81$ & 0,16 & $-0,91$ & 0,10 & $-0,95$ & 0,14 & $-0,96$ & 0,04 \\
\hline
\end{tabular}


Tabelle 10.17 Osmolalität [Mittelwert $(\bar{x})$ und Standartabweichung $\left(\sigma_{\mathrm{x}}\right)$ ] $\left(\mathrm{mmol} \mathrm{kg}^{-1}\right)$ in Sorten und Knollen verschiedener spezifischer Dichten $\left(\mathrm{kg} \mathrm{L}^{-1}\right)$ nach der Ernte 2006 und nach fünf und acht Monaten Lagerung

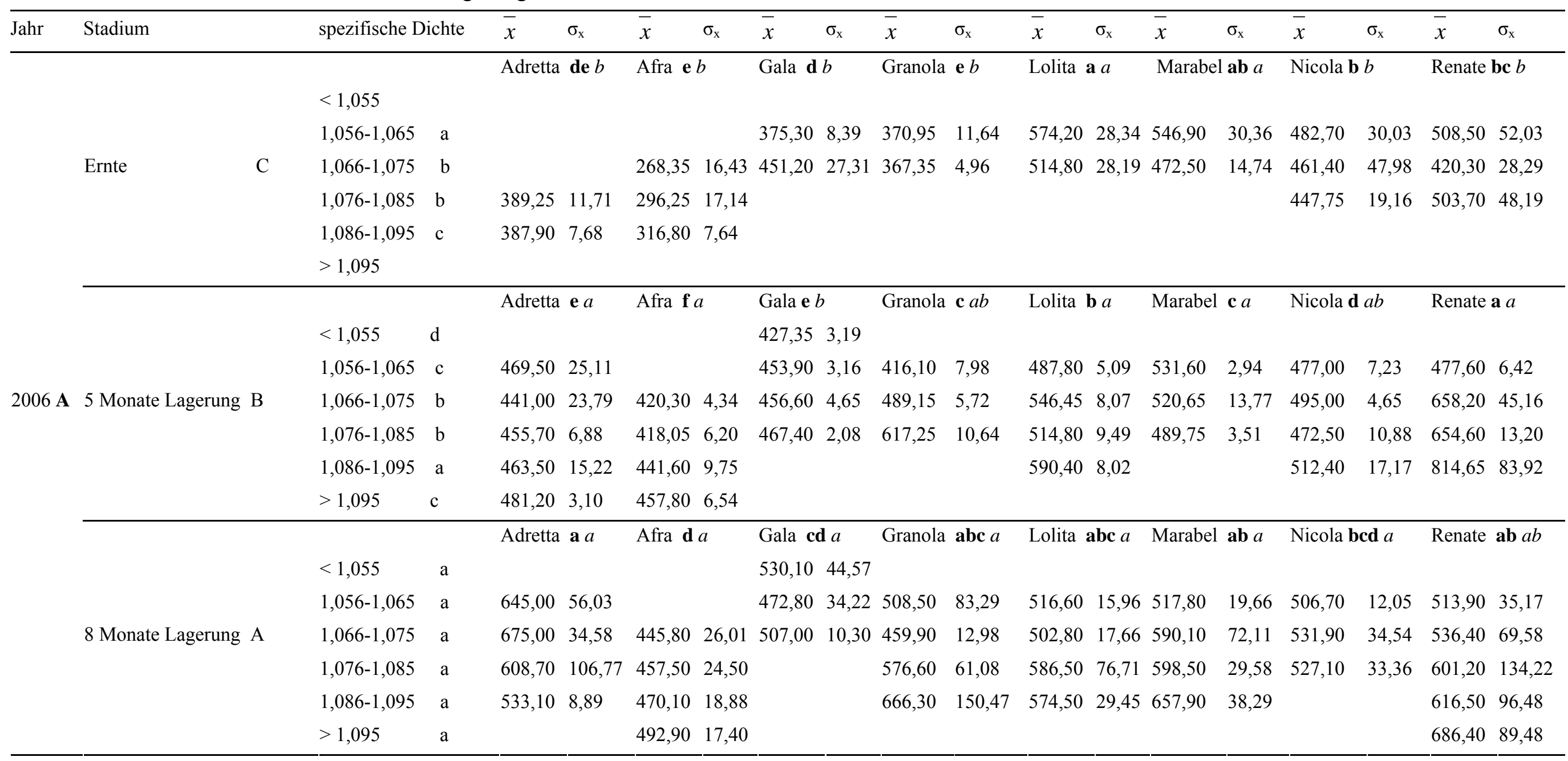


Tabelle 10.18 Osmolalität [Mittelwert $(\bar{x})$ und Standartabweichung $\left(\sigma_{\mathrm{x}}\right)$ ] $\left(\mathrm{mmol} \mathrm{kg}^{-1}\right)$ in Sorten und Knollen verschiedener spezifischer Dichten $\left(\mathrm{kg} \mathrm{L}^{-1}\right)$ nach der Ernte 2007 und nach fünf und acht Monaten Lagerung

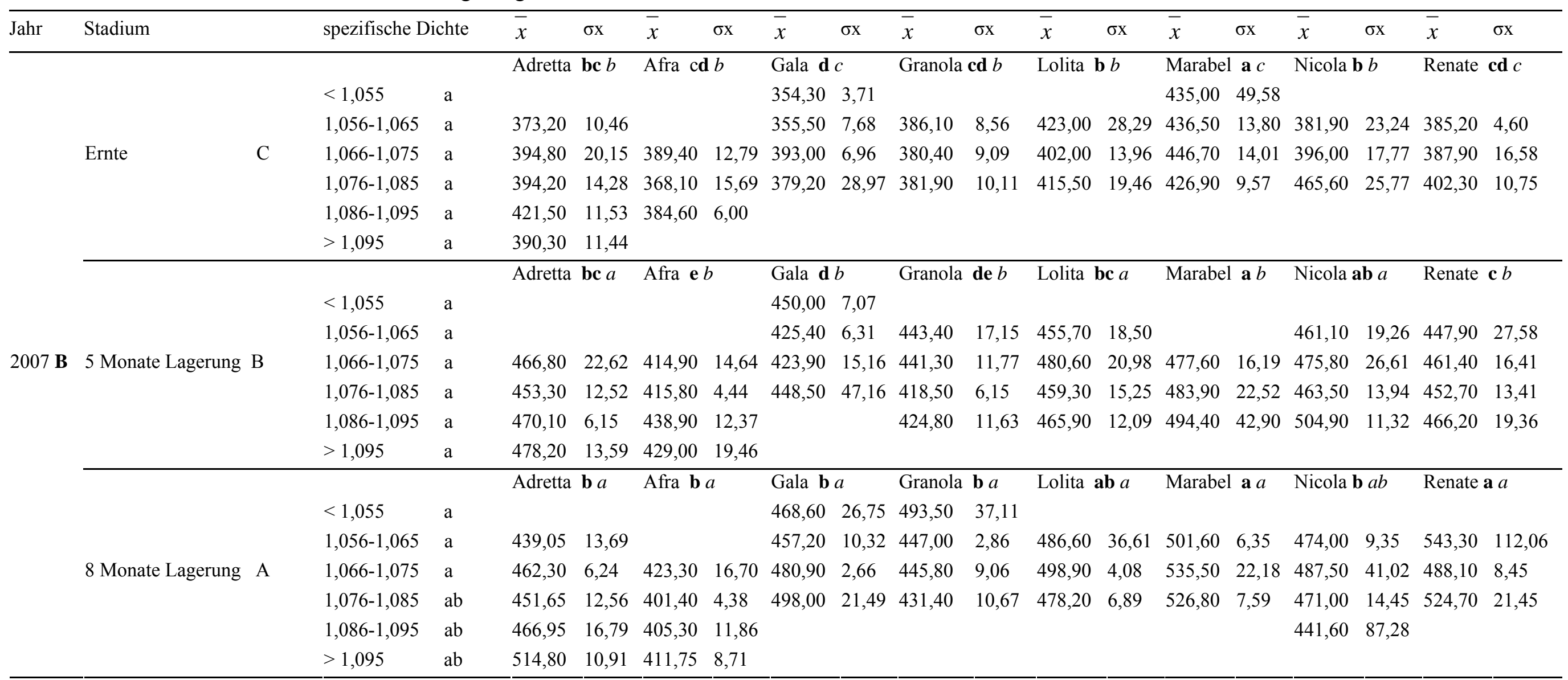


Tabelle 10.19 Osmolalität [Mittelwert $(\bar{x})$ und Standartabweichung $\left(\sigma_{\mathrm{x}}\right)$ ] $\left(\mathrm{mmol} \mathrm{kg}^{-1}\right)$ in Kronen- und Nabelgewebe nach der Ernte 2006 und 2007 und nach fünf und acht Monaten Lagerung der Sorten, deren Knollen nicht nach spezifischen Dichten getrennt wurden

\begin{tabular}{|c|c|c|c|c|c|c|c|c|c|c|c|c|c|c|c|c|c|c|c|}
\hline Jahr & Stadium & & Gewebe & $\bar{x}$ & $\sigma_{\mathrm{x}}$ & $\bar{x}$ & $\sigma_{\mathrm{x}}$ & $\bar{x}$ & $\sigma_{\mathrm{x}}$ & $\bar{x}$ & $\sigma_{\mathrm{x}}$ & $\bar{x}$ & $\sigma_{\mathrm{x}}$ & $\bar{x}$ & $\sigma_{\mathrm{x}}$ & $\bar{x}$ & $\sigma_{\mathrm{x}}$ & $\bar{x}$ & $\sigma_{\mathrm{x}}$ \\
\hline \multirow{9}{*}{2006 A } & \multirow{3}{*}{ Ernte } & \multirow{3}{*}{$\mathrm{B}$} & & \multicolumn{2}{|c|}{ Adretta $\mathbf{b} b$} & \multicolumn{2}{|c|}{ Afra $\mathbf{a b} a$} & \multicolumn{2}{|c|}{ Gala $\mathbf{a b} b$} & \multicolumn{2}{|c|}{ Granola $\mathbf{a b} b$} & \multicolumn{2}{|c|}{ Lolita $\mathbf{a b} a$} & \multicolumn{2}{|c|}{ Marabel a $b$} & \multicolumn{2}{|c|}{ Nicola $\mathbf{a b} a$} & \multicolumn{2}{|c|}{ Renate ab $a$} \\
\hline & & & Nabel a & 345,90 & 7,21 & 400,80 & 1,69 & 394,80 & 6,79 & 368,40 & 11,88 & 477,00 & 26,30 & 485,40 & 22,91 & 449,40 & 44,97 & 419,40 & 7,64 \\
\hline & & & Krone a & 373,50 & 19,09 & 340,80 & 78,07 & 406,80 & 6,79 & 374,10 & 9,76 & 423,60 & 13,58 & 444,00 & 1,69 & 463,20 & 5,09 & 441,60 & 16,12 \\
\hline & & & & \multicolumn{2}{|c|}{ Adretta e $a$} & \multicolumn{2}{|l|}{ Afra $\mathbf{f} a$} & \multicolumn{2}{|c|}{ Gala e $a$} & \multicolumn{2}{|c|}{ Granola $\mathbf{f} b$} & \multicolumn{2}{|c|}{ Lolita $\mathbf{b} a$} & \multicolumn{2}{|c|}{ Marabel a $a$} & \multicolumn{2}{|c|}{ Nicola c $a$} & \multicolumn{2}{|c|}{ Renate $\mathbf{d} a$} \\
\hline & 5 Monate Lagerung & A & Nabel a & 456,00 & 5,09 & 420,90 & 1,27 & 456,60 & 2,55 & 412,20 & 0,85 & 516,00 & 5,09 & 576,60 & 2,55 & 502,50 & 2,12 & 484,80 & 0,00 \\
\hline & & & Krone a & 468,90 & 15,69 & 418,50 & 0,42 & 473,40 & 5,94 & 415,80 & 0,85 & 539,40 & 7,64 & 586,20 & 0,85 & 508,80 & 5,09 & 487,80 & 2,55 \\
\hline & & & & \multicolumn{2}{|c|}{ Adretta $\mathbf{b} a$} & \multicolumn{2}{|l|}{ Afra $\mathbf{b} a$} & \multicolumn{2}{|c|}{ Gala b $a$} & \multicolumn{2}{|c|}{ Granola $\mathbf{b} a$} & \multicolumn{2}{|c|}{ Lolita $\mathbf{b} a$} & \multicolumn{2}{|c|}{ Marabel a $a$} & \multicolumn{2}{|c|}{ Nicola $\mathbf{a b} a$} & \multicolumn{2}{|c|}{ Renate ab $a$} \\
\hline & 8 Monate Lagerung & A & Nabel a & 487,20 & 6,79 & 460,80 & 0,00 & 458,40 & 3,39 & 481,20 & 20,37 & 478,80 & 22,06 & 564,00 & 5,09 & 495,00 & 19,52 & 489,60 & 47,52 \\
\hline & & & Krone a & 472,20 & 9,33 & 473,40 & 12,73 & 478,80 & 0,00 & 474,60 & 11,03 & 501,60 & 39,03 & 592,20 & 12,73 & 513,00 & 9,33 & 534,00 & 11,88 \\
\hline & & & & Adretta & a $a$ & Afra a $b$ & & Gala a $t$ & & Granola & a $b$ & Lolita a & & Marabel & a $b$ & Nicola & $b$ & Renate & a $a$ \\
\hline & Ernte & $\mathrm{B}$ & Nabel a & 402,00 & 3,39 & 373,80 & 2,55 & 369,00 & 0,85 & 380,40 & 3,39 & 396,60 & 5,94 & 414,60 & 33,09 & 394,20 & 22,91 & 390,00 & 6,79 \\
\hline & & & Krone a & 405,60 & 16,97 & 372,00 & 15,27 & 360,00 & 3,39 & 355,20 & 1,69 & 372,60 & 7,64 & 408,00 & 11,88 & 385,20 & 18,67 & 387,60 & 27,15 \\
\hline & & & & Adretta & b $a$ & Afra $\mathbf{b} a$ & & Gala b & & Granola & b $a$ & Lolita $\mathbf{b}$ & $a b$ & Marabel & $a b$ & Nicola & b $a b$ & Renate & $\mathbf{a b} a$ \\
\hline \multirow[t]{5}{*}{$2007 \mathbf{B}$} & 5 Monate Lagerung & A & Nabel a & 440,40 & 0,00 & 412,80 & 5,09 & 423,00 & 11,03 & 454,20 & 5,94 & 453,60 & 15,27 & 482,40 & 5,09 & 450,00 & 0,00 & 432,00 & 5,09 \\
\hline & & & Krone a & 453,60 & 18,67 & 450,60 & 4,24 & 429,00 & 0,85 & 430,20 & 9,33 & 428,40 & 3,39 & 497,40 & 4,24 & 462,60 & 2,55 & 479,40 & 17,82 \\
\hline & & & & \multicolumn{2}{|c|}{ Adretta $\mathbf{a b} a$} & \multicolumn{2}{|c|}{ Afra $\mathbf{b} a b$} & Gala ac & & Granola & ab $a$ & Lolita al & & Marabel & a $a$ & Nicola & b $a$ & Renate & ab $a$ \\
\hline & 8 Monate Lagerung & A & Nabel a & 445,20 & 20,37 & 399,90 & 10,61 & 474,60 & 22,91 & 440,40 & 18,67 & 463,80 & 26,30 & 494,40 & 11,88 & 479,40 & 4,24 & 448,20 & 17,82 \\
\hline & & & Krone a & 438,90 & 14,85 & 385,80 & 7,64 & 497,40 & 50,06 & 448,20 & 19,52 & 474,00 & 10,18 & 501,00 & 5,94 & 471,60 & 10,18 & 446,40 & 45,82 \\
\hline
\end{tabular}


Tabelle 10.20 Osmotisches Potential [Mittelwert $(\bar{x})$ und Standartabweichung $\left(\sigma_{\mathrm{x}}\right)$ ] $(\mathrm{MPa})$ in Sorten und Knollen verschiedener spezifischer Dichten $\left(\mathrm{kg} \mathrm{L}^{-1}\right)$ nach der Ernte 2006 und nach fünf und acht Monaten Lagerung

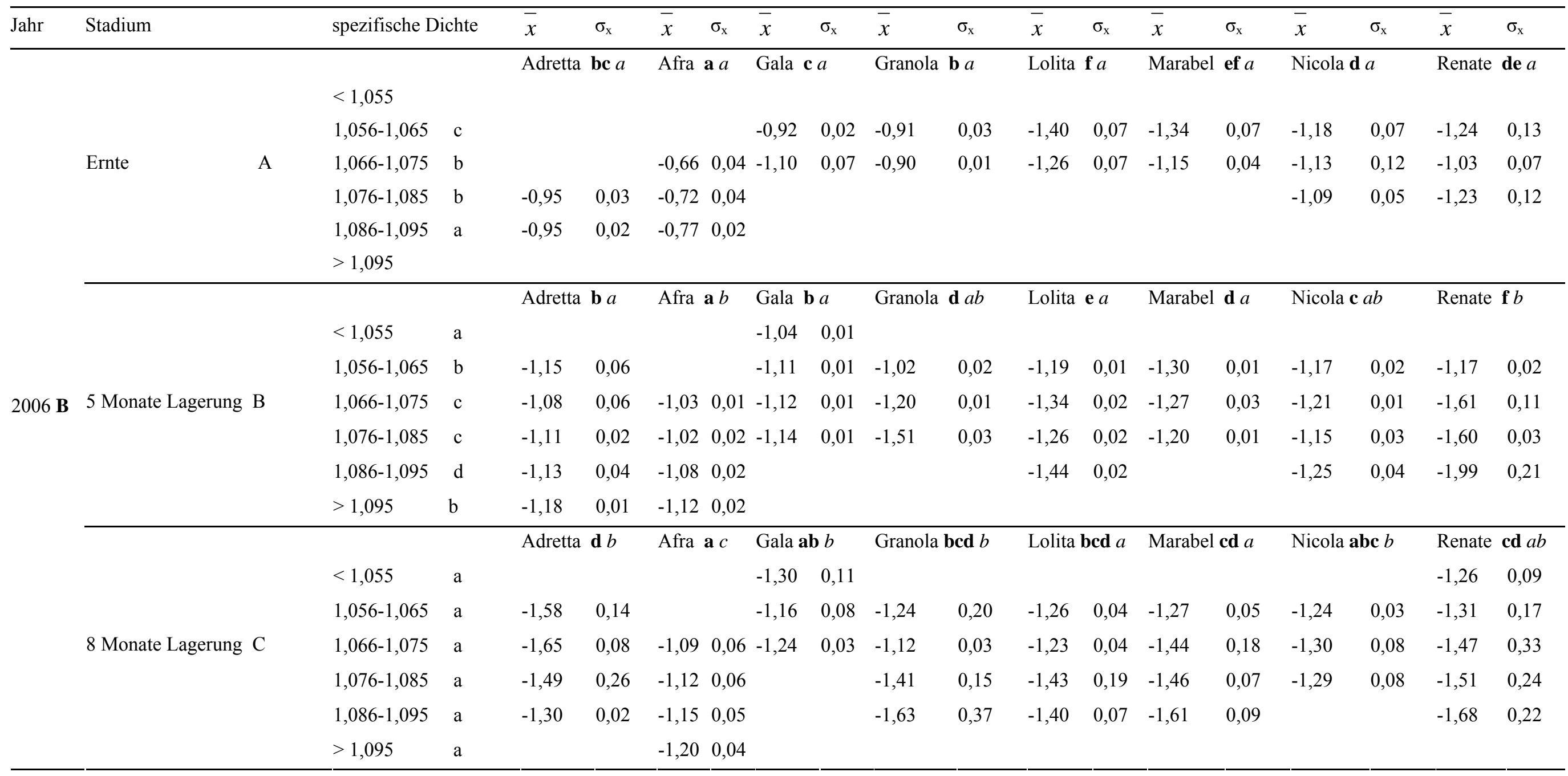


Tabelle 10.21 Osmotisches Potential [Mittelwert $(\bar{x})$ und Standartabweichung $\left(\sigma_{\mathrm{x}}\right)$ ] $(\mathrm{MPa})$ in Sorten und Knollen verschiedener spezifischer Dichten $\left(\mathrm{kg} \mathrm{L}^{-1}\right)$ nach der Ernte 2007 und nach fünf und acht Monaten Lagerung

\begin{tabular}{|c|c|c|c|c|c|c|c|c|c|c|c|c|c|c|c|c|c|c|c|c|}
\hline Jahr & \multicolumn{2}{|l|}{ Stadium } & \multicolumn{2}{|c|}{ spezifische Dichte } & \multicolumn{2}{|l|}{$\bar{x}$} & \multirow{2}{*}{$\frac{\bar{x}}{\text { Afra }}$} & \multirow{2}{*}{$\frac{\sigma_{\mathrm{x}}}{\mathbf{a b} a}$} & \multirow{2}{*}{$\frac{\bar{x}}{\text { Gala }}$} & \multirow{2}{*}{$\frac{\sigma_{\mathrm{x}}}{\mathbf{a} a}$} & \multirow{2}{*}{$\frac{\bar{x}}{\text { Granola }}$} & & \multirow{2}{*}{$\frac{\bar{x}}{\text { Lolita }}$} & \multirow{2}{*}{$\frac{\sigma_{\mathrm{x}}}{\mathbf{c} a}$} & \multirow{2}{*}{\multicolumn{2}{|c|}{$\frac{\bar{x} \quad \sigma_{\mathrm{x}}}{\text { Marabel } \mathbf{d} a}$}} & \multirow{2}{*}{\multicolumn{2}{|c|}{$\begin{array}{l}\bar{x} \quad \sigma_{\mathrm{x}} \\
\text { Nicola c } a\end{array}$}} & \multirow{2}{*}{$\frac{\bar{x}}{\text { Renate }}$} & \multirow{2}{*}{$\frac{\sigma_{\mathrm{x}}}{\mathbf{a b} a}$} \\
\hline & & & & & Adrett & bc $a$ & & & & & & & & & & & & & & \\
\hline & & & $<1,055$ & $\mathrm{a}$ & & & & & $-0,87$ & 0,01 & & & & & $-1,06$ & 0,12 & & & & \\
\hline & \multirow{5}{*}{ Ernte } & & $1,056-1,065$ & $\mathrm{a}$ & $-0,91$ & 0,03 & & & $-0,87$ & 0,02 & $-0,94$ & 0,02 & $-1,03$ & 0,07 & $-1,07$ & 0,03 & $-0,93$ & 0,06 & $-0,94$ & 0,01 \\
\hline & & A & $1,066-1,075$ & $\mathrm{a}$ & $-0,97$ & 0,05 & $-0,95$ & 0,03 & $-0,96$ & 0,02 & $-0,93$ & 0,02 & $-0,98$ & 0,03 & $-1,09$ & 0,03 & $-0,97$ & 0,04 & $-0,95$ & 0,04 \\
\hline & & & $1,076-1,085$ & $\mathrm{a}$ & $-0,96$ & 0,03 & $-0,90$ & 0,04 & $-0,93$ & 0,07 & $-0,93$ & 0,02 & $-1,02$ & 0,05 & $-1,04$ & 0,02 & $-1,14$ & 0,06 & $-0,98$ & 0,03 \\
\hline & & & $1,086-1,095$ & $\mathrm{a}$ & $-1,03$ & 0,03 & $-0,94$ & 0,01 & & & & & & & & & & & & \\
\hline & & & $>1,095$ & $\mathrm{a}$ & $-0,95$ & 0,03 & & & & & & & & & & & & & & \\
\hline & & & & & Adrett & $\mathrm{cd} a$ & Afra : & $a b$ & Gala & b $b$ & Granola & ab $b$ & Lolita & cd $b$ & Marabel & e $b$ & Nicola & de $b$ & Renate & c $b$ \\
\hline & & & $<1,055$ & $\mathrm{a}$ & & & & & $-1,10$ & 0,02 & & & & & & & & & & \\
\hline & & & $1,056-1,065$ & $\mathrm{a}$ & & & & & $-1,04$ & 0,02 & $-1,08$ & 0,04 & $-1,11$ & 0,05 & $-1,17$ & 0,04 & $-1,13$ & 0,05 & $-1,09$ & 0,07 \\
\hline \multirow[t]{11}{*}{2007 A } & 5 Monate Lagerung & B & $1,066-1,075$ & $\mathrm{a}$ & $-1,14$ & 0,06 & $-1,01$ & 0,04 & $-1,04$ & 0,04 & $-1,08$ & 0,03 & $-1,17$ & 0,05 & $-1,18$ & 0,06 & $-1,16$ & 0,07 & $-1,13$ & 0,04 \\
\hline & & & $1,076-1,085$ & $\mathrm{a}$ & $-1,11$ & 0,03 & $-1,02$ & 0,01 & $-1,10$ & 0,12 & $-1,02$ & 0,02 & $-1,12$ & 0,04 & $-1,21$ & 0,10 & $-1,13$ & 0,03 & $-1,11$ & 0,03 \\
\hline & & & $1,086-1,095$ & $\mathrm{a}$ & $-1,15$ & 0,02 & $-1,07$ & 0,03 & & & $-1,04$ & 0,03 & $-1,14$ & 0,03 & & & $-1,23$ & 0,03 & $-1,14$ & 0,05 \\
\hline & & & $>1,095$ & $\mathrm{a}$ & $-1,17$ & 0,03 & $-1,05$ & 0,05 & & & & & & & & & & & & \\
\hline & & & & & Adrett & $\mathbf{b} b$ & Afra & $a b$ & Gala & $\mathbf{b} c$ & Granola & $\mathbf{b} b$ & Lolita & bc $b$ & Marabel & $\mathbf{c} C$ & Nicola & b $b$ & Renate & $\mathbf{c} C$ \\
\hline & & & $<1,055$ & $\mathrm{~b}$ & & & & & $-1,15$ & 0,07 & $-1,21$ & 0,09 & & & & & & & & \\
\hline & & & $1,056-1,065$ & $\mathrm{~b}$ & $-1,07$ & 0,03 & & & $-1,12$ & 0,03 & $-1,09$ & 0,01 & $-1,19$ & 0,09 & $-1,23$ & 0,02 & $-1,16$ & 0,02 & $-1,33$ & 0,27 \\
\hline & 8 Monate Lagerung & $\mathrm{B}$ & $1,066-1,075$ & $\mathrm{~b}$ & $-1,13$ & 0,02 & $-1,03$ & 0,04 & $-1,18$ & 0,01 & $-1,09$ & 0,02 & $-1,22$ & 0,01 & $-1,31$ & 0,05 & $-1,19$ & 0,10 & $-1,19$ & 0,02 \\
\hline & & & $1,076-1,085$ & $a b$ & $-1,10$ & 0,03 & $-0,98$ & 0,01 & $-1,22$ & 0,05 & $-1,05$ & 0,03 & $-1,17$ & 0,02 & $-1,29$ & 0,02 & $-1,15$ & 0,04 & $-1,28$ & 0,05 \\
\hline & & & $1,086-1,095$ & $\mathrm{a}$ & $-1,14$ & 0,04 & $-0,99$ & 0,03 & & & & & & & & & $-1,08$ & 0,21 & & \\
\hline & & & $>1,095$ & $a b$ & $-1,26$ & 0,03 & $-1,01$ & 0,02 & & & & & & & & & & & & \\
\hline
\end{tabular}


Tabelle 10.22 Osmotisches Potential [Mittelwert $(\bar{x})$ und Standartabweichung $\left(\sigma_{\mathrm{x}}\right)$ ] $(\mathrm{MPa})$ in Kronen- und Nabelgewebe nach der Ernte 2006 und 2007 und nach fünf und acht Monaten Lagerung der Sorten, deren Knollen nicht nach spezifischen Dichten getrennt wurden

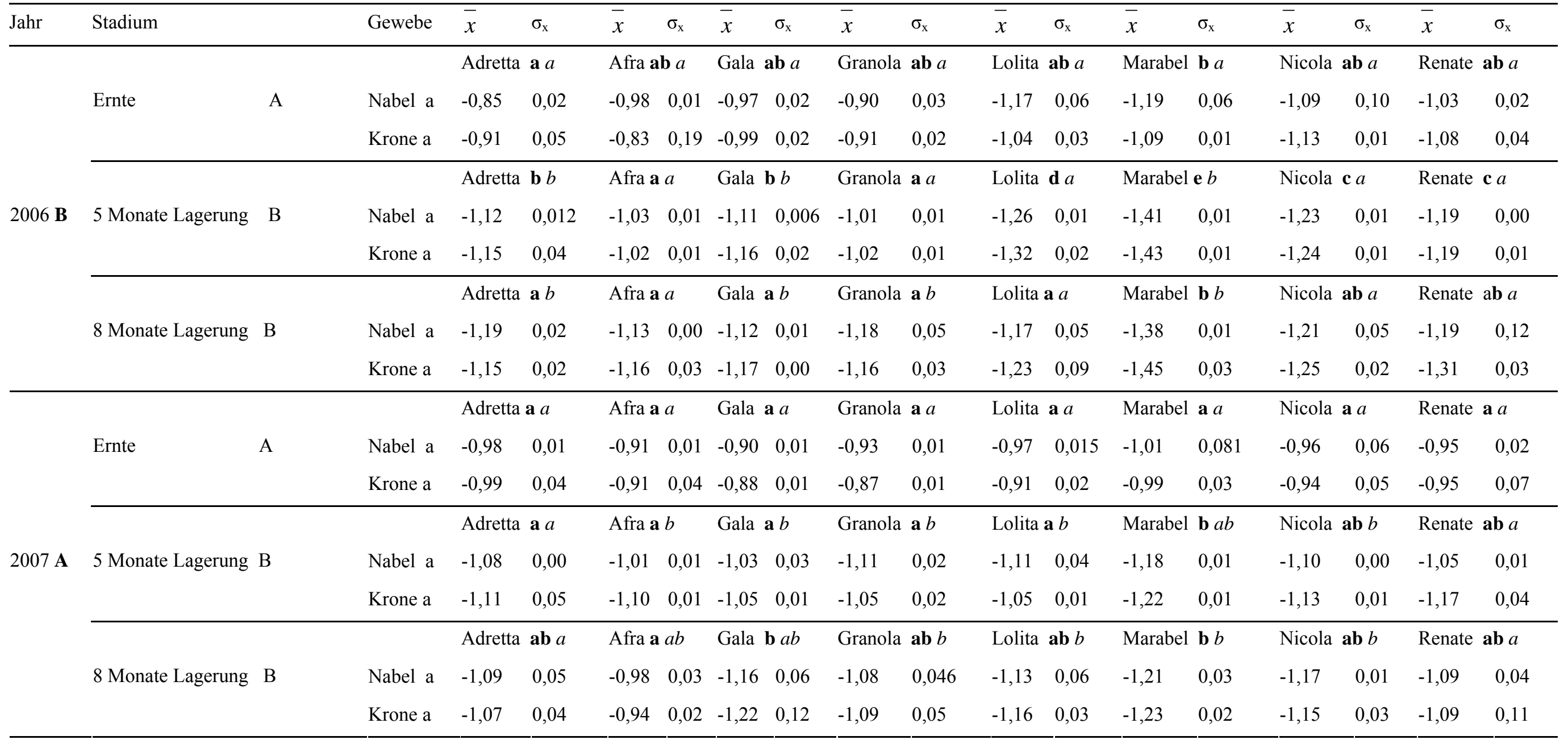


Tabelle 10.23 Binnendruck (Turgor) [Mittelwert $(\bar{x})$ und Standartabweichung $\left(\sigma_{\mathrm{x}}\right)$ ] (MPa) in Sorten und Knollen verschiedener spezifischer Dichten $\left(\mathrm{kg} \mathrm{L}^{-1}\right)$ nach der Ernte 2006 und nach fünf und acht Monaten Lagerung

\begin{tabular}{|c|c|c|c|c|c|c|c|c|c|c|c|c|c|c|c|c|c|c|c|}
\hline \multirow[t]{2}{*}{ Jahr } & Stadium & \multicolumn{2}{|c|}{ spezifische Dichte } & \multirow{2}{*}{$\begin{array}{l}x \\
\text { Adretta }\end{array}$} & & \multirow{2}{*}{$\begin{array}{l}\bar{x} \\
\text { Afra }\end{array}$} & \multirow{2}{*}{$\frac{\sigma_{\mathrm{x}}}{\mathbf{d} b}$} & & & \multirow{2}{*}{$\begin{array}{l}\bar{x} \\
\text { Granola }\end{array}$} & \multirow{2}{*}{$\begin{array}{l}\sigma_{\mathrm{x}} \\
\mathbf{c} a\end{array}$} & \multirow{2}{*}{$\begin{array}{l}\bar{x} \\
\text { Lolita }\end{array}$} & \multirow{2}{*}{$\begin{array}{l}\sigma_{\mathrm{x}} \\
\mathbf{a} a\end{array}$} & \multirow{2}{*}{\multicolumn{2}{|c|}{$\frac{\bar{x} \quad \sigma_{\mathrm{x}}}{\text { Marabel bc } a}$}} & \multirow{2}{*}{\multicolumn{2}{|c|}{$\frac{\bar{x} \quad \sigma_{\mathrm{x}}}{\text { Nicola } \mathbf{a b} a}$}} & \multirow{2}{*}{$\begin{array}{l}\bar{x} \\
\text { Renate }\end{array}$} & \multirow{2}{*}{$\frac{\sigma_{\mathrm{x}}}{\text { bc } a}$} \\
\hline & & & & & & & & & & & & & & & & & & & \\
\hline & & $<1,055$ & & & & & & & & & & & & & & & & & \\
\hline & & $1,056-1,065$ & $\mathrm{a}$ & & & & & 0,33 & 0,02 & 0,27 & 0,06 & 0,62 & 0,12 & 0,38 & 0,13 & 0,52 & 0,13 & 0,43 & 0,08 \\
\hline & \multirow[t]{4}{*}{ Ernte } & $1,066-1,075$ & $a b$ & & & 0,13 & 0,06 & 0,39 & 0,07 & 0,28 & 0,06 & 0,49 & 0,07 & 0,35 & 0,11 & 0,41 & 0,20 & 0,36 & 0,10 \\
\hline & & $1,076-1,085$ & $a b c$ & 0,24 & 0,06 & 0,06 & 0,05 & & & & & & & & & 0,43 & 0,04 & 0,36 & 0,08 \\
\hline & & $1,086-1,095$ & $\mathrm{c}$ & 0,31 & 0,07 & 0,19 & 0,04 & & & & & & & & & & & & \\
\hline & & $>1,095$ & & & & & & & & & & & & & & & & & \\
\hline & & & & Adrett & cd $b$ & Afra & с $a$ & Gala & $\mathrm{d} b$ & Granola & b $a$ & Lolita & c $b$ & Marab & $\mathbf{d} b$ & Nicol & c $b$ & Renate & a $a$ \\
\hline & & $<1,055$ & $\mathrm{c}$ & & & & & 0,16 & 0,03 & & & & & & & & & & \\
\hline & & $1,056-1,065$ & $a b$ & 0,32 & 0,02 & & & 0,24 & 0,04 & 0,19 & 0,07 & 0,28 & 0,04 & 0,19 & 0,03 & 0,29 & 0,05 & 0,24 & 0,04 \\
\hline \multirow[t]{11}{*}{$2006 \mathbf{A}$} & 5 Monate Lagerung B & $1,066-1,075$ & $a b$ & 0,25 & 0,07 & 0,28 & 0,03 & 0,21 & 0,08 & 0,35 & 0,07 & 0,37 & 0,05 & 0,24 & 0,04 & 0,21 & 0,05 & 0,65 & 0,11 \\
\hline & & $1,076-1,085$ & $a b$ & 0,17 & 0,02 & 0,26 & 0,08 & 0,16 & 0,02 & 0,59 & 0,05 & 0,25 & 0,08 & 0,15 & 0,02 & 0,17 & 0,09 & 0,33 & 0,11 \\
\hline & & $1,086-1,095$ & $\mathrm{a}$ & 0,23 & 0,05 & 0,36 & 0,05 & & & & & 0,26 & 0,11 & & & 0,36 & 0,02 & 0,82 & 0,23 \\
\hline & & $>1,095$ & $a b$ & 0,24 & 0,06 & 0,31 & 0,06 & & & & & & & & & & & & \\
\hline & & & & Adrett & a $a$ & Afra & b $a$ & Gala & b $a b$ & Granola & b $a$ & Lolita & b $b$ & Marab & b $a$ & Nicol & b $a b$ & Renate & b $a$ \\
\hline & & $<1,055$ & $\mathrm{a}$ & & & & & 0,23 & 0,08 & & & & & & & 0,33 & 0,05 & & \\
\hline & & $1,056-1,065$ & $\mathrm{a}$ & 0,71 & 0,14 & & & 0,25 & 0,08 & 0,36 & 0,07 & 0,29 & 0,14 & 0,34 & 0,09 & 0,38 & 0,05 & 0,32 & 0,07 \\
\hline & 8 Monate Lagerung A & $1,066-1,075$ & $\mathrm{a}$ & 0,78 & 0,15 & 0,37 & 0,04 & 0,32 & 0,08 & 0,32 & 0,03 & 0,25 & 0,06 & 0,40 & 0,03 & 0,34 & 0,04 & 0,32 & 0,03 \\
\hline & & $1,076-1,085$ & $\mathrm{a}$ & 0,59 & 0,28 & 0,36 & 0,04 & & & 0,32 & 0,12 & 0,37 & 0,14 & 0,38 & 0,11 & & & 0,32 & 0,04 \\
\hline & & $1,086-1,095$ & $\mathrm{a}$ & 0,26 & 0,04 & 0,32 & 0,02 & & & 0,32 & 0,04 & 0,36 & 0,11 & 0,39 & 0,05 & & & 0,29 & 0,10 \\
\hline & & $>1,095$ & $\mathrm{a}$ & & & 0,41 & 0,02 & & & & & & & & & & & 0,39 & 0,30 \\
\hline
\end{tabular}


Tabelle 10.24 Binnendruck (Turgor) [Mittelwert $(\bar{x})$ und Standartabweichung $\left(\sigma_{\mathrm{x}}\right)$ ] (MPa) in Sorten und Knollen verschiedener spezifischer Dichten $\left(\mathrm{kg} \mathrm{L}^{-1}\right)$ nach der Ernte 2007 und nach fünf und acht Monaten Lagerung

\begin{tabular}{|c|c|c|c|c|c|c|c|c|c|c|c|c|c|c|c|c|c|c|c|}
\hline Jahr & Stadium & \multicolumn{2}{|c|}{ spezifische Dichte } & \multirow{3}{*}{$\frac{\bar{x}}{\text { Adrett }}$} & \multirow{2}{*}{$\frac{\sigma_{\mathrm{x}}}{\mathrm{a} a}$} & \multirow{2}{*}{\multicolumn{2}{|c|}{$\begin{array}{l}\bar{x} \quad \sigma_{\mathrm{x}} \\
\text { Afra d } a b\end{array}$}} & \multirow{2}{*}{$\frac{\bar{x}}{\text { Gala }}$} & \multirow{2}{*}{$\frac{\sigma_{\mathrm{x}}}{\mathrm{d} b}$} & \multirow{2}{*}{$\frac{\bar{x}}{\text { Granola }}$} & \multirow{2}{*}{$\begin{array}{l}\sigma_{\mathrm{x}} \\
\mathbf{c d} a b\end{array}$} & \multirow{2}{*}{\multicolumn{2}{|c|}{$\frac{\bar{x} \quad \sigma_{\mathrm{x}}}{\text { Lolita } \mathbf{a b} a}$}} & \multirow{2}{*}{\multicolumn{2}{|c|}{$\begin{array}{l}\bar{x} \quad \sigma_{\mathrm{x}} \\
\text { Marabel ab } a\end{array}$}} & \multirow{2}{*}{\multicolumn{2}{|c|}{$\frac{\bar{x} \quad \sigma_{\mathrm{x}}}{\text { Nicola } \mathbf{d} a}$}} & \multirow{2}{*}{$\frac{\bar{x}}{\text { Renate }}$} & \multirow{2}{*}{$\frac{\sigma_{\mathrm{x}}}{\text { bcd } b}$} \\
\hline & & & & & & & & & & & & & & & & & & & \\
\hline & & $<1,055$ & $a b$ & & & & & 0,22 & 0,07 & & & & & 0,28 & 0,06 & & & & \\
\hline & & $1,056-1,065$ & $\mathrm{ab}$ & 0,36 & 0,04 & & & 0,20 & 0,05 & 0,22 & 0,02 & 0,26 & 0,04 & 0,28 & 0,03 & 0,15 & 0,06 & 0,22 & 0,05 \\
\hline & \multirow[t]{4}{*}{ Ernte } & $1,066-1,075$ & $\mathrm{a}$ & 0,33 & 0,04 & 0,25 & 0,01 & 0,21 & 0,04 & 0,25 & 0,05 & 0,31 & 0,05 & 0,30 & 0,04 & 0,26 & 0,03 & 0,25 & 0,03 \\
\hline & & $1,076-1,085$ & $\mathrm{ab}$ & 0,34 & 0,04 & 0,23 & 0,03 & 0,20 & 0,04 & 0,25 & 0,04 & 0,29 & 0,05 & 0,26 & 0,03 & 0,27 & 0,02 & 0,26 & 0,03 \\
\hline & & $1,086-1,095$ & $\mathrm{a}$ & 0,29 & 0,04 & 0,25 & 0,03 & & & & & & & & & & & & \\
\hline & & $>1,095$ & $\mathrm{~b}$ & 0,21 & 0,03 & & & & & & & & & & & & & & \\
\hline & & & & Adret & a $a$ & Afra & ab $a$ & Gala & b $a$ & Granola & ab $a$ & Lolita & ab $a$ & Maral & ab $a$ & Nicol & ab $a$ & Renate & b $a b$ \\
\hline & & $<1,055$ & $\mathrm{a}$ & & & & & 0,30 & 0,04 & & & & & & & & & & \\
\hline & & $1,056-1,065$ & $\mathrm{a}$ & & & & & 0,29 & 0,04 & 0,32 & 0,05 & 0,29 & 0,02 & 0,29 & 0,03 & 0,32 & 0,04 & 0,26 & 0,04 \\
\hline \multirow[t]{11}{*}{$2007 \mathbf{B}$} & 5 Monate Lagerung A & $1,066-1,075$ & $\mathrm{a}$ & 0,31 & 0,07 & 0,34 & 0,07 & 0,26 & 0,03 & 0,34 & 0,01 & 0,27 & 0,04 & 0,29 & 0,04 & 0,29 & 0,05 & 0,30 & 0,03 \\
\hline & & $1,076-1,085$ & $\mathrm{a}$ & 0,32 & 0,04 & 0,26 & 0,04 & 0,24 & 0,03 & 0,27 & 0,06 & 0,26 & 0,04 & 0,34 & 0,06 & 0,31 & 0,05 & 0,28 & 0,03 \\
\hline & & $1,086-1,095$ & $\mathrm{a}$ & 0,35 & 0,02 & 0,30 & 0,03 & & & 0,30 & 0,02 & 0,32 & 0,02 & & & 0,26 & 0,04 & 0,26 & 0,02 \\
\hline & & $>1,095$ & $\mathrm{a}$ & 0,31 & 0,02 & 0,29 & 0,03 & & & & & & & & & & & & \\
\hline & & & & Adret & $\operatorname{cd} b$ & Afra & $\mathbf{d} b$ & Gala & bc $a b$ & Granola & $\mathbf{c d} b$ & Lolita & $\mathbf{a b} a$ & Maral & $\mathbf{a b} a$ & Nicol & $\mathbf{c d} a$ & Renate & a $a$ \\
\hline & & $<1,055$ & $\mathrm{a}$ & & & & & 0,25 & 0,00 & 0,27 & 0,07 & & & & & & & & \\
\hline & & $1,056-1,065$ & $\mathrm{a}$ & 0,20 & 0,04 & & & 0,26 & 0,04 & 0,22 & 0,04 & 0,32 & 0,06 & 0,30 & 0,06 & 0,23 & 0,06 & 0,33 & 0,06 \\
\hline & 8 Monate Lagerung $\mathrm{B}$ & $1,066-1,075$ & $\mathrm{a}$ & 0,21 & 0,03 & 0,14 & 0,01 & 0,23 & 0,02 & 0,24 & 0,04 & 0,33 & 0,10 & 0,35 & 0,10 & 0,24 & 0,05 & 0,35 & 0,06 \\
\hline & & $1,076-1,085$ & $\mathrm{a}$ & 0,26 & 0,09 & 0,24 & 0,07 & 0,32 & 0,06 & 0,21 & 0,06 & 0,31 & 0,08 & 0,32 & 0,05 & 0,21 & 0,02 & 0,36 & 0,08 \\
\hline & & $1,086-1,095$ & $\mathrm{a}$ & 0,24 & 0,05 & 0,19 & 0,03 & & & & & & & & & 0,13 & 0,15 & & \\
\hline & & $>1,095$ & $\mathrm{a}$ & 0,17 & 0,06 & 0,20 & 0,08 & & & & & & & & & & & & \\
\hline
\end{tabular}


Tabelle 10.25 Binnendruck (Turgor) [Mittelwert $(\bar{x})$ und Standartabweichung $\left(\sigma_{\mathrm{x}}\right)$ ] $(\mathrm{MPa})$ in Kronen- und Nabelgewebe nach der Ernte 2006 und 2007 und nach fünf und acht Monaten Lagerung der Sorten, deren Knollen nicht nach spezifischen Dichten getrennt wurden

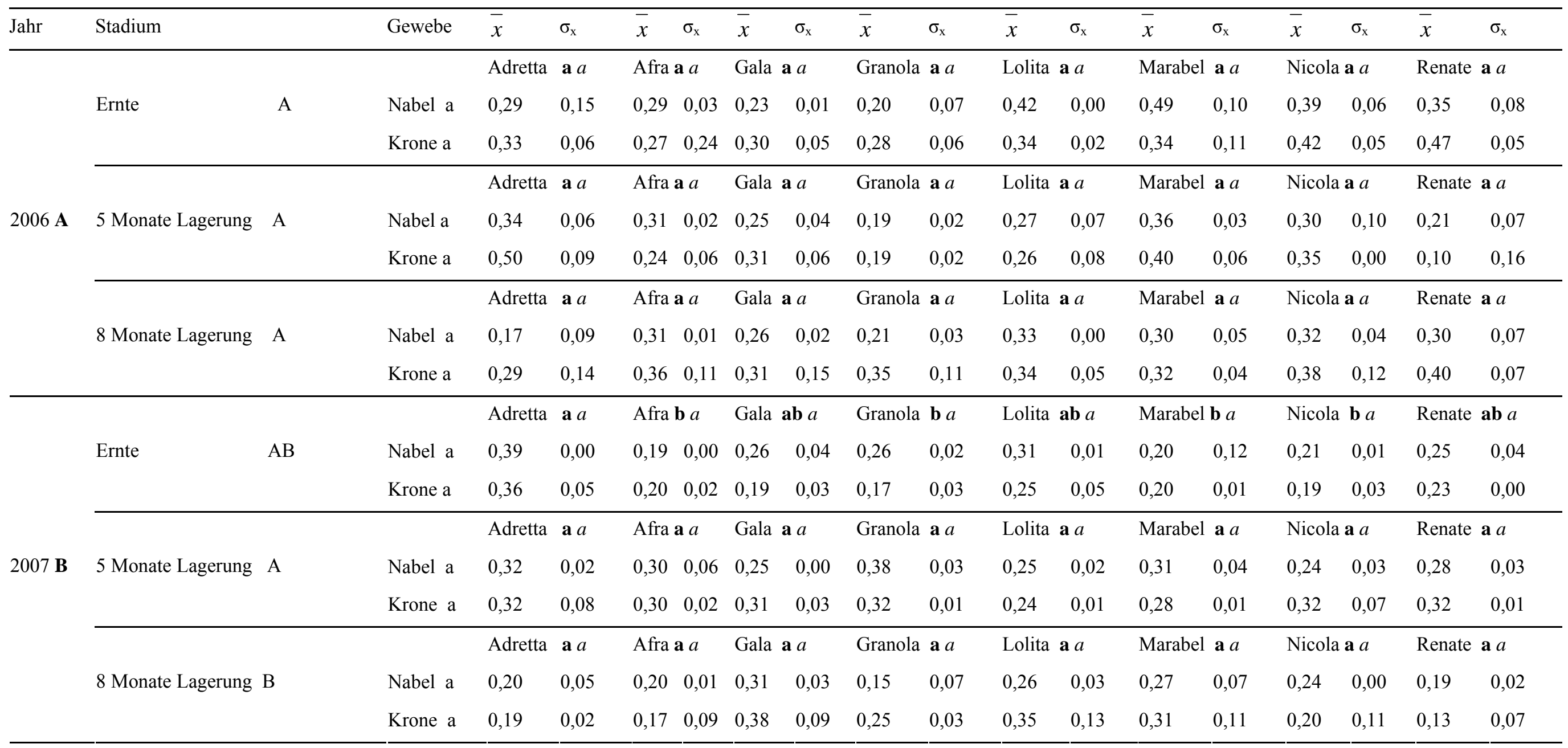


Tabelle 10.26 Volumetrischer Modulus der Elastizität [Mittelwert $(\bar{x})$ und Standartabweichung $\left(\sigma_{\mathrm{x}}\right)$ ] $(\mathrm{MPa})$ in Sorten und Knollen verschiedener spezifischer Dichten $\left(\mathrm{kg} \mathrm{L}^{-1}\right)$ nach der Ernte 2006 und nach fünf und acht Monaten Lagerung

\begin{tabular}{|c|c|c|c|c|c|c|c|c|c|c|c|c|c|c|c|c|c|c|c|c|}
\hline \multirow[t]{2}{*}{$\mathrm{Jahr}$} & \multicolumn{2}{|l|}{ Stadium } & \multicolumn{2}{|c|}{ spezifische Dichte } & \multirow{2}{*}{$\frac{\bar{x}}{\text { Adrett }}$} & & \multirow{2}{*}{$\begin{array}{l}\bar{x} \\
\text { Afra }\end{array}$} & \multirow{2}{*}{$\frac{\sigma_{\mathrm{x}}}{\mathbf{a} b}$} & \multirow{2}{*}{$\frac{\bar{x}}{\text { Gala }}$} & \multirow{2}{*}{$\frac{\sigma_{\mathrm{x}}}{\mathbf{a} a}$} & \multirow{2}{*}{$\frac{\bar{x}}{\text { Granola }}$} & & \multirow{2}{*}{$\frac{\bar{x}}{\text { Lolita }}$} & \multirow{2}{*}{$\frac{\sigma_{\mathrm{x}}}{\mathbf{a} a}$} & \multirow{2}{*}{\multicolumn{2}{|c|}{$\frac{\bar{x} \quad \sigma_{\mathrm{x}}}{\text { Marabel } \mathbf{a} b}$}} & \multirow{2}{*}{\multicolumn{2}{|c|}{$\frac{\bar{x} \quad \sigma_{\mathrm{x}}}{\text { Nicola } \mathbf{a} b}$}} & \multirow{3}{*}{$\begin{array}{l}\bar{x} \\
\text { Renate }\end{array}$} & \multirow{3}{*}{$\frac{\sigma_{\mathrm{x}}}{\mathbf{a} a}$} \\
\hline & & & & & & & & & & & & & & & & & & & & \\
\hline & & & $<1,055$ & & & & & & & & & & & & & & & & & \\
\hline & & & $1,056-1,065$ & $\mathrm{a}$ & & & & & 3,66 & 1,45 & 2,42 & 1,08 & 3,19 & 1,60 & 3,46 & 2,53 & 2,80 & 1,15 & 2,24 & 0,72 \\
\hline & \multirow[t]{4}{*}{ Ernte } & B & $1,066-1,075$ & $\mathrm{a}$ & & & 1,88 & 0,92 & 2,41 & 0,95 & 2,99 & 1,30 & 2,94 & 0,63 & 2,81 & 1,94 & 2,81 & 1,52 & 2,48 & 0,75 \\
\hline & & & $1,076-1,085$ & $\mathrm{a}$ & 2,33 & 0,71 & 1,46 & 0,53 & & & & & & & & & 2,64 & 0,69 & 2,40 & 1,72 \\
\hline & & & $1,086-1,095$ & $\mathrm{a}$ & 2,71 & 0,59 & 3,25 & 1,40 & & & & & & & & & & & & \\
\hline & & & $>1,095$ & & & & & & & & & & & & & & & & & \\
\hline & & & & & Adret & $\mathbf{a b} a$ & Afra & b $a b$ & Gala & b $a$ & Granola & ab $a$ & Lolita & ab $a$ & Marabel & ab $b$ & Nicola & $\mathbf{a b} b$ & Renate & a $a$ \\
\hline & & & $<1,055$ & $\mathrm{a}$ & & & & & 3,30 & 0,11 & & & & & & & & & & \\
\hline & & & $1,056-1,065$ & $\mathrm{a}$ & 3,52 & 1,29 & & & 4,38 & 2,09 & 2,16 & 1,38 & 6,96 & 1,57 & 4,24 & 1,49 & 3,92 & 1,95 & 4,41 & 1,76 \\
\hline \multirow[t]{11}{*}{$2006 \mathbf{A}$} & 5 Monate Lagerung & B & $1,066-1,075$ & $\mathrm{a}$ & 3,07 & 1,34 & 3,02 & 0,56 & 2,39 & 1,35 & 3,66 & 1,35 & 7,90 & 0,92 & 4,81 & 0,60 & 2,69 & 1,14 & 9,10 & 8,28 \\
\hline & & & $1,076-1,085$ & $\mathrm{a}$ & 3,45 & 0,53 & 2,83 & 0,23 & 2,16 & 0,68 & 5,27 & 4,82 & 4,95 & 1,69 & 3,02 & 0,54 & 2,94 & 1,50 & 5,20 & 3,18 \\
\hline & & & $1,086-1,095$ & $\mathrm{a}$ & 3,68 & 1,57 & 3,66 & 1,24 & & & & & 5,09 & 2,23 & & & 7,96 & 3,55 & 8,10 & 7,07 \\
\hline & & & $>1,095$ & $\mathrm{a}$ & 3,42 & 1,03 & 3,05 & 1,36 & & & & & & & & & & & & \\
\hline & & & & & Adret & b $a$ & Afra & $\mathbf{a b} a$ & Gala & $\mathbf{a b} a$ & Granola & ab $a$ & Lolita & $\mathbf{a b} a$ & Marabel & a $a$ & Nicol & a $a$ & Renate & a $a$ \\
\hline & & & $<1,055$ & $\mathrm{a}$ & & & & & 4,45 & 4,04 & & & & & & & 6,92 & 2,88 & 7,30 & 3,74 \\
\hline & & & $1,056-1,065$ & $\mathrm{a}$ & 2,67 & 1,08 & & & 4,30 & 2,64 & 6,00 & 3,05 & 6,35 & 3,07 & 6,83 & 2,71 & 8,38 & 4,37 & 10,57 & 12,69 \\
\hline & 8 Monate Lagerung & A & $1,066-1,075$ & $\mathrm{a}$ & 2,25 & 0,81 & 5,82 & 2,26 & 5,49 & 3,64 & 5,09 & 1,12 & 4,56 & 1,46 & 10,18 & 5,10 & 7,32 & 3,14 & 8,88 & 1,83 \\
\hline & & & $1,076-1,085$ & $\mathrm{a}$ & 2,64 & 0,48 & 4,96 & 1,90 & & & 5,08 & 2,48 & 7,35 & 2,64 & 9,65 & 2,14 & & & 6,13 & 3,32 \\
\hline & & & $1,086-1,095$ & $\mathrm{a}$ & 3,10 & 1,55 & 3,80 & 1,39 & & & 4,76 & 2,36 & 7,06 & 2,40 & 8,39 & 3,52 & & & 7,99 & 8,62 \\
\hline & & & $>1,095$ & $\mathrm{a}$ & & & 4,38 & 1,20 & & & & & & & & & & & & \\
\hline
\end{tabular}


Tabelle 10.27 Volumetrischer Modulus der Elastizität [Mittelwert $(\bar{x})$ und Standartabweichung $\left(\sigma_{\mathrm{x}}\right)$ ] $(\mathrm{MPa})$ in Sorten und Knollen verschiedener spezifischer Dichten $\left(\mathrm{kg} \mathrm{L}^{-1}\right)$ nach der Ernte 2007 und nach fünf und acht Monaten Lagerung

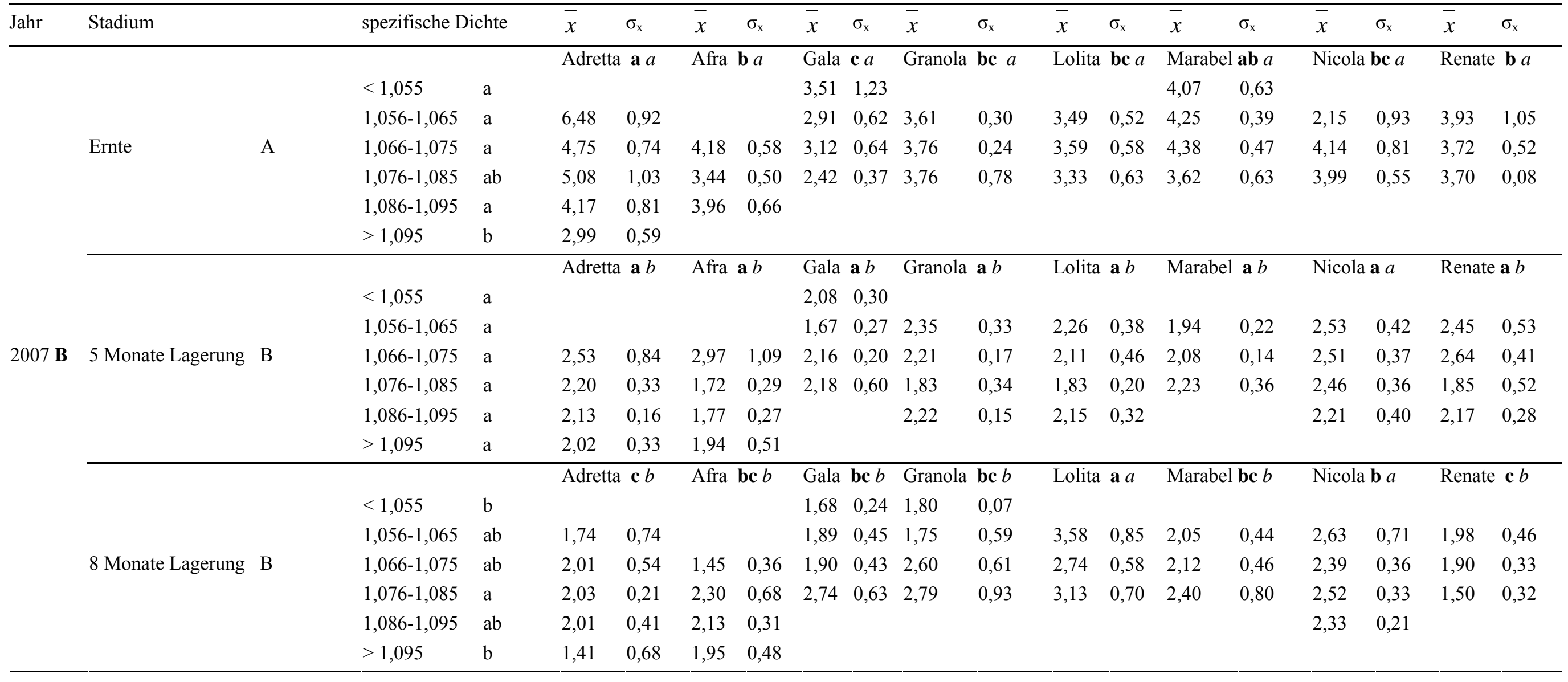


Tabelle 10.28 Volumetrischer Modulus der Elastizität [Mittelwert $(\bar{x})$ und Standartabweichung $\left(\sigma_{\mathrm{x}}\right)$ ] $(\mathrm{MPa})$ in Kronen- und Nabelgewebe nach der Ernte 2006 und 2007 und nach fünf und acht Monaten Lagerung der Sorten, deren Knollen nicht nach spezifischen Dichten getrennt wurden

\begin{tabular}{|c|c|c|c|c|c|c|c|c|c|c|c|c|c|c|c|c|c|c|c|}
\hline Jahr & Stadium & & Gewebe & $\bar{x}$ & $\sigma_{\mathrm{x}}$ & $\bar{x}$ & $\sigma_{\mathrm{x}}$ & $\bar{x}$ & $\sigma_{\mathrm{x}}$ & $\bar{x}$ & $\sigma_{\mathrm{x}}$ & $\bar{x}$ & $\sigma_{\mathrm{x}}$ & $\bar{x}$ & $\sigma_{\mathrm{x}}$ & $\bar{x}$ & $\sigma_{\mathrm{x}}$ & $\bar{x}$ & $\sigma_{\mathrm{x}}$ \\
\hline \multirow{9}{*}{2006 A } & \multirow{3}{*}{ Ernte } & \multirow{3}{*}{$\mathrm{B}$} & & \multicolumn{2}{|c|}{ Adretta a $a$} & \multicolumn{2}{|c|}{ Afra a $a$} & Gala & a $a$ & \multicolumn{2}{|c|}{ Granola a $a$} & \multicolumn{2}{|c|}{ Lolita a $a$} & \multicolumn{2}{|c|}{ Marabel a $a$} & Nicola & a $a$ & \multicolumn{2}{|c|}{ Renate a $a$} \\
\hline & & & Nabel a & 6,17 & 2,72 & 1,86 & 0,25 & 1,81 & 1,51 & 1,96 & 1,03 & 5,20 & 0,05 & 2,83 & 1,46 & 2,13 & 0,41 & 2,87 & 0,77 \\
\hline & & & Krone a & 5,75 & 4,20 & 2,09 & 1,53 & 2,11 & 2,03 & 3,30 & 1,10 & 3,00 & 1,89 & 3,06 & 0,96 & 2,27 & 0,78 & 2,73 & 1,31 \\
\hline & & & & \multicolumn{2}{|c|}{ Adretta a $a$} & \multicolumn{2}{|c|}{ Afra a $a$} & \multicolumn{2}{|c|}{ Gala a } & \multicolumn{2}{|c|}{ Granola a $a$} & \multicolumn{2}{|c|}{ Lolita a $a$} & \multicolumn{2}{|c|}{ Marabel a $a$} & \multicolumn{2}{|c|}{ Nicola a $a$} & \multicolumn{2}{|c|}{ Renate a $a$} \\
\hline & 5 Monate Lagerung & $\mathrm{AB}$ & Nabel a & 4,53 & 0,35 & 2,79 & 1,18 & 3,47 & 1,78 & 2,65 & 0,99 & 7,07 & 1,83 & 5,81 & 4,93 & 2,77 & 1,30 & 6,21 & 1,16 \\
\hline & & & Krone a & 7,53 & 1,80 & 2,75 & 1,11 & 5,13 & 1,25 & 3,16 & 0,38 & 6,07 & 1,53 & 6,78 & 5,44 & 4,66 & 0,20 & 2,57 & 2,23 \\
\hline & & & & \multicolumn{2}{|c|}{ Adretta a $a$} & \multicolumn{2}{|c|}{ Afra a $a$} & \multicolumn{2}{|c|}{ Gala a $a$} & \multicolumn{2}{|c|}{ Granola a $a$} & \multicolumn{2}{|c|}{ Lolita a $a$} & \multicolumn{2}{|c|}{ Marabel a $a$} & \multicolumn{2}{|c|}{ Nicola a $a$} & \multicolumn{2}{|c|}{ Renate a $a$} \\
\hline & \multirow[t]{2}{*}{8 Monate Lagerung } & A & Nabel a & 2,57 & 1,31 & 6,33 & 0,47 & 5,55 & 1,30 & 3,68 & 0,69 & 15,22 & 10,72 & 4,08 & 3,96 & 6,57 & 3,378 & 5,81 & 3,99 \\
\hline & & & Krone a & 4,14 & 1,72 & 3,94 & 1,68 & 4,39 & 5,33 & 3,32 & 2,95 & 10,40 & 3,06 & 6,18 & 0,73 & 10,87 & 3,16 & 9,37 & 1,99 \\
\hline & & & & Adretta & a $a$ & Afra & b $a$ & Gala & b $a$ & Granola & ab $a$ & Lolita & b $a$ & Mara & el b $a$ & Nicola & b $a$ & Renat & b $a$ \\
\hline & Ernte & A & Nabel a & 5,54 & 0,26 & 3,16 & 0,05 & 3,91 & 0,19 & 5,06 & 0,55 & 4,35 & 0,06 & 2,71 & 1,54 & 3,14 & 0,09 & 3,45 & 0,04 \\
\hline & & & Krone a & 6,18 & 1,15 & 3,31 & 0,45 & 2,47 & 0,44 & 3,01 & 0,56 & 3,59 & 0,76 & 2,74 & 0,06 & 2,47 & 0,39 & 2,99 & 0,12 \\
\hline & & & & Adretta & a $a b$ & Afra & & Gala & a $a$ & Granola & a $a b$ & Lolita & & Mara & el a $a$ & Nicola & a $a$ & Renat & a $a$ \\
\hline 2007 B & 5 Monate Lagerung & A & Nabel a & 2,66 & 0,52 & 1,91 & 0,89 & 1,92 & 0,13 & 2,31 & 0,13 & 2,28 & 0,42 & 2,74 & 0,965 & 2,23 & 0,42 & 2,27 & 0,17 \\
\hline & & & Krone a & 2,29 & 0,05 & 1,77 & 0,29 & 1,99 & 0,03 & 2,40 & 0,38 & 2,23 & 0,12 & 1,76 & 0,28 & 2,34 & 0,12 & 2,45 & 0,56 \\
\hline & & & & Adretta & a $b$ & Afra & & Gala & a $a$ & Granola & a $b$ & Lolita & & Mara & el a $a$ & Nicola & a $a$ & Renat & a $a$ \\
\hline & 8 Monate Lagerung & A & Nabel a & 2,15 & 1,33 & 2,00 & 0,03 & 1,93 & 1,09 & 1,29 & 0,86 & 2,32 & 0,49 & 2,34 & 0,09 & 2,22 & 0,07 & 2,46 & 0,69 \\
\hline & & & Krone a & 1,54 & 0,37 & 1,82 & 0,34 & 1,69 & 0,69 & 1,34 & 0,31 & 2,38 & 0,56 & 1,36 & 0,38 & 1,16 & 0,17 & 2,13 & 0,33 \\
\hline
\end{tabular}


Tabelle 10.29 Borkonzentration [Einzelwerte] ( $\left.\mathrm{mg} \mathrm{kg}^{-1} \mathrm{FM}\right)$ in Sorten und Knollen verschiedener spezifischer Dichten ( $\left.\mathrm{kg} \mathrm{L}^{-1}\right)$ nach der Vorernte und Ernte 2006 und nach fünf und acht Monaten Lagerung

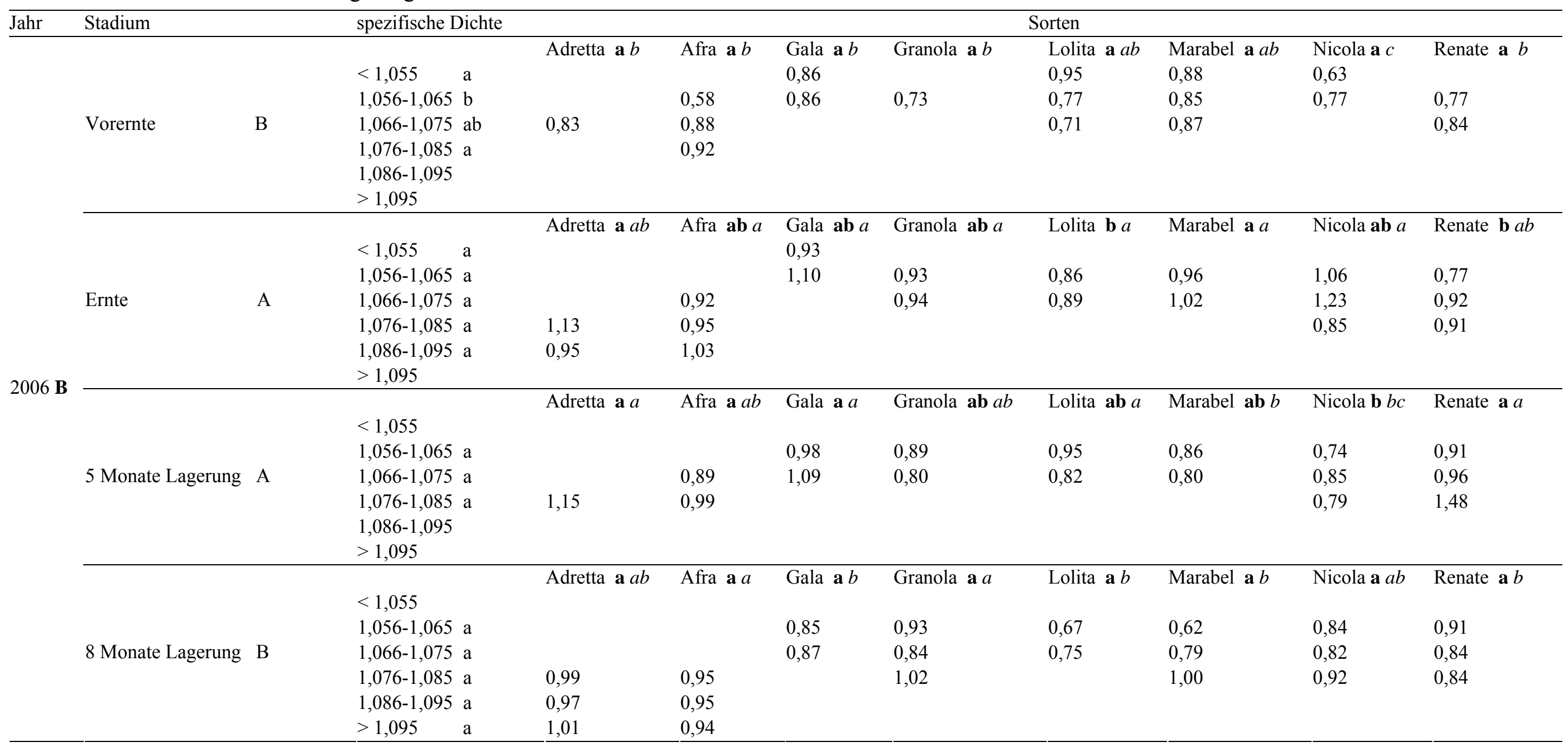


Tabelle 10.30 Borkonzentration [Einzelwerte] ( $\left.\mathrm{mg} \mathrm{kg}^{-1} \mathrm{FM}\right)$ in Sorten und Knollen verschiedener spezifischer Dichten (kg L $\left.{ }^{-1}\right)$ nach der Vorernte und Ernte 2007 und nach fünf und acht Monaten Lagerung

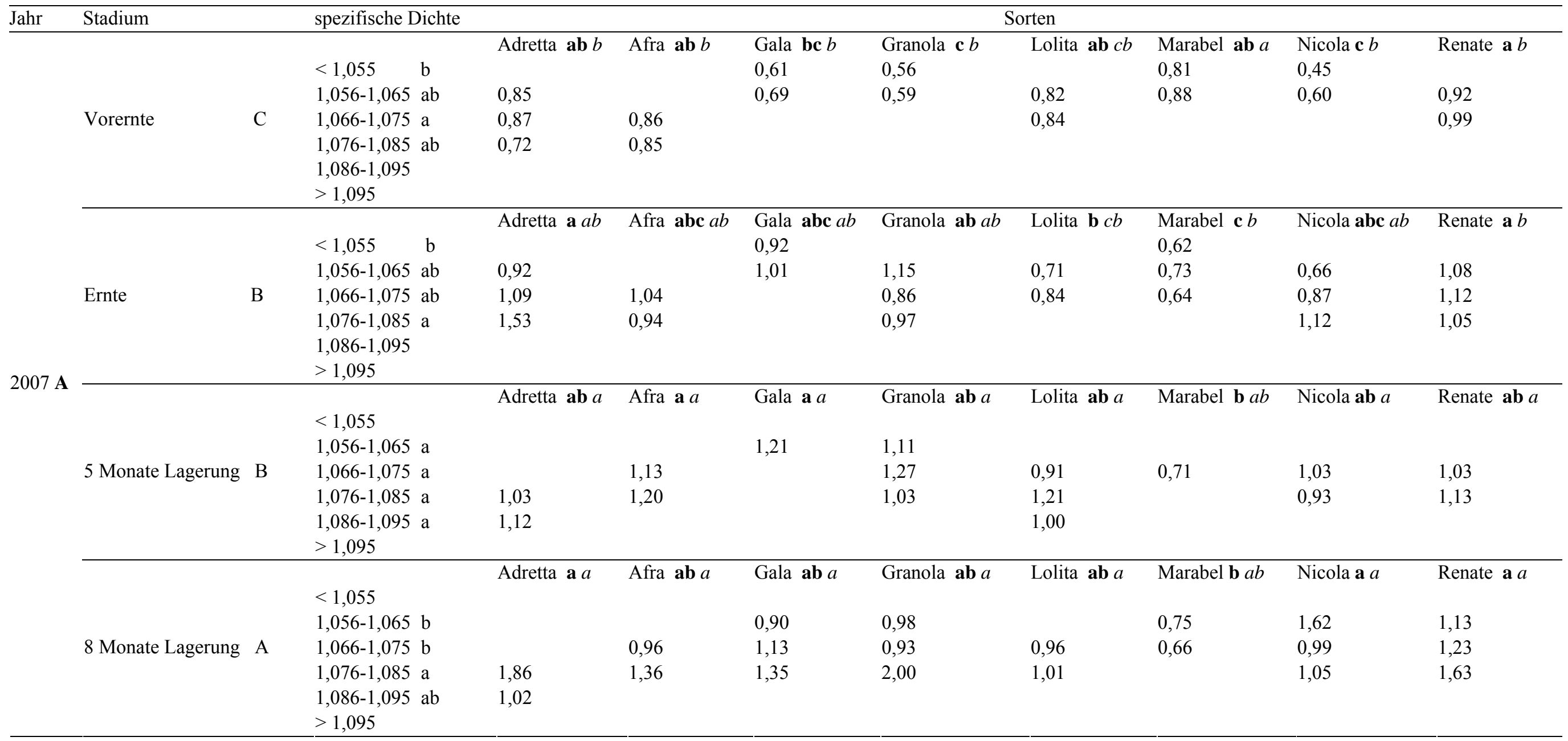


Tabelle 10.31 Eisenkonzentration [Einzelwerte] $\left(\mathrm{mg} \mathrm{kg}^{-1} \mathrm{FM}\right)$ in Sorten und Knollen verschiedener spezifischer Dichten $\left(\mathrm{kg} \mathrm{L}^{-1}\right)$ nach der Vorernte und Ernte 2006 und nach fünf und acht Monaten Lagerung

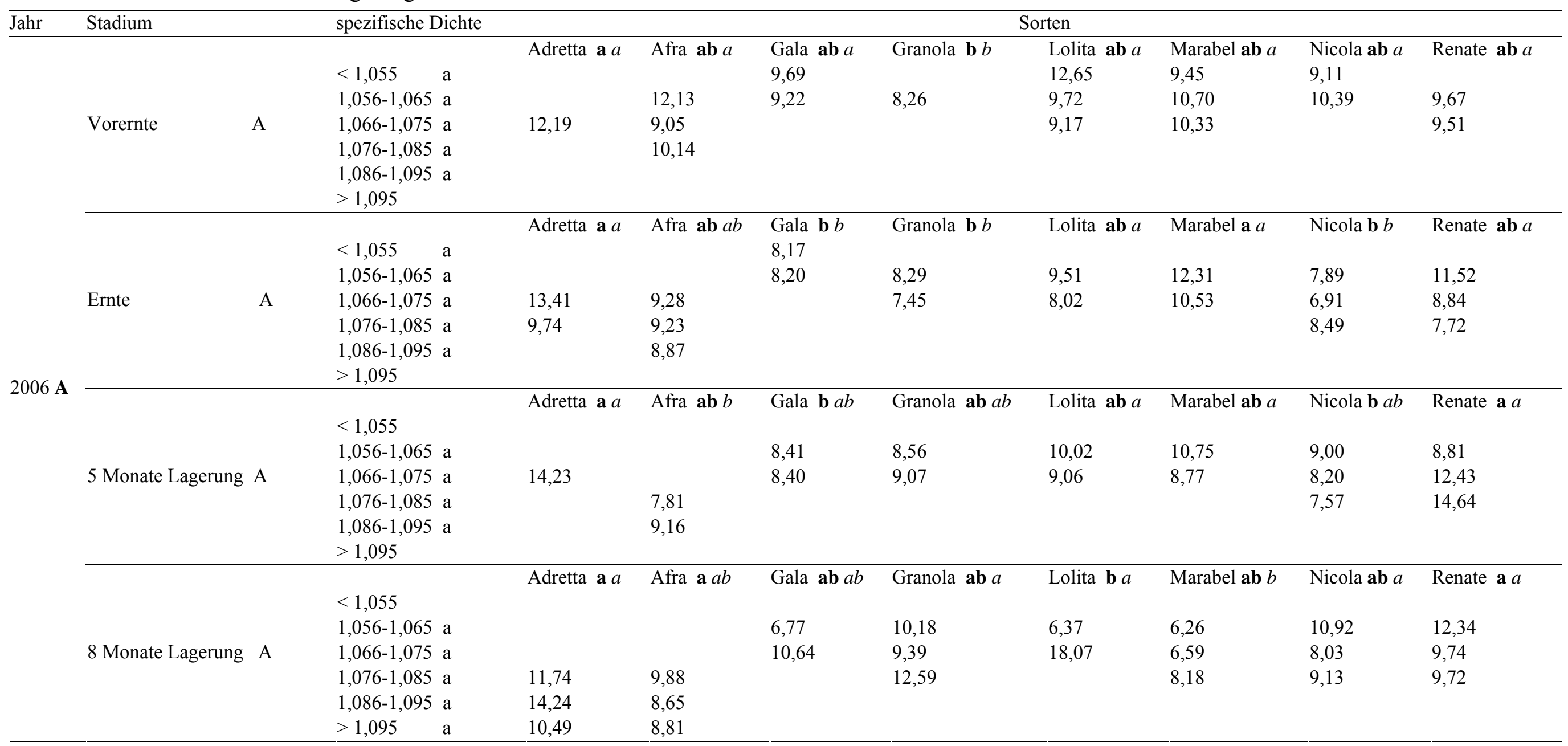


Tabelle 10.32 Eisenkonzentration [Einzelwerte] ( $\left.\mathrm{mg} \mathrm{kg}^{-1} \mathrm{FM}\right)$ in Sorten und Knollen verschiedener spezifischer Dichten ( $\left.\mathrm{kg} \mathrm{L}^{-1}\right)$ nach der Vorernte und Ernte 2007 und nach fünf und acht Monaten Lagerung

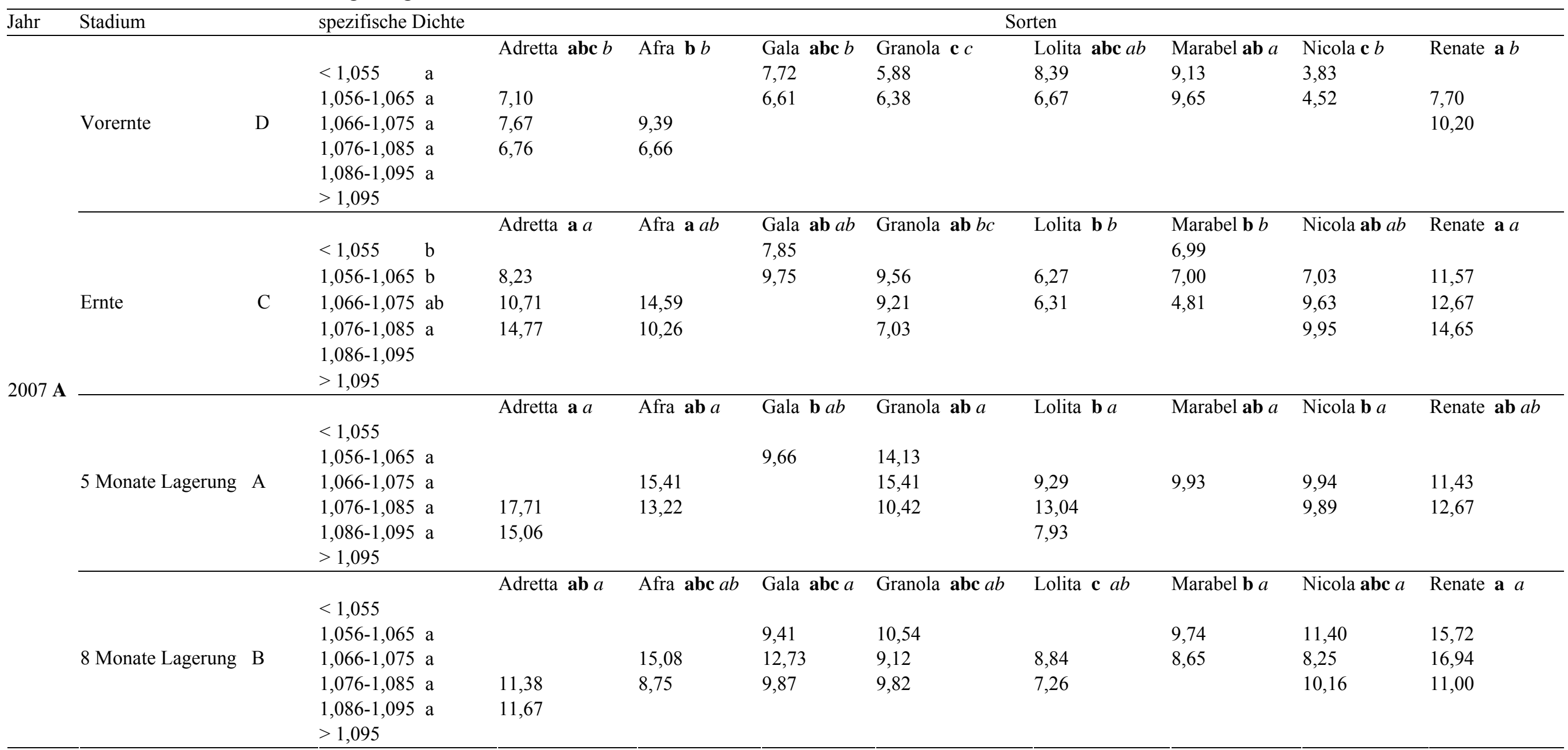


Tabelle 10.33 Selenkonzentration [Einzelwerte] $\left(\mathrm{mg} \mathrm{kg}^{-1} \mathrm{FM}\right)$ in Sorten und Knollen verschiedener spezifischer Dichten $\left(\mathrm{kg} \mathrm{L}^{-1}\right)$ nach der Vorernte und Ernte 2006 und nach fünf und acht Monaten Lagerung

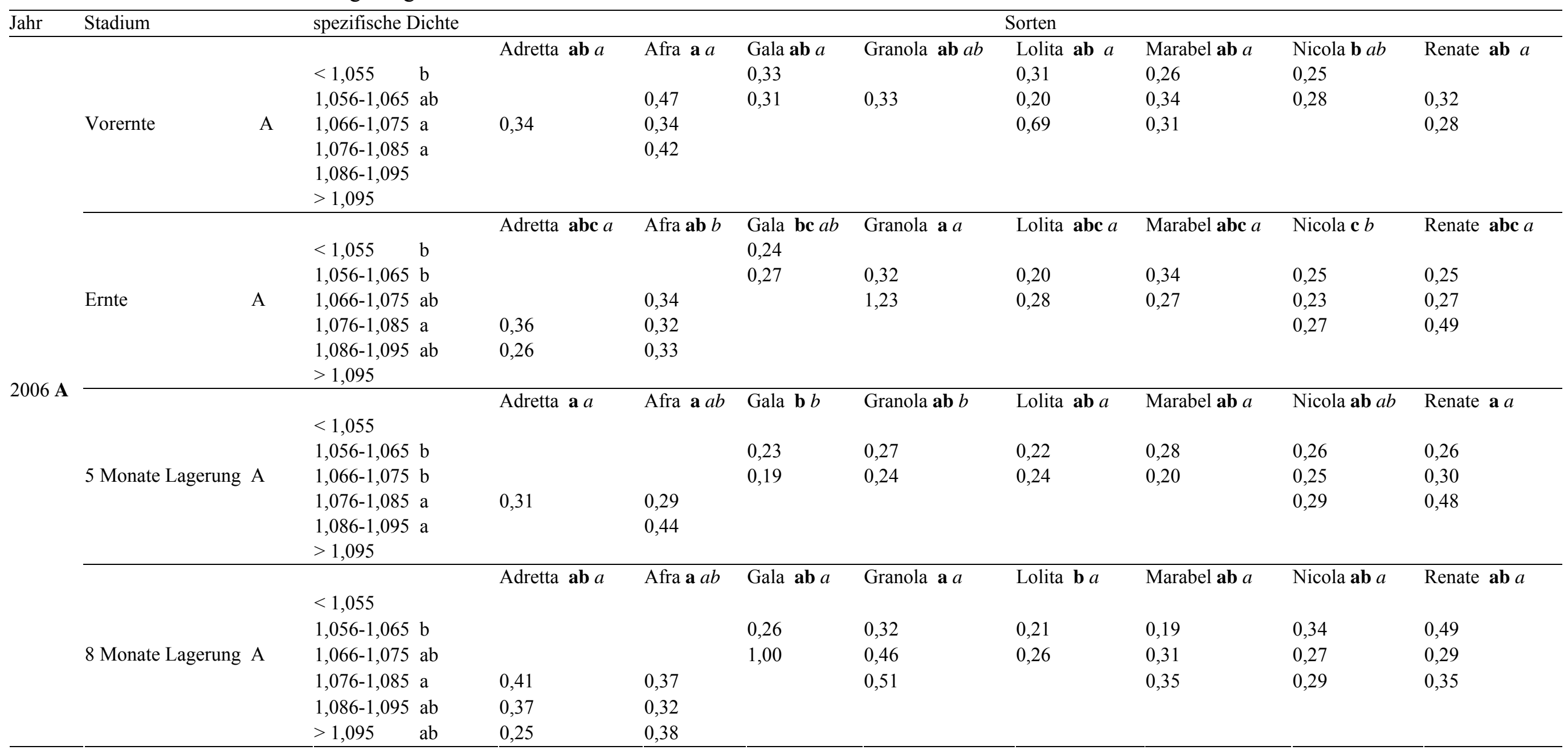


Tabelle 10.34 Selenkonzentration [Einzelwerte] ( $\left.\mathrm{mg} \mathrm{kg}^{-1} \mathrm{FM}\right)$ in Sorten und Knollen verschiedner spezifischer Dichten ( $\left.\mathrm{kg} \mathrm{L}^{-1}\right)$ nach der Vorernte und Ernte 2007 und nach fünf und acht Monaten Lagerung

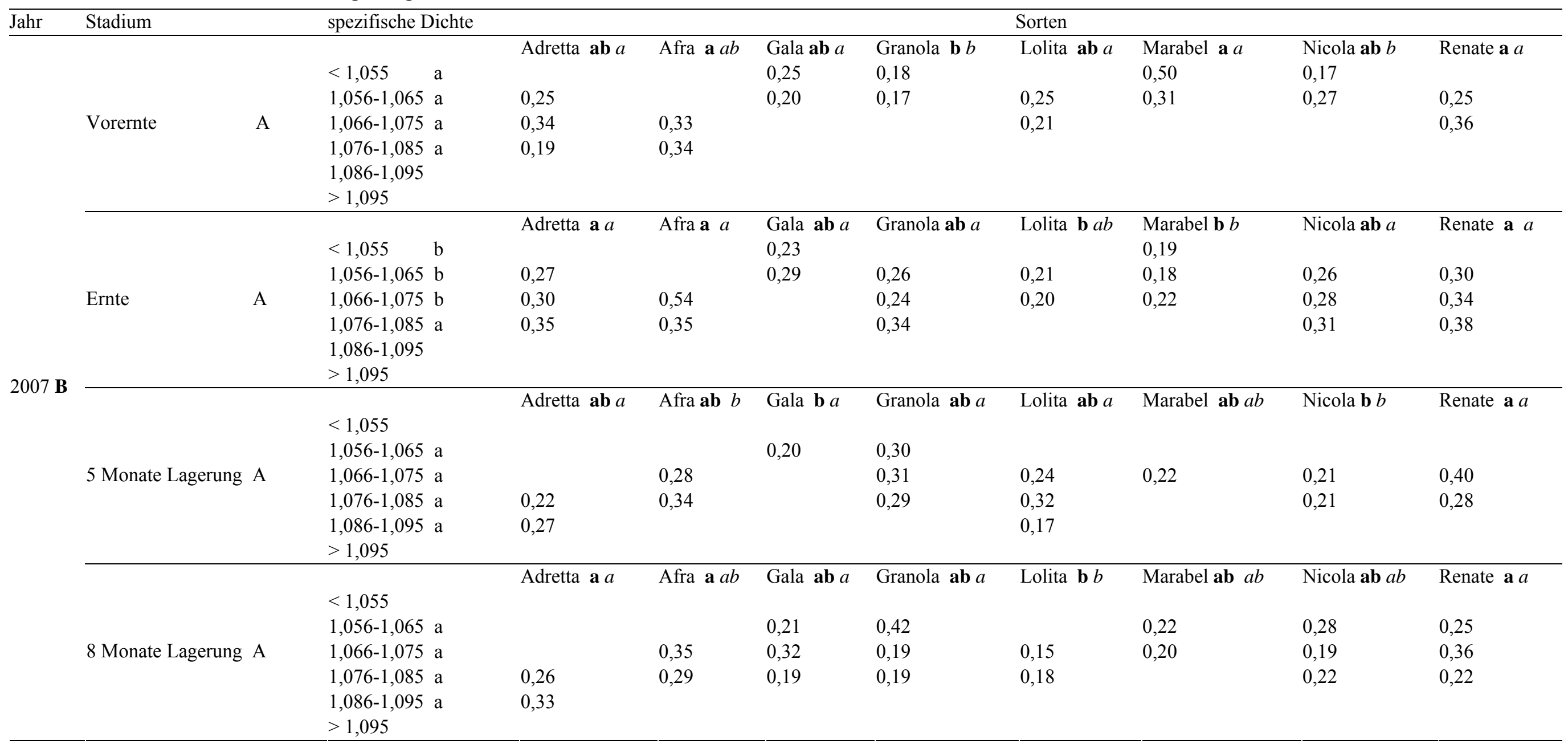


Tabelle 10.35 Kalziumkonzentration [Einzelwerte] $\left(\mathrm{mg} \mathrm{kg}^{-1} \mathrm{FM}\right)$ in Sorten und Knollen verschiedener spezifischer Dichten (kg L $\left.\mathrm{L}^{-1}\right)$ nach der Vorernte und Ernte 2006 und nach fünf und acht Monaten Lagerung

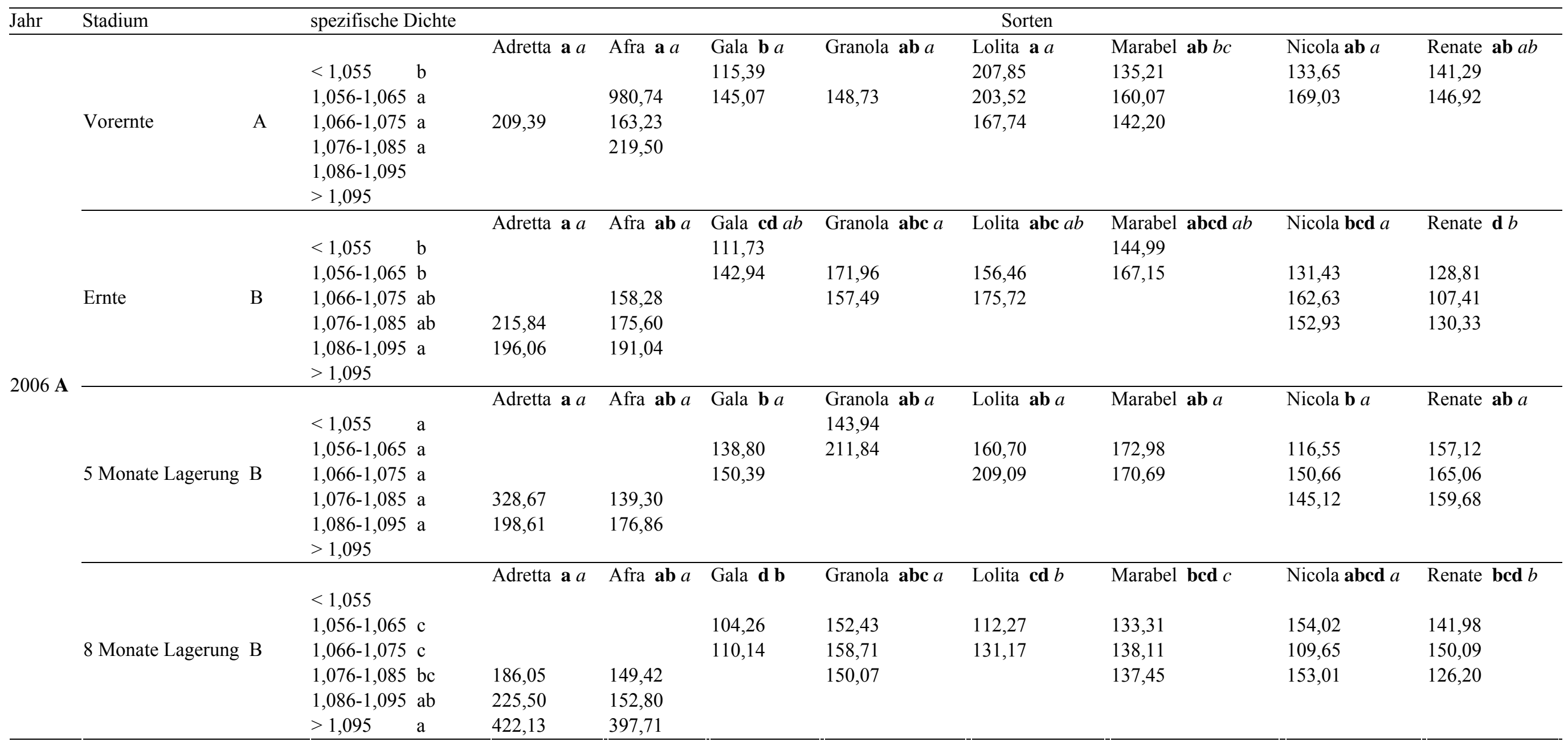


Tabelle 10.36 Kalziumkonzentration [Einzelwert] ( $\left.\mathrm{mg} \mathrm{kg}^{-1} \mathrm{FM}\right)$ in Sorten und Knollen verschiedener spezifischer Dichten (kg $\left.\mathrm{L}^{-1}\right)$ nach der Vorernte und Ernte 2007 und nach fünf und acht Monaten Lagerung

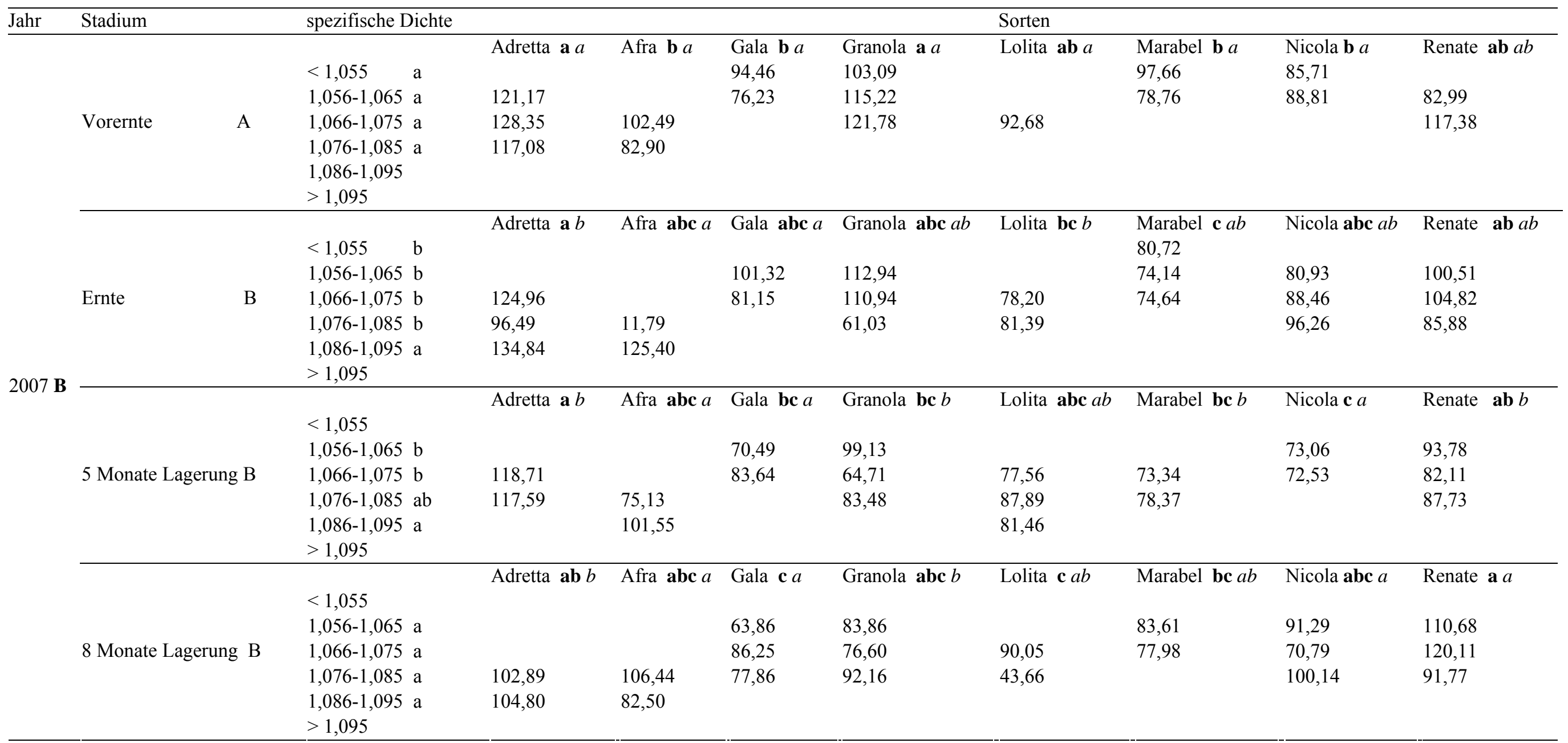


Tabelle 10.37 Magnesiumkonzentration [Einzelwerte] ( $\left.\mathrm{mg} \mathrm{kg}^{-1} \mathrm{FM}\right)$ in Sorten und Knollen verschiedener spezifischer Dichten (kg L $\left.{ }^{-1}\right)$ nach der Vorernte und Ernte 2006 und nach fünf und acht Monaten Lagerung

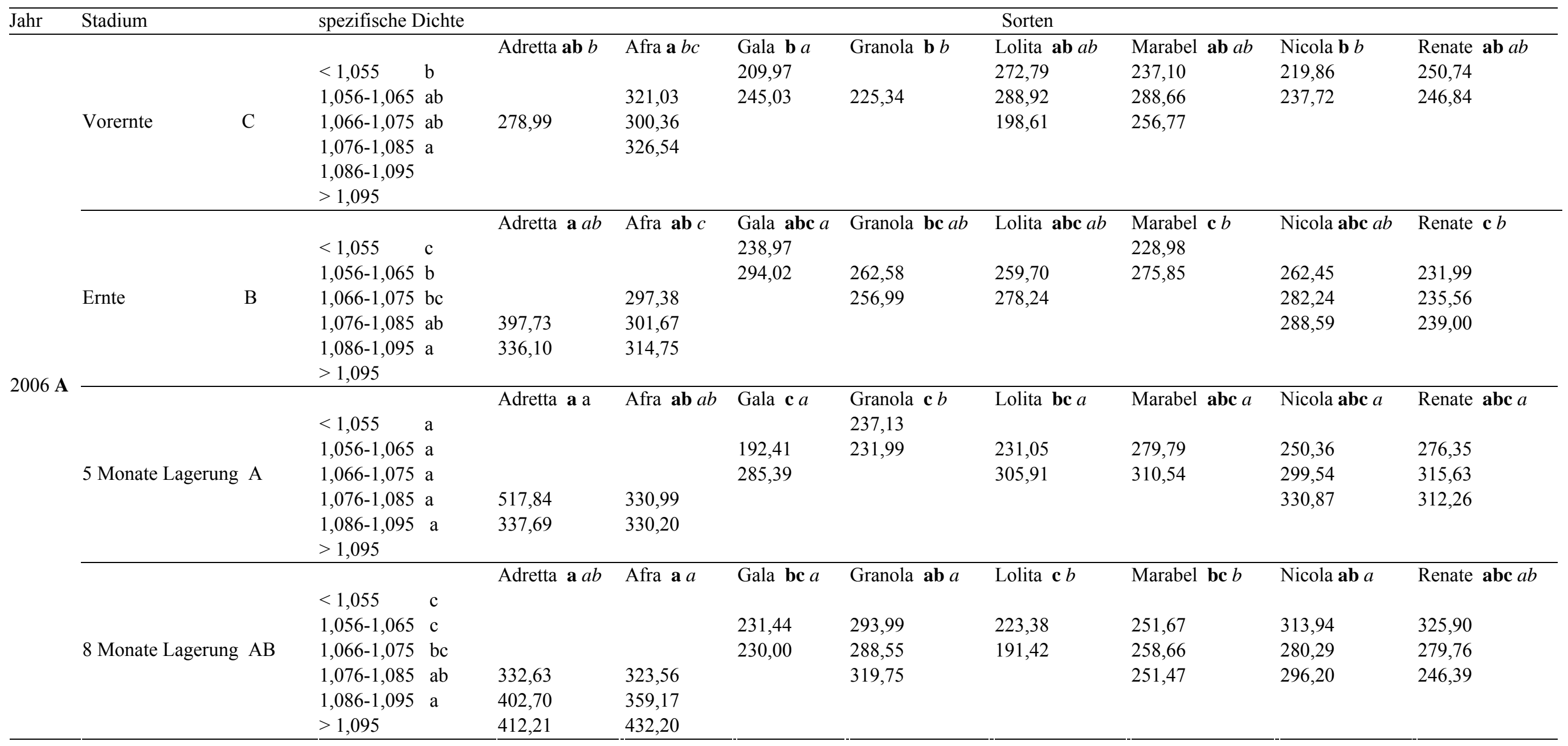


Tabelle 10.38 Magnesiumkonzentration [Einzelwerte] ( $\left.\mathrm{mg} \mathrm{kg}^{-1} \mathrm{FM}\right)$ in Sorten und Knollen verschiedener spezifischer Dichten (kg L $\left.{ }^{-1}\right)$ nach der Vorernte und Ernte 2007 und nach fünf und acht Monaten Lagerung

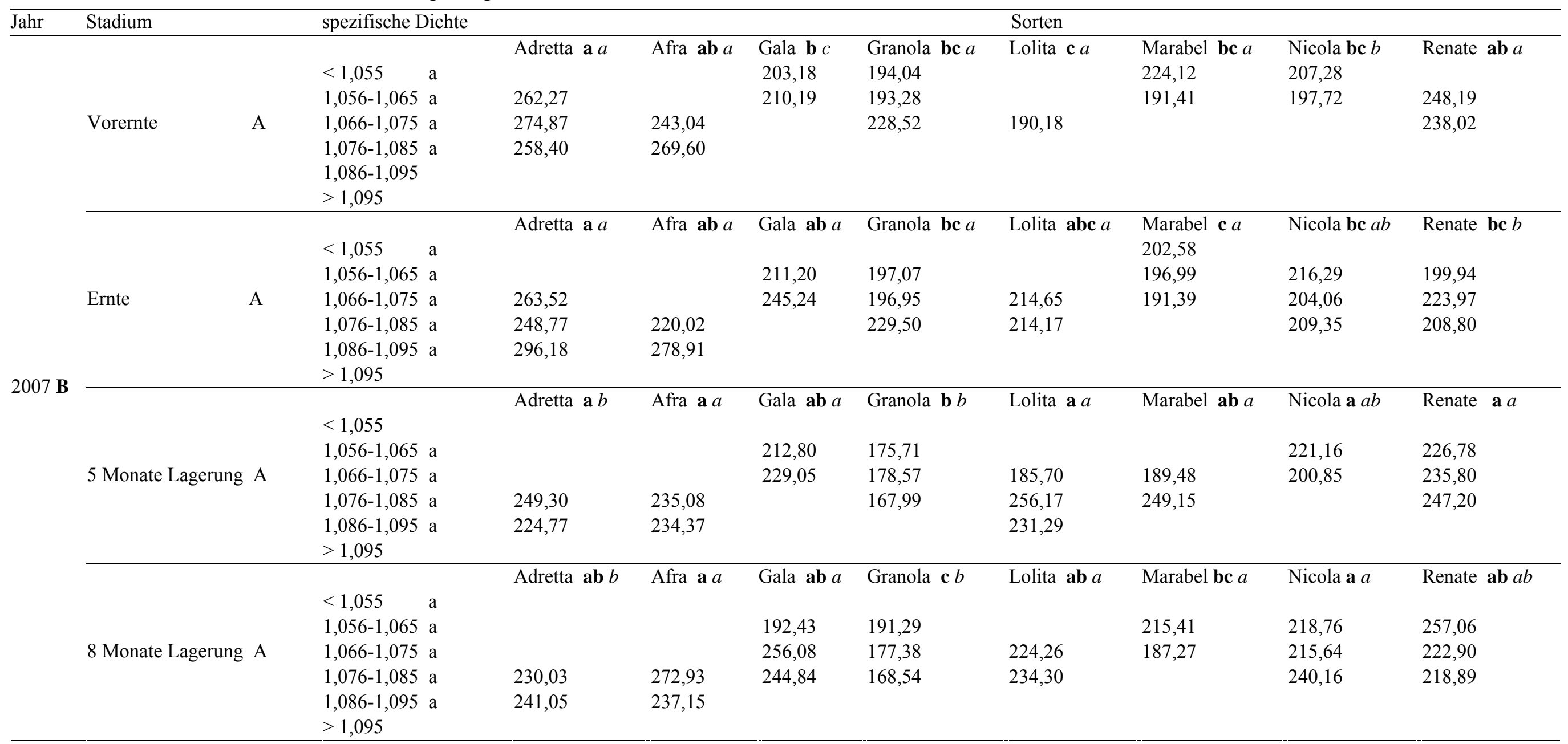


Tabelle 10.39 Kaliumkonzentration [Mittelwert $(\bar{x})$ und Standartabweichung $\left(\sigma_{\mathrm{x}}\right)$ ] $\left(\mathrm{mg} \mathrm{kg}^{-1} \mathrm{FM}\right)$ in Sorten und Knollen verschiedener spezifischer Dichten $\left(\mathrm{kg} \mathrm{L}^{-1}\right)$ nach der Vorernte und Ernte 2006 und nach fünf und acht Monaten Lagerung

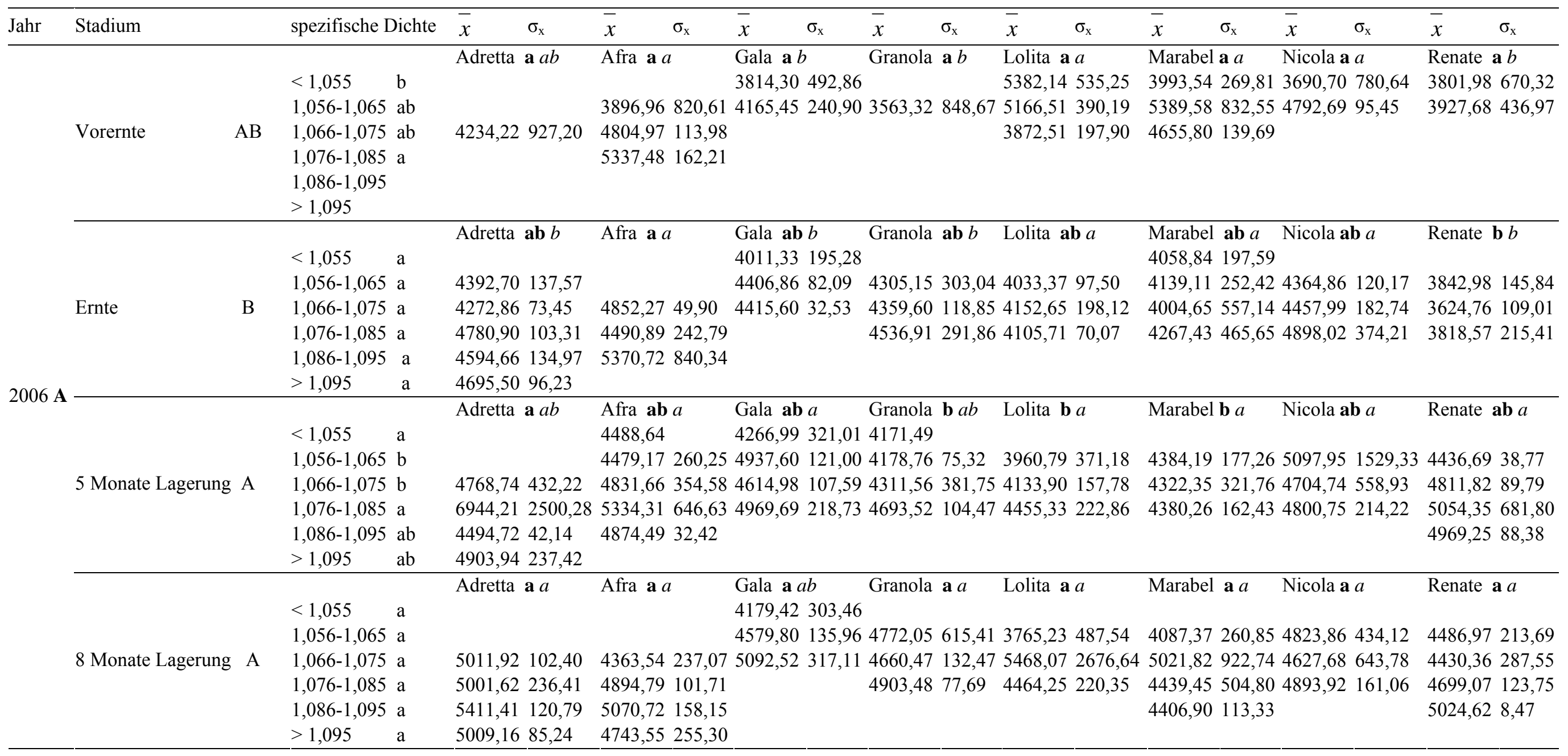


Tabelle 10.40 Kaliumkonzentration [Mittelwert $(\bar{x})$ und Standartabweichung $\left(\sigma_{\mathrm{x}}\right)$ ] $\left(\mathrm{mg} \mathrm{kg}^{-1} \mathrm{FM}\right)$ in Sorten und Knollen verschiedener spezifischer Dichten $\left(\mathrm{kg} \mathrm{L}^{-1}\right)$ nach der Vorernte und Ernte 2007 und nach fünf und acht Monaten Lagerung

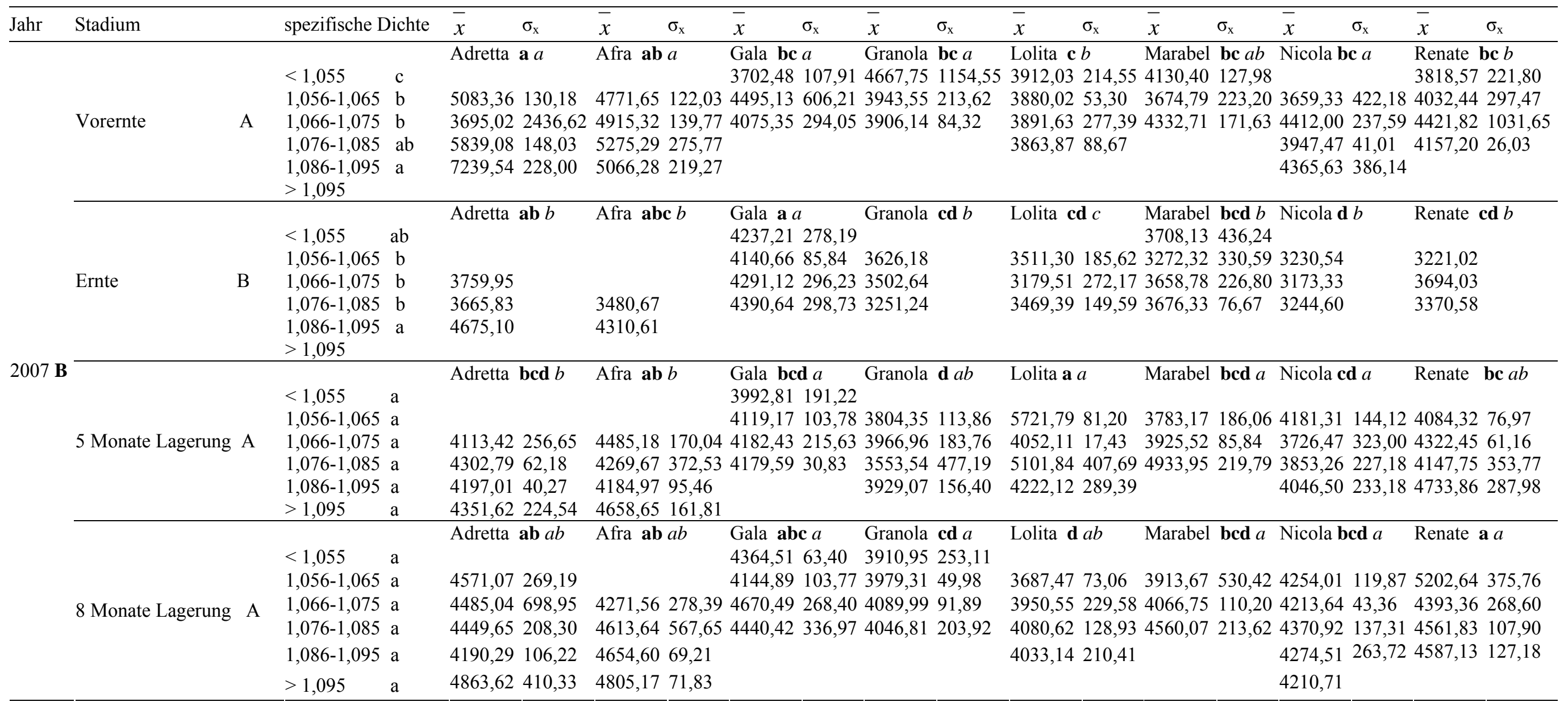


Tabelle 10.41 Phosphorkonzentration [Mittelwert $(\bar{x})$ und Standartabweichung $\left(\sigma_{\mathrm{x}}\right)$ ] $\left(\mathrm{mg} \mathrm{kg}^{-1} \mathrm{FM}\right)$ in Sorten und Knollen verschiedener spezifischer Dichten $\left(\mathrm{kg} \mathrm{L}^{-1}\right)$ nach der Ernte 2006 und nach fünf und acht Monaten Lagerung

\begin{tabular}{|c|c|c|c|c|c|c|c|c|c|c|c|c|c|c|c|c|c|c|c|c|}
\hline Jahr & Stadium & & spezifische D & ichte & $\overline{\bar{x}}$ & $\sigma_{\mathrm{x}}$ & $\overline{\bar{x}}$ & $\sigma_{\mathrm{x}}$ & $\bar{x}$ & $\sigma_{\mathrm{x}}$ & $\bar{x}$ & $\sigma_{\mathrm{x}}$ & $\overline{\bar{x}}$ & $\sigma_{\mathrm{x}}$ & $\overline{\bar{x}}$ & $\sigma_{\mathrm{x}}$ & $\bar{x}$ & $\sigma_{\mathrm{x}}$ & $\bar{x}$ & $\sigma_{\mathrm{x}}$ \\
\hline & & & & & Adretta & $\mathbf{a b} c$ & Afra $\mathbf{a b}$ & & Gala al & b $a$ & Granola & $\mathbf{a} b$ & Lolita & $\mathbf{a b} a$ & Marabel & b $a$ & Nicola a & $\mathbf{b} a b$ & Renate a & ab $b$ \\
\hline & & & $<1,055$ & $\mathrm{a}$ & & & & & 383,96 & 33,02 & & & & & 368,71 & 79,60 & & & & \\
\hline & & & $1,056-1,065$ & $\mathrm{a}$ & 345,28 & 53,75 & & & 409,10 & 28,22 & $457,45 ?$ & 35,27 & 376,22 & 37,19 & 306,57 & 46,81 & 417,76 & 31,66 & 414,88 & 37,84 \\
\hline & Ernte & B & $1,066-1,075$ & $\mathrm{a}$ & 384,29 & 11,06 & 347,15 & 17,60 & 384,23 & 11,62 & 469,47 & 7,56 & 315,56 & 34,59 & 288,31 & 18,11 & 420,88 & 12,98 & 380,46 & 41,84 \\
\hline & & & $1,076-1,085$ & $\mathrm{a}$ & 346,03 & 24,00 & 329,342 & 20,67 & & & 419,00 & 40,92 & 333,34 & 23,15 & 341,71 & 41,28 & 437,16 & 15,67 & 363,23 & 38,77 \\
\hline & & & $1,086-1,095$ & $\mathrm{a}$ & 343,16 & 30,24 & 389,815 & 58,19 & & & & & & & & & & & & \\
\hline & & & $>1,095$ & $\mathrm{a}$ & 346,44 & 7,89 & & & & & & & & & & & & & & \\
\hline & & & & & Adretta & abc $b$ & Afra $\mathbf{a b}$ & & Gala al & bc $a$ & Granola & a $a$ & Lolita & c $a$ & Marabel & bc $a$ & Nicola a & b $a$ & Renate & a $a$ \\
\hline & & & $<1,055$ & $\mathrm{a}$ & & & & & 432,86 & 8,88 & & & & & 394,49 & 7,10 & & & & \\
\hline & & & $1,056-1,065$ & $\mathrm{a}$ & & & & & 524,87 & 88,72 & 547,22 & 89,65 & 427,46 & 92,20 & 378,17 & 28,77 & 466,40 & 60,64 & 509,60 & 32,70 \\
\hline \multirow[t]{10}{*}{2006 A } & 5 Monate Lagerung & A & $1,066-1,075$ & $\mathrm{a}$ & 492,82 & 97,08 & 442,42 & 43,00 & 435,51 & 47,83 & 539,56 & 69,69 & 284,50 & 39,33 & 349,40 & 47,15 & 497,93 & 37,22 & 553,68 & 102,20 \\
\hline & & & $1,076-1,085$ & $\mathrm{a}$ & 535,11 & 142,74 & 470,64 & 16,09 & 443,85 & 40,08 & 569,94 & 109,04 & 353,64 & 24,80 & & & 480,10 & 21,99 & 478,27 & 57,38 \\
\hline & & & $1,086-1,095$ & $\mathrm{a}$ & 373,91 & 9,88 & 477,002 & 29,68 & & & & & & & & & & & 488,61 & 49,29 \\
\hline & & & $>1,095$ & $\mathrm{a}$ & 451,46 & 73,41 & 501,927 & 75,34 & & & & & & & & & & & & \\
\hline & & & $<1,055$ & $\mathrm{c}$ & Adretta & a $a$ & Afra $\mathbf{a b}$ & & $\begin{array}{l}\text { Gala c } \\
303,95\end{array}$ & $\begin{array}{l}a \\
40,81\end{array}$ & Granola & bc $b$ & Lolita & c $a$ & Marabel & c $a$ & Nicola & & Renate & c $b$ \\
\hline & & & $1,056-1,065$ & $\mathrm{c}$ & & & & & 313,09 & 44,65 & 389,65 & 197,50 & 408,80 & 45,29 & 411,03 & 42,39 & 349,23 & 26,71 & 263,04 & 38,17 \\
\hline & 8 Monate Lagerung & A & $1,066-1,075$ & $\mathrm{bc}$ & 580,09 & 74,56 & 288,76 & 17,66 & 541,36 & 74,07 & 353,07 & 38,96 & 366,50 & 24,94 & 301,58 & 9,24 & 309,50 & 54,44 & 335,81 & 32,82 \\
\hline & & & $1,076-1,085$ & $\mathrm{bc}$ & 544,02 & 75,59 & 607,445 & 58,02 & & & 535,90 & 50,49 & 395,85 & 54,19 & 340,69 & 53,37 & 399,53 & 48,76 & 282,95 & 23,36 \\
\hline & & & $1,086-1,095$ & $\mathrm{a}$ & 739,80 & 188,67 & 631,023 & 36,95 & & & & & & & 490,07 & 40,37 & & & 428,33 & 25,96 \\
\hline & & & $>1,095$ & $a b$ & 458,70 & 68,02 & 540,22 & 67,93 & & & & & & & & & & & & \\
\hline
\end{tabular}


Tabelle 10.42 Phosphorkonzentration [Mittelwert $(\bar{x})$ und Standartabweichung $\left(\sigma_{\mathrm{x}}\right)$ ] $\left(\mathrm{mg} \mathrm{kg}^{-1} \mathrm{FM}\right)$ in Sorten und Knollen verschiedener spezifischer Dichten $\left(\mathrm{kg} \mathrm{L}^{-1}\right)$ nach der Ernte 2007 und nach fünf und acht Monaten Lagerung

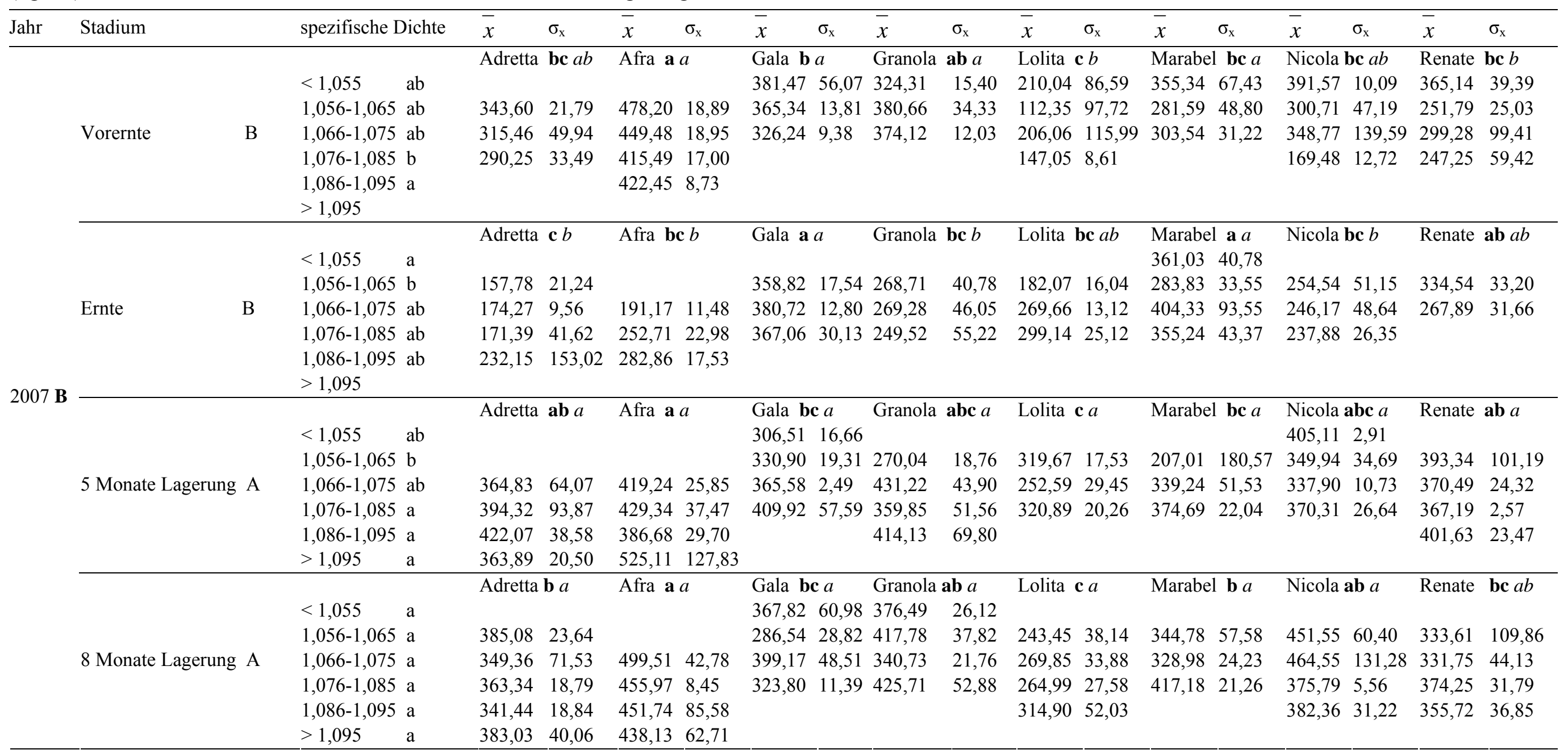


Tabelle 10.43 Anteil Knollen ( $\left.\mathrm{g} \mathrm{kg}^{-1} \mathrm{FM}\right)$ verschiedener spezifischer Dichten $\left(\mathrm{kg} \mathrm{L}^{-1}\right)$ innerhalb einer Sorte nach der Ernte 2005 und nach fünf und acht Monaten Lagerung

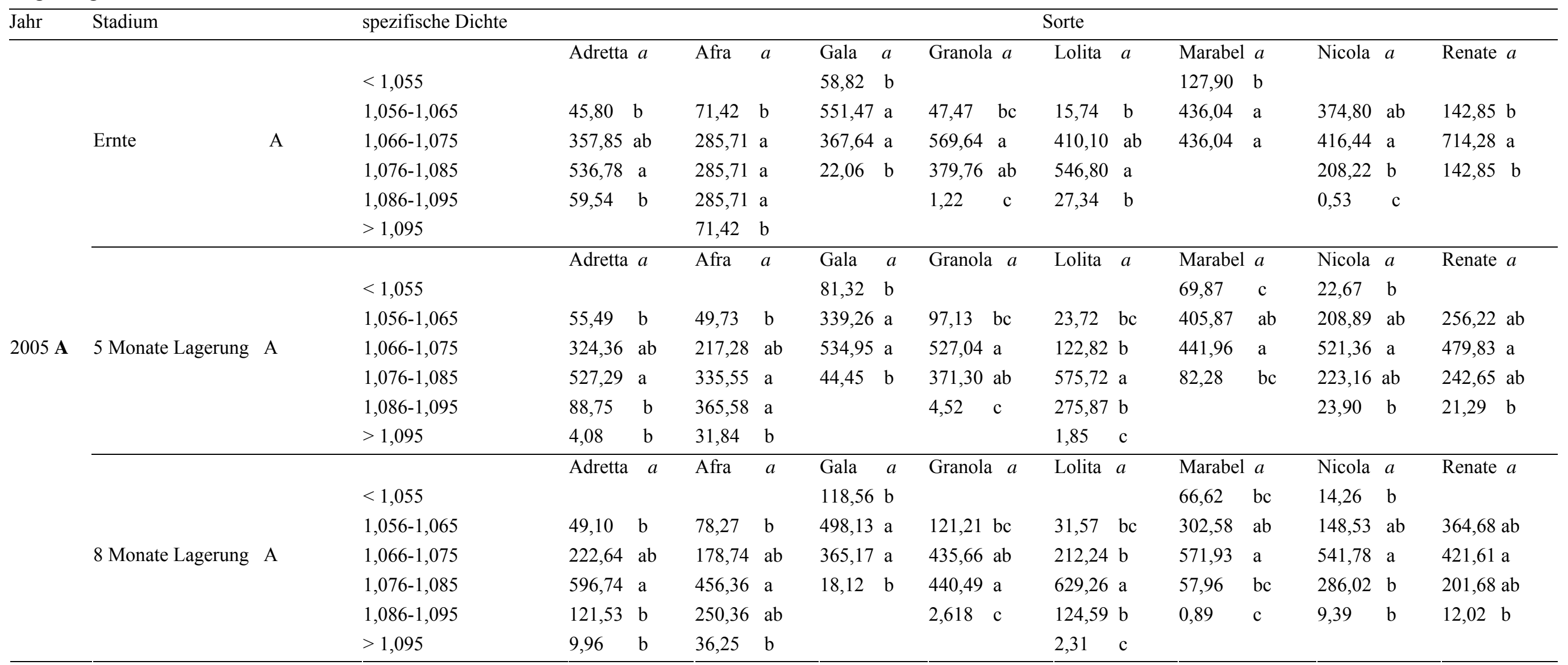


Tabelle 10.44 Anteil Knollen ( $\left.\mathrm{g} \mathrm{kg}^{-1} \mathrm{FM}\right)$ verschiedener spezifischer Dichten $\left(\mathrm{kg} \mathrm{L}^{-1}\right)$ innerhalb einer Sorte nach der Ernte 2006 und nach fünf und acht Monaten Lagerung

\begin{tabular}{|c|c|c|c|c|c|c|c|c|c|c|c|c|c|c|c|}
\hline Jahr & Stadium & & spezifische Dichte & & & & & & & & & & & & \\
\hline & & & & Adretta & $a$ & Afra & $a$ & Gala $a$ & Granola & $a$ & Lolita & $a$ & Marabel $a$ & Nicola $a$ & Renate $a$ \\
\hline & & & $<1,055$ & & & & & $94,06 \quad \mathrm{ab}$ & & & & & $41,706 \quad b$ & & \\
\hline & & & $1,056-1,065$ & 29,32 & $\mathrm{ab}$ & & & $411,44 \mathrm{ab}$ & 465,64 & $\mathrm{ab}$ & 383,53 & $a b$ & $244,94 a b$ & $332,73 \mathrm{ab}$ & $218,49 \mathrm{ab}$ \\
\hline & Ernte & A & $1,066-1,075$ & 88,05 & $\mathrm{ab}$ & 143,79 & $a b$ & 481,50 a & 502,20 & $\mathrm{a}$ & 578,30 & $a$ & 648,25 a & 501,71 a & $630,07 \mathrm{a}$ \\
\hline & & & $1,076-1,085$ & 546,95 & $\mathrm{a}$ & 451,15 & $\mathrm{a}$ & $12,97 \quad b$ & 32,14 & $\mathrm{~b}$ & 38,16 & $\mathrm{~b}$ & $65,09 \quad a b$ & $165,54 \mathrm{~b}$ & $144,76 \mathrm{ab}$ \\
\hline & & & $1,086-1,095$ & 319,29 & $\mathrm{ab}$ & 402,18 & $\mathrm{ab}$ & & & & & & & & 6,67 \\
\hline & & & $>1,095$ & 16,36 & $\mathrm{~b}$ & 2,86 & $\mathrm{~b}$ & & & & & & & & \\
\hline & & & & Adretta & $a$ & Afra & $a$ & Gala $a$ & Granola & $a$ & Lolita & $a$ & Marabel $a$ & Nicola $a$ & Renate $a$ \\
\hline & & & $<1,055$ & & & & & $53,53 \mathrm{ab}$ & & & & & & & \\
\hline & & & $1,056-1,065$ & 10,56 & $\mathrm{c}$ & & & $372,67 \mathrm{ab}$ & 330,20 & $\mathrm{ab}$ & 70,01 & $a b$ & $115,79 \mathrm{ab}$ & $129,63 \mathrm{ab}$ & $132,14 \quad a b$ \\
\hline 2006 A & 5 Monate Lagerung & A & $1,066-1,075$ & 69,22 & $\mathrm{bc}$ & 91,09 & $a b$ & $550,97 \mathrm{a}$ & 588,33 & $\mathrm{a}$ & 597,85 & $a$ & $612,51 \quad a$ & $545,21 \mathrm{a}$ & $509,40 \quad$ a \\
\hline & & & $1,076-1,085$ & 339,60 & $\mathrm{ab}$ & 394,92 & $\mathrm{ab}$ & $22,81 \quad b$ & 81,46 & $\mathrm{~b}$ & 319,08 & $\mathrm{ab}$ & $270,49 a b$ & $313,42 \mathrm{ab}$ & $335,21 \quad a b$ \\
\hline & & & $1,086-1,095$ & 538,44 & $\mathrm{a}$ & 493,62 & $\mathrm{a}$ & & & & 13,03 & $\mathrm{~b}$ & 1,19 & $11,73 \quad b$ & 23,24 \\
\hline & & & $>1,095$ & 42,16 & $\mathrm{bc}$ & 20,34 & $\mathrm{~b}$ & & & & & & & & \\
\hline & & & & Adretta & $a$ & Afra & $a$ & Gala $a$ & Granola & $a$ & Lolita & $a$ & Marabel $a$ & Nicola $a$ & Renate $a$ \\
\hline & & & $<1,055$ & & & & & $40,31 \mathrm{ab}$ & & & & & & & \\
\hline & & & $1,056-1,065$ & & & & & $333,40 \mathrm{ab}$ & 336,36 & $\mathrm{ab}$ & 160,35 & $\mathrm{ab}$ & $146,68 \mathrm{ab}$ & $189,59 \mathrm{ab}$ & $145,53 \mathrm{ab}$ \\
\hline & 8 Monate Lagerung & A & $1,066-1,075$ & 64,14 & $\mathrm{~b}$ & 50,56 & $\mathrm{~b}$ & 623,58 a & 479,43 & $\mathrm{a}$ & 545,73 & $\mathrm{a}$ & 515,89 a & 561,85 a & $369,04 \mathrm{ab}$ \\
\hline & & & $1,076-1,085$ & 223,47 & $\mathrm{ab}$ & 348,12 & $a b$ & $2,69 \quad b$ & 179,25 & $a b$ & 285,46 & $a b$ & $317,96 \mathrm{ab}$ & $245,35 \mathrm{ab}$ & 416,38 a \\
\hline & & & $1,086-1,095$ & 579,32 & $\mathrm{a}$ & 438,34 & $\mathrm{a}$ & & 4,94 & $\mathrm{~b}$ & 8,44 & $\mathrm{~b}$ & $19,45 \quad b$ & 3,20 & 63,36 \\
\hline & & & $>1,095$ & 133,05 & $a b$ & 162,96 & $a b$ & & & & & & & & 5,66 \\
\hline
\end{tabular}


Tabelle 10.45 Anteil Knollen ( $\left.\mathrm{g} \mathrm{kg}^{-1} \mathrm{FM}\right)$ verschiedener spezifischer Dichten $\left(\mathrm{kg} \mathrm{L}^{-1}\right)$ innerhalb einer Sorte nach der Ernte 2007 und nach fünf und acht Monaten Lagerung

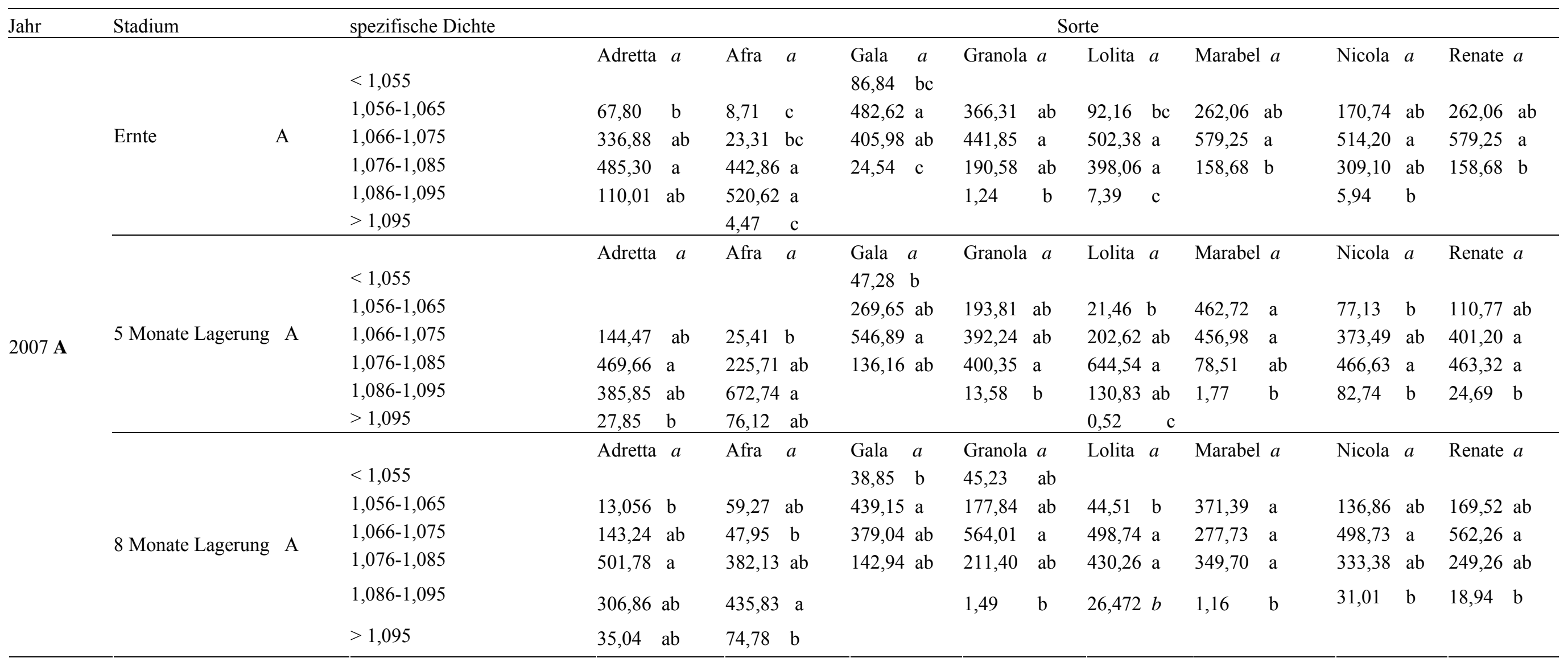


Tabelle 10.46 Trockenmassekonzentration [Mittelwert $(\bar{x})$ und Standartabweichung $\left(\sigma_{\mathrm{x}}\right)$ ] $\left(\mathrm{g} \mathrm{kg}^{-1} \mathrm{FM}\right)$ in Sorten und Knollen verschiedener spezifischer Dichten $\left(\mathrm{kg} \mathrm{L}^{-1}\right)$ nach der Ernte 2005 und nach fünf und acht Monaten Lagerung

\begin{tabular}{|c|c|c|c|c|c|c|c|c|c|c|c|c|c|c|c|c|c|c|c|}
\hline Jahr & \multicolumn{2}{|l|}{ Stadium } & spezifische Dichte & \multirow{2}{*}{$\begin{array}{l}\bar{x} \\
\text { Adretta }\end{array}$} & \multirow{2}{*}{$\begin{array}{l}\sigma_{\mathrm{x}} \\
\mathbf{a} a\end{array}$} & \multirow{3}{*}{$\begin{array}{l}\bar{x} \\
\text { Afra a } c\end{array}$} & $\sigma_{\mathrm{x}}$ & \multirow{2}{*}{\multicolumn{2}{|c|}{$\begin{array}{l}\bar{x} \quad \sigma_{\mathrm{x}} \\
\text { Gala a } a\end{array}$}} & \multirow{3}{*}{$\begin{array}{l}\bar{x} \\
\text { Granola }\end{array}$} & \multirow{3}{*}{$\begin{array}{c}\sigma_{\mathrm{x}} \\
\mathbf{a} a\end{array}$} & \multirow{3}{*}{$\frac{\bar{x}}{\text { Lolita }}$} & \multirow{3}{*}{$\frac{\sigma_{\mathrm{x}}}{\mathbf{a} a}$} & \multirow{2}{*}{\multicolumn{2}{|c|}{$\begin{array}{ll}\bar{x} & \sigma_{\mathrm{x}} \\
\text { Marabel a } a\end{array}$}} & \multirow{2}{*}{\multicolumn{2}{|c|}{$\begin{array}{ll}\bar{x} & \sigma_{\mathrm{x}} \\
\text { Nicola } a & a b\end{array}$}} & \multirow{3}{*}{$\begin{array}{l}\bar{x} \\
\text { Renate }\end{array}$} & \multirow{3}{*}{$\begin{array}{c}\sigma_{\mathrm{x}} \\
\mathbf{a} a\end{array}$} \\
\hline & & & & & & & & & & & & & & & & & & & \\
\hline & & & $<1,055$ & 161,82 & 0,62 & & & 138,80 & 0,29 & & & & & 141,93 & 1,15 & & & & \\
\hline & & & $1,056-1,065 \mathrm{~d}$ & 182,68 & 1,22 & 153,71 & 0,22 & 166,99 & 0,61 & 161,38 & 1,43 & 165,27 & 1,60 & 153,62 & 0,65 & 156,63 & 1,08 & 152,11 & 3,50 \\
\hline & \multirow[t]{7}{*}{ Ernte } & A & $1,066-1,075 \mathrm{c}$ & 186,01 & 29,12 & 178,79 & 2,69 & 178,97 & 0,29 & 184,55 & 1,44 & 187,21 & 1,02 & 180,63 & 1,14 & 183,67 & 1,07 & 178,63 & 4,24 \\
\hline & & & $1,076-1,085 \mathrm{~b}$ & 223,16 & 0,84 & 214,86 & 7,47 & 200,24 & 1,05 & 199,17 & 1,60 & 205,02 & 0,65 & & & 202,00 & 0,55 & 197,96 & 4,91 \\
\hline & & & $1,086-1,095$ a & & & 220,58 & 6,01 & & & & & 222,41 & 0,50 & & & & & & \\
\hline & & & $>1,095$ & & & 232,12 & 33,38 & & & & & & & & & & & & \\
\hline & & & & Adretta & a $a$ & Afra a $c$ & & Gala b $b$ & & Granola & ab $a$ & Lolita b & $b$ & Marabel & b $a$ & Nicola $\mathbf{b}$ & & Renate & ab $a$ \\
\hline & & & $<1,055$ & & & & & 130,98 & 14,80 & & & & & 138,08 & 1,75 & 138,73 & 3,31 & & \\
\hline & & & $1,056-1,065 \mathrm{~d}$ & 165,53 & 2,90 & 168,83 & 2,23 & 158,45 & 1,01 & 160,42 & 3,96 & 163,62 & 2,85 & 157,37 & 3,46 & 152,93 & 1,42 & 158,85 & 1,44 \\
\hline \multirow[t]{11}{*}{2005 C } & 5 Monate Lagerung & A & $1,066-1,075 \mathrm{c}$ & 178,04 & 9,82 & 186,91 & 3,28 & 160,71 & 21,75 & 194,55 & 17,45 & 178,53 & 5,04 & 176,01 & 1,23 & 167,32 & 2,46 & 179,66 & 3,05 \\
\hline & & & $1,076-1,085 \quad b$ & 230,17 & 21,70 & 206,03 & 2,10 & 201,25 & 6,70 & 193,50 & 4,11 & 180,33 & 25,26 & 189,81 & 1,31 & 192,13 & 4,61 & 203,07 & 3,71 \\
\hline & & & $1,0856-1,095 \mathrm{a}$ & 224,99 & 6,86 & 231,76 & 0,96 & & & & & 210,86 & 2,51 & & & 199,63 & 9,70 & 208,81 & 13,39 \\
\hline & & & $>1,095$ & & & 240,91 & 5,17 & & & & & & & & & & & & \\
\hline & & & & Adretta & ab $a$ & Afra $\mathbf{a} c$ & & Gala b c & & Granola & a $a$ & Lolita b & $a b$ & Marabel & c $b$ & Nicola a & b $a$ & Renate & b $a$ \\
\hline & & & $<1,055$ & & & & & 131,84 & 3,41 & & & & & 131,51 & 6,78 & 128,55 & 7,84 & & \\
\hline & & & $1,056-1,065 \mathrm{e}$ & 161,45 & 0,88 & 170,40 & 3,03 & 154,53 & 4,19 & 159,77 & 1,72 & 161,47 & 3,06 & 152,49 & 1,75 & 153,58 & 0,65 & 152,48 & 4,20 \\
\hline & 8 Monate Lagerung & A & $1,066-1,075 \mathrm{~d}$ & 185,68 & 1,04 & 188,86 & 2,32 & 181,25 & 1,77 & 180,69 & 5,26 & 176,03 & 3,15 & 168,89 & 0,65 & 195,34 & 0,74 & 182,51 & 3,23 \\
\hline & & & $1,076-1,085 \mathrm{c}$ & 200,77 & 0,68 & 207,97 & 4,40 & 198,91 & 4,17 & 199,42 & 2,98 & 203,42 & 3,13 & 190,60 & 0,92 & 203,38 & 5,65 & 199,31 & 4,51 \\
\hline & & & $1,086-1,095 \mathrm{~b}$ & 223,68 & 2,02 & 222,99 & 4,78 & & & & & 211,16 & 4,44 & & & 213,05 & 2,16 & 215,34 & 5,99 \\
\hline & & & $>1,095$ & 240,65 & 2,18 & 234,48 & 17,09 & & & & & & & & & & & & \\
\hline
\end{tabular}


Tabelle 10.47 Trockenmassekonzentration [Mittelwert $(\bar{x})$ und Standartabweichung $\left(\sigma_{\mathrm{x}}\right)$ ] $\left(\mathrm{g} \mathrm{kg}^{-1} \mathrm{FM}\right)$ in Sorten und Knollen verschiedener spezifischer Dichten $\left(\mathrm{kg} \mathrm{L}^{-1}\right)$ nach der Ernte 2006 und nach fünf und acht Monaten Lagerung

\begin{tabular}{|c|c|c|c|c|c|c|c|c|c|c|c|c|c|c|c|c|c|c|c|}
\hline \multirow[t]{10}{*}{ Jahr } & Stadium & \multicolumn{2}{|c|}{ spezifische Dichte } & \multirow{3}{*}{$\begin{array}{l}\bar{x} \\
\text { Adretta }\end{array}$} & \multirow{2}{*}{$\frac{\sigma_{\mathrm{x}}}{\mathrm{a} a}$} & \multirow{2}{*}{\multicolumn{2}{|c|}{$\begin{array}{l}\overline{\bar{x}} \quad \sigma_{\mathrm{x}} \\
\text { Afra } \mathbf{a b} a\end{array}$}} & \multirow{2}{*}{\multicolumn{2}{|c|}{$\begin{array}{l}\bar{x} \quad \sigma_{\mathrm{x}} \\
\text { Gala } \mathbf{d} a b\end{array}$}} & \multirow{2}{*}{\multicolumn{2}{|c|}{$\begin{array}{l}\overline{\bar{x}} \quad \sigma_{\mathrm{x}} \\
\text { Granola bcd } a\end{array}$}} & \multirow{2}{*}{$\begin{array}{l}\bar{x} \\
\text { Lolita }\end{array}$} & \multirow{2}{*}{$\frac{\sigma_{\mathrm{x}}}{\operatorname{bcd} a}$} & \multirow{2}{*}{\multicolumn{2}{|c|}{$\begin{array}{l}\overline{\bar{x}} \quad \sigma_{\mathrm{x}} \\
\text { Marabel bcd } a\end{array}$}} & \multirow{3}{*}{$\begin{array}{l}\bar{x} \\
\text { Nicola } \mathbf{c}\end{array}$} & \multirow{3}{*}{$\begin{array}{l}\sigma_{\mathrm{x}} \\
a\end{array}$} & \multirow{3}{*}{$\begin{array}{l}\bar{x} \\
\text { Renate }\end{array}$} & \multirow{3}{*}{$\begin{array}{c}\sigma_{\mathrm{x}} \\
\mathbf{e} b\end{array}$} \\
\hline & & & & & & & & & & & & & & & & & & & \\
\hline & & $<1,055$ & $\mathrm{e}$ & & & & & 141,35 & 2,43 & & & & & 159,43 & 2,38 & & & & \\
\hline & & $1,056-1,065$ & d & 188,31 & 0,66 & & & 166,36 & 2,96 & 169,68 & 2,52 & 174,09 & 2,89 & 170,85 & 3,88 & 168,13 & 1,58 & 149,22 & 3,63 \\
\hline & \multirow[t]{6}{*}{ Ernte } & $1,066-1,075$ & $\mathrm{c}$ & 193,46 & 4,66 & 193,02 & 2,16 & 184,92 & 4,61 & 190,46 & 4,56 & 192,78 & 3,52 & 186,72 & 3,38 & 183,00 & 1,97 & 159,03 & 7,93 \\
\hline & & $1,076-1,085$ & $\mathrm{~b}$ & 213,56 & 2,37 & 210,69 & 2,30 & 195,81 & 0,71 & 205,54 & 5,97 & 197,16 & 2,64 & 205,69 & 3,49 & 200,92 & 3,00 & 176,70 & 3,86 \\
\hline & & $1,086-1,095$ & $\mathrm{a}$ & 229,07 & 1,10 & 231,38 & 5,33 & & & & & & & & & & & 188,01 & 3,60 \\
\hline & & $>1,095$ & $\mathrm{a}$ & 244,50 & 3,84 & 224,30 & 14,94 & & & & & & & & & & & & \\
\hline & & $<1,055$ & $\mathrm{~b}$ & Adretta & a $a$ & Afra al & & $\begin{array}{l}\text { Gala al } \\
142,14\end{array}$ & $\begin{array}{l}a \\
2,65\end{array}$ & Granola a & $\mathrm{b} a b$ & Lolita & b $a$ & Marabel & b $a b$ & Nicola 1 & $a$ & Renate & b $a$ \\
\hline & & $1,056-1,065$ & $\mathrm{~b}$ & 169,97 & 9,81 & & & 180,98 & 3,01 & 167,80 & 1,80 & 155,25 & 10,26 & 161,62 & 3,15 & 146,48 & 15,71 & 169,16 & 1,18 \\
\hline \multirow[t]{10}{*}{2006 B } & \multirow[t]{4}{*}{5 Monate Lagerung A } & $1,066-1,075$ & $\mathrm{ab}$ & 202,90 & 3,49 & 188,44 & 1,92 & 187,88 & 1,99 & 185,09 & 4,20 & 183,84 & 6,06 & 186,78 & 2,14 & 179,85 & 2,17 & 194,04 & 2,60 \\
\hline & & $1,076-1,085$ & $\mathrm{a}$ & 325,33 & 127,58 & 209,89 & 8,68 & 198,34 & 4,26 & 205,29 & 8,75 & 194,85 & 2,22 & 202,38 & 0,36 & 195,20 & 2,23 & 207,10 & 1,37 \\
\hline & & $1,086-1,095$ & $\mathrm{a}$ & 217,03 & 5,04 & 227,96 & 7,40 & & & & & 215,77 & 3,56 & & & 217,80 & 66,58 & 224,41 & 3,21 \\
\hline & & $>1,095$ & $\mathrm{a}$ & 246,22 & 6,97 & 245,02 & 2,25 & & & & & & & & & & & & \\
\hline & \multirow{6}{*}{8 Monate Lagerung A } & $<1,055$ & $\mathrm{e}$ & Adretta & a $a$ & \multicolumn{2}{|c|}{ Afra $\mathbf{b} a$} & \multicolumn{2}{|c|}{$\begin{array}{l}\text { Gala b } a \\
141,97 \quad 1,73\end{array}$} & \multicolumn{2}{|c|}{ Granola $\mathbf{b} b$} & \multicolumn{2}{|c|}{ Lolita b $a$} & \multicolumn{2}{|c|}{ Marabel b $b$} & \multicolumn{2}{|c|}{ Nicola $\mathbf{b} a$} & \multicolumn{2}{|c|}{ Renate b $a$} \\
\hline & & $1,056-1,065$ & d & & & & & 163,23 & 0,68 & 164,58 & 5,16 & 174,50 & 3,86 & 164,60 & 0,56 & 173,20 & 8,16 & 172,15 & 0,42 \\
\hline & & $1,066-1,075$ & $\mathrm{c}$ & 207,20 & 1,16 & 178,00 & 1,15 & 198,36 & 3,89 & 180,27 & 6,64 & 175,27 & 7,48 & 183,94 & 2,08 & 170,11 & 18,26 & 186,27 & 5,69 \\
\hline & & $1,076-1,085$ & $\mathrm{~b}$ & 219,55 & 0,66 & 225,78 & 2,43 & & & 198,69 & 8,15 & 197,48 & 5,20 & 199,07 & 8,81 & 198,46 & 3,72 & 203,34 & 3,65 \\
\hline & & $1,086-1,095$ & $\mathrm{a}$ & 254,31 & 3,99 & 227,20 & 0,76 & & & & & & & 216,67 & 1,30 & & & 229,39 & 2,79 \\
\hline & & $>1,095$ & $\mathrm{a}$ & 247,50 & 3,06 & 237,76 & 1,28 & & & & & & & & & & & & \\
\hline
\end{tabular}


Tabelle 10.48 Trockenmassekonzentration [Mittelwert $(\bar{x})$ und Standartabweichung $\left(\sigma_{\mathrm{x}}\right)$ ] $\left(\mathrm{g} \mathrm{kg}^{-1} \mathrm{FM}\right)$ in Sorten und Knollen verschiedener spezifischer Dichten $\left(\mathrm{kg} \mathrm{L}^{-1}\right)$ nach der Ernte 2007 und nach fünf und acht Monaten Lagerung

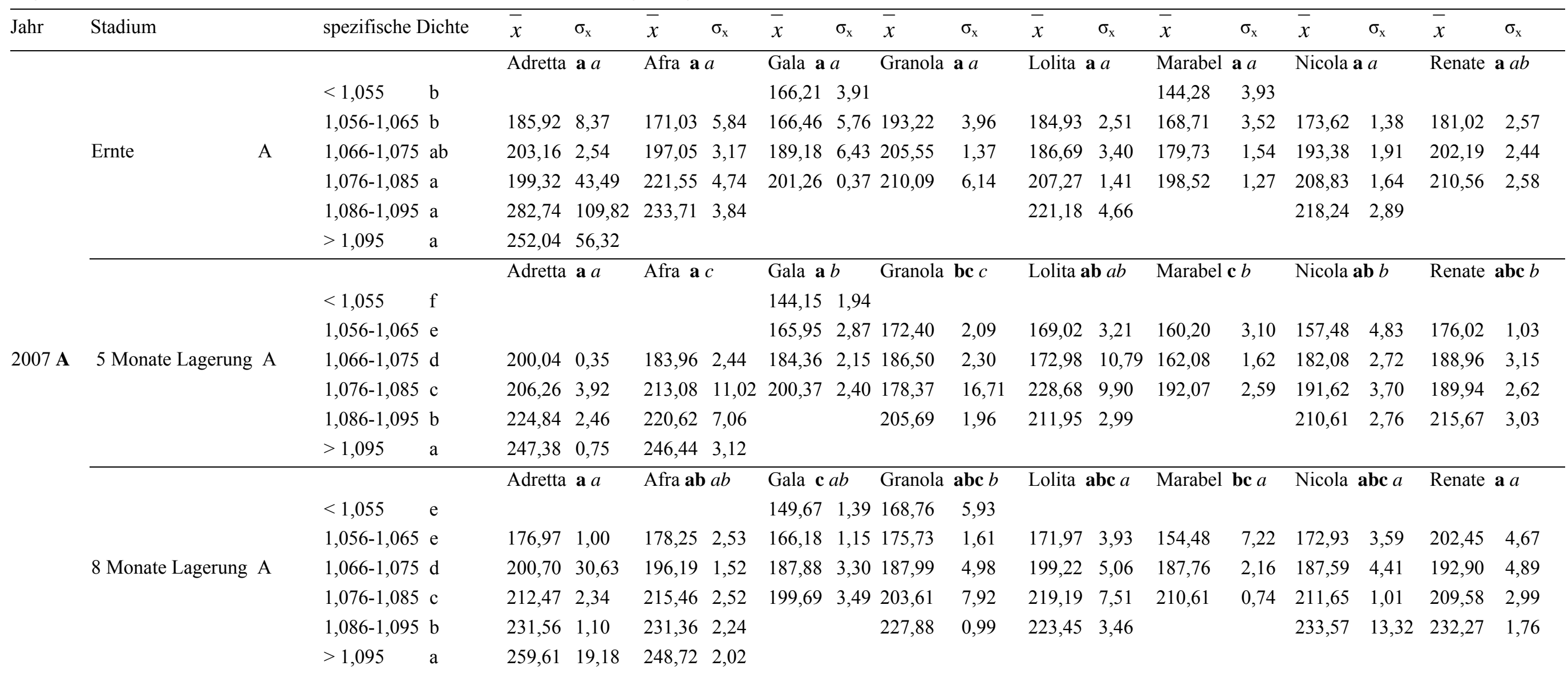


Tabelle 10.49 Stärkekonzentration [Mittelwert $(\bar{x})$ und Standartabweichung $\left(\sigma_{\mathrm{x}}\right)$ ] $\left(\mathrm{g} \mathrm{kg}^{-1} \mathrm{FM}\right)$ in Sorten und Knollen verschiedener spezifischer Dichten $\left(\mathrm{kg} \mathrm{L}^{-1}\right)$ nach der Ernte 2005 und nach fünf und acht Monaten Lagerung

\begin{tabular}{|c|c|c|c|c|c|c|c|c|c|c|c|c|c|c|c|c|c|c|c|c|}
\hline Jahr & \multicolumn{2}{|l|}{ Stadium } & \multicolumn{2}{|c|}{ spezifische Dichte } & \multirow{3}{*}{$\frac{\bar{x}}{\text { Adretta }}$} & \multirow{3}{*}{$\frac{\sigma_{\mathrm{x}}}{a}$} & \multirow{2}{*}{\multicolumn{2}{|c|}{$\begin{array}{ll}\bar{x} & \sigma_{\mathrm{x}} \\
\text { Afra } & \text { a } a\end{array}$}} & \multirow{2}{*}{$\begin{array}{l}\bar{x} \\
\text { Gala a }\end{array}$} & \multirow{2}{*}{$\frac{\sigma_{\mathrm{x}}}{a}$} & \multirow{3}{*}{$\frac{\bar{x}}{\text { Granola }}$} & \multirow{3}{*}{$\begin{array}{l}\sigma_{\mathrm{x}} \\
\mathbf{a} a\end{array}$} & $\bar{x}$ & $\sigma_{\mathrm{x}}$ & \multirow{2}{*}{\multicolumn{2}{|c|}{$\begin{array}{ll}\bar{x} & \sigma_{\mathrm{x}} \\
\text { Marabel } \mathbf{a} a\end{array}$}} & \multirow{3}{*}{$\frac{\bar{x}}{\text { Nicola }}$} & \multirow{3}{*}{$\frac{\sigma_{\mathrm{x}}}{\mathrm{a} a}$} & \multirow{3}{*}{$\frac{\bar{x}}{\text { Renate }}$} & \multirow{3}{*}{$\frac{\sigma_{\mathrm{x}}}{\mathrm{a} a}$} \\
\hline & & & & & & & & & & & & & & $a$ & & & & & & \\
\hline & & & $<1,055$ & $\mathrm{~d}$ & & & & & 79,33 & 0,09 & & & & & 89,77 & 0,11 & & & & \\
\hline & & & $1,056-1,065$ & $\mathrm{~d}$ & 99,14 & 0,04 & 99,05 & 0,08 & 98,22 & 0,28 & 109,12 & 0,22 & & & 98,61 & 0,06 & 104,30 & 0,05 & 98,53 & 0,29 \\
\hline & \multirow[t]{7}{*}{ Ernte } & A & $1,066-1,075$ & $\mathrm{c}$ & 123,14 & 0,15 & 118,08 & 0,12 & 116,12 & 0,12 & 130,51 & 0,24 & 115,58 & 0,12 & 121,20 & 0,16 & 125,00 & 0,15 & 121,73 & 0,35 \\
\hline & & & $1,076-1,085$ & $\mathrm{~b}$ & 138,13 & 2,15 & 150,35 & 0,72 & 128,97 & 0,21 & 142,29 & 0,14 & 136,28 & 0,09 & & & 140,28 & 0,12 & 140,11 & 0,14 \\
\hline & & & $1,086-1,095$ & $\mathrm{a}$ & 168,56 & 0,17 & 161,03 & 0,50 & & & & & 144,90 & 0,15 & & & & & & \\
\hline & & & $>1,095$ & $\mathrm{a}$ & & & 175,60 & 2,54 & & & & & & & & & & & & \\
\hline & & & & & Adretta & $\mathbf{a b} b$ & Afra $\mathbf{a b}$ & & Gala $\mathbf{b}$ & & Granola & a $a$ & Lolita & $b$ & Marabel & b $b$ & Nicola & b $b$ & Renate & ab $a$ \\
\hline & & & $<1,055$ & $\mathrm{~b}$ & & & & & 56,30 & 0,96 & & & & & 56,03 & 0,92 & 70,18 & 0,08 & & \\
\hline & & & $1,056-1,065$ & $\mathrm{~b}$ & 89,56 & 0,07 & 102,03 & 0,03 & 81,18 & 0,22 & 100,54 & 0,40 & 82,85 & 0,87 & 73,34 & 0,48 & 84,51 & 0,38 & 88,82 & 0,29 \\
\hline \multirow[t]{11}{*}{$2005 \mathrm{C}$} & \multirow{4}{*}{\multicolumn{2}{|c|}{5 Monate Lagerung B }} & $1,066-1,075$ & $\mathrm{a}$ & 109,60 & 0,69 & 118,33 & 0,17 & 90,21 & 1,28 & 190,87 & 12,43 & 105,13 & 0,65 & 91,95 & 0,58 & 97,67 & 0,19 & 105,28 & 0,80 \\
\hline & & & $1,076-1,085$ & $\mathrm{a}$ & 153,04 & 1,49 & 141,47 & 0,20 & 117,51 & 0,59 & 134,39 & 0,32 & 110,99 & 1,34 & 98,90 & 0,08 & 118,33 & 0,36 & 120,66 & 0,27 \\
\hline & & & $1,086-1,095$ & $\mathrm{a}$ & 153,86 & 0,36 & 161,58 & 0,20 & & & & & 138,34 & 0,40 & & & 125,49 & 0,61 & 135,20 & 0,94 \\
\hline & & & $>1,095$ & $\mathrm{a}$ & & & 175,02 & 0,34 & & & & & & & & & & & & \\
\hline & \multirow{7}{*}{\multicolumn{2}{|c|}{8 Monate Lagerung $\mathrm{C}$}} & & & \multicolumn{2}{|c|}{ Adretta $\mathbf{a b} b$} & \multicolumn{2}{|c|}{ Afra $\mathbf{a} b$} & \multicolumn{2}{|c|}{ Gala b $c$} & Granola & $\mathbf{a b} b$ & \multirow[t]{2}{*}{ Lolita } & $b$ & Marabel & b $c$ & Nicola & b $b$ & Renate & $\mathbf{a b} b$ \\
\hline & & & $<1,055$ & $\mathrm{~d}$ & & & & & 50,36 & 0,23 & & & & & 48,08 & 0,56 & & & & \\
\hline & & & $1,0556-1,065$ & $\mathrm{~d}$ & 84,93 & 0,72 & 94,60 & 0,44 & 71,85 & 0,48 & 76,29 & 1,52 & 72,44 & 0,37 & 61,65 & 0,56 & 63,12 & 0,15 & 82,96 & 0,65 \\
\hline & & & $1,066-1,075$ & $\mathrm{c}$ & 109,70 & 0,63 & 119,17 & 0,48 & 86,01 & 0,43 & 106,35 & 0,63 & 87,60 & 0,27 & 78,23 & 0,08 & 107,25 & 0,23 & 103,97 & 0,42 \\
\hline & & & $1,076-1,085$ & $\mathrm{~b}$ & 123,66 & 0,38 & 129,89 & 0,52 & 96,30 & 0,64 & 130,34 & 1,89 & 114,68 & 0,16 & 91,03 & 0,56 & 108,85 & 0,44 & 113,32 & 0,22 \\
\hline & & & $1,086-1,095$ & $\mathrm{ab}$ & 133,28 & 0,79 & 146,23 & 0,57 & & & & & 113,94 & 0,31 & & & & & & \\
\hline & & & $>1,095$ & $\mathrm{a}$ & & & 156,34 & 1,52 & & & & & & & & & & & & \\
\hline
\end{tabular}


Tabelle 10.50 Stärkekonzentration [Mittelwert $(\bar{x})$ und Standartabweichung $\left(\sigma_{\mathrm{x}}\right)$ ] $\left(\mathrm{g} \mathrm{kg}^{-1} \mathrm{FM}\right)$ in Sorten und Knollen verschiedener spezifischer Dichten $\left(\mathrm{kg} \mathrm{L}^{-1}\right)$ nach der Ernte 2006 und nach fünf und acht Monaten Lagerung

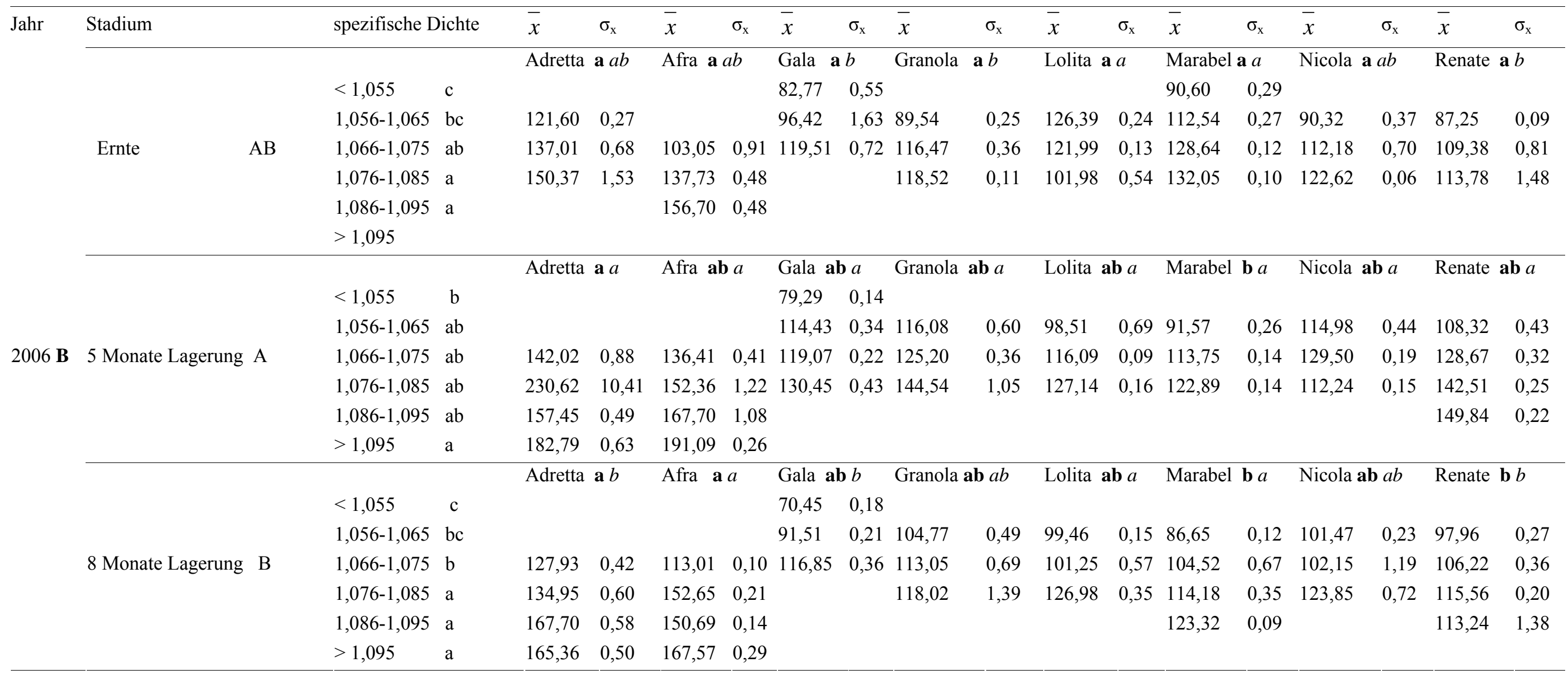


Tabelle 10.51 Stärkekonzentration [Mittelwert $(\bar{x})$ und Standartabweichung $\left(\sigma_{\mathrm{x}}\right)$ ] $\left(\mathrm{g} \mathrm{kg}^{-1} \mathrm{FM}\right)$ in Sorten und Knollen verschiedener spezifischer Dichten $\left(\mathrm{kg} \mathrm{L}^{-1}\right)$ nach der Ernte 2007 und nach fünf und acht Monaten Lagerung

\begin{tabular}{|c|c|c|c|c|c|c|c|c|c|c|c|c|c|c|c|c|c|c|}
\hline Jahr & Stadium & spezifische Dichte & $\bar{x}$ & $\sigma_{\mathrm{x}}$ & $\bar{x}$ & $\sigma_{\mathrm{x}}$ & $\bar{x}$ & $\sigma_{\mathrm{x}}$ & $\bar{x}$ & $\sigma_{\mathrm{x}}$ & $\bar{x}$ & $\sigma_{\mathrm{x}}$ & $\bar{x}$ & $\sigma_{\mathrm{x}}$ & $\bar{x}$ & $\sigma_{\mathrm{x}}$ & $\bar{x}$ & $\sigma_{\mathrm{x}}$ \\
\hline & & & Adretta & a $a$ & Afra $\mathbf{a} c$ & & Gala a $a$ & & Granola & a $a$ & Lolita a & & Marabel & a $a$ & Nicola & $a$ & Renate & $a b$ \\
\hline & & $<1,055$ & & & & & 103,70 & 0,32 & & & & & 82,16 & 0,28 & & & & \\
\hline & & $1,056-1,065 \mathrm{~cd}$ & 117,92 & 0,63 & & & 107,97 & 0,37 & 130,00 & 0,13 & 122,24 & 0,47 & 101,93 & 0,35 & 111,84 & 0,42 & 117,03 & 0,21 \\
\hline & Ernte & $1,066-1,075 \mathrm{bc}$ & 135,23 & 0,28 & 124,08 & 0,44 & 121,77 & 0,94 & 142,00 & 0,09 & 128,84 & 0,21 & 115,39 & 0,50 & 126,40 & 0,21 & 134,96 & 0,17 \\
\hline & & $1,076-1,085 \mathrm{~b}$ & 136,51 & 2,96 & 150,45 & 1,00 & 130,73 & 0,21 & 144,94 & 0,54 & 152,42 & 0,43 & 135,94 & 0,41 & 134,63 & 0,11 & 148,50 & 0,48 \\
\hline & & $\begin{array}{l}1,086-1,095 \mathrm{a} \\
>1,095\end{array}$ & 194,59 & 7,54 & 151,33 & 0,62 & & & & & & & & & & & & \\
\hline & & & Adretta & a $a$ & Afra $\mathbf{a b}$ & & Gala $\mathbf{b} a$ & & Granola & ab $a$ & Lolita ab & & Marabel & $c b$ & Nicola a & c $a$ & Renate a & $a b$ \\
\hline & & $<1,055 \quad \mathrm{~d}$ & & & & & 79,95 & 0,20 & & & & & & & & & & \\
\hline & & $1,056-1,065 \mathrm{~d}$ & & & & & 99,13 & 0,60 & 103,92 & 1,60 & 103,27 & 0,55 & & & 96,15 & 0,48 & 112,74 & 0,31 \\
\hline $2007 \mathbf{A}$ & 5 Monate Lagerung B & $1,066-1,075 \mathrm{c}$ & 139,29 & 0,36 & 123,35 & 0,29 & 114,54 & 0,40 & 127,16 & 0,65 & 116,31 & 0,71 & 91,73 & 0,61 & 115,58 & 0,30 & 131,29 & 0,15 \\
\hline & & $1,076-1,085 \mathrm{~b}$ & 140,48 & 0,68 & 148,80 & 0,76 & 121,14 & 0,55 & 120,33 & 1,05 & 152,68 & 0,71 & 118,27 & 0,40 & 130,02 & 0,56 & 137,14 & 0,21 \\
\hline & & $1,086-1,095 \mathrm{~b}$ & 160,53 & 0,28 & 160,89 & 1,01 & & & 141,44 & 0,13 & 148,78 & 0,76 & & & 146,09 & 0,16 & 148,20 & 0,34 \\
\hline & & $>1,095 \quad a$ & 187,87 & 0,18 & 181,27 & 0,54 & & & & & & & & & & & & \\
\hline & & $<1,055$ & Adretta & a $a$ & Afra a $a$ & & $\begin{array}{l}\text { Gala c al } \\
84,80\end{array}$ & 0,14 & $\begin{array}{l}\text { Granola } \\
108,58\end{array}$ & $\begin{array}{l}\mathbf{a} a \\
0,45\end{array}$ & Lolita $\mathbf{a b}$ & $c a b$ & Marabel & $a b$ & Nicola & bc $a$ & Renate a & \\
\hline & & $1,056-1,065 \mathrm{~d}$ & 115,07 & 0,27 & & & 103,38 & 0,39 & 123,79 & 0,72 & 106,86 & 0,65 & 73,86 & 1,11 & 108,05 & 0,61 & 131,59 & 0,57 \\
\hline & 8 Monate Lagerung $\mathrm{AB}$ & $1,066-1,075 \mathrm{~cd}$ & 136,99 & 2,17 & 132,88 & 0,40 & 109,90 & 2,08 & 133,62 & 0,80 & 129,41 & 0,62 & 118,44 & 0,41 & 121,82 & 0,37 & 128,85 & 0,21 \\
\hline & & $1,076-1,085 \mathrm{bc}$ & 154,75 & 0,27 & 153,88 & 0,81 & 94,60 & 0,31 & 150,58 & 1,30 & 148,47 & 0,66 & 125,52 & 0,91 & 139,96 & 0,15 & 142,22 & 0,48 \\
\hline & & $1,086-1,095 \mathrm{ab}$ & 173,68 & 0,67 & 164,06 & 0,27 & & & & & 152,26 & 0,33 & & & 161,11 & 1,38 & 156,55 & 0,32 \\
\hline & & $>1,095$ & 171,80 & 3,74 & 185,78 & 0,33 & & & & & & & & & & & & \\
\hline
\end{tabular}


Tabelle 10.52 Konzentration des trockenen Zellwandmaterials [Mittelwert $(\bar{x})$ und Standartabweichung $\left(\sigma_{\mathrm{x}}\right)$ ] $\left(\mathrm{g} \mathrm{kg}{ }^{-1} \mathrm{FM}\right)$ in Sorten und Knollen verschiedener spezifischer Dichten $\left(\mathrm{kg} \mathrm{L}^{-1}\right)$ nach der Ernte 2005 und nach fünf und acht Monaten Lagerung

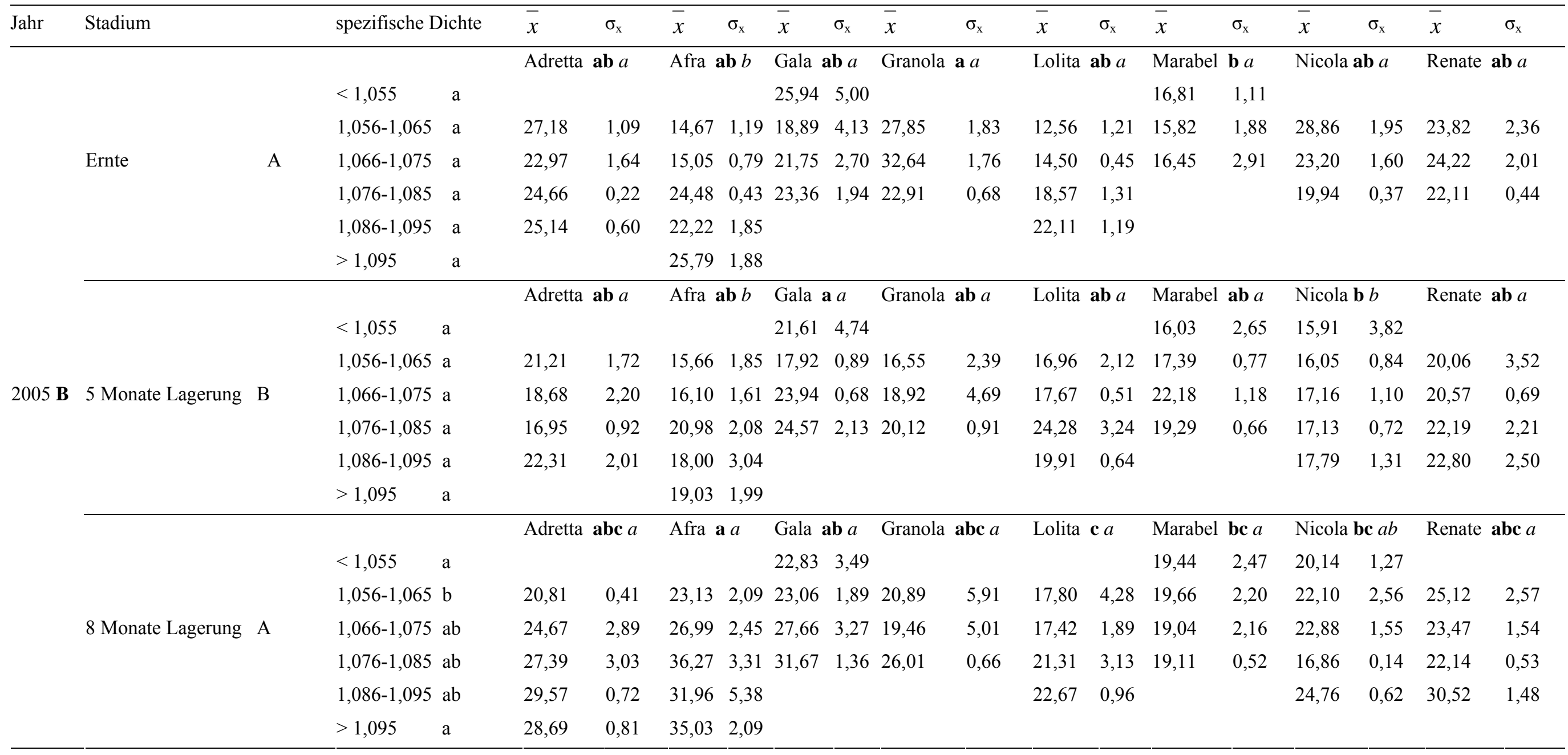


Tabelle 10.53 Konzentration des trockenen Zellwandmaterials [Einzelwerte] ( $\left.\mathrm{g} \mathrm{kg}^{-1} \mathrm{FM}\right)$ in Sorten und Knollen verschiedener spezifischer Dichten (kg L ${ }^{-1}$ ) nach der Ernte 2006 und nach fünf und acht Monaten Lagerung

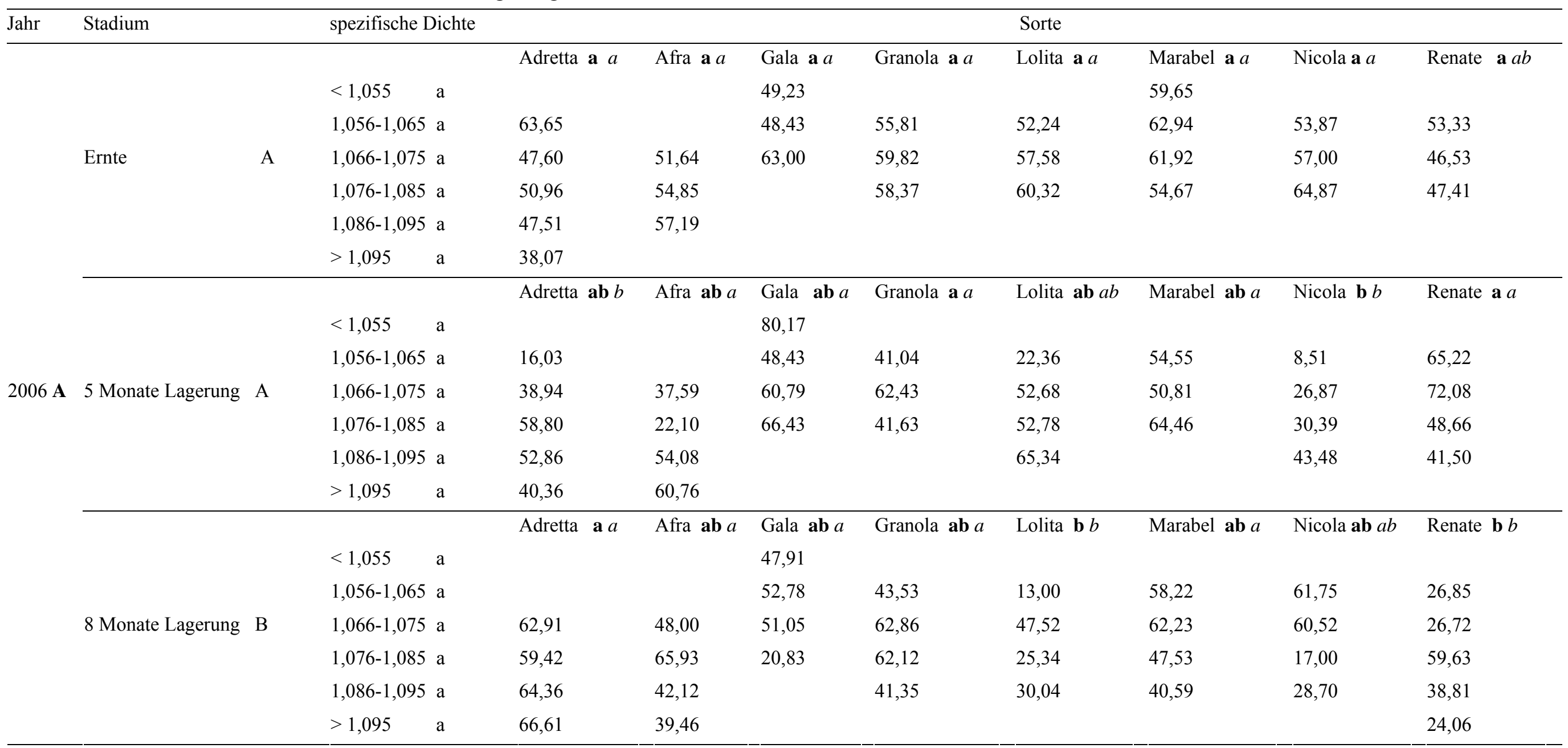


Tabelle 10.54 Konzentration des trockenen Zellwandmaterials [Einzelwerte] ( $\left.\mathrm{g} \mathrm{kg}^{-1} \mathrm{FM}\right)$ in Sorten und Knollen verschiedener spezifischer Dichten (kg L ${ }^{-1}$ ) nach der Ernte 2007 und nach fünf und acht Monaten Lagerung

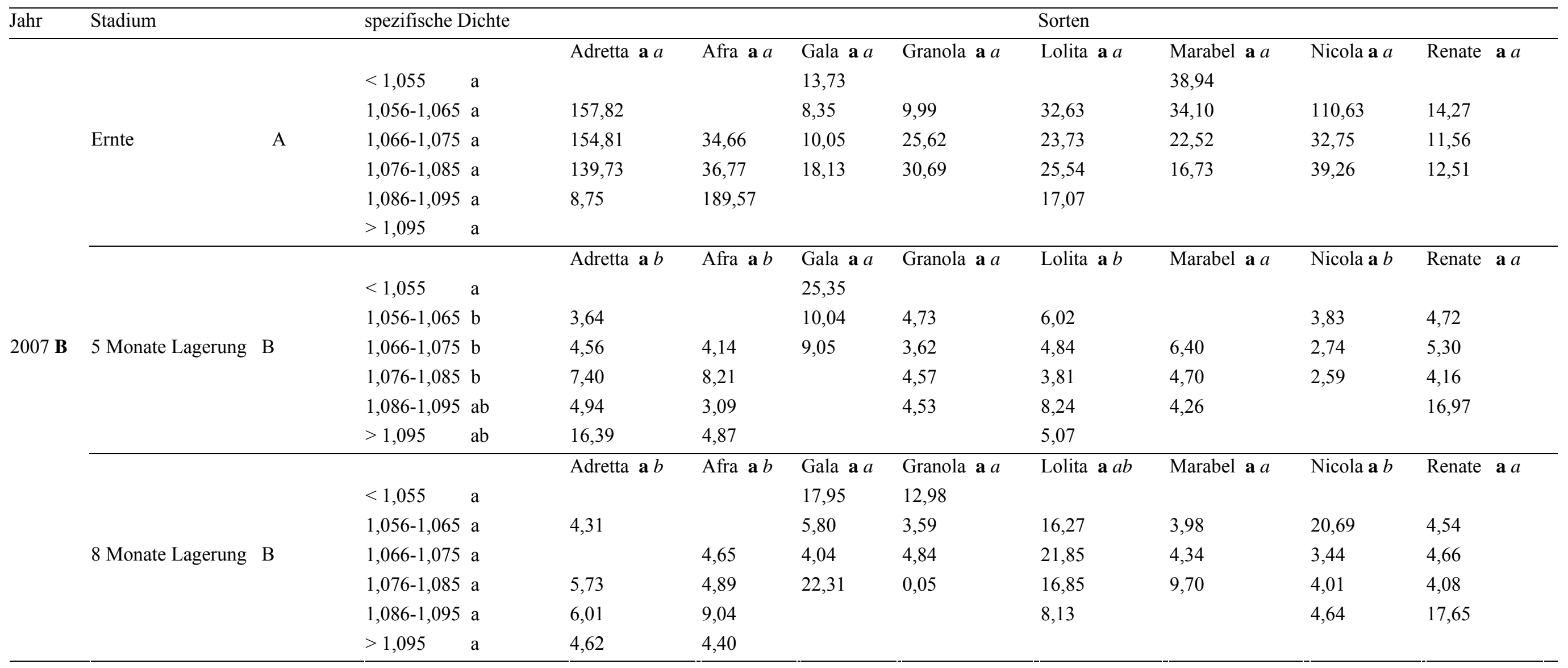


Tabelle 10.55 Pektinkonzentration [Mittelwert $(\bar{x})$ und Standartabweichung $\left(\sigma_{\mathrm{x}}\right)$ ] $\left(\mathrm{g} \mathrm{kg}^{-1} \mathrm{FM}\right)$ in Sorten und Knollen verschiedener spezifischer Dichten $\left(\mathrm{kg} \mathrm{L}^{-1}\right)$ nach der Ernte 2005 und nach fünf und acht Monaten Lagerung

\begin{tabular}{|c|c|c|c|c|c|c|c|c|c|c|c|c|c|c|c|c|c|c|c|c|}
\hline Jahr & \multicolumn{2}{|l|}{ Stadium } & \multicolumn{2}{|c|}{ spezifische Dichte } & \multirow{3}{*}{$\begin{array}{l}\bar{x} \\
\text { Adretta }\end{array}$} & \multirow{2}{*}{$\begin{array}{l}\sigma_{\mathrm{x}} \\
\mathbf{a b} a\end{array}$} & & & \multirow{2}{*}{$\begin{array}{l}\bar{x} \\
\text { Gala }\end{array}$} & & \multirow{3}{*}{$\begin{array}{l}\bar{x} \\
\text { Granola }\end{array}$} & & \multirow{3}{*}{$\begin{array}{l}\bar{x} \\
\text { Lolita }\end{array}$} & \multirow{3}{*}{$\begin{array}{l}\sigma_{\mathrm{x}} \\
\mathbf{b} a\end{array}$} & \multirow{2}{*}{\multicolumn{2}{|c|}{$\begin{array}{l}\bar{x} \quad \sigma_{\mathrm{x}} \\
\text { Marabel } \mathbf{b} a\end{array}$}} & \multirow{2}{*}{\multicolumn{2}{|c|}{$\begin{array}{l}\bar{x} \quad \sigma_{\mathrm{x}} \\
\text { Nicola } \mathbf{a b} a\end{array}$}} & \multirow{3}{*}{$\begin{array}{l}\bar{x} \\
\text { Renate }\end{array}$} & \multirow{3}{*}{$\begin{array}{l}\sigma_{\mathrm{x}} \\
\mathbf{a b} a\end{array}$} \\
\hline & & & & & & & & & & & & & & & & & & & & \\
\hline & & & $<1,055$ & $\mathrm{a}$ & & & & & 4,38 & 0,79 & & & & & 2,51 & 0,20 & & & & \\
\hline & & & $1,056-1,065$ & $\mathrm{a}$ & 3,89 & 0,48 & 2,37 & 0,15 & 3,19 & 0,69 & 4,29 & 0,05 & 1,83 & 0,14 & 2,26 & 0,48 & 3,88 & 0,24 & 4,17 & 0,31 \\
\hline & \multirow[t]{7}{*}{ Ernte } & \multirow[t]{7}{*}{ A } & $1,066-1,075$ & $\mathrm{a}$ & 3,27 & 0,17 & 2,32 & 0,08 & 3,41 & 0,32 & 4,81 & 0,22 & 2,11 & 0,19 & 2,24 & 0,39 & 3,11 & 0,18 & 4,07 & 0,32 \\
\hline & & & $1,076-1,085$ & $\mathrm{a}$ & 3,65 & 0,04 & 3,59 & 0,40 & 3,62 & 0,23 & 3,25 & 0,21 & 2,56 & 0,23 & & & 2,61 & 0,06 & 3,11 & 0,23 \\
\hline & & & $1,086-1,095$ & $\mathrm{a}$ & 3,56 & 0,22 & 3,35 & 0,45 & & & & & 2,97 & 0,13 & & & & & & \\
\hline & & & $>1,095$ & $\mathrm{a}$ & & & 3,81 & 0,19 & & & & & & & & & & & & \\
\hline & & & & & Adretta & $\mathbf{a b} b$ & Afra & b $b$ & Gala & ab $a$ & Granola & $\mathbf{a b} a$ & Lolita & $\mathbf{a b} a$ & Marabe & $\mathbf{a b} a$ & Nicola & b $b$ & Renate & a $a$ \\
\hline & & & $<1,055$ & $\mathrm{a}$ & & & & & 3,01 & 0,39 & & & & & 2,23 & 0,27 & 2,18 & 0,56 & & \\
\hline & & & $1,056-1,065$ & $\mathrm{a}$ & 2,58 & 0,33 & 2,27 & 0,34 & 2,31 & 0,23 & 2,37 & 0,33 & 2,37 & 0,41 & 2,30 & 0,08 & 2,19 & 0,15 & 2,99 & 0,37 \\
\hline \multirow[t]{11}{*}{2005 A } & \multirow[t]{4}{*}{5 Monate Lagerung } & \multirow[t]{4}{*}{ B } & $1,066-1,075$ & $\mathrm{a}$ & 2,24 & 0,19 & 2,32 & 0,28 & 2,93 & 0,12 & 2,59 & 0,86 & 2,48 & 0,17 & 2,83 & 0,27 & 2,19 & 0,22 & 2,90 & 0,12 \\
\hline & & & $1,076-1,085$ & $\mathrm{a}$ & 1,98 & 0,06 & 2,55 & 0,18 & 2,95 & 0,09 & 2,65 & 0,13 & 3,31 & 0,38 & 2,48 & 0,14 & 2,25 & 0,07 & 3,08 & 0,10 \\
\hline & & & $1,086-1,095$ & $\mathrm{a}$ & 2,50 & 0,24 & 2,19 & 0,40 & & & & & 2,51 & 0,12 & & & 2,38 & 0,19 & 3,07 & 0,29 \\
\hline & & & $>1,095$ & $\mathrm{a}$ & & & 2,26 & 0,20 & & & & & & & & & & & & \\
\hline & \multirow{7}{*}{8 Monate Lagerung } & \multirow{7}{*}{ A } & & & Adretta & bc $b$ & Afra & $\mathbf{a b} a$ & Gala & a $a$ & Granola & c $a$ & Lolita & c $a$ & Marabe & c $a$ & Nicola & abc $a$ & Renate & a $a$ \\
\hline & & & $<1,055$ & $\mathrm{a}$ & & & & & 3,27 & 0,45 & & & & & 2,71 & 0,28 & 3,07 & 0,24 & & \\
\hline & & & $1,056-1,065$ & $\mathrm{a}$ & 2,57 & 0,31 & 3,01 & 0,25 & 3,27 & 0,24 & 2,56 & 0,40 & 2,43 & 0,64 & 2,72 & 0,31 & 3,15 & 0,46 & 3,66 & 0,37 \\
\hline & & & $1,066-1,075$ & $\mathrm{a}$ & 2,82 & 0,34 & 3,37 & 0,29 & 3,81 & 0,42 & 2,32 & 0,48 & 2,37 & 0,28 & 2,60 & 0,03 & 3,29 & 0,33 & 3,60 & 0,13 \\
\hline & & & $1,076-1,085$ & $\mathrm{a}$ & 3,03 & 0,43 & 3,76 & 0,51 & 4,12 & 0,15 & 3,03 & 0,23 & 2,73 & 0,18 & 2,58 & 0,05 & 2,53 & 0,11 & 3,43 & 0,30 \\
\hline & & & $1,086-1,095$ & $\mathrm{a}$ & 3,27 & 0,15 & 3,79 & 0,57 & & & & & 2,78 & 0,15 & & & 3,33 & 0,31 & 4,09 & 0,25 \\
\hline & & & $>1,095$ & $\mathrm{a}$ & 3,43 & 0,07 & 3,93 & 0,35 & & & & & & & & & & & & \\
\hline
\end{tabular}


Tabelle 10.56 Pektinkonzentration [Einzelwerte] $\left(\mathrm{g} \mathrm{kg}^{-1} \mathrm{FM}\right)$ in Sorten und Knollen verschiedener spezifischer Dichten $\left(\mathrm{kg} \mathrm{L}^{-1}\right)$ nach der Ernte 2006 und nach fünf und acht Monaten Lagerung

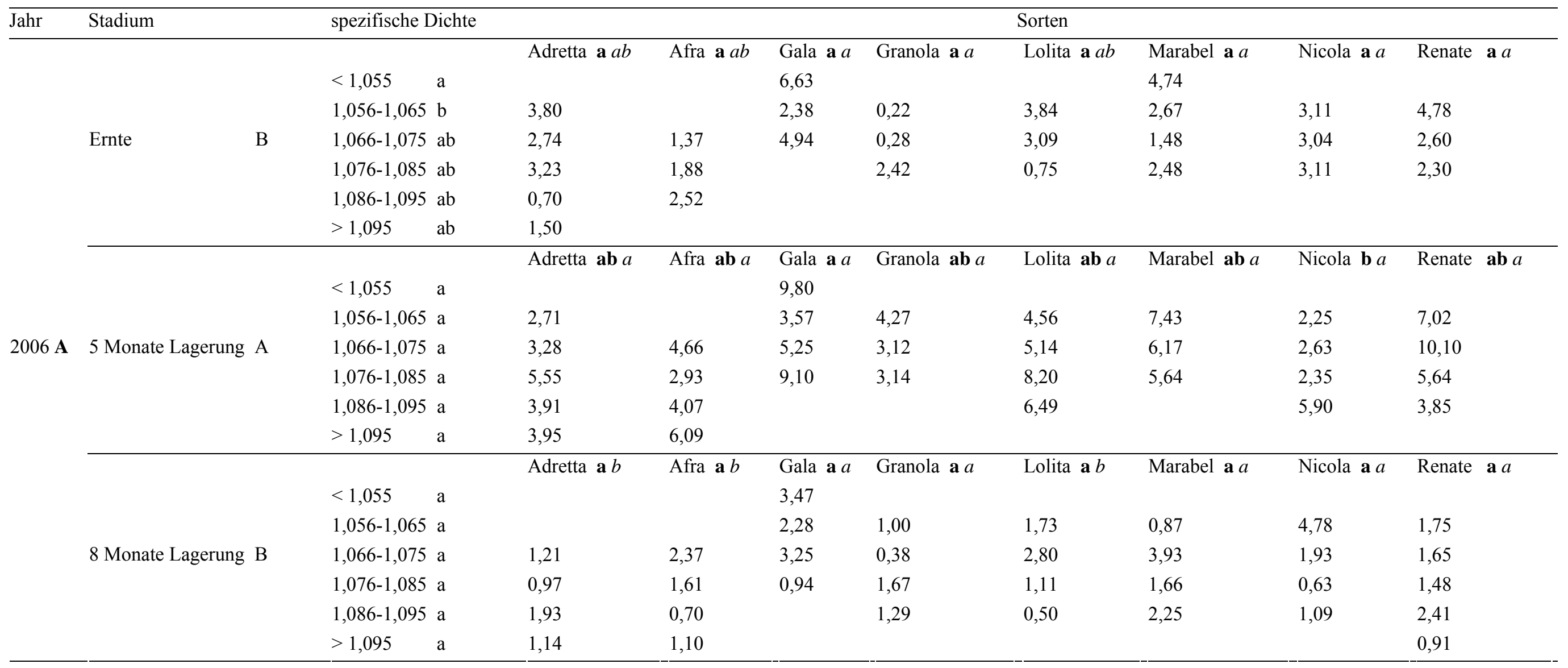


Tabelle 10.57 Pektinkonzentration [Einzelwerte] $\left(\mathrm{g} \mathrm{kg}^{-1} \mathrm{FM}\right)$ in Sorten und Knollen verschiedener spezifischer Dichten $\left(\mathrm{kg} \mathrm{L}^{-1}\right)$ nach der Ernte 2007 und nach fünf und acht Monaten Lagerung

\begin{tabular}{|c|c|c|c|c|c|c|c|c|c|c|}
\hline Jahr & Stadium & spezifische Dichte & & & & & Sorten & & & \\
\hline & & $<1,055 \quad a$ & Adretta a $a$ & Afra a $a$ & $\begin{array}{l}\text { Gala a } a \\
1,13\end{array}$ & Granola a $a$ & Lolita a $a$ & $\begin{array}{l}\text { Marabel a } a \\
2,29\end{array}$ & Nicola a $a$ & Renate a $a$ \\
\hline & & $1,056-1,065 \mathrm{a}$ & 9,96 & 2,64 & 0,44 & 0,61 & 2,07 & 3,57 & 5,56 & 1,38 \\
\hline & Ernte & $1,066-1,075$ a & 14,05 & 0,27 & 0,74 & 1,26 & 2,46 & 1,12 & 3,31 & 1,28 \\
\hline & & $1,076-1,085$ a & 10,28 & 15,53 & 1,52 & 2,60 & 2,64 & 2,03 & 2,77 & 0,92 \\
\hline & & $1,086-1,095 \mathrm{a}$ & 0,90 & & & & 2,36 & & & \\
\hline & & $>1,095$ & & & & & & & & \\
\hline & & $<1,055$ & Adretta $\mathbf{a} b$ & Afra a $a$ & $\begin{array}{l}\text { Gala a } a \\
1,39\end{array}$ & Granola a $a$ & Lolita a $c$ & Marabel a $a$ & Nicola a $b$ & Renate a $a b$ \\
\hline & & $1,056-1,065 \mathrm{a}$ & 0,30 & 0,36 & 0,82 & 0,40 & 0,31 & & 0,39 & 0,37 \\
\hline \multirow[t]{10}{*}{2007 B } & 5 Monate Lagerung B & $1,066-1,075 \mathrm{a}$ & 0,23 & 0,50 & 0,68 & 0,25 & 0,35 & 0,29 & 0,22 & 0,43 \\
\hline & & $1,076-1,085$ a & 0,41 & 0,23 & & 0,36 & 0,30 & 0,27 & 0,16 & 0,30 \\
\hline & & $1,086-1,095$ a & 0,56 & 0,38 & & 0,47 & 0,45 & 0,40 & & 1,39 \\
\hline & & $>1,095$ & 1,58 & & & & 0,51 & & & \\
\hline & & $<1,055$ & Adretta $\mathbf{a} b$ & Afra a $a$ & $\begin{array}{l}\text { Gala a } a \\
1,64\end{array}$ & $\begin{array}{l}\text { Granola a } a \\
0,81\end{array}$ & Lolita a $b$ & Marabel a $a$ & Nicola a $b$ & Renate $\mathbf{a} b$ \\
\hline & & $1,056-1,065 \mathrm{a}$ & 0,36 & 0,00 & 0,56 & 0,23 & 0,78 & 0,36 & 1,34 & 0,45 \\
\hline & 8 Monate Lagerung $\mathrm{B}$ & $1,066-1,075$ a & & 0,39 & 0,41 & 0,31 & 0,96 & 0,42 & 0,22 & 0,64 \\
\hline & & $1,076-1,085 \mathrm{a}$ & 0,54 & 0,49 & 2,43 & 0,00 & 0,82 & 0,77 & 0,37 & 0,51 \\
\hline & & $1,086-1,095 \mathrm{a}$ & 0,37 & 0,79 & & & 0,52 & & 0,38 & 0,72 \\
\hline & & $>1,095$ & 0,37 & 0,41 & & & & & & \\
\hline
\end{tabular}


Tabelle10.58 Pektinkonzentration [Mittelwert $(\bar{x})$ und Standartabweichung $\left(\sigma_{\mathrm{x}}\right)$ der Daten aus 2005, Einzelwerte der Daten aus 2006 und 2007 ] $\left(\mathrm{g} \mathrm{kg}^{-1} \mathrm{FM}\right)$ im Schalen- und Markgewebe nach der Ernte 2005, 2006 und 2007 und nach fünf und acht Monaten Lagerung der Sorten, deren Knollen nicht nach spezifischen Dichten getrennt wurden

\begin{tabular}{|c|c|c|c|c|c|c|c|c|c|c|c|c|c|c|c|c|c|c|c|}
\hline Jahr & Stadium & & Gewebe & $\bar{x}$ & $\sigma_{\mathrm{x}}$ & $\bar{x}$ & $\sigma_{\mathrm{x}}$ & $\bar{x}$ & $\sigma_{\mathrm{x}}$ & $\overline{\bar{x}}$ & $\sigma_{\mathrm{x}}$ & $\bar{x}$ & $\sigma_{\mathrm{x}}$ & $\bar{x}$ & $\sigma_{\mathrm{x}}$ & $\bar{x}$ & $\sigma_{\mathrm{x}}$ & $\overline{\bar{x}}$ & $\sigma_{\mathrm{x}}$ \\
\hline \multirow{9}{*}{2005 A } & \multirow{3}{*}{ Ernte } & \multirow{3}{*}{ A } & & Adretta & $\mathbf{a}$ & Afra & $\mathbf{a}$ & Gala & $\mathbf{a}$ & Granola & $\mathbf{a}$ & Lolita & $\mathbf{a}$ & \multicolumn{2}{|c|}{ Marabel a } & Nicola & $\mathbf{a}$ & Renate & $\mathbf{a}$ \\
\hline & & & Mark b & 3,73 & 0,20 & 2,22 & 0,18 & 2,90 & 1,28 & 1,86 & 0,10 & 1,93 & 0,49 & 2,66 & 0,15 & 2,82 & 0,92 & 2,89 & 0,15 \\
\hline & & & Schale a & 5,72 & 1,02 & 5,37 & 0,71 & 6,50 & 1,08 & 6,39 & 0,93 & 5,56 & 0,95 & 6,41 & 0,95 & 5,85 & 0,98 & 6,30 & 1,03 \\
\hline & & & & \multicolumn{2}{|c|}{ Adretta $\mathbf{a}$} & \multicolumn{2}{|c|}{ Afra a } & \multicolumn{2}{|c|}{ Gala a } & \multicolumn{2}{|c|}{ Granola a } & \multicolumn{2}{|c|}{ Lolita a } & \multicolumn{2}{|c|}{ Marabel a } & \multicolumn{2}{|c|}{ Nicola a } & \multicolumn{2}{|c|}{ Renate a } \\
\hline & \multirow[t]{3}{*}{5 Monate Lagerung } & \multirow[t]{3}{*}{ A } & Mark b & 1,80 & 0,09 & 1,40 & 0,34 & 2,40 & 0,23 & 1,97 & 0,81 & 1,69 & 0,29 & 1,91 & 0,24 & 2,22 & 0,16 & 2,88 & 0,32 \\
\hline & & & Schale a & 5,61 & 0,58 & 5,24 & 0,71 & 5,50 & 0,47 & 6,58 & 0,65 & 5,28 & 0,30 & 4,62 & 0,25 & 3,97 & 0,43 & 6,38 & 0,44 \\
\hline & & & & Adretta & $\mathbf{a}$ & \multicolumn{2}{|c|}{ Afra a } & \multicolumn{2}{|c|}{ Gala a } & \multicolumn{2}{|c|}{ Granola a } & \multicolumn{2}{|c|}{ Lolita a } & \multicolumn{2}{|c|}{ Marabel a } & \multicolumn{2}{|c|}{ Nicola a } & \multicolumn{2}{|c|}{ Renate a } \\
\hline & \multirow[t]{2}{*}{8 Monate Lagerung } & \multirow[t]{2}{*}{ A } & Mark b & 1,90 & 0,66 & 1,99 & 0,30 & 2,52 & 0,25 & 2,15 & 0,27 & 2,32 & 0,48 & 2,10 & 0,15 & 3,03 & 0,49 & 3,31 & 0,35 \\
\hline & & & Schale a & 5,97 & 0,98 & 5,66 & 0,83 & 6,98 & 0,49 & 5,07 & 0,36 & 5,04 & 0,33 & 5,17 & 0,47 & 5,39 & 0,21 & 7,14 & 1,47 \\
\hline \multirow{4}{*}{2006 A } & \multirow[t]{2}{*}{ Ernte } & \multirow[t]{2}{*}{ A } & Mark b & $\begin{array}{l}\text { Adretta } \\
1,05\end{array}$ & \multirow[t]{2}{*}{ a $a$} & \multirow{2}{*}{\multicolumn{2}{|c|}{$\begin{array}{l}\text { Afra a } a \\
1,82 \\
4,05\end{array}$}} & \multirow{2}{*}{\multicolumn{2}{|c|}{$\begin{array}{l}\text { Gala a } a \\
2,04 \\
10,92\end{array}$}} & \multirow{2}{*}{\multicolumn{2}{|c|}{$\begin{array}{l}\text { Granola a } a \\
2,65 \\
13,93\end{array}$}} & \multirow{2}{*}{\multicolumn{2}{|c|}{$\begin{array}{l}\text { Lolita a } a \\
1,21 \\
12,07\end{array}$}} & $\begin{array}{l}\text { Marabel } \\
2,39\end{array}$ & a $a$ & $\begin{array}{l}\text { Nicola } \\
2,01\end{array}$ & a $a$ & $\begin{array}{l}\text { Renate } \\
1,55\end{array}$ & $a$ \\
\hline & & & Schale a & 1,88 & & & & & & & & & & 10,22 & & 11,33 & & 10,65 & \\
\hline & 5 Monate Lagerung & A & $\begin{array}{ll}\text { Mark } & b \\
\text { Schale } & a\end{array}$ & $\begin{array}{l}\text { Adretta } \\
2,67 \\
10,87\end{array}$ & a $a$ & $\begin{array}{l}\text { Afra } \\
2,93 \\
7,58 \\
\end{array}$ & & $\begin{array}{l}\text { Gala } \\
4,99 \\
10,98 \\
\end{array}$ & $a$ & $\begin{array}{l}\text { Granola } \\
0,85 \\
11,28\end{array}$ & a $a$ & $\begin{array}{l}\text { Lolita } \\
4,73 \\
11,87 \\
\end{array}$ & a $a$ & $\begin{array}{l}\text { Marabel } \\
2,71 \\
11,86\end{array}$ & a $a$ & $\begin{array}{l}\text { Nicola } \\
4,07 \\
12,44 \\
\end{array}$ & a $a$ & $\begin{array}{l}\text { Renate } \\
3,42 \\
17,57 \\
\end{array}$ & $a$ \\
\hline & 8 Monate Lagerung & B & $\begin{array}{ll}\text { Mark } & b \\
\text { Schale } & a\end{array}$ & $\begin{array}{l}\text { Adretta } \\
0,50 \\
1,74\end{array}$ & a $a$ & $\begin{array}{l}\text { Afra } \\
1,72 \\
3,90 \\
\end{array}$ & & $\begin{array}{l}\text { Gala } \\
0,91 \\
14,55\end{array}$ & $a$ & $\begin{array}{l}\text { Granola } \\
0,90 \\
4,38\end{array}$ & a $a$ & $\begin{array}{l}\text { Lolita } \\
1,78 \\
3,01 \\
\end{array}$ & a $a$ & $\begin{array}{l}\text { Marabel } \\
1,96 \\
1,40 \\
\end{array}$ & a $a$ & $\begin{array}{l}\text { Nicola } \\
0,89 \\
0,64 \\
\end{array}$ & a $a$ & $\begin{array}{l}\text { Renate } \\
1,06 \\
5,24 \\
\end{array}$ & a $a$ \\
\hline & Ernte & A & $\begin{array}{ll}\text { Mark a } & \text { a } \\
\text { Schale }\end{array}$ & $\begin{array}{l}\text { Adretta } \\
10,97 \\
8,03\end{array}$ & a $a$ & $\begin{array}{l}\text { Afra } \\
1,41 \\
\end{array}$ & a $a$ & $\begin{array}{l}\text { Gala } \\
2,72 \\
6,41 \\
\end{array}$ & a $a$ & $\begin{array}{l}\text { Granola } \\
0,59 \\
3,69\end{array}$ & a $a$ & $\begin{array}{l}\text { Lolita } \\
0,54 \\
3,72 \\
\end{array}$ & a $a$ & $\begin{array}{l}\text { Marabel } \\
6,95 \\
3,90\end{array}$ & a $a$ & $\begin{array}{l}\text { Nicola } \\
6,83 \\
8,88 \\
\end{array}$ & a $a$ & $\begin{array}{l}\text { Renate } \\
4,99 \\
5,91 \\
\end{array}$ & a $a$ \\
\hline 2007 B & 5 Monate Lagerung & $\mathrm{B}$ & $\begin{array}{ll}\text { Mark a } & \text { a } \\
\text { Schale }\end{array}$ & $\begin{array}{l}\text { Adretta } \\
0,34 \\
1,31 \\
\end{array}$ & a $a$ & $\begin{array}{l}\text { Afra } \\
0,39 \\
1,48 \\
\end{array}$ & a $b$ & $\begin{array}{l}\text { Gala } \\
0,68 \\
0,00 \\
\end{array}$ & $a b$ & $\begin{array}{l}\text { Granola } \\
0,33 \\
1,28 \\
\end{array}$ & a $a$ & $\begin{array}{l}\text { Lolita } \\
0,14\end{array}$ & a $a$ & $\begin{array}{l}\text { Marabel } \\
0,41 \\
0,81 \\
\end{array}$ & a $b$ & $\begin{array}{l}\text { Nicola } \\
0,65 \\
1,65 \\
\end{array}$ & a $b$ & $\begin{array}{l}\text { Renate } \\
0,29 \\
18,38 \\
\end{array}$ & a $a$ \\
\hline & 8 Monate Lagerung & B & $\begin{array}{ll}\text { Mark a } & \\
\text { Schale } & \end{array}$ & $\begin{array}{l}\text { Adretta } \\
0,26 \\
0,99\end{array}$ & a $a$ & $\begin{array}{l}\text { Afra } \\
0,40 \\
1,46 \\
\end{array}$ & $\mathbf{a} b$ & $\begin{array}{l}\text { Gala } \\
0,58 \\
0,38 \\
\end{array}$ & $a b$ & $\begin{array}{l}\text { Granola } \\
0,26 \\
0,47\end{array}$ & a $a$ & $\begin{array}{l}\text { Lolita } \\
1,23 \\
0,75 \\
\end{array}$ & a $a$ & $\begin{array}{l}\text { Marabel } \\
0,32 \\
0,39\end{array}$ & a $b$ & $\begin{array}{l}\text { Nicola } \\
1,16 \\
0,88 \\
\end{array}$ & a $b$ & $\begin{array}{l}\text { Renate } \\
0,45 \\
0,50 \\
\end{array}$ & a $a$ \\
\hline
\end{tabular}


Tabelle 10.59 Grad der Pektinveresterung [Mittelwert $(\bar{x})$ und Standartabweichung $\left(\sigma_{\mathrm{x}}\right)$ ] $(\%)$ in Sorten und Knollen verschiedener spezifischer Dichten $\left(\mathrm{kg} \mathrm{L}^{-1}\right)$ nach der Ernte 2005 und nach fünf und acht Monaten Lagerung

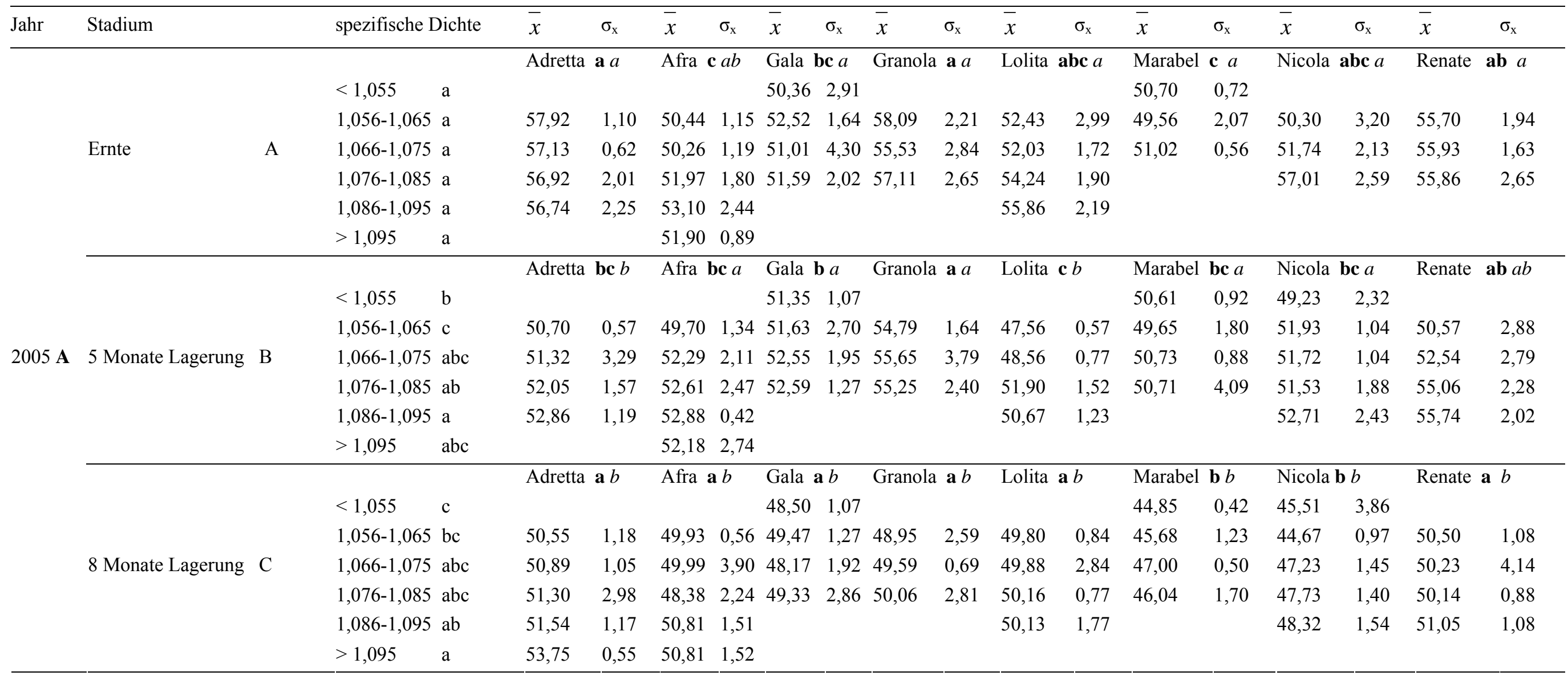


Tabelle 10.60 Grad der Pektinveresterung [Einzelwerte] (\%) in Sorten und Knollen verschiedener spezifischer Dichten (kg L $\left.\mathrm{L}^{-1}\right)$ nach der Ernte 2006 und nach fünf und acht Monaten Lagerung

\begin{tabular}{|c|c|c|c|c|c|c|c|c|c|c|c|c|}
\hline Jahr & \multicolumn{2}{|l|}{ Stadium } & \multicolumn{2}{|c|}{ spezifische Dichte } & \multicolumn{8}{|c|}{ Sorten } \\
\hline & & & $<1,055$ & $\mathrm{a}$ & Adretta $\mathbf{b} b$ & Afra abc $b$ & $\begin{array}{l}\text { Gala abc } a \\
85,12\end{array}$ & Granola c $b$ & Lolita $\mathbf{b} b$ & $\begin{array}{l}\text { Marabel abc } b \\
70,76\end{array}$ & Nicola $\mathbf{a b c} b$ & Renate $\mathbf{a b} b$ \\
\hline & & & $1,056-1,065$ & $\mathrm{a}$ & 40,34 & & 41,37 & 3,44 & 42,85 & 38,79 & 42,81 & 75,57 \\
\hline & Ernte & B & $1,066-1,075$ & $\mathrm{a}$ & 38,47 & 23,77 & 76,73 & 4,19 & 36,43 & 14,02 & 47,99 & 46,51 \\
\hline & & & $1,076-1,085$ & $\mathrm{a}$ & 39,51 & 29,01 & & 36,05 & 7,68 & 26,33 & 43,84 & 49,05 \\
\hline & & & $1,086-1,095$ & $\mathrm{a}$ & 11,39 & 45,50 & & & & & & \\
\hline & & & $>1,095$ & $\mathrm{a}$ & 27,01 & & & & & & & \\
\hline & & & $<1,055$ & $\mathrm{a}$ & Adretta $\mathbf{a b} a$ & Afra $\mathbf{a b} a$ & $\begin{array}{l}\text { Gala ab } a \\
76,73\end{array}$ & Granola b $a$ & Lolita ab $a$ & Marabel ab $a$ & Nicola ab $a$ & Renate a $a$ \\
\hline & & & $1,056-1,065$ & $\mathrm{a}$ & 59,15 & & 68,26 & 62,49 & 95,33 & 81,94 & 67,01 & 86,70 \\
\hline \multirow[t]{10}{*}{2006 A } & 5 Monate Lagerung & A & $1,066-1,075$ & $\mathrm{a}$ & 58,18 & 67,67 & 77,09 & 42,60 & 58,98 & 62,46 & 67,97 & 122,68 \\
\hline & & & $1,076-1,085$ & $\mathrm{a}$ & 75,93 & 74,27 & 97,72 & 50,72 & 66,10 & 68,86 & 56,86 & 79,99 \\
\hline & & & $1,086-1,095$ & $\mathrm{a}$ & 56,39 & 83,77 & & & 64,60 & & 113,73 & 78,67 \\
\hline & & & $>1,095$ & $\mathrm{a}$ & 70,66 & 87,13 & & & & & & \\
\hline & & & $<1,055$ & $\mathrm{a}$ & Adretta $\mathbf{a} b$ & Afra $\mathbf{a} b$ & $\begin{array}{l}\text { Gala a } b \\
42,66\end{array}$ & Granola a $b$ & Lolita $\mathbf{a} b$ & Marabel a $b$ & Nicola $\mathbf{a} b$ & Renate a $c$ \\
\hline & & & $1,056-1,065$ & $\mathrm{a}$ & & & 38,58 & 13,63 & 43,80 & 12,87 & 43,65 & 36,31 \\
\hline & 8 Monate Lagerung & $\mathrm{C}$ & $1,066-1,075$ & $\mathrm{a}$ & 17,24 & 29,76 & 46,97 & 8,08 & 36,48 & 39,42 & 22,61 & 34,12 \\
\hline & & & $1,075-1,085$ & $5 \mathrm{a}$ & 11,13 & 20,16 & 26,54 & 19,75 & 20,66 & 21,63 & 16,10 & 20,74 \\
\hline & & & $1,085-1,095$ & $5 \mathrm{a}$ & 28,50 & 9,67 & & 17,17 & 9,63 & 24,04 & 23,69 & 33,42 \\
\hline & & & $>1,095$ & $\mathrm{a}$ & 12,45 & 24,32 & & & & & & 20,34 \\
\hline
\end{tabular}


Tabelle 10.61 Grad der Pektinveresterung [Einzelwerte] (\%) in Sorten und Knollen verschiedener spezifischer Dichten (kg L $\left.\mathrm{L}^{-1}\right)$ nach der Ernte 2007 und nach fünf und acht Monaten Lagerung

\begin{tabular}{|c|c|c|c|c|c|c|c|c|c|c|c|c|}
\hline Jahr & Stadium & & spezifische D & ichte & & & & & Sorten & & & \\
\hline & & & $<1,055$ & $\mathrm{a}$ & Adretta a $a$ & Afra a $a$ & $\begin{array}{l}\text { Gala a } b \\
7,37\end{array}$ & Granola a $a$ & Lolita a $a$ & Marabel a $a$ & Nicola a $a$ & Renate a $a$ \\
\hline & & & $1,056-1,065$ & $\mathrm{a}$ & 42,88 & & 5,83 & 22,98 & 11,78 & 10,29 & 17,57 & 49,32 \\
\hline & Ernte & $\mathrm{B}$ & $1,066-1,075$ & $\mathrm{a}$ & 38,99 & 47,06 & 19,61 & 40,61 & 57,32 & 54,69 & 17,76 & 58,04 \\
\hline & & & $1,076-1,085$ & $\mathrm{a}$ & 35,95 & 37,61 & 15,13 & 37,84 & 59,71 & 3,46 & 24,95 & 59,50 \\
\hline & & & $1,086-1,095$ & $\mathrm{a}$ & 39,89 & 48,15 & & & 42,71 & 64,85 & & \\
\hline & & & $>1,095$ & $\mathrm{a}$ & & & & & & & & \\
\hline & & & $<1,055$ & $\mathrm{a}$ & Adretta a $a$ & Afra a $a$ & $\begin{array}{l}\text { Gala a } a \\
52,99\end{array}$ & Granola a $a$ & Lolita a $a$ & Marabel a $a$ & Nicola a $a$ & Renate a $a$ \\
\hline & & & $1,056-1,065$ & $\mathrm{a}$ & 32,04 & & 64,23 & 49,43 & 33,31 & & 44,60 & 51,67 \\
\hline 2007 B & 5 Monate Lagerung & A & $1,066-1,075$ & $\mathrm{a}$ & 59,07 & 75,90 & 43,01 & 40,17 & 48,99 & 26,53 & 31,97 & 45,23 \\
\hline & & & $1,076-1,085$ & $\mathrm{a}$ & 46,93 & 49,85 & & 40,28 & 21,15 & 3,79 & 9,84 & 24,44 \\
\hline & & & $1,086-1,095$ & $\mathrm{a}$ & 8,71 & 17,50 & & 80,64 & 47,72 & 33,70 & & 33,59 \\
\hline & & & $>1,095$ & $\mathrm{a}$ & 48,42 & 27,48 & & & 46,86 & & & \\
\hline & & & $<1,055$ & $\mathrm{a}$ & Adretta $\mathbf{a b} a$ & Afra a $a$ & $\begin{array}{l}\text { Gala ab } b \\
33,81\end{array}$ & $\begin{array}{l}\text { Granola ab } a \\
48,43\end{array}$ & Lolita ab $a$ & Marabel b $a$ & Nicola ab $a$ & Renate $\mathbf{a b} a$ \\
\hline & & & $1,056-1,065$ & $a b$ & 37,42 & 49,44 & 20,53 & 15,58 & 35,20 & 9,00 & 38,20 & 25,30 \\
\hline & 8 Monate Lagerung & B & $1,066-1,075$ & $a b$ & & 37,95 & 26,66 & 24,14 & 20,12 & 0,89 & 1,64 & 11,99 \\
\hline & & & $1,076-1,085$ & $a b$ & 31,45 & 43,99 & 22,45 & 25,63 & 20,32 & 28,59 & 13,23 & 34,47 \\
\hline & & & $1,086-1,095$ & $a b$ & 38,31 & 36,10 & & & 25,64 & & 21,21 & 31,68 \\
\hline & & & $>1,095$ & $\mathrm{~b}$ & 22,74 & 13,93 & & & & & & \\
\hline
\end{tabular}




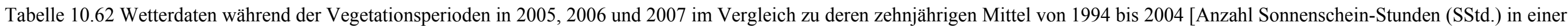

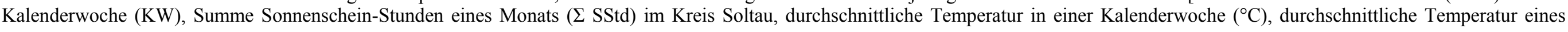

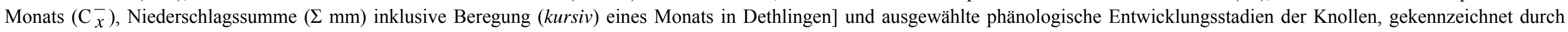
eine zweistellige BBCH-Codierung (Meier 2001) in denen u. a. die Krautabtötung (BBCH 48) und Ernte der Knollen (BBCH 99) als Vorernte (VE) und Ernte (HE) erfolgte

\begin{tabular}{|c|c|c|c|c|c|c|c|c|c|c|c|c|c|c|c|c|c|c|c|c|c|c|}
\hline \multicolumn{2}{|c|}{ Vegetationsperiode } & \multicolumn{6}{|c|}{2005} & \multicolumn{6}{|c|}{2006} & \multicolumn{6}{|c|}{2007} & \multicolumn{3}{|c|}{$1994-2004 \bar{x}$} \\
\hline $\mathrm{KW}$ & Monat & $\mathrm{BBCH}$ & SStd. & $\Sigma$ SStd. & ${ }^{\circ} \mathrm{C}$ & ${ }^{\circ} \mathrm{C} \bar{x}$ & $\Sigma \mathrm{mm}$ & $\mathrm{BBCH}$ & SStd. & $\Sigma$ SStd. & ${ }^{\circ} \mathrm{C}$ & $\mathrm{C} \bar{x}$ & $\Sigma \mathrm{mm}$ & $\mathrm{BBCH}$ & SStd. & $\Sigma$ SStd. & ${ }^{\circ} \mathrm{C}$ & ${ }^{\circ} \mathrm{C} \bar{x}$ & $\Sigma \mathrm{mm}$ & $\Sigma$ SStd. & ${ }^{\circ} \mathrm{C} \bar{x}$ & $\Sigma \mathrm{mm}$ \\
\hline 13 & März & & & & & & & & & & & & 77,0 & 0 & 76,6 & 76,6 & 9,7 & 9,7 & 92,5 & 108,9 & 4,4 & 65,8 \\
\hline 14 & April & 0 & 31,2 & & 7,6 & & 51,0 & & 25,9 & & 4,8 & & & & 37,1 & & 7,8 & & & & & \\
\hline 15 & April & & 40,5 & & 11,9 & & & & 15,7 & & 6,4 & & & & 51,7 & & 13,3 & & & & & \\
\hline 16 & April & 9 & 58,4 & & 7,3 & & & 0 & 19,5 & & 9,7 & & & 9 & 56,4 & & 9,1 & & 7,5 & & & \\
\hline 17 & April & & 37,4 & 167,5 & 12,5 & 9,8 & 36,5 & 9 & 39,6 & 100,7 & 10,3 & 7,8 & 84,0 & 10 & 74,4 & 296,1 & 15,7 & 11,5 & 22,5 & 159,3 & 8,5 & 51,3 \\
\hline 18 & Mai & & 31,8 & & 10,7 & & & 10 & 76,1 & & 15,8 & & & & 90,5 & & 13,7 & & & & & \\
\hline 19 & Mai & & 33,8 & & 8,9 & & & & 76,7 & & 15,2 & & & & 22,6 & & 12,3 & & & & & \\
\hline 20 & Mai & 40 & 39,9 & & 12,1 & & & 40 & 28,8 & & 13,6 & & & 40 & 50,9 & & 13,1 & & 104,5 & & & \\
\hline 21 & Mai & & 57,9 & 163,4 & 19,0 & 12,7 & 108,0 & & 24,3 & 205,9 & 11,4 & 14,0 & 91,0 & & 49,3 & 213,3 & 18,7 & 14,5 & & 199,6 & 13,0 & 58,5 \\
\hline 22 & Juni & & 25,6 & & 13,1 & & & & 27,2 & & 10,3 & & & & 32,0 & & 14,9 & & & & & \\
\hline 23 & Juni & 41 & 44,6 & & 11,5 & & & 41 & 79,1 & & 15,8 & & & 41 & 61,4 & & 21,8 & & & & & \\
\hline 24 & Juni & & 74,2 & & 17,2 & & & & 61,7 & & 19,2 & & & & 50,2 & & 19,7 & & & & & \\
\hline 25 & Juni & & 76,5 & 220,9 & 19,7 & 15,4 & & & 49,7 & 217,7 & 19,3 & 16,1 & & & 34,0 & 177,7 & 17,8 & 18,6 & 55,0 & 196,1 & 15,6 & 66,3 \\
\hline 26 & Juli & & 44,8 & & 17,9 & & 53,0 & & 65,6 & & 18,1 & & 47,0 & & 24,5 & & 15,1 & & 30,0 & & & \\
\hline 27 & Juli & & 38,7 & & 17,8 & & 90,0 & & 74,9 & & 23,0 & & 60,0 & 48 (VE) & 31,9 & & 15,4 & & & & & \\
\hline 28 & Juli & & 74,1 & & 20,1 & & & & 84,1 & & 20,1 & & & & 37,6 & & 17,6 & & & & & \\
\hline 29 & Juli & & 22,6 & & 16,4 & & & 48 (VE) & 89,9 & & 24,1 & & & & 65,6 & & 20,3 & & 115,5 & & & \\
\hline 30 & Juli & & 34,3 & 214,5 & 18,8 & 18,2 & 103,0 & & 57,6 & 372,1 & 24,2 & 21,9 & 19,0 & & 25,6 & 185,2 & 16,3 & 16,9 & & 210,2 & 17,9 & 82,7 \\
\hline 31 & August & & 26,4 & & 14,3 & & 30,0 & 48 (HE) & 38,4 & & 17,7 & & 90,0 & 48 (HE) & 55,4 & & 15,9 & & & & & \\
\hline 32 & August & 48 (HE) & 8,2 & & 14,4 & & & & 38,4 & & 16,6 & & & & 39,8 & & 19,3 & & & & & \\
\hline 33 & August & & 36,3 & & 18,5 & & & & 29,7 & & 16,9 & & & & 44,2 & & 17,2 & & & & & \\
\hline 34 & August & & 31,2 & 102,1 & 15,5 & 15,7 & & & 21,1 & 127,6 & 15,9 & 16,8 & & & 30,2 & 169,5 & 17,8 & 17,6 & 68,5 & 198,4 & 18,2 & 63,9 \\
\hline 35 & September & & 65,8 & & 18,4 & & 73,0 & & 31,5 & & 16,2 & & 117,0 & 99 (VE) & 18,6 & & 13,4 & & & & & \\
\hline 36 & September & 99 (HE) & 63,3 & & 19,7 & & & & 40,9 & & 15,8 & & & & 38,2 & & 13,9 & & & & & \\
\hline 37 & September & & 47,6 & & 16,6 & & & 99 (VE) & 78,4 & & 19,1 & & & 99 (HE) & 34,2 & & 13,1 & & & & & \\
\hline 38 & September & & 66,0 & & 13,3 & & & & 56,4 & & 16,4 & & & & 32,5 & 123,4 & 13,3 & 13,4 & 31,5 & 151,0 & 13,5 & 75,6 \\
\hline 39 & September & & 22,9 & 265,6 & 11,0 & 15,8 & 19,0 & 99 (HE) & 31,2 & 238,4 & 16,0 & 16,7 & 15,0 & & & & & & & & & \\
\hline$\overline{\Sigma S S t c}$ & & & & 1134,0 & & & & & & 1262,4 & & & & & & 1241,7 & & & & 1223,5 & & \\
\hline${ }^{\circ} \mathrm{C} \bar{x}$ & & & & & & 14,6 & & & & & & 15,5 & & & & & & 14,6 & & & 13,0 & \\
\hline$\Sigma \mathrm{mm}$ & & & & & & & 563,5 & & & & & & 600,0 & & & & & & 527,5 & & & 464,1 \\
\hline
\end{tabular}


Tabelle 10.63 Ausgewählte Eigenschaften der untersuchten Sorten (Bundessortenamt 2004, 2005, 2006)

\begin{tabular}{llll}
\hline Sorte & Reifegruppe & Stärkegehalt & Neigung zu Schwarzfleckigkeit \\
\hline Afra & mittelfrüh & mittel bis hoch & mittel \\
Adretta & mittelfrüh & mittel & mittel \\
Granola & mittelfrüh & niedrig bis mittel & mittel \\
Nicola & mittelfrüh & niedrig bis mittel & niedrig \\
Renate & früh & niedrig bis mittel & niedrig bis mittel \\
Gala & früh & niedrig & sehr niedrig \\
Marabel & früh & niedrig & sehr niedrig \\
Lolita & mittelfrüh & niedrig bis mittel & sehr niedrig \\
\hline
\end{tabular}


Tabelle 10.64 Standorteigenschaften und Informationen zum Anbau der Kartoffeln

\begin{tabular}{|c|c|c|c|c|c|c|}
\hline Anbauinformation & \multicolumn{6}{|c|}{ Versuchsjahr } \\
\hline & \multicolumn{2}{|c|}{2005} & \multicolumn{2}{|c|}{2006} & \multicolumn{2}{|c|}{2007} \\
\hline Versuchsstandort & \multicolumn{6}{|c|}{ Dethlingen } \\
\hline \multicolumn{7}{|c|}{ Eigenschaften des Versuchsstandortes vor jeder Vegetationsperiode (Gehaltsklasse) } \\
\hline Bodenart & \multicolumn{6}{|c|}{ humoser Sand } \\
\hline Ackerzahl & \multicolumn{6}{|l|}{25} \\
\hline Tiefe $(\mathrm{cm})$ & 0 bis 30 & 30 bis 60 & 0 bis 30 & 30 bis 60 & 0 bis 30 & 30 bis 60 \\
\hline $\mathrm{pH}-\mathrm{Wert}\left(\mathrm{CaCl}_{2}\right)$ & 5,2 & 5,0 & 5,6 & 5,7 & 5,3 & 5,1 \\
\hline Wassergehalt $\left(\mathrm{g} \mathrm{kg}^{-1}\right)$ & 160 & 70 & 160 & 100 & 140 & 60 \\
\hline$P\left(\mathrm{~g} \mathrm{~kg}^{-1}\right)$ & 57 (B) & $2(\mathrm{~A})$ & $58(\mathrm{C})$ & 4 (A) & $44(\mathrm{~B})$ & $2(\mathrm{~A})$ \\
\hline $\mathrm{K}\left(\mathrm{g} \mathrm{kg}^{-1}\right)$ & 60 (B) & $40(\mathrm{~B})$ & $67(\mathrm{C})$ & $46(\mathrm{C})$ & $55(\mathrm{C})$ & $23(\mathrm{~A})$ \\
\hline $\operatorname{Mg}\left(\mathrm{g} \mathrm{kg}^{-1}\right)$ & $72(\mathrm{C})$ & $13(\mathrm{~A})$ & $42(\mathrm{C})$ & $12(\mathrm{~A})$ & $57(\mathrm{C})$ & 7 (A) \\
\hline $\mathrm{B}\left(\mathrm{mg} \mathrm{kg}^{-1}\right)$ & $0,6(\mathrm{C})$ & $0,4(\mathrm{C})$ & $0,3(\mathrm{C})$ & $0,1(\mathrm{~A})$ & $0,2(\mathrm{C})$ & $0,04(\mathrm{~A})$ \\
\hline $\mathrm{Cu}\left(\mathrm{mg} \mathrm{kg}^{-1}\right)$ & $3,7(\mathrm{E})$ & $0,2(\mathrm{~A})$ & $3,9(\mathrm{E})$ & $0,3(\mathrm{~A})$ & $1,1(\mathrm{C})$ & $0,1(\mathrm{~A})$ \\
\hline $\operatorname{Mn}\left(\mathrm{mg} \mathrm{kg}^{-1}\right)$ & $5,1(\mathrm{~A})$ & $0,4(\mathrm{~A})$ & $16,1(\mathrm{~A})$ & $1,1(\mathrm{~A})$ & $2,8(\mathrm{~A})$ & $0,3(\mathrm{~A})$ \\
\hline $\mathrm{NH}_{4}\left(\mathrm{~kg} \mathrm{ha}^{-1}\right)$ & 12 & 0 & 4 & 2 & 5 & 2 \\
\hline $\mathrm{NO}_{3}\left(\mathrm{~kg} \mathrm{ha}^{-1}\right)$ & - & - & 5 & 3 & 10 & 4 \\
\hline $\mathrm{N}_{\min }\left(\mathrm{kg} \mathrm{ha}^{-1}\right)$ & 12 & 7 & 9 & 5 & 15 & 6 \\
\hline \multicolumn{7}{|l|}{ Letzte Vorfrucht } \\
\hline Legen & \multicolumn{2}{|c|}{ 05. April } & \multicolumn{2}{|c|}{ 15.April } & \multicolumn{2}{|c|}{ 05. April } \\
\hline \multicolumn{7}{|l|}{ Düngung $\left(\mathrm{kg} \mathrm{ha}^{-1}\right)$} \\
\hline $\mathrm{N}$ & \multicolumn{2}{|c|}{120} & \multicolumn{2}{|c|}{119} & \multicolumn{2}{|c|}{117} \\
\hline $\mathrm{P}_{2} \mathrm{O}_{5}$ & \multicolumn{2}{|c|}{108} & \multicolumn{2}{|c|}{92} & \multicolumn{2}{|c|}{120} \\
\hline $\mathrm{K}_{2} \mathrm{O}$ & \multicolumn{2}{|c|}{280} & \multicolumn{2}{|c|}{240} & \multicolumn{2}{|c|}{240} \\
\hline $\mathrm{CaO}$ & \multicolumn{2}{|c|}{ - } & \multicolumn{2}{|c|}{-} & \multicolumn{2}{|c|}{58} \\
\hline \multicolumn{7}{|c|}{ Pflanzenschutz $\left(\mathrm{L} \mathrm{ha}^{-1}\right)$ oder $\left(\mathrm{kg} \mathrm{ha}^{-1}\right)$} \\
\hline Vorernte & \multicolumn{2}{|c|}{-} & \multicolumn{2}{|c|}{ 11. bis 15 . Sept. } & \multicolumn{2}{|c|}{ 27. bis 30 . August } \\
\hline Ernte & \multicolumn{2}{|c|}{ 05. bis 08. Sept. } & \multicolumn{2}{|c|}{ 25. bis 29. Sept. } & \multicolumn{2}{|c|}{ 11. bis 15 . Sept. } \\
\hline
\end{tabular}




\section{Danksagung}

An dieser Stelle möchte ich mich herzlich bei Prof. Dr. E. Pawelzik bedanken. Sie hatte einen großen Anteil an der Verwirklichung des Forschungsvorhabens und gab mir die Möglichkeit, wissenschaftlich zu arbeiten. An ihre fachliche Anleitung und ihre wissenschaftliche Beratung und Betreuung werde ich mich stets gern errinnern.

Ich bedanke mich ebenfalls bei Prof. Dr. B. Märländer und PD Dr. J. Schulze für die wissenschaftliche Diskussion und Bewertung dieser Arbeit.

Außerdem danke ich Prof. Dr. W. Römer, Prof. Dr. N. Claassen, Prof. Dr. E. Przemeck, PD Dr. A. Keutgen, PD Dr. J. Niemeyer, Dr. B. Steingrobe, Dr. I. Smit, Dr. R. Peters, Dr. N. Haase, Dr. W. B. Herppich und Dr. T. Hoffmann für ihre stete Bereitschaft zum intensiven wissenschaftlichen Meinungsaustausch.

Für die umfangreiche fachliche Unterstützung und das außerordentliche Engagement bei der Bearbeitung der einzelnen Aufgabengebiete bedanke ich mich bei C. Conradt, B. Egger, B. Eichenberg, M. Grabmüller, M. Hanke, U. Hill, R. Hilmer, G. Jansen, J. Kobbe, S. Koch, J. Krome, M. Niebuhr, M. Noltkämper, B. Wulf und ganz besonders bei E. Krüger.

Ein besonderes Dankeschön gilt meiner Familie, die mich in meiner Arbeit fortwährend unterstützt hat und stets Verständnis zeigte.

Für die finanzielle Förderung bedanke ich mich bei dem Bundesministerium für Ernährung, Landwirtschaft und Verbraucherschutz, vertreten durch die Bundesanstalt für Landwirtschaft und Ernährung und bei der Gemeinschaft zur Förderung der privaten deutschen Pflanzenzüchtung. 


\section{Lebenslauf}

\section{Persönliche Daten}

Name: $\quad$ Antje Wulkow

Geburtstag/-ort: $\quad$ 17.12.1979 in Schwedt/Oder

\section{Ausbildung}

07.2005

Abschluss des Studiums als M.Sc. der Agrarwissenschaften an der Georg-August-Universität Göttingen, Studienrichtung Pflanzenproduktion

06.1999 Abschluss des Abiturs am Albert-Einstein-Gymnasium Angermünde

\section{Berufspraxis}

seit 08.2005

wissenschaftliche Mitarbeiterin am Department für Nutzpflanzenwissenschaften, Abteilung Qualität pflanzlicher Erzeugnisse der Georg-August-Universität Göttingen

08.2007-07.2008 wissenschaftliche Hilfskraft am Institut für Tierphysiology und Tierernährung der Georg-August-Universität Göttingen

03.2000-07.2005 studentische Hilfskraft am Department für Nutzpflanzenwissenschaften, Abteilung Qualität pflanzlicher Erzeugnisse und Abteilung Rhizosphäre und in der Verwaltung der Georg-August-Universität Göttingen

06.2001-10.2001 Praktikum auf dem Landwirtschaftsbetrieb der Familie Buritz in Bad Harzburg

06.2000-10.2000 Praktikum am Zentrum für angewandte Landnutzungsforschung (ZALF), Standort Prenzlau 